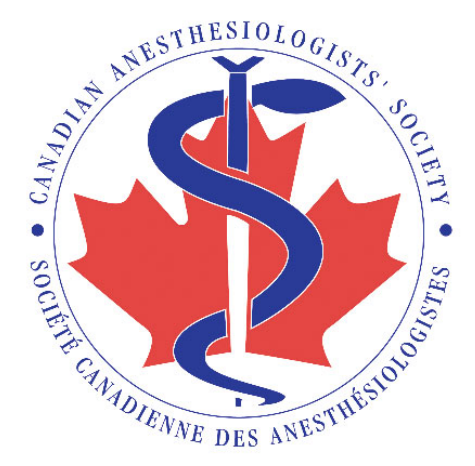

\title{
2020 CAS VIRTUAL ANNUAL MEETING ABSTRACTS
}

ABSTRACTS ARE ARRANGED BY LEARNING TRACK AS FOLLOWS:






\section{AIRWAY MANAGEMENT}

\section{A Study Comparing Lightwand and Glidescope in Difficult Tracheal Intubation Involving Cadavers with a Cervical Spine Collar}

Ana Hung ${ }^{1}$; Haotian Wang ${ }^{2}$; Christopher Hung ${ }^{1}$; Matthew d'Entremont ${ }^{2}$; Carrie Goodine ${ }^{2}$; Gaurav Arora²; Jasmin Astle²; Irene Sadek ${ }^{3}$; Orlando Hung ${ }^{2}$; Andrew Milne ${ }^{2}$

1 Dalhousie Medical School, Halifax, Canada

2 Department of Anesthesia, Pain Management and Perioperative Medicine, Dalhousie University, Halifax, Canada

3 Department of Pathology, Dalhousie University, Halifax, Canada

Introduction: Restricted mouth opening can result in difficulty with tracheal intubation by direct laryngoscopy and the inability to secure an airway with tracheal intubation when needed is associated with significant morbidity and mortality. The American society of anesthesiologists (ASA) practice guidelines recommend video laryngoscopes and the Lightwand (LW) as alternative intubation devices as neither of them requires direct visualization of the glottis.

Objectives: The goal of this pilot study was to compare the performance of Glidescope $(\mathrm{GS})$ and the new prototype LW device in a simulated restricted mouth opening with cervical spine collars in cadavers.

Methods: Research Ethics Board approval was obtained from the local REB (Nova Scotia Health Authority, Halifax, Nova Scotia, Canada) and four clinical grade cadavers were obtained for this study. Six staff anesthesiologists were recruited and watched an instructional video on the prototype LW device prior to being randomized to one of four cadavers with cervical collars in place. They were asked to perform tracheal intubation with each intubation device while being recorded on video. All videos were reviewed offline by an independent investigator to determine the time-to-intubation (TTI), the number of attempts, the time to airway management (TAM) or successful device insertion into the trachea and the success rate of tracheal intubation. After each intubation, the participant used a visual analog scale (VAS) to report the subjective degree of difficult intubation using the intubation device.

Results: Overall, there were no significant differences between the LW and the GS across all outcomes. Linear Mixed Models were used to analyze the data. There was no effect of device on TTI $(95 \% \mathrm{Cl}[-13.4,16.7], p=0.841)$ and TAM $(95 \% \mathrm{Cl}[-13.8,15], p=0.933)$. In terms of self reported VAS scores, there was no different between devices $(95 \% \mathrm{Cl}[-10.8,13], \mathrm{p}=0.859)$.

Conclusion: The results of our study suggest GS and LW are similarly effective in intubating cadavers with a cervical spine collar. LW relies on transillumination of the anterior neck soft tissue and is a significantly smaller device designed to be malleable, allowing for adjustments such as the degree of tip deflection. While these features might make the LW a better device to use in patients with a small mouth opening as compared to a video laryngoscope, a large clinical study is necessary to determine its utility in patients with limited mouth opening. 


\section{REFERENCES:}

Apfelbaum JL, Hagberg CA, Caplan RA et al. Anesthesiology. 2013; 118: 251-270. 


\section{Awake Videolaryngoscopy in the Management of Acute Angioedema}

Luke Anderson $^{1,2}$; Gary Leung ${ }^{1}$; Kelvin Kong ${ }^{2,3,4}$; Joanne Walsh ${ }^{1}$

1 Department of Anaesthesia and Pain Medicine, John Hunter Hospital, Newcastle, New South Wales, Australia

2 University of Newcastle, Newcastle, New South Wales, Australia

3 Department of Otolaryngology, John Hunter Hospital and John Hunter Children's Hospital, Newcastle, New South Wales, Australia

4 University of New South Wales, Sydney, New South Wales, Australia

Introduction: Awake videolaryngoscopy has emerged as a viable alternative to awake fibreoptic intubation in the management of the anticipated difficult airway. In the elective setting, awake videolaryngoscopy is associated with shorter time to intubation compared to awake fibreoptic intubation, without any significant difference in failure rate, patient satisfaction or incidence of adverse events (1). However, little evidence exists to guide the use of awake videolaryngoscopy in the emergency setting.

We describe a case of successful tracheal intubation using awake videolaryngoscopy in a patient presenting with acute angioedema.

Case Presentation: Patient consent was obtained for publication of this case.

A 75-year-old woman presented to a regional emergency department with tongue swelling, drooling, periorbital oedema and stridor shortly after consuming prawns. She had a background history of hypertension, treated with an ACE inhibitor. She was treated with nebulised adrenaline, hydrocortisone and promethazine before being transferred to a tertiary hospital for emergent airway management. Fibreoptic nasoendoscopy revealed significant supraglottic swelling.

A nasal awake fibreoptic intubation was planned initially. Glycopyrrolate premedication was given. The nares were topicalised with Co-Phenylcaine Forte (5\% lignocaine with $0.5 \%$ phenylephrine) and a 6.0 nasopharyngeal airway inserted. The nasopharynx and oropharynx were topicalised with $4 \%$ lignocaine using a mucosal atomiser device and DeVilbiss atomiser. Optiflow Transnasal Humidified Rapid-Insufflation Ventilatory Exchange (THRIVE) was applied and titrated to patient comfort.

The airway plan was changed when mouth opening sufficient to allow insertion of a laryngoscope blade was noted. A McGrath videolaryngoscope with Mac 4 blade was inserted while in the semi-recumbent position. A grade $2 b$ view of the glottis was visualised on videolaryngoscopy. A grade 4 direct laryngoscopic view was noted at the time. The cords were sprayed with $4 \%$ lignocaine and a gum elastic bougie inserted to facilitate passage of a size 7.0 endotracheal tube. After confirmation of tracheal tube placement, general anaesthesia was induced.

The procedure was well tolerated by the patient, and no sedation was required.

The patient was extubated 8 days later. Testing confirmed ACE inhibitor induced angioedema. 
Discussion: We present the first case report of awake videolaryngoscopy in the management acute angioedema. Awake videolaryngoscopy has several potential advantages over fibreoptic intubation including a wider view of the airway, no limitation of endotracheal tube diameter, and the ability to use adjuncts such as the gum elastic bougie. Videolaryngoscopy is an easier skill to learn and maintain (2).

Our case highlights the value of videolaryngoscopy as an alternative to fibreoptic intubation in carefully selected patients in the emergency setting.

\section{REFERENCES:}

1. Alhomary M, Ramadan E, Curran E, Walsh S. Videolaryngoscopy vs. fibreoptic bronchoscopy for awake tracheal intubation: a systematic review and meta-analysis. Anaesthesia. 2018;73(9):1151-1161.

2. Fitzgerald E, Hodzovic I, Smith AD. 'From darkness into light': time to make awake intubation with videolaryngoscopy the primary technique for an anticipated difficult airway? Anaesthesia 2015;70(4):387-92. 


\section{Canadian Emergency Physician Attitudes Toward Endotracheal Intubation for Aspiration} Prophylaxis

M. Brendan Munn ${ }^{1}$; J. Ferdows Laraya ${ }^{2}$; Gabrielle Boivin Arcouette $^{3}$; Etienne van der Linde ${ }^{4}$; Sheila Turris ${ }^{5}$; Adam Lund ${ }^{5}$

1 Department of Family Medicine, University of British Columbia, Vancouver, Canada

2 Department of Anesthesia, McGill University, Montreal, Canada

3 Family Medicine Residency Program, University of Montreal, Montreal, Canada

4 Disciplines of Emergency and Family Medicine, Memorial University, St John's, Canada

5 Department of Emergency Medicine, University of British Columbia, Vancouver, Canada

Introduction: Emergency patients with decreased level of consciousness often undergo intubation purely for airway protection from aspiration. However, the true risk of aspiration is unclear and intubation poses risks. Anecdotally, experienced emergency physicians often defer intubation in these patients while others intubate to decrease the perceived clinical and medicolegal consequences. No literature exists on the intubation practices of emergency physicians in these cases.

Methods: Ethics approval was obtained from the local REB. An online questionnaire was circulated to members of the Canadian Association of Emergency Physicians. Participants were asked questions regarding two common clinical cases with decreased level of consciousness: (1) acute, uncomplicated alcohol intoxication and (2) acute, uncomplicated seizure. For each case, providers' perceptions of aspiration risk, the standard of care, and the need for intubation were assessed.

Results: 128 of the 1546 Canadian physicians contacted (8.3\%) provided responses. Respondents had a median of 15 years of experience, $88 \%$ had CCFP-EM or FRCPC certification, and most worked in urban centers. When intubating, $98 \%$ agreed they were competent and $90 \%$ agreed they were well supported. A minority $(17.4 \%)$ considered GCS $<8$ an independent indication for intubation. For the alcohol intoxication case, $88 \%$ agreed that aspiration risk was present but only $11 \%$ agreed they commonly intubate. Only $17 \%$ agreed intubation was standard care, and only $0.8 \%$ felt their colleagues always intubate such patients. For the seizure case, $65 \%$ agreed aspiration risk existed but only $3 \%$ agreed they commonly intubate, $1 \%$ felt colleagues always intubated, and $5 \%$ agreed intubation was standard of care. Additional factors felt to compel intubation (394 total) and support non-intubation (366 total) were compiled and categorized; the most common themes emerging were objective evidence of emesis or aspiration, other standard indications for intubation, head trauma, co-ingestions, comorbidities and clinical instability.

Conclusion: It is acceptable and standard practice to avoid intubating a select subset of intoxicated and post-seizure emergency department patients despite aspiration risk. Most physicians do not view the dogma of "GCS 8, intubate" as an absolute indication for intubation in these patients. Future research is aimed at identifying key factors and evidence supporting intubation for the prevention of aspiration, as well as the development of a validated clinical decision rule for common emergency presentations. 


\section{Comparing a Prototype Lightwand vs Glidescope for Tracheal Intubation in Soiled} Cadaver Airways

Christopher Hung ${ }^{1,2}$; Haotian Wang ${ }^{2}$; Ana Hung ${ }^{1}$; Matthew d'Entremont ${ }^{2}$; Carrie Goodine ${ }^{2}$, Gaurav Arora ${ }^{2}$; Jasmin Astle; ${ }^{2}$;rene Sadek ${ }^{3}$; Orlando Hung ${ }^{2}$; Andrew Milne ${ }^{2}$

1 Dalhousie Medical School, Halifax, Nova Scotia, Canada

2 Department of Anesthesia, Pain Management and Perioperative Medicine, Dalhousie University, Halifax, Nova Scotia, Canada

3 Department of Pathology, Dalhousie University, Halifax, Nova Scotia, Canada

Introduction: Soiled airways have been negatively associated with endotracheal intubation success rates by direct or videolaryngoscopy. ${ }^{1}$ Transtracheal illumination techniques are a proposed alternative as they do not require visualization of the glottis. This pilot study compared the performance of a prototype lightwand device (LW) with the Glidescope ${ }^{\circledR}$ (GS) in a cadaver airway model with simulated oropharyngeal bleeding.

Methods: Ethics approval was obtained from the local REB. This randomized control trial used clinical grade cadavers, approved for medical teaching and research purposes at QEII/Dalhousie University Skills Centre. To simulate an active oropharyngeal bleed, expired packed red blood cells were continuously instilled through each cadaver's nostril. Six Anesthesia staff were recruited and block randomized to intubate the trachea of the cadavers. An orientation video to the LW was provided prior to the study. The primary outcome measure was Time-to-Intubation (TTI), and the secondary outcome measures included Time-to-AirwayManagement (TAM), number of attempts, and subjective degree of difficult intubation (DDI) by visual analog scale (VAS). Using power analysis, a sample size of 17 intubations was estimated to be adequate for each device (34 intubations total). 48 intubations were recorded and reviewed. Linear Mixed Models were used to analyze the data.

Results: Overall, the LW offered comparable performance regarding average TTI (95\% Cl [$17.2,4.74], \mathrm{p}=0.273)$ and TAM $(95 \% \mathrm{Cl}[-19,4.51], \mathrm{p}=0.234)$. The DDI was the only outcome that was significantly different. On average, participants rated the LW device (mean $=26.5 \mathrm{~mm}$ ) higher than the GS (mean $=16 \mathrm{~mm}$ ) on their VAS $(95 \% \mathrm{Cl}[-19.4,-1.54], \mathrm{p}=0.027)$. Of note, a loose right maxillary central incisor was observed after one GS intubation attempt.

Conclusion: This pilot study examined both video laryngoscopy and the LW technique in a simulated bleeding airway model. The results of this study showed that the prototype LW and GS have a comparable likelihood of successful airway management in patients with oropharyngeal bleeding. While the results suggested a higher DDI for LW, it should be noted that all staff participating in the study were experienced in the use of the GS, but not the LW device. It is unclear if the prototype LW is less likely to cause dental trauma - further investigation is warranted.

\section{REFERENCES:}

1. Sakles JC, Corn GJ, Hollinger P, Arcaris B, Patanwala AE, Mosier J. Acad Emerg Med. 2017; 24: 628-636 


\section{Language Modification to Reduce Anxiety for Awake Intubation in High School Students}

Timothy P. Turkstra ${ }^{1}$; Anna K. Turkstra²; Jayden A. Graystone ${ }^{2}$; Mariana I. Moreno ${ }^{2}$

1 University of Western Ontario, London, ON, Canada

2 London Central Secondary School, London, ON, Canada

Introduction: Discussion of a potential awake intubation during the pre-admit consultation can provoke anxiety. After recognising that occasionally the word "awake" specifically provoked anxiety, we decided to investigate other descriptions that could be less intimidating and/or anxiety-provoking, but still accurate. We hypothesised that focusing on the sedation and the local anesthesia could reduce anxiety, much as it does in dental practice.

Methods: Local ethics approval was obtained for the study which, as an anonymous survey, did not involve patient records. Surveys were distributed to students to determine their comfort level with a proposed awake intubation. The survey and individual component questions were voluntary and uncompensated. Potential participants were provided a link to participate on their mobile device. The survey also included demographic questions and distractor questions.

The respondents were randomised to receive different surveys; both scenarios described identical surgical procedures and post-op care, but they described the awake intubation with different descriptors. In the sedated/freezing description group (group SFD), the description focussed on the sedation. In the control group (group AIF), the intubation used the word "awake" and described the procedure and local anesthesia.

Respondents were queried with respect to their comfort/anxiety the procedure for a family member and for themselves personally. Respondents scored their comfort level on a linear virtual slider $(0 \%=$ great anxiety, $100 \%=$ complete comfort $)$. Multiple responses were not permitted.

Results: The survey was distributed to 1011 people, and was returned by 387, for a response rate of $38 \%$. The average age was $16+/-1$ years old. Of those who provided their gender, $55 \%$ identified as female, $45 \%$ male, $0.5 \%$ other. Demographics were similar between the two groups.

In group SFD, respondents rated their comfort level at $45.3 \%+/-30.8 \%$ (mean +/- SD) for a family member and $47.9 \%+/-32.9 \%$ for themselves personally. In group AIF, respondents scored their comfort level at $45.2 \%+/-33.1 \%$ for a family member and $47.0 \%+/-32.5 \%$ for themselves personally. See figure. There was no significant difference between the groups based on the descriptions of the intubation ( $p=0.95, p=0.79)$ nor whether it was a family member or themselves undergoing the procedure $(p=0.48)$

Interpretation: Using less intimidating language did not reduce the anxiety provoked when considering awake intubation in this population. It is possible that other aspects of the surgical description had a greater contribution to perceived anxiety than the awake intubation description. 
The response rate was impressive for a survey study. Application of the study to the general Canadian population could be limited by the young age of the respondents, who might be more or less comfortable with awake intubation than the general population.

\section{Comfort Level with Awake Intubation}

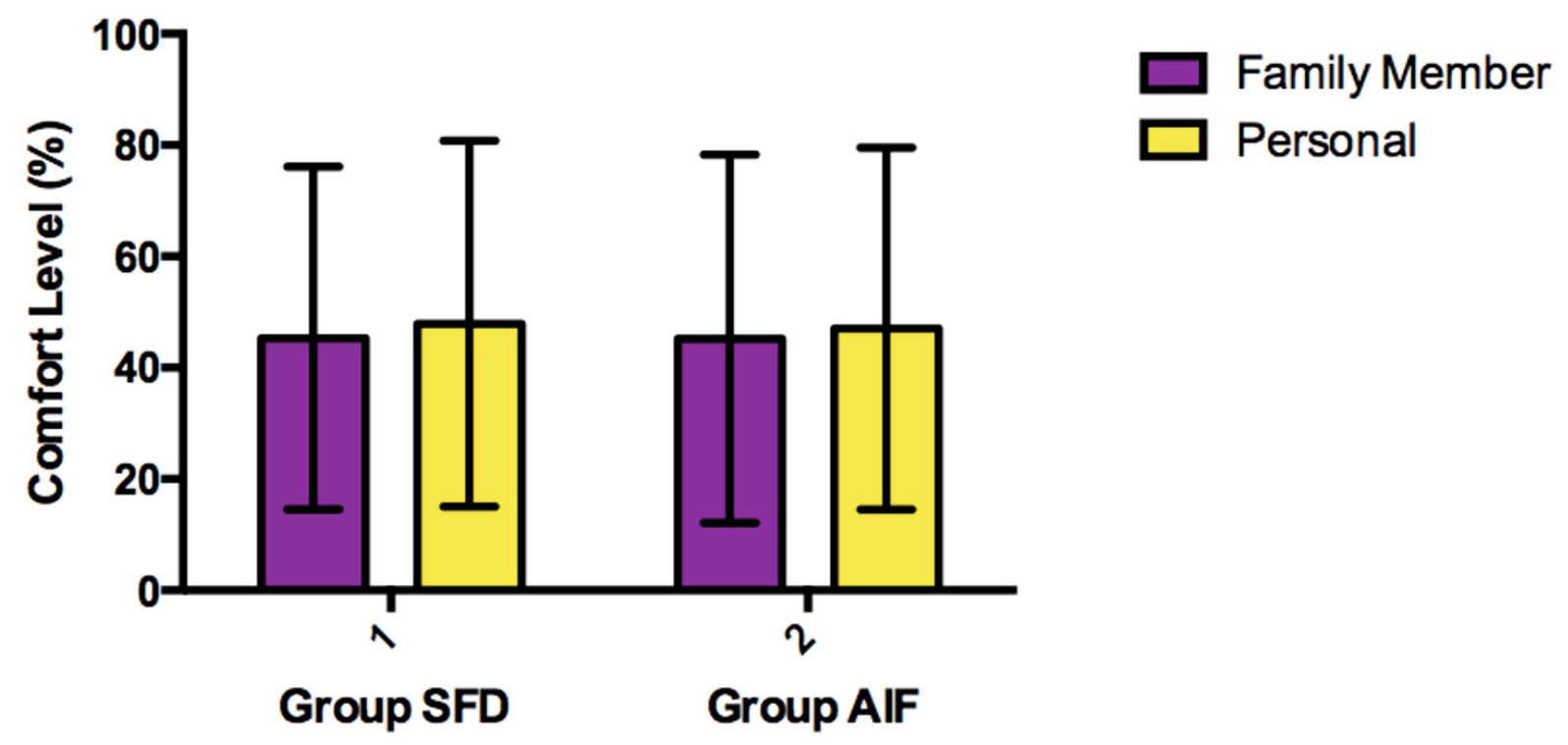


McKay Airway: Exploratory Study of a Novel Oral Airway for Bag-Mask Ventilation

William P. McKay ${ }^{1}$; Udoka Okpalauwaekwe ${ }^{2}$; Cuylar Conly ${ }^{3}$; Bhavna Pooni ${ }^{4}$

1 Dept of Anesthesia, University of Saskatchewan, Saskatoon, Canada

2 Division of Cardiology, University of Saskatchewan, Saskatoon, Canada

3 University of Saskatchewan College of Medicine, Saskatoon, Canada

4 Faculty, Perioperative Services and Medical Device Reprocessing, Saskatchewan

Polytechnic, Saskatoon, Canada

Introduction: We studied a new oral airway device, the McKay Airway (MA). It is designed for easy maintenance of jaw-thrust for first responders who perform bag-mask ventilation (BMV) infrequently, and may not perform it well.1,2 Objective was to compare learners' time to achieve exhaled tidal volume (VE) $>300 \mathrm{ml}$ using MA versus using a Guedel oropharyngeal airway (OPA) for BMV on anesthetized patients. 3

Methods: Ethics approval was obtained from the local REB. We used a repeated-measures unblinded clinical trial with blinded randomization of airway use order. Anesthesiologists' decision to intervene and comments were recorded. Consenting BMV participants were naïve learners scheduled in the operating rooms to be taught airway management. Patients were consenting adults booked for elective surgery under general anesthesia. Excepted were those with loose incisors, temporomandibular joint disorders, reflux, or whom the attending anesthesiologist felt participation put them at added risk. For patients with missing teeth, we padded the MA surface with $1 \mathrm{~cm}$ thick stick-on sponge.

Following normal preoxygenation, induction of anesthesia, and BMV by the attending anesthesiologist as needed to assure high oxygen saturation, an opaque envelope was opened to randomly choose which airway to use first. We mimicked a self-inflating BMV device with the anesthetic machine by setting the Adjustable Pressure Limiting valve to $>30 \mathrm{~cm}$ water pressure and using the oxygen flush to keep the reservoir bag filled between breath attempts. With airways in place, learners applied the mask with their left hand, squeezing the bag with their right approximately every $3 \mathrm{~s}$. We timed from first squeeze until VE exceeded $300 \mathrm{ml}$, then changed to the other airway.

Results: We recruited 34 female and 20 male patients, and obtained data for 51 . One obese patient had severe succinylcholine fasciculations with rapid profound desaturation and the anesthesiologist took over immediately and intubated; for two the anesthesiologist misunderstood the experiment. Continuous data were analysed with Wilcoxon's Signed Rank test, incidences with Chi Square. MA allowed ventilation 2 breaths sooner than OPA (HodgesLehmann median difference: $6 \mathrm{~s}$ [95\% $\mathrm{Cl} 5$ to $6.3 \mathrm{~s}$ ]; range: OPA 5 to $78 \mathrm{~s}$; MA 2 to $49 \mathrm{~s} ; \mathrm{p}=$ 0.02 ). Comments: 16 preferred the MA, 2 the OPA ( $p=0.02$ compared to equal preferences), and 15 had no preference.

Overall the second attempt was faster, as learners became more familiar with BMV technique, suggesting that there is a learning effect. The second trial was faster than the first 31 [25 to 37] times compared to only $20[16$ to 24$]$ times when the first trial was faster $(p=0.047)$. Despite this, when used first, the MA was faster than the OPA $30 \%$ of times compared to $11 \%$ when OPA was used first $(p=0.04)$, suggesting that MA is easier to learn.

Conclusion: Further studies are warranted. 


\section{REFERENCES:}

1. Soleimanpour H, Gholipouri C, Panahi JR, Afhami MR, Ghafouri RR, Golzari SE, Soleimanpour M, Esfanjani RM: Role of anesthesiology curriculum in improving bag-mask ventilation and intubation success rates of emergency medicine residents: a prospective descriptive study. BMC Emerg Med 2011; 11: 8

2. Timmermann A, Russo SG, Crozier TA, Eich C, Mundt B, Albrecht B, Graf BM: Novices ventilate and intubate quicker and safer via intubating laryngeal mask than by conventional bag-mask ventilation and laryngoscopy. Anesthesiology 2007; 107: 570-6

3. Baskett TF: Arthur Guedel and the oropharyngeal airway. Resuscitation 2004; 63: 3-5

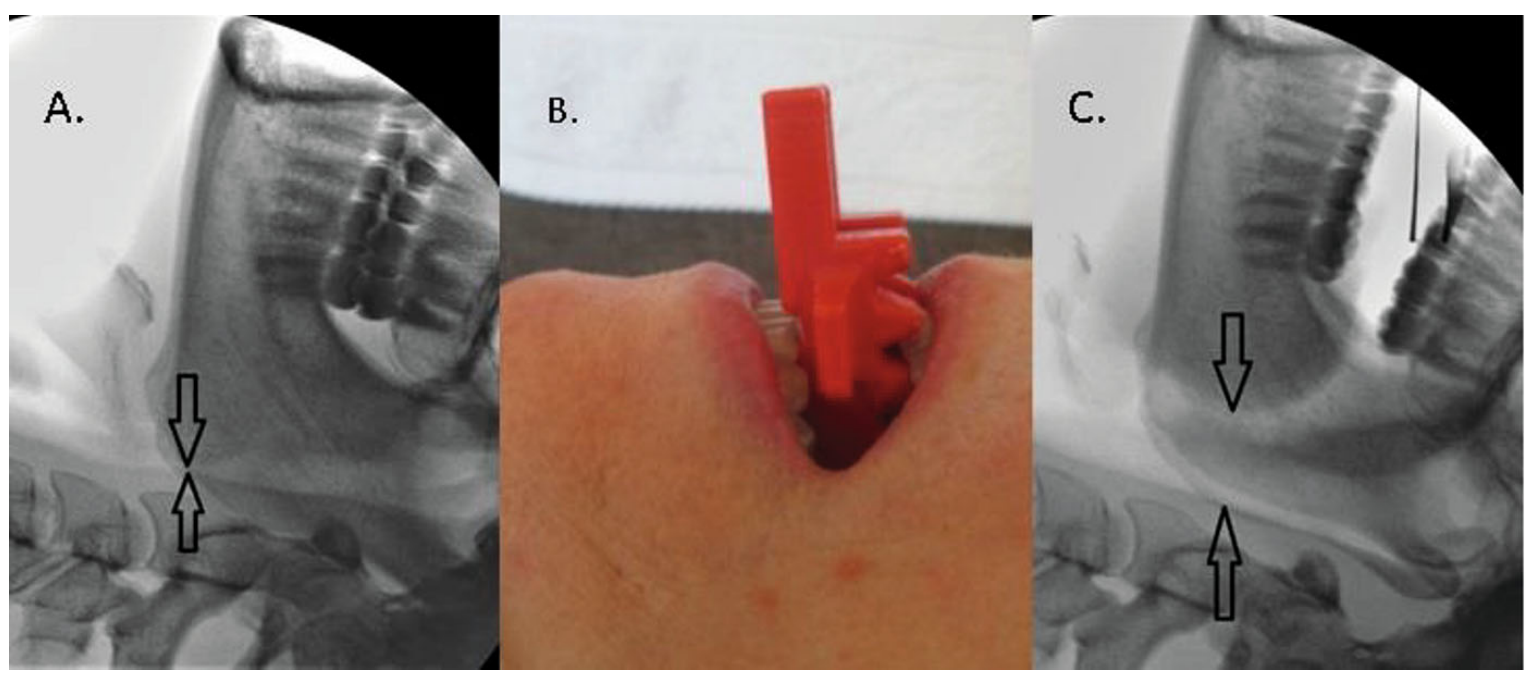




\section{Somatosensory Evoked Potentials Monitoring During Intubation in a Patient with Unstable Cervical Spine}

\section{Dam-Thuy Truong ${ }^{1}$, Ravish Kapoor ${ }^{1}$, Angela Truong ${ }^{1}$}

1 Department of Anesthesthesiology and Perioperative Medicine, University of Texas MD Anderson Cancer Center, Houston, Texas, USA

Introduction: Airway management is the most critical aspect of anesthesia for patients with unstable cervical spine. The best monitor of spinal cord integrity during intubation is an awake patient who can report neurological symptoms to the anesthesiologist, thus preventing irreversible damage to the spinal cord. ${ }^{1}$ In case of patient refusing awake intubation, somatosensory evoked potentials (SSEP) monitoring provides a valuable alternative technique to reliably detect spinal cord injury during airway manipulation. The use of SSEP monitoring for intubation has never been previously described.

Case Presentation: The patient gave consent for publication of this case. A 63-year-old female with breast cancer presented for mastectomy and breast reconstruction. Past medical history was significant for rheumatoid arthritis involving the joints of the hands, feet, and cervical spine. She had no neurological symptoms. Medications included methotrexate and tramadol. Physical examination revealed an obese female with weight $73 \mathrm{~kg}$, height $143 \mathrm{~cm}$, and BMI 35 $\mathrm{kg} / \mathrm{m} 2$. She had a Mallampati class III airway and severely restricted neck extension. Preoperative cervical spine radiographs showed a widened gap between the odontoid and anterior ring of $\mathrm{C} 1$. Cervical spine MRI (A) confirmed marked atlantoaxial subluxation shown as separation between the anterior arch of $\mathrm{C} 1$ (red arrow) and the dens (purple arrow). The plan was to perform an awake fiberoptic intubation. Despite extensive discussion, the patient adamantly refused.

Neurosurgical consultation was obtained and electrophysiologic monitoring was recommended as an alternative to assess spinal cord integrity. In the operating room, the neck was placed in neutral position with a rigid cervical collar to provide support and immobilization in all planes of neck motion. Stimulating electrodes were placed over the median, ulnar and tibial nerves. SSEPs were recorded from scalp electrodes over the somatosensory cortex. Baseline recordings of latency, rate and amplitude of the evoked components were noted. Anesthesia was induced with fentanyl and propofol. Once bag-mask ventilation was assured, rocuronium was administerd and the trachea intubated with a flexible bronchoscope. The neck was maintained in neutral position throughout ventilation and intubation (B). SSEP monitoring did not detect any signs of neurological injuries. Maintenance of anesthesia was achieved with 0.5 MAC inhalational anesthesia, propofol infusion $50-125 \mathrm{mcg} / \mathrm{kg} / \mathrm{min}$, and standard doses of opioids and muscle relaxants. Boluses of medications were avoided to maintain a steady state for optimal SSEP readings. Surgery and recovery were uneventful.

Discussion: SSEP monitoring has long been used during neurosurgical procedures to detect and prevent spinal cord injury. ${ }^{2}$ Reliable, safe, and readily available, it allows assessment of sensory pathways and global functioning of the spinal cord. For patients with unstable cervical spine such as our patient with rheumatoid arthritis refusing awake intubation, SSEP monitoring 
should be considered as a valuable modality for monitoring during airway manipulation under general anesthesia.

\section{REFERENCES:}

1. Acta Anaesthesiol Scand 2019;00: 1-12

2. Anaesthesia 2000; 55(3): $225-41$

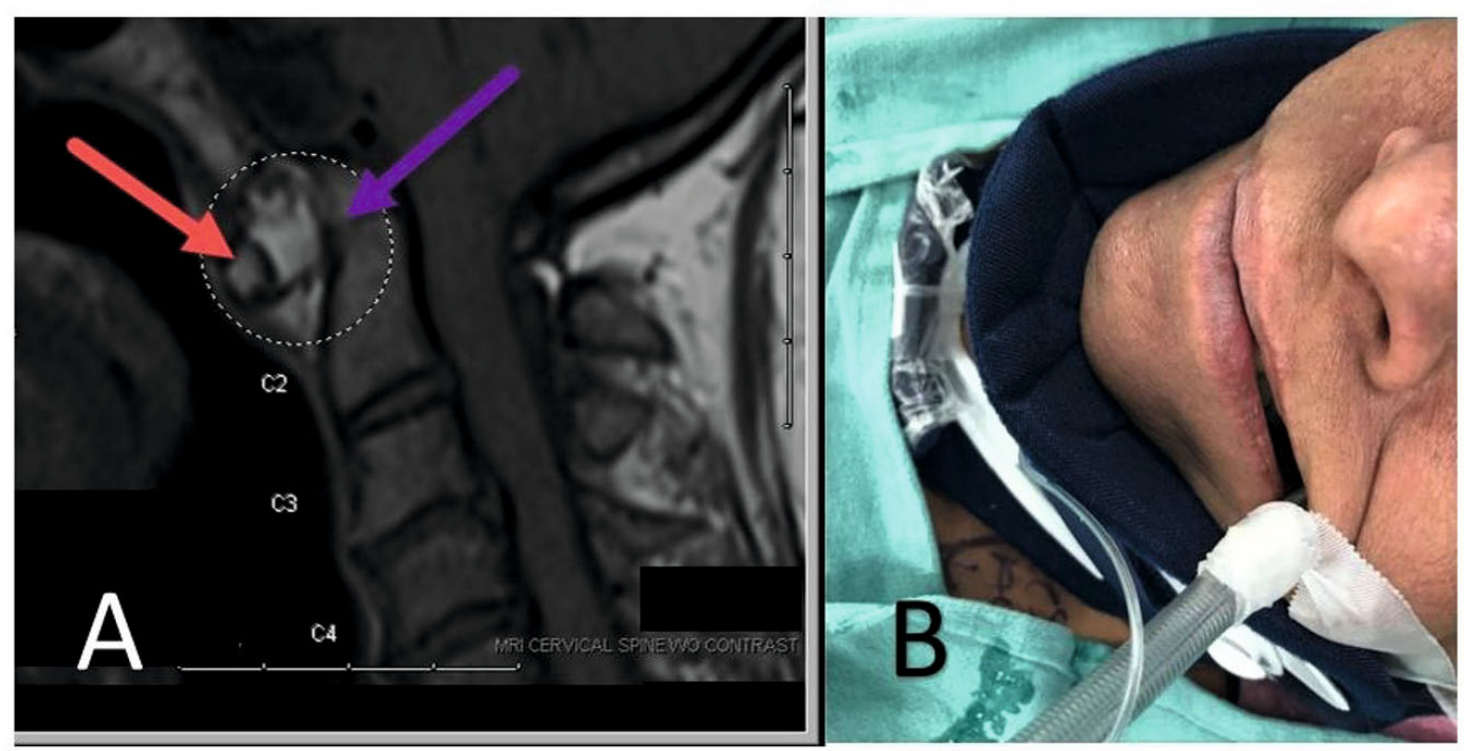




\title{
AMBULATORY
}

\section{Incidence, Risk Factors and Outcomes of Perioperative Cardiac Arrhythmias Following} Non-Cardiothoracic Surgery: A Systematic Review and Meta-Analysis

\author{
Omar El Tohamy ${ }^{1}$; Yamini Subramani ${ }^{2}$; Daniil Jalali ${ }^{3}$; Mahesh Nagappa ${ }^{2}$; Homer Yang ${ }^{2}$; Ashraf $^{2}$ \\ Fayad $^{2}$
}

1 Department of Medical Sciences, Schulich Medical Sciences, Western University, London, Ontario, Canada

2 Department of Anesthesia and Perioperative Medicine, University Hospital, Western University, London, Ontario, Canada

3 Department of Medical Sciences, Schulich Medical Sciences and Ivey Business

School, Western University, London, Ontario, Canada

Introduction: Perioperative supraventricular arrhythmias are common after non-cardiothoracic surgeries and can lead to serious perioperative complications. 1 The pathophysiology of postoperative AF after non-cardiothoracic surgery is poorly understood, but is thought to be due to stress and inflammatory postoperative response. 2 There is presently limited evidence concerning new-onset arrhythmias (AF) following non-cardiothoracic surgery. The primary objective of this systematic review and meta-analysis (SR and MA) was to identify the risk factors of new-onset arrhythmias during non-cardiothoracic surgery.

Methods: Ethics approval was not applicable because the study did not involve human or animal research. We included studies which reported on any new-onset perioperative arrhythmia in adult patients (>18 years) after non-cardiothoracic surgery. Those studies without explicit reporting of perioperative arrhythmias were excluded. We searched multiple databases according to a pre-defined systematic search strategy. The primary outcome was to determine the demographic and clinical risk factors for new-onset perioperative arrhythmias after noncardiothoracic surgery. Secondary outcomes were the incidence of perioperative complications associated with the arrhythmias. Data on demographics, comorbidities and perioperative complications were analysed and reported as corresponding crude odds ratios (OR) and 95\% confidence intervals $(\mathrm{Cl})$. Random-effects models was used to estimate the pooled odds ratios for risk of perioperative complications. A two-sided $P$ value less than 0.05 was considered statistically significant. Impact of heterogeneity (I2) was assessed by calculating the 12 statistic.

Results: Fifteen studies met the inclusion criteria, reporting on 123,157 patients undergoing non-cardiothoracic surgery, of which 3,087 developed perioperative arrhythmias. The pooled incidence of postoperative arrhythmia was calculated as 2.51 (95\% Cl 2.42-2.60). Advanced age (MD: 4.21; 95\% Cl: 2.15 to 6.28 ; I2: 89\%; p<0.0001).(20-34), preoperative hypertension (Perioperative Arrhythmia vs Control: $60.0 \%$ vs $56.7 \%$; OR: $1.53 ; 95 \% \mathrm{Cl}: 1.06$ to $2.22 ; 12: 52 \%$; $\mathrm{p}=.009$ ), pre-existing cardiac disease (Perioperative Arrhythmia vs Control: $25.3 \%$ vs 4.9\%; OR: 2.26; $95 \% \mathrm{Cl}: 1.19$ to $4.30 ; 12: 89 \% ; \mathrm{P}=0.003$ ) and a history of atrial fibrillation (Perioperative Arrhythmia vs Control: 20.5\% vs 3.9\%; OR: 5.60; 95\% Cl: 2.64 to 11.89; $2: 51 \%$; P<0.00001) were found to be significant predictors for perioperative arrhythmia The development of perioperative arrhythmia was significantly associated with development of post-operative 
respiratory complications (OR: 3.69; 95\% Cl: 1.50 to 9.11; I2: 80\%; P=0.005).(22,29,31,34), cardiac complications (Perioperative Arrhythmia vs Control: $34.1 \%$ vs 5\%; OR: $5.24 ; 95 \%$ Cl: 2.78 to 9.85; I2:69\%; P<0.00001) and increased mortality (Perioperative Arrhythmia vs Control: $11.3 \%$ vs $2.4 \%$; OR: 6.31 ; $95 \% \mathrm{Cl}: 3.19$ to 12.50 ; I2:33\%; $\mathrm{P}<0.00001)$.

Conclusion: This SR and MA identified advanced age, male gender, pre-existing cardiac comorbidities, hypertension and history of atrial fibrillation as risk factors associated with perioperative arrhythmia. We also found that the arrhythmia group had an increased postoperative cardio-pulmonary complications and higher mortality compared to the nonarrhythmia group, emphasizing the need for risk stratification and close monitoring of this surgical population.

\section{REFERENCES:}

1. Kahn RL, Hargett MJ, Urquhart B, Sharrock NE, Peterson MG. Supraventricular tachyarrhythmias during total joint arthroplasty. Incidence and risk. Clin Orthop Relat Res.1993 Nov;(296):265-9.

2. Chung MK, Martin DO, Sprecher D, Wazni O, Kanderian A, Carnes CA, et al. C-reactive protein elevation in patients with atrial arrhythmias: Inflammatory mechanisms and persistence of atrial fibrillation. Circulation. 2001 Dec 11;104(24):2886-91. 
Meta-analysis evaluating the risk factors of perioperative arrhythmias in patients undergoing non-cardiothoracic surgery

\begin{tabular}{|c|c|c|c|c|}
\hline Risk factor & Arrhythmia & Control & Pooled estimate & \\
\hline Age: Mean (SD) & $73.5(6.37)$ & $\begin{array}{l}67.4 \\
(4.54)\end{array}$ & & \\
\hline & & & -10 & 10 \\
\hline Male & $53 \%$ & $43.8 \%$ & & \\
\hline & & & 0.5 & 2 \\
\hline BMI: Mean (SD) & $26.67(5.52)$ & $\begin{array}{l}24.97 \\
(3.98)\end{array}$ & & \\
\hline DM & $22.27 \%$ & $22.75 \%$ & & \\
\hline & & & 0.5 & 2 \\
\hline Hypertension & $58.27 \%$ & $55.78 \%$ & & \\
\hline & & & 0.5 & 2 \\
\hline Cardiac disease & $25.3 \%$ & $4.9 \%$ & & \\
\hline & & & 0.1 & 10 \\
\hline $\begin{array}{l}\text { Respiratory } \\
\text { disease }\end{array}$ & $17.3 \%$ & $9.1 \%$ & & \\
\hline & & & 0.1 & 10 \\
\hline H/O Arrhythmia & $20.5 \%$ & $3.9 \%$ & & \\
\hline & & & 0.1 & 10 \\
\hline $\begin{array}{l}\text { MELD Score: } \\
\text { Mean (SD) }\end{array}$ & $29.63(9.9)$ & $\begin{array}{l}25.18 \\
(11.9)\end{array}$ & & \\
\hline & & & -2 & 2 \\
\hline
\end{tabular}


Perspectives of the Opioid Epidemic and Interventions to Improve Prescribing and Usage: A Cross Sectional Survey of Adult Ambulatory Orthopedic Surgical Patients and Their Health Care Providers

Luke Vanderhooft $^{1}$; Julie Mistri ${ }^{2}$; Peter MacDonald ${ }^{3}$; Scott Wolfe ${ }^{1}$; Kayla Kilborn ${ }^{1}$; Rachel Roy ${ }^{1}$; Thomas Mutter ${ }^{1}$

1 Max Rady College of Medicine Department of Anesthesiology, Perioperative and Pain Medicine, University of Manitoba, Winnipeg, Canada

2 Pharmacist, PRIME Care, Winnipeg Regional Health Authority, Winnipeg, Canada

3 Max Rady College of Medicine Section of Orthopaedic Surgery, University of Manitoba, Winnipeg, Canada

Introduction: High case volumes combined with excessive postoperative opioid prescriptions have made ambulatory surgery a significant contributor to the prescription opioid epidemic (POE). ${ }^{1}$ Single institutions have successfully reduced the quantity of dispensed postoperative opioid in select populations ${ }^{2-4}$ but widespread implementation is lacking. Sustainable interventions to ensure proper disposal and prevent misuse are also needed. A thorough understanding of stakeholder perspectives of the POE and proposed harm reduction strategies is a necessary precursor to achieving these broader goals. This survey assessed patient and health care provider (HCP) awareness of the POE, opioid related practices, behaviors and attitudes, and perceived barriers to the implementation of published harm reduction interventions. The goal was to inform a multifaceted, multidisciplinary intervention to prevent opioid misuse in ambulatory orthopedic surgery patients.

Methods: Ethics approval was obtained from the local REB. Five stakeholder groups participated in this cross-sectional, self-administered, anonymous online survey between January and May 2019. We invited all pharmacists in our province through a college newsletter and all anesthesiologists and orthopedic surgeons in our health region through departmental email lists. We also recruited a consecutive series of preoperative ambulatory orthopedic surgery patients and all postoperative care nurses at our ambulatory surgery facility. Survey content was tested for readability and informed by a literature search and stakeholder input. Questions were either specific to a stakeholder, common across stakeholders or common to HCPs. Analysis was descriptive.

Results: 165/225 (73\%) patients, 92/151 (61\%) anesthesiologists, 30/39 (77\%) orthopedic surgeons, $112 / 1632(7 \%)$ pharmacists and $8 / 8$ nurses participated. The response rate for each question was $\geq 80 \%$. Most patients and HCPs (range $86-100 \%$ ) were aware of the POE but $>40 \%$ in each group underestimated the reported $6 \%$ risk of persistent postoperative opioid use. ${ }^{1}$ The POE was seen as at least moderately important for minor surgery patients by $45 \%$ of patients and $50 \%$ (nurses) to $96 \%$ (surgeons) of HCPs. $58 \%$ of patients were male, $7 \%$ reported a household income less than $\$ 30,000 \mathrm{CDN}$ and $15 \%$ and $22 \%$ were $18-29$ and $>60$ years old, respectively. $39 \%$ of patients had opioids at home, $21 \%$ had used opioids for a nonprescribed indication, $9 \%$ had shared with others, $1 \%$ had used recreationally and at least $30 \%$ could not identify a safe disposal method from a list. Patients wanted more information about opioid misuse, multimodal analgesia, and postoperative weaning of analgesics delivered preferably on paper $(62 \%)$ or through conversation with specific HCPs (range $28-51 \%$ ) as opposed to through electronic media (14\%). Each HCP discipline endorsed having at least a moderately important 
role in ensuring safe postoperative opioid use (range 83-95\%). HCP specific interventions varied in perceived feasibility (Table 1 ).

Conclusion: Patients and health care providers have identified a number of potential targets for educational and process-of-care interventions.

\section{REFERENCES:}

1. Brummett, Chad M.Bohnert ASB, Waljee JF, Nallamothu BK, et al (2017) New Persistent Opioid Use After Minor and Major Surgical Procedures in US Adults. JAMA Surg 152:e170504. https://doi.org/10.1001/jamasurg.2017.0504

2. Hartford LB, Van Koughnett JAM, Murphy PB, et al (2019) Standardization of Outpatient Procedure (STOP) Narcotics: A Prospective Non-Inferiority Study to Reduce Opioid Use in Outpatient General Surgical Procedures. J Am Coll Surg 228:81-88.e1. https://doi.org/10.1016/j.jamcollsurg.2018.09.008

3. Hartford LB, Van Koughnett JAM, Murphy PB, et al (2019) The Standardization of Outpatient Procedure (STOP) Narcotics: A Prospective Health Systems Intervention to Reduce Opioid Use in Ambulatory Breast Surgery. Ann Surg Oncol 26:3295-3304. https://doi.org/10.1245/s10434-019-07539-w

4. Howard R, Waljee J, Brummett C, et al (2018) Reduction in Opioid Prescribing Through Evidence-Based Prescribing GuidelinesReduction in Opioid Prescribing Through EvidenceBased Prescribing GuidelinesLetters. JAMA Surg 153:285-287. https://doi.org/10.1001/jamasurg.2017.4436 


\begin{tabular}{|c|c|c|c|}
\hline Discipline & Intervention & Facilitators & Barriers \\
\hline Pharmacists & $\begin{array}{l}\text { Unused opioid } \\
\text { disposal }\end{array}$ & $\begin{array}{l}98 \% \text { of } \\
\text { pharmacies } \\
\text { accept opioids } \\
\text { for disposal }\end{array}$ & $\begin{array}{l}\text { - } 26 \% \text { do not communicate this to } \\
\text { patients verbally or in print materials } \\
\text { - } 35 \% \text { report lack of time } \\
\text { - Only } 2 \% \text { of pharmacists identified safe } \\
\text { "home-based" disposal techniques }\end{array}$ \\
\hline $\begin{array}{l}\text { Pharmacists \& } \\
\text { Postoperative } \\
\text { Care Nurses }\end{array}$ & $\begin{array}{l}\text { Patient education } \\
\text { on weaning opioids } \\
\text { and multimodal } \\
\text { oral analgesia }\end{array}$ & $\begin{array}{l}88 \% \text { of nurses } \\
\text { comfortable } \\
\text { describing } \\
\text { multimodal } \\
\text { regimens }\end{array}$ & $\begin{array}{l}\text { - Most pharmacists ( } 83 \%) \text { and nurses } \\
(62 \%) \text { believe }<60 \% \text { of patients leave } \\
\text { their care with a good understanding } \\
\text { - Lack of time, knowledge and resources } \\
\text { identified by at least } 20 \% \text { of respondents } \\
\text { in both groups }\end{array}$ \\
\hline Surgeons & $\begin{array}{l}\text { Reduced } \\
\text { prescription sizes }\end{array}$ & $\begin{array}{l}91 \% \text { see } \\
\text { surgeons as } \\
\text { having an } \\
\text { important } \\
\text { primary role in } \\
\text { this regard }\end{array}$ & $\begin{array}{l}\text { - } 38 \% \text { willing to reduce prescription sizes } \\
\text { - } 25 \% \text { want data on their patients first } \\
\text { - Increased office calls and visits } \\
\text { foreseen as significant problem by }>70 \%\end{array}$ \\
\hline Surgeons & $\begin{array}{l}\text { Prescribing opioids } \\
\text { not compounded } \\
\text { with } \\
\text { acetaminophen }\end{array}$ & $70 \%$ supportive & $\begin{array}{l}\text { - Triplicate opioid prescriptions create an } \\
\text { administrative burden on high volume } \\
\text { slates }\end{array}$ \\
\hline Surgeons & Partfill prescriptions & $79 \%$ supportive & $\begin{array}{l}\text { - Appropriate prescription sizes not } \\
\text { defined by the survey or in the literature }\end{array}$ \\
\hline Anesthesiologists & $\begin{array}{l}\text { Alter anesthetic for } \\
\text { patients at high risk } \\
\text { of misuse }\end{array}$ & $\begin{array}{l}89 \% \text { at least } \\
\text { somewhat likely } \\
\text { to do so }\end{array}$ & $\begin{array}{l}\text { - } 25 \% \text { have lack of knowledge or skill for } \\
\text { distal upper extremity blocks and lower } \\
\text { limb blocks }\end{array}$ \\
\hline
\end{tabular}

Table 1: Selected list of facilitators and barriers to harm reduction interventions to optimize postoperative opioid use. Prescribing opioids not compounded with acetaminophen facilitates regular use of multimodal analgesia. Partfill prescriptions limit the initial number of opioids dispensed and provide the option to return to the pharmacy for the remainder of the prescription if needed. 
Preoperative Needs and Postoperative Satisfaction in Patients Undergoing Elective Hip and Knee Surgical Procedures - A Quantitative Evaluation - The First Step in a Program of Research to Help Improve Patient Preparation for, and Outcomes with, Early PostOperative Home Recovery

Mahesh Nagappa ${ }^{1}$; Moaz Bin Yunus Chohan ${ }^{1}$; Yamini Subramani ${ }^{1}$; Kevin Armstrong ${ }^{1}$; Janet Martin ${ }^{2}$; Ava John-Baptiste ${ }^{2}$; Brent Lanting ${ }^{3}$; Christopher Schlachta ${ }^{4}$; Jullian Von Koughnett ${ }^{4}$; Kathy Speechley ${ }^{4}$; Ashraf Fayad ${ }^{1}$; Homer Yang ${ }^{1}$

1 Department of anesthesia and perioperative medicine, Western University, London, Ontario, Canada

2 Department of biostatistics and epidemiology, $\mathrm{MEDICI}$, And Department of anestheisia and perioperative medicine, Western University, London, Ontario, Canada

3 Department of orthopedics, Western University, London, Ontaion, Canada

4 Department of surgery and colorectal unit, Western University, London, Ontario, Canada

Background: It is the first step in a program of research that seeks to understand how well patients are prepared, in the preoperative phase, to understand the protocols, concerns and demands they will face post-surgery. Subsequently, understanding how well the needs of patients are met following surgery will be assessed by asking them about their access to support from family and other care givers, coping strategies, and level of satisfaction with their postoperative experience and health outcomes. 1

Objective: To investigate patients' concerns, preparation and expectations in the preoperative phase and the level of postoperative satisfaction in the perioperative period using a quantitative survey methodology.

Methods: 240 consecutive patients who attend the preadmission clinic for scheduled preoperative visits were invited, and informed consent was obtained. This study was conducted in accordance with the Declaration of Helsinki. Demographic and other clinical data was collected. Survey Questionnaires: Following recruitment, all patients were asked to respond to interviewer-administered survey questionnaire. Two interviews were conducted based on the study questions focussing on the objectives of the study. The first interview was conducted with the patient and with the patient's caregivers before the surgery and the second interview was conducted on postoperative day $5-7$ using the set of questions. The newly developed questions are designed based on the four potential areas of concerns for the early post-surgical discharge. 1 The preoperative questions seek to quantify the extent of patient knowledge, expectations, and preparedness to handle early post-surgical discharge. The postoperative questions seek to understand patient coping and management strategies post-surgery, to quantify how well the patient is doing. This was done by rating on a 5-point Likert scale (with the higher score indicating a higher level of patient need \& satisfaction).

Results: This prospective observational study explored the level of patients' needs and satisfaction undergoing the hip and knee surgical procedure, based on the patient-centred care framework. The patients had a mean age of $66 \pm 11$ years, $57 \%$ of the patients were female and $43 \%$ of the patients were male. $45.4 \%$ had the hip surgery and $54.8 \%$ had the knee surgery. The overall mean score for patients needs was $3.45 \pm 0.48$ and overall mean score for patient's satisfaction was $4.19 \pm 0.25$, out of possible 5 Lockhart scale. Among the subdomains of the 
patient need and satisfaction, the perioperative medication, pain management, recovery process and side-effects/complications were reported. Nearly $12.9 \%$ of the patients visited the family doctor within 5 days after the surgical procedure.

Conclusion: This study reports the higher level of patient need and higher level of satisfactions in patients undergoing the hip and knee surgical procedure. These results add to the body of the knowledge in understanding the patients' needs and satisfaction in the perioperative period.

\section{REFERENCES:}

1. LHSC. London Health Sciences Centre - Decision Support, Quality Based Payment (QBP) data For Years 15/16, 16/17. 


\title{
CARDIOVASCULAR AND THORACIC
}

\section{Frog-Breathing Pattern: Pathognomonic Sign of a Tracheo-Subcutaneous Fistula}

\author{
Angela Truong ${ }^{1}$; Dam-Thuy Truong ${ }^{1}$
}

1 Department of Anesthesiology and Perioperative Medicine, University of Texas MD Anderson Cancer Center, Houston, Texas, USA

Introduction: Surgical resection of thyroid cancer invading the trachea may cause injury to the tracheal wall, leading to tracheal fistula. ${ }^{1}$ We report a patient who developed an unusual breathing pattern after thyroidectomy with partial tracheal resection. A subcutaneous air pocket on his neck inflated with each expiration and deflated with inspiration. Because only a stable tracheal window which remained open throughout the respiratory cycle could explain this breathing pattern, a diagnosis of a tracheal leak was made.

Case Presentation: Patient consent was obtained for publication of this case. A 68-year-old man with invasive thyroid carcinoma and worsening hypoxemia presented for emergent surgical neck exploration. Two days prior, he had undergone thyroidectomy, neck dissection, and resection of the first two tracheal rings. Medical history included hypertension and type 2 diabetes mellitus. Vital signs showed BP 155/88, pulse $104 / \mathrm{min}, \mathrm{RR} 22 / \mathrm{min}, \mathrm{SpO}_{2} 87 \%$ with face mask oxygen at $8 \mathrm{~L} / \mathrm{min}$, and temperature 37.9 degrees Celsius. His face was flushed and he displayed use of respiratory accessory muscles. With each expiration, the left anterolateral side of his neck inflated into a circular air pocket about $10 \mathrm{~cm}$ in diameter $(A)$ which subsequently deflated $(B)$ during inspiration, reminiscent of the breathing pattern of a frog. After intubation, it was impossible to visualize the tracheal fistula by bronchoscopy because the tracheal tube obstructed the fistula. Only after surgical exposure of the trachea that a $1.5 \times 1.5$ $\mathrm{cm}$ anterolateral tracheal wall fistula was revealed. Severe inflammation of the area along with copious bronchial secretions precluded tracheal anastomosis. A tracheostomy tube was inserted to secure the airway and enable effective tracheobronchial toilet. Decannulation occurred 18 days later.

Discussion: Anatomically, the frog-breathing pattern can only occur when a defect remains patent throughout the respiratory cycle to allow not only the exit but also the re-entry of expired air back into the tracheobronchial tree and lungs. Cartilaginous tracheal rings are rigid enough to keep the opening patent. In contrast, a perforation of the lungs, pleura, pharynx and esophagus may cause air leak leading to pneumothorax, mediastinal and subcutaneous emphysema. ${ }^{2}$ Defects in these organs with flaccid walls collapse during inspiration and cannot account for the breathing pattern observed. Physiologically, the subcutaneous reservoir constituted a significant added dead-space from which rebreathing resulted in hypoxemia and hypercarbia manifested as tachycardia, cutaneous flushing and sweating. While expiration is mainly passive by chest wall recoil, inspiration requires greater effort to entrain air through a small opening. For every breath, the expired volume was slightly greater than the inspired volume. As the size of the air pocket enlarged, increased work of breathing precipitated respiratory failure. This repetitive characteristic frog-breathing pattern can make the diagnosis of a tracheo-subcutaneous fistula, even without confirmation by investigative diagnostic imaging. 


\section{REFERENCES:}

1. J Thorac Dis 2016 Mar;8 (Suppl 2): S160-7

2. Arch Intern Med 1984 Jul; 144(7): 1447-53

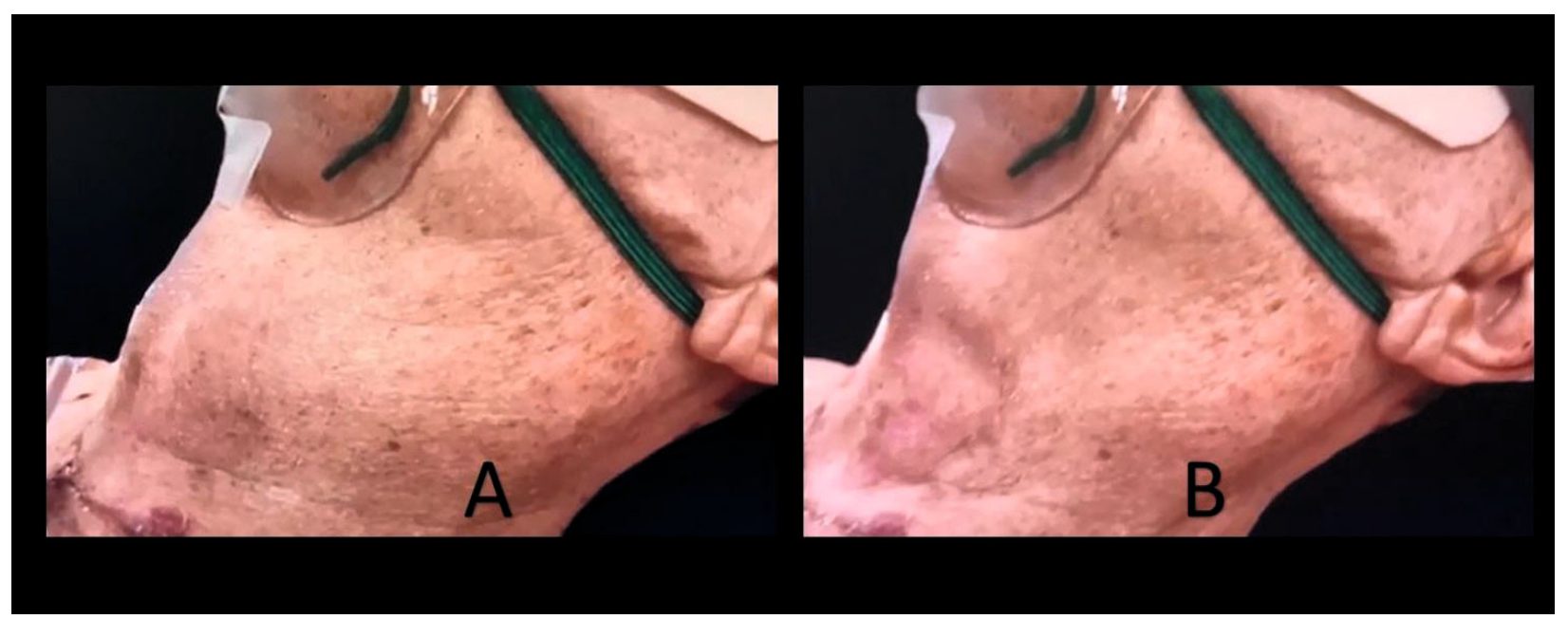




\section{Heparin Activity Reversal Trial (HART): A Qualitative Analysis of (Anti)Coagulation Practices Among Cardiac Anesthesiologists at a Single Academic Centre}

Rachelle Lassaline ${ }^{1}$; Sayra Cristancho ${ }^{2,3,4}$; Zachary Hynes ${ }^{5}$; Ravi Taneja ${ }^{5,6}$

1 Schulich School of Medicine and Dentistry, Western University, London, Ontario, Canada

2 Centre for Education Research and Innovation, Schulich School of Medicine and

Dentistry, Western University, London, Ontario, Canada

3 Department of Surgery, Schulich School of Medicine and Dentistry, Western University, London, Ontario, Canada

4 Faculty of Education, Western University, London, Ontario, Canada

5 Department of Anesthesiology and Perioperative Medicine, Schulich School of Medicine and Dentistry, Western University, London, Ontario, Canada

6 Division of Critical Care Medicine, Department of Medicine, Schulich School of Medicine and Dentistry, Western University, London, Ontario, Canada

Introduction: Clinical equipoise exists in the amount of protamine required to neutralize heparin during cardiac surgery. ${ }^{1}$ Surgeons commonly ask for more protamine if there is ongoing hemorrhage, but anecdotally, it appears that cardiac anesthesiologists hold different rationales to decide how much is too much. Optimal control of hemostatis remains obligatory at this moment in the operating room or else the resultant hemorrhage and transfusions can not only be resource intesnisve but also have adverse outcomes. ${ }^{2}$ Thus, the objective of this study was to explore the factors that influence cardiac anesthesiologists' decision-making for (anti)coagulation management in the cardiac surgery operating room. Understanding these may help increase homogeneity in clinical practice and may allow for development of written guidelines.

Methods: Ethics approval was obtained from the local REB. Following ethics approval and writen consent, 20 cardiac anesthesiology faculty members at a tertiary hospital setting were invited for semi-structured, in-depth, face-to-face interviews. Participants were asked questions about their practice of administering heparin and protamine. The interviews were transcribed and anonymized, then analyzed using NVivo (Version 12). Data collection and iterative anaylsis occured concurrently using Constructivist Grounded Theory methodology.

Results: Uncertainty about the required dose of protamine amongst anesthesiologsits was caused by paucity of evidence and lack of guidelines in clinical practice. Participants reported that absence of feedback about their postoperative patients in the Intensive Care Unit, as opposed to those transported to the Post Anesthesia Care Unit, impaired feedback on progress postoperatively. The learning culture at the institution was characterized by interprofessional collaboration with surgeons and perfusionists and dominated by peer influence. Appeasement with surgeons was common as were convenience and simplification strategies for (anti)coagulation management. Centre-specific practices evolved the local institution and these were influenced predominantly by available monitoring equipment (or lack thereof) as well as a dominant peer influence. Participants thought that development of written guidelines would decrease conflict, update faculty and would serve as an educational tool for trainees.

Discussion/Conclusion: Clinical equipoise in the management of (anti)coagulation during cardiac surgery can be associated with evolution of centre-specific practices. Development of 
written guidelines for administration of heparin and protamine is perceived to have numerous beneficial effects for both trainers and trainees.

\section{REFERENCES:}

1. Boer $\mathrm{C}$, Meesters $\mathrm{M}$, Veerhoek $\mathrm{C}$, et al. Anticoagulation and side effects of protamine in cardiac surgery: a narrative review. Br J Anaesth 2018. DOI: 10.1016/j.bja.2018.01.023

2. Schwann T, Habib J, Khalifeh J, et al. Effects of blood transfusion on cause-specific late mortality after coronary artery bypass grafting - less is more. Ann Thorac Surg 2016. DOI: http://dx.doi.org/10.1016/j.athoracsur.2016.05.023 


\title{
Hypersensitivity Reaction During Endovascular Abdominal Aortic Aneurysm Repair with
} a Low Profile Stent

\author{
Jillian C. Belrose ${ }^{1}$; Adam H. Power ${ }^{2}$; Katherine M. Ower ${ }^{1}$
}

1 Department of Anesthesia \& Perioperative Medicine, Schulich School of Medicine \& Dentistry, University of Western Ontario, London, Ontario, Canada

2 Department of Vascular Surgery, Schulich School of Medicine \& Dentistry, University of Western Ontario, London, Ontario, Canada

Introduction: Eligibility for endovascular aneurysm repair (EVAR) can be limited by narrow access vessels or aneurysm neck anatomy. To address this limitation, novel low-profile stents have been developed. The Ovation Abdominal Stent Graft System (Endologix Inc., Irvine, CA) lacks the nitinol support which reduces the profile to a $14 \mathrm{~F}$ outer diameter. Once the graft is deployed, inflatable rings are filled with a radiopaque polyethylene glycol (PEG)-based polymer. Deployment of the graft has been associated with polymer leak in 1/400 patients [1, 2]. Here, we present a case of profound hypotension due to polymer leak.

Case Presentation: Patient consent was obtained for publication of this case. A 67-year old male presented for EVAR to manage an abdominal aortic aneurysm (AAA). CT imaging revealed a $6.6 \mathrm{~cm}$ infrarenal $A A A$, severe atherosclerotic disease in the common iliac arteries, and thrombus within the aneurysm neck. The surgery was performed under general anesthesia with endotracheal intubation, a large bore IV, and arterial line. The Ovation stent was inserted and an angiogram was used to position the graft. The stent was deployed and polymer was injected. Approximately 12 minutes following polymer injection, the patient became profoundly hypotensive (BP 55/42) and tachycardic. Respiratory parameters and ECG were unchanged. There was no visible rash or wheeze. No hematoma or other sign of hemorrhage was identified. Anaphylaxis management was initiated including epinephrine, hydrocortisone, diphenhydramine, and IV fluid resuscitation. Diagnosis of polymer leak was confirmed. Four minutes following the initial reaction, the contralateral side was cannulated and a stiff wire was advanced through the graft. A Reliant stent graft balloon was then used to occlude the aorta in the supraceliac position. This was left inflated for 5-10 minutes, and slowly deflated once a perfusing blood pressure was achieved. Serum trypase at the time of the reaction and at the 1 hour timepoint were within normal limits. At the end of the case, vasopressors were no longer required and the patient was transferred to the ICU. He was discharged home on post-operative day 3.

Discussion: The risk of a polymer leak and hypersensitivity reaction with the Ovation graft is approximately $1 / 400$. In the reported cases, $24 / 26$ developed hypotension [2]. This reaction may be independent of $\mathrm{IgE}$ as tryptase was not elevated in our case. An anaphylactoid reaction should be considered with acute onset hypotension after polymer injection. Treatment should include management for anaphylaxis, and consideration of balloon occlusion of the aorta during resuscitation. To improve safety, the type of stent should be included in the surgical pause and injection of the polymer should be announced by the surgical team. We recommend arterial line access and large bore IV or central line access in all patients undergoing EVAR with a lowprofile stent. 


\section{REFERENCES:}

1. Sfyroeras GS, Moulakakis KG, Antonopoulos CN, Manikis D, Vasdekis SN. Anaphylactic Reaction During Implantation of the Ovation Stent-Graft System in a Patient With Abdominal Aortic Aneurysm. J Endovasc Ther Off J Int Soc Endovasc Spec. 2015;22:6202.

2. Gupta N, Hynes KL, Mahrouyan O, Briggs CS, Azzizadeh A. Polymer leak with the Ovation Abdominal Stent Graft System: Early recognition and treatment. Vascular. $2019 ;: 1708538119862923$. 
Implementation of an Enhanced Recovery After Thoracic Surgery (ERATS) Program at an Academic Center

Calvin Thompson ${ }^{1}$; Stephane Moffett ${ }^{1}$; Amanda Mattice ${ }^{2}$; Yaseen Al Lawati ${ }^{2}$; Nazgol Seyednejad"; Jan Leahy ${ }^{3}$; Caitlin Anstee ${ }^{2}$; Edita Delic ${ }^{2}$; Anna Fazekas²; Donna E. Maziak ${ }^{2}$; P James Villeneuve ${ }^{2}$; Sebastien Gilbert ${ }^{2}$; Farid Shamji ${ }^{2}$; R Sudhir Sundaresan ${ }^{2}$; Sylvain Gagne ${ }^{1}$; Andrew Seely ${ }^{2}$

1 Department of Anesthesiology and Pain Medicine, The Ottawa Hospital, Ottawa, Ontario, Canada

2 Department of Surgery, Division of Thoracic Surgery, The Ottawa Hospital, Ottawa, Ontario, Canada

3 Nursing, The Ottawa Hospital, Ottawa, Ontario, Canada

Introduction: Lung cancer is the leading cause of cancer death in Canada ${ }^{1}$. Thoracic surgical procedures are associated with significant morbidity, mortality and hospital resources. Targeted initiatives to optimize care should be sought to minimize adverse events (AEs), length of stay (LOS), and improve flow of care. Enhanced recovery after surgery (ERAS) initiatives have demonstrated impact in multiple surgical domains; however, data on thoracic surgery is sparse, with only recent recommendations being made $^{2}$.

Objectives: To evaluate the effects of implementing a Division wide comprehensive Enhanced Recovery After Thoracic Surgery (ERATS) program on postoperative outcomes, processes of care, patient experience, and quality of life (QOL).

Methods: Ethics approval was obtained by REB. We conducted a prospective, longitudinal study evaluating 9 months pre and 9 -months post implementation of an ERATS program. All patients undergoing the following procedures were included: anatomic pulmonary resection, esophagectomy, gastrectomy and giant paraesophageal hernia repair. Postoperative outcomes measured were: LOS, AEs, 30-day ER visits and readmissions. Process of care outcomes included time to: 'out-of-bed', independent ambulation, successful fluid intake, last chest tube removal, urinary catherization, removal epidural analgesia, discontinue patient-controlled analgesia (PCA). Compliance with ordering of multimodal analgesia and standardized anesthesia care were recorded. Patient satisfaction and QOL were measured in consenting patients.

Results: There were no differences in mean age, gender, or surgical procedures in either pre $(n=349)$ or post-ERATS group $(n=330)$. LOS was significantly shorter post ERATS (6.1 vs 4.5 days, $p<0.01$ ). There were no differences in Minor (18 vs $21.8 \%, p=0.219)$ or Major (13.8 vs $10 \%, p=0.132)$ AEs. For process outcomes, patients experienced shorter mean time to: 'outof-bed' (18.3 vs 11.7 hours, $p<0.002$ ), independent ambulation (53.9 vs $40.3 \mathrm{hrs}, \mathrm{p}<0.001)$, successful fluid intake (30.3 vs $16.4 \mathrm{hrs}, \mathrm{p}<0.013$ ), last chest tube removal (95.3 vs $76.7 \mathrm{hrs}$, $\mathrm{p}<0.034)$, and urinary catheter removal $(50.9 \mathrm{vs} 31.6 \mathrm{hrs}, \mathrm{p}<0.001)$. There was no difference in the duration of epidural (87.4 vs $62.5 \mathrm{hrs}, \mathrm{p}=0.06$ ) and PCA administration (45 vs $48.3 \mathrm{hrs}$, $p=0.209)$ as well as 30 -day ER visits ( 21.9 vs $14.5 \%, p=0.051)$ and 30 -day re-admission ( 9.5 vs $5 \%, p=0.081$ ). Following our recommendations, we noticed increased administration to appropriate patients of preoperative Pregabalin ((46.9 vs $62 \%, p<0.001)$ and intraoperative 
Dexamethasone (76 vs $85.7 \%, p<0.001)$, but no difference in preoperative Celebrex use (62.7 vs $66.2 \%, p=0.398)$, protective one lung ventilation strategy (98.2 vs $99.7 \%, p=0.062)$, or muscle relaxant reversal (95 vs $96.2 \%, p=0.414$ ). Average measures for patient satisfaction (88.4 vs 86.8 ) and QOL (42.2 vs 42.3 ) showed no difference between the two groups.

Conclusion: ERATS implementation significantly improved length of stay, expedited feeding, ambulation and tube removal, without increasing AEs or readmissions. Ongoing research will help further refine ERATS best practice.

\section{REFERENCES:}

1. Canadian Cancer Society https://www.cancer.ca/en/cancer-information/cancertype/lung/statistics/?region=on

2. Batchelor TJP et al. Guidelines for enhanced recovery after lung surgery: recommendations of the Enhanced Recovery After Surgery (ERAS®) Society and the European Society of Thoracic Surgeons (ESTS). Eur J Cardiothorac Surg. 2019 Jan 1;55(1):91-115. 


\title{
Lumbar Cerebrospinal Fluid Drain Complications in Patients Undergoing Open and Endovascular Thoracoabdominal Aortic Procedures
}

\author{
Jennifer Dundas ${ }^{1}$; Hessam H. Kashani ${ }^{1}$; Hilary P. Grocott ${ }^{1}$
}

1 Department of Anesthesiology, Perioperative and Pain Medicine, University of Manitoba, Winnipeg, MB, Canada;

Introduction: Acute or delayed paraplegia continues to be one of the most serious complications of thoracoabdominal aortic repair and is associated with significant morbidity and mortality. Although significant progress has been made over the past two decades, there is concern that the incidence of this complication may increase again due to lesser invasive repairs being undertaken in patients with significant comorbidities and more challenging aortic anatomy/pathology. One of the neuroprotective strategies employed to mitigate the risk of spinal cord injury ( $\mathrm{SCl}$ ) is lumbar cerebrospinal fluid (CSF) drainage which is employed to optimize spinal cord perfusion pressure (SCPP) and corresponding blood flow. While it is well established to reduce the incidence of $\mathrm{SCl}$, it is associated with a range of potential complications. We examined the incidence of complications of lumbar CSF drainage in patients undergoing both endovascular and open approaches for the treatment of thoracoabdominal aortic diseases as well as the incidence of $\mathrm{SCl}$ in this population at our institution.

Methods: Following Institutional Research Ethics Board approval (which waived the need for patient consent), we performed a retrospective analysis of all patients who underwent open or endovascular repair of thoracoabdominal aortic disease in our Cardiac Sciences Program from 2005 (program inception) to August 2019. Descriptive statistics were used to outline the complications of lumbar CSF drainage (primary outcome) and the incidence of SCI.

Results: Of the 189 patients undergoing thoracic aortic procedures for acute and chronic aortic syndromes (including aneurysm, dissection, and a variety of other diagnoses), 89 (47\%) had a lumbar CSF drain placed. Lumbar CSF drain complications occurred in 27 patients (30\%). Of the 41 patients who underwent open surgical repair with lumbar CSF drainage, $7(17 \%)$ experienced a complication attributable to the CSF drain, including $2(5 \%)$ with an intracranial hemorrhage $(\mathrm{ICH})$. Of the 48 patients who underwent endovascular repair with lumbar CSF drainage, 20 (42\%) experienced a complication attributable to CSF drainage including 2 (4\%) patients with an $\mathrm{ICH}$. Five (6\%) patients died from causes attributable to the CSF drainage. Spinal cord ischemia occurred in 22 patients (12\%).

Conclusions: Lumbar CSF drainage is associated with serious complications. Our reported rates for each individual complication are consistent with those published in the current literature. However, our overall complication rate appears higher than that previously published, in part due to differences in definition of the various complications and completeness of reporting. Our institutional incidence of $\mathrm{SCl}$ appears consistent with that published in current literature. 


\section{Multi-View 3D TEE Volume Compounding for Mitral Valve Procedure Planning}

Patrick Carnahan $^{1,2}$; John Moore ${ }^{1}$; Daniel Bainbridge ${ }^{3}$; Elvis C.S. Chen ${ }^{1,2,4}$; and Terry M. Peters ${ }^{1,2,4}$

1 Imaging Laboratories, Robarts Research Institute, London, CA,

2 School of Biomedical Engineering, Western University, London, CA

3 Department of Anesthesiology, London Health Sciences Centre ,Western University, London, CA

4 Department of Medical Biophysics, Western University, London, CA

Introduction: Current standard of care diagnostic imaging for mitral valve procedures primarily consists of transesophageal echocardiography (TEE) as it provides a clear view of the mitral valve leaflets and surrounding tissue. ${ }^{1,2}$ However, TEE has limitations in signal dropout and artefacts, particularly for structures lying below the valve such as chordae tendineae. As a result, multiple image acquisitions from multiple position along the esophagus must be made and viewed separately to observe all relevant structures. We propose a trackerless, volume compounding system to fuse mid-esophageal and transgastric 3D volumes to create a single ultrasound volume containing the mitral valve and sub-valvular structures with a high level of detail. This will enable the measurement of chordae length, which is required for procedures involving the implantation of artificial chordae.

Methods: Ethics approval was obtained from the local REB. Images were acquired from 2 patients during standard pre-operative imaging sessions using Philips Epiq TEE probes. One or more mid-esophageal volumes and four or more transgastric volumes with a high degree of overlap were acquired with ECG gating. These volumes are then rigidly registered with a semisimultaneous group wise registration method $^{3}$ using the end-diastolic phase of the cardiac cycle. Following rigid alignment, for each frame in the image sequences, non-rigid registration is performed to account for slight variation in cardiac phase. The volumes are then resampled onto a common grid, and voxel-wise weighted averaging is used to generate the final output volume.

Results: We validated this image compounding approach on two excised porcine mitral valve units, a custom silicone phantom, and on two patient data sets. The porcine valves and silicone phantom were imaged according to our clinical acquisition procedure. The porcine valves were also stained with iodine and imaged with a CT scanner to provide ground truth data. The ultrasound images were then compounded using the described approach. The compounded volumes visually replicate the structures visible in the ground truth CT scan, and chordae measurements have an error of $0.6 \pm 0.5 \mathrm{~mm}$ between modalities. Results of ultrasound compounding on patient data sets show clear delineation of the mitral valve leaflets, chordae tendineae, and papillary muscles.

Conclusions: We have described a workflow for capturing a series of images using a TEE probe during standard diagnostic imaging that can then be registered and compounded together. These compounded volumes capture the sub-valvular structures of interest for cardiac procedure planning. Capturing the necessary additional volumes can be done with only an additional ten minutes to the current standard of care diagnostic images with no modifcation of equipment. This method is able to provide clinicians with a single volume that captures the 
mitral valve and the sub-valvular structures, which will enhance the mitral valve procedure planning process.

\section{REFERENCES:}

1. Shah, P. M, Journal of Cardiology 56, 125-133 (2010).

2. Linden, A et al., The Thoracic and Cardiovascular Surgeon 65, S213-S216 (2017).

3. Wachinger, C. et al., Medical Image Computing and Computer-Assisted Intervention MICCAI 2007 327-335 (2007).
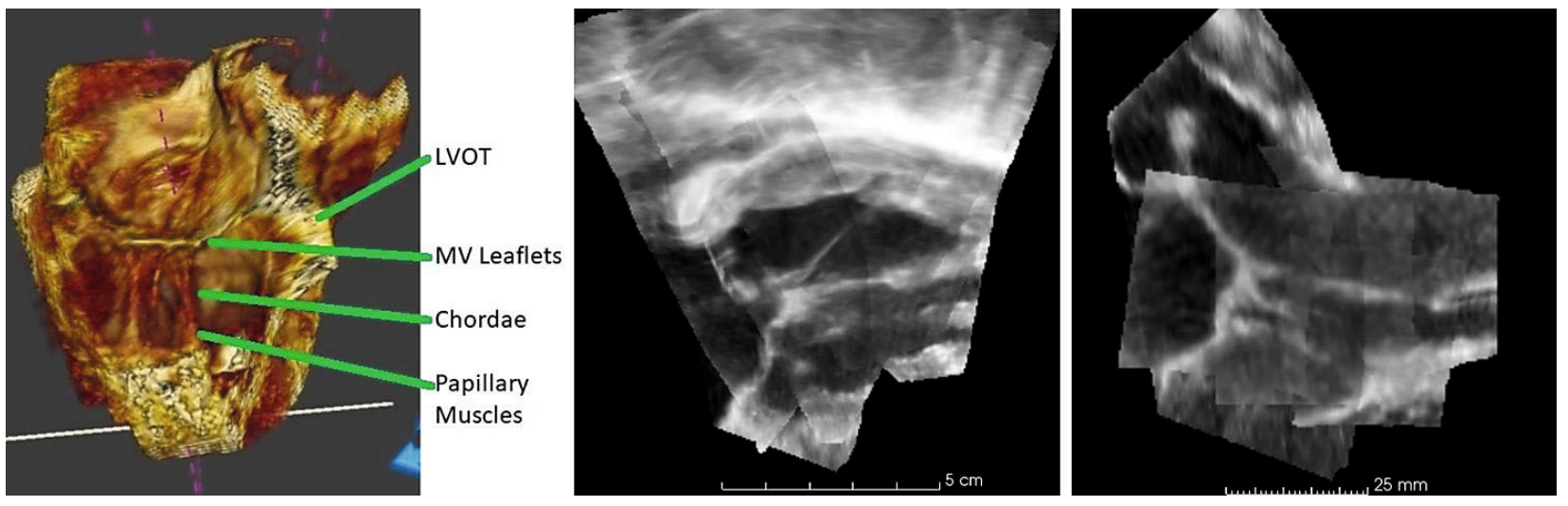


\section{Non-Invasive Monitoring of CBF and Brain Oxidative Metabolism During Cardiopulmonary Bypass: A Novel Feasibility Study}

John M Murkin ${ }^{1}$; Jason Chui ${ }^{1}$; Ajay Rajaram ${ }^{2,3}$; Daniel Milej ${ }^{2,3}$; Marianne Suwalski ${ }^{2,3}$; Lawrence Yip ${ }^{2,3}$; Michael W. A. Chu MD ${ }^{4}$; L. Ray Guo ${ }^{4}$; JohnPaul Morriseau ${ }^{5}$; Mamadou Diop PhD ${ }^{2,3}$; Keith St Lawrence ${ }^{2,3}$

1 Dept of Anesthesiology, University Hospital, Schulich School of Medicine, UWO, London, Canada;

2 Dept of Medical Biophysics, Schulich School of Medicine, UWO, London, Canada; 3Lawson Health Research Institute, London, Canada; 4Dept of Surgery, University Hospital, Schulich School of Medicine, UWO, London, Canada; 5 Clinical Perfusion Services, University Hospital, London, Canada

Introduction: Clinical management of brain-at-risk (BAR) patients is compromised by an inability to directly and non-invasively monitor cerebral blood flow (CBF) and oxidative metabolism (cytochrome $\mathrm{C}$ oxidase - $\mathrm{CCO}$ ) continuously and non-invasively during surgery.[1] As the final common pathway for oxidative metabolism, changes in $\mathrm{CCO}$ represent the intersection of perfusion pressure, flow rate, oxygen content, and metabolic demand and reflect the adequacy or compromise of substrate delivery necessary to preserve neuronal function and brain tissue viability.[2] Advances in optical techniques have enabled real-time simultaneous monitoring of CBF and CCO by combining diffuse correlation spectroscopy (DCS) and broadband near-infrared spectroscopy (bNIRS).[3] This study is the first to assess the feasibility of employing these technologies in adult BAR patients during cardiopulmonary bypass (CPB).

Methods: Ethics approval was obtained from the local REB (\#113650). 10 adult patients undergoing cardiac surgery with CPB were monitored intraoperatively with a custom built and newly modified DCS/bNIRS device[3] and hemodynamic and other biometric data were correlated to assess the impact of changes in CPB perfusion characteristics with changes in CBF and CCO.

Results: In all 10 patients, reliable signals from DCS and bNIRS device were obtained and were sufficient to demonstrate that decreases in perfusion pressure, perfusion flow rates and hemoglobin concentration had a variable impact on both CBF and CCO (see Figure). This study demonstrated diverse requirements for perfusion pressure and substrate delivery that varied between patients and within individual patients at differing times during surgery.

Conclusion: To our knowledge this study is the first such demonstration of the feasibility of using non-invasive optical devices to monitor changes in $\mathrm{CBF}$ and $\mathrm{CCO}$ in real time during $\mathrm{CPB}$ in BAR patients. It demonstrated variability in brain perfusion characteristics necessary to preserve $\mathrm{CCO}$ that differed between patients and over time in individual patients. This is further evidence of the importance of such individualized patient monitoring and represents an important first step in deploying such technology in various other high risk clinical situations. 


\section{REFERENCES:}

1. Murkin et al. Eur J Cardiothorac Surg. 2013 Jun;43(6):1081-3.

2. Bale et al., J. Biomed. Opt. 2016;21(9):099801

3. Rajaram et al. Biomed Opt Express 2018;9(6):2588

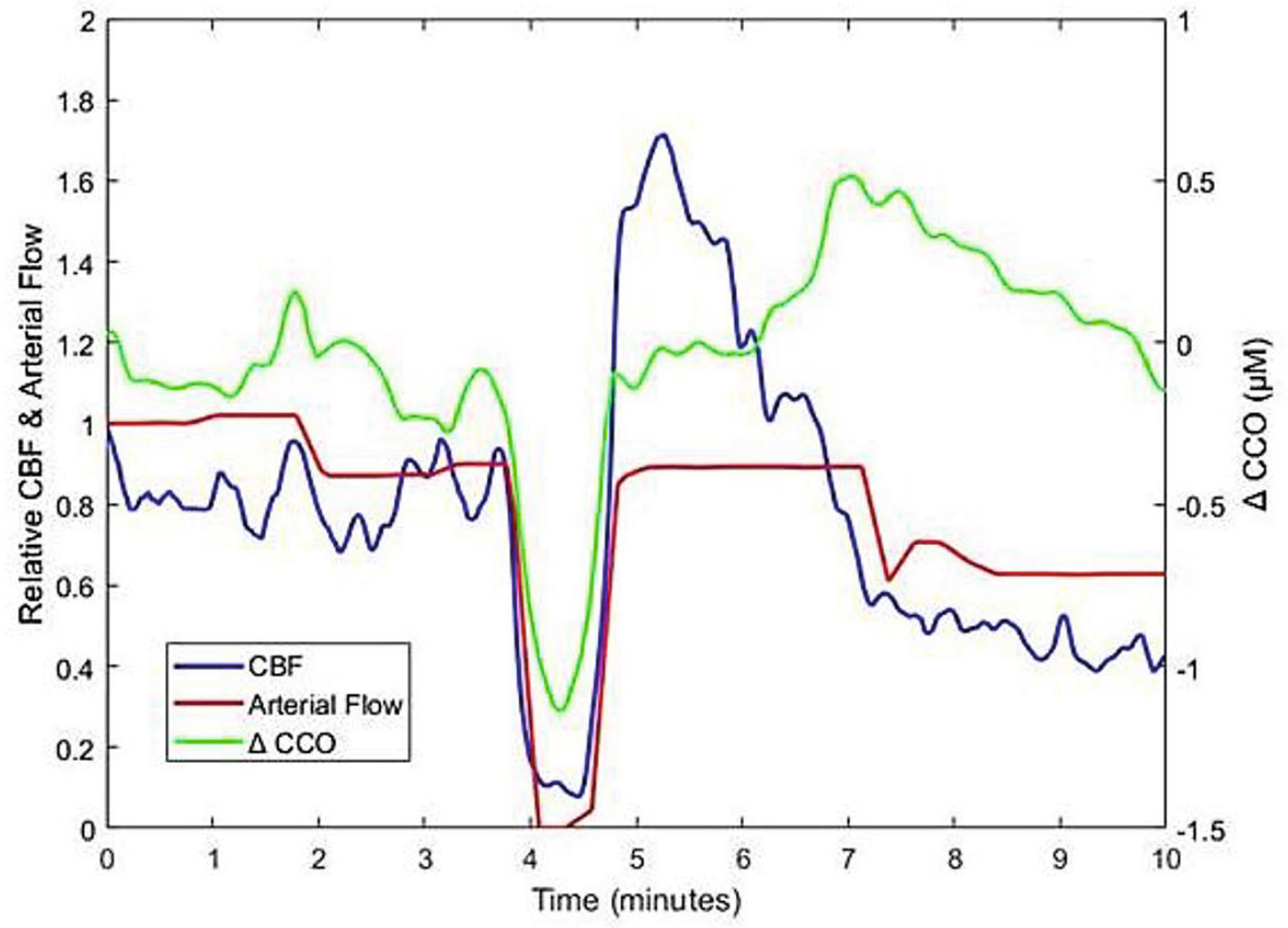

Continuous measures of CBF and metabolism ( $\triangle \mathrm{CCO}$ ) during a hypoperfusion event during cardiopulmonary bypass, as measured by DCS and bNIRS. Arterial flow rate from bypass pump shown as change from baseline. 
Rotational Thromboelastometry (ROTEM) Guided Use of Factor Concentrates in Cardiac Transplant Patients: A Retrospective Study

Kimberly C. Li; Matthew Coley²; Anton Chau²; Andrew McMillan²; Hsian Chiu²; Ron Ree ${ }^{2}$

1 Faculty of Medicine, University of British Columbia, Vancouver, BC, Canada

2 Department of Anesthesia at Providence Health Care, St. Paul's Hospital, Vancouver, BC Canada

Introduction: Orthotopic heart transplantation is performed as the last-line treatment for endstage heart failure. ${ }^{1}$ Due to a scarcity of donors, it is crucial to minimize postoperative complications and optimize patient outcome in every heart transplant case. Postoperative bleeding is one of the most common complications among patients undergoing cardiac surgery, oftentimes resulting significant morbidity and mortality. ${ }^{2-4,5}$ The etiology of perioperative bleeding is multifactorial - a combination of both patient and procedural factors. While blood transfusion in the setting of cardiac surgery is often necessary, it is associated with risks and complications including transfusion-related immunomodulation, transfusion-associated circulatory overload, transfusion error, sepsis, and transmitted diseases. ${ }^{6}$ Therefore, effective hemostasis is crucial to positive patient outcomes. At our institution, our hemostatic transfusion practice has evolved from the administration of frozen plasma and cryoprecipitate given empirically or guided by standard laboratory tests (SLTs), to administering prothrombin complex concentrates (PCCs) and fibrinogen concentrate (FC) guided by rotational thromboelastometry (ROTEM). We have developed an institutional ROTEM-guided transfusion algorithm incorporating these changes to standardize concentrated hemostatic product administration.

The goal of this project is to compare ROTEM-guided administration of factor concentrates during cardiac transplantation to the administration of blood products based on empiric judgement and SLTs with respect to intraoperative hemostatic efficiency, postoperative bleeding, red blood cell (RBC) transfusion requirements, major adverse postoperative events, and length of stay.

Methods: Ethics approval was obtained from the local REB. All protocols were approved by UBC/PHCRI Research Ethics Board. Chart review was conducted for two cohorts of cardiac transplant patients, one of which received blood products empirically while the other followed a ROTEM-guided algorithm for administration of factor concentrates. Statistical analysis was performed using Mann Whitney $U$ tests or Fisher's exact tests for continuous and categorical variables, respectively. The criterion for rejection of the null hypothesis was set at $p<0.05$.

Results: ROTEM-guided transfusion of factor concentrates was able to reduce the time required for surgical hemostasis by $47.6 \%$ (163.3 vs $85.6 \mathrm{~min}, \mathrm{p}=0.0002$ ), the total units of RBCs transfused within 24 hours post-surgery by $71.1 \%$ (9 vs 2.6 units, $p=0.0032$ ) and the total amount of bleeding via chest tube drainage by $55.5 \%$ ( 2254.5 vs $1002.4 \mathrm{ml}, p=0.0073)$. There were no statistical differences between the two groups in terms of mortality or major complications. There was a statistically significant decrease in hospital length of stay (27.5 vs 14.9 days, $p=0.0482$ ).

Conclusion: In cardiac transplant surgery, ROTEM-guided transfusion of factor concentrates improves intraoperative hemostatic efficiency, reduces postoperative bleeding and RBC 
transfusion requirements, and does not appear to increase the rate of major postoperative adverse events. Larger studies are required to confirm the efficacy, safety, and benefits of this strategy.

\section{REFERENCES:}

1. Samsky, M. D., Patel, C. B., Owen, A., Schulte, P. J., Jentzer, J., Rosenberg, P. B., ... Rogers, J. G. (2013). Ten-Year Experience With Extended Criteria Cardiac Transplantation. Circulation: Heart Failure, 6(6), 1230-1238

2. Ohmes, L. B., Di Franco, A., Guy, T. S., Lau, C., Munjal, M., Debois, W., ... Gaudino, M. (2017). Incidence, risk factors, and prognostic impact of re-exploration for bleeding after cardiac surgery: A retrospective cohort study. International Journal of Surgery, 48, 166173.

3. Karkouti, K., Wijeysundera, D. N., Yau, T. M., Beattie, W. S., Abdelnaem, E., McCluskey, S. A., ... Karski, J. (2004). The independent association of massive blood loss with mortality in cardiac surgery. Transfusion, 44(10), 1453-1462.

4. Ranucci, M., Baryshnikova, E., Castelvecchio, S., Pelissero, G., \& Surgical and Clinical Outcome Research (SCORE) Group. (2013). Major bleeding, transfusions, and anemia: the deadly triad of cardiac surgery. The Annals of Thoracic Surgery, 96(2), 478-485.

5. Woodman, R. C., \& Harker, L. A. (1990). Bleeding complications associated with cardiopulmonary bypass. Blood, 76(9), 1680-1697.

6. Howard-Quijano, K., Schwarzenberger, J. C., Scovotti, J. C., Alejos, A., Ngo, J., Gornbein, J., \& Mahajan, A. (2013). Increased red blood cell transfusions are associated with worsening outcomes in pediatric heart transplant patients. Anesthesia and Analgesia, 116(6), 1295-1308. 
Strategies to Optimize Post-Operative Atrial Fibrillation: Amiodarone Prophylaxis Protocol Reduces New-Onset Atrial Fibrillation in Patients Undergoing Elective Cardiac Surgery

Adam Merlo ${ }^{1,2}$, Jeffrey Perry ${ }^{3}$, Francine Heelan ${ }^{2}$, Christopher Mansour ${ }^{2,4}$, Madhuri

Thommandru ${ }^{1}$, Denyse Winch ${ }^{1}$, George A. Wells ${ }^{1,3}$, Diem T. T. Tran ${ }^{1}$

1 Division of Cardiac Anesthesiology, University of Ottawa Heart Institute (UOHI), Ottawa, ON, Canada

2 MD Program, University of Ottawa Faculty of Medicine, Ottawa, ON, Canada

3 Ottawa Hospital Research Institute, Ottawa, ON, Canada

4 Department of Medicine, The Ottawa Hospital, Ottawa, ON, Canada

Introduction: New-onset post-operative atrial fibrillation (POAF) is a relevant complication of cardiac surgery, increasing the risk of post-operative adverse events, length of hospital stay, health care costs, and mortality. Prophylactic prevention of POAF has been shown to decrease the economic burden after heart surgery. Despite this, the incidence continues to be $30 \%$. A clinical prediction score (SOLV AFib) was derived to help prioritize preventative therapy for patients with moderate to high risk of developing POAF. Parameters included: age over 70, planned valve procedure, obesity, evidence of left atrial dilation, and a history of stroke.

Methods: Ethics approval was obtained from the local REB. This prospective cohort study (Feb 2016 - Dec 2018) consisted of three phases (baseline, implementation, post-implementation) to introduce a prophylactic amiodarone protocol for patients $\geq 18$ years-old with no prior history of atrial fibrillation and undergoing elective $\mathrm{CABG}$ and/or valve surgery at a single center. Patients were screened using a SOLV AFib score threshold of $\geq 5$ for eligibility to receive a target dose $(4.8 \mathrm{~g})$ of amiodarone, either orally 6 days before surgery (standard protocol) or intravenously at the time of surgery (accelerated protocol) (Figure 1). Patients were followed in hospital from surgery until discharge to document POAF, management and major complications. Summary statistics and univariate analysis (Chi-squared test of categorical variables and Student's Ttest/ANOVA for continuous variables) with 95\%Cl's were reported for outcomes such as the incidence and burden of POAF, average dose of amiodarone given, and length of hospital stay.

Results: The number of patients included were: 1220 at baseline, 840 during implementation and 998 post-implementation. The groups did not differ significantly in demographics or risk factors for POAF. The protocol was started in 72\% (152/212) of eligible patients during initial implementation and in $62 \%(113 / 182)$ after $(95 \% \mathrm{Cl} 0.71 \%-19.15 \%, \mathrm{p}<0.05)$. Preoperative loading of amiodarone reduced the incidence of POAF in patients that received protocol compared to those who were at a similar level of risk (SOLV Score $\geq 5$ ) but did not receive protocol (from $55.0 \%(71 / 129)$ to $43.0 \%$ (114/265); $95 \% \mathrm{Cl} 1.51 \%-22.17 \%, \mathrm{p}<0.05)$. The average hours spent in AF decreased from $49 \pm 3$ hours pre-protocol to $36 \pm 3$ hours during implementation and $35 \pm 3$ hours post-implementation $(p<0.005)$. Among patients on protocol, $49 \%(129 / 265)$ received at least the target dose. Patients on amiodarone prophylaxis did not have a significantly shorter length of ICU or hospital stay. 
Discussion: Amiodarone prophylaxis can be useful for decreasing the incidence of POAF in higher risk patients. There was no significant decrease in protocol compliance 9 months after the initial implementation period. The lack of difference in ICU and hospital stay between the groups is likely related to incomplete loading of the amiodarone. Early discontinuation was primarily due to concerns of low heart rate and long QTc interval.

\section{REFERENCES:}

1. Mariscalco G, Engström KG. International Journal of Cardiology. 2008 Oct 13;129(3):35462.

2. Rostagno C, La Meir M, Gelsomino S, Ghilli L, Rossi A, Carone E, Braconi L, Rosso G, Puggelli F, Mattesini A, Stefàno PL. Journal of Cardiothoracic and Vascular Anesthesia. 2010 Dec 1;24(6):952-8.

3. Tran DTT, Perry JJ, Dupuis J-Y, Elmestakawy E, Wells GA. Journal of Cardiothoracic and Vascular Anesthesia. 2015 Oct;29(5):1117-1126.

4. El-Chami MF, Kilgo P, Thourani V, Lattouf OM, Delurgio DB, Guyton RA, Leon AR, Puskas JD. Journal of the American College of Cardiology. 2010 Mar 30;55(13):1370-6.

5. Mitchell LB, Exner DV, Wyse DG, Connolly CJ, Prystai GD, Bayes AJ, Kidd WT, Kieser T, Burgess JJ, Ferland A, MacAdams CL. Jama. 2005 Dec 28;294(24):3093-100. 


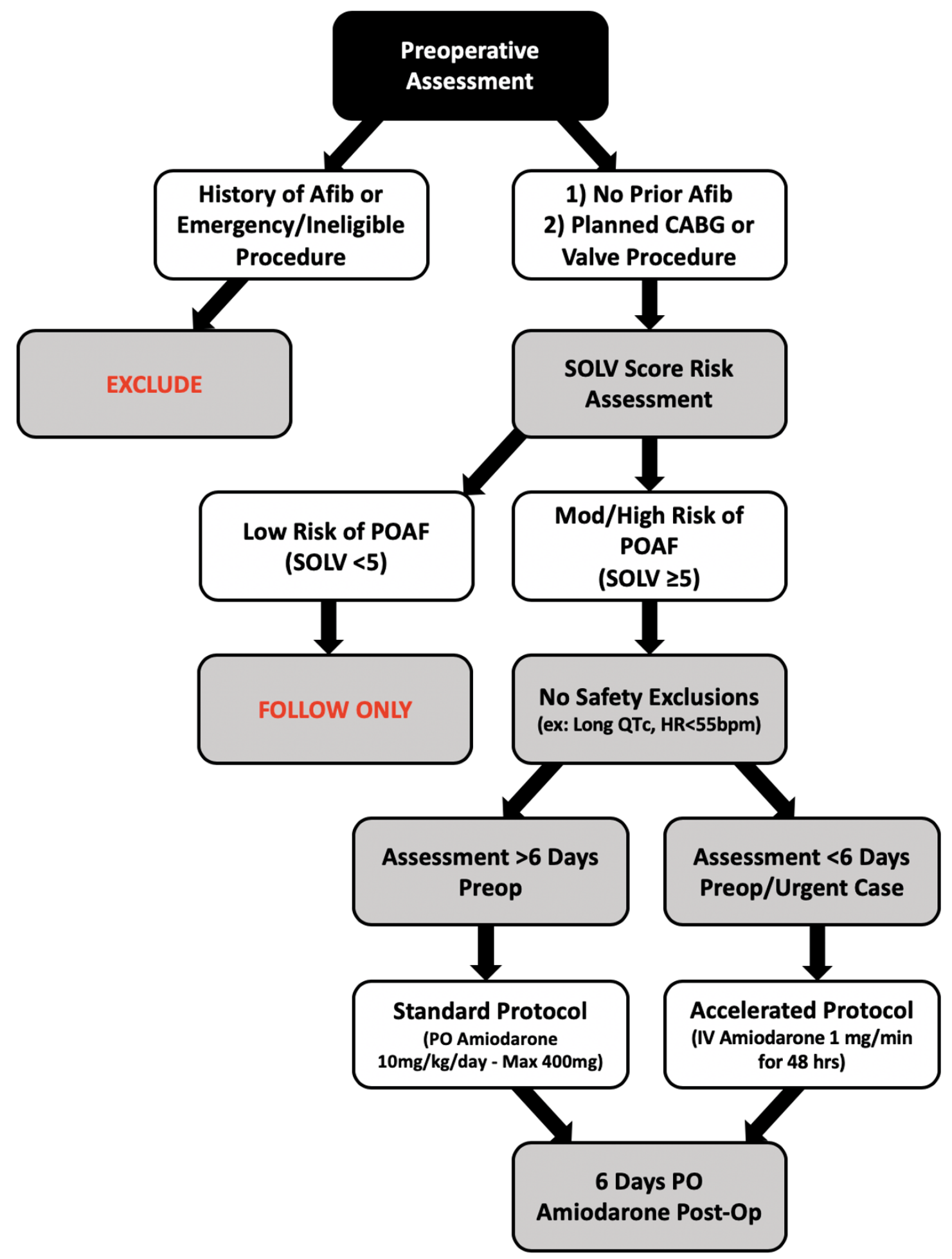




\section{CHRONIC PAIN}

\section{Adverse Events in Randomized Controlled Trials of Cannabinoids for Chronic Noncancer Pain}

Mohammed M. Mohiuddin ${ }^{1}$; Glenio B. Mizubuti ${ }^{2}$; Simon Haroutounian ${ }^{3}$; Shannon M. Smith ${ }^{4}$; Andrew S.C. Rice ${ }^{5}$; Fiona Campbell ${ }^{6}$ : Rex Park ${ }^{7}$; Ian Gilron ${ }^{8}$

1 Mohammed M. Mohiuddin, BSc., Queen's University, Anesthesiology \& Perioperative Medicine, Kingston, Ontario, Canada, mmohiuddin@qmed.ca

2 Glenio B. Mizubuti Degrees, MD, MSc, Queen's University, Anesthesiology \& Perioperative Medicine, Kingston, Ontario, Canada, glenio.mizubuti@kingstonhsc.ca

3 Simon Haroutounian, MSc, PhD, Washington University School of Medicine, Washington University Pain Centre, St. Louis, MO, USA, sharout@wustl.edu

4 Shannon M. Smith, BA, PhD, University of Rochester Medical Center, School of Medicine and Dentistry, Rochester, NY, USA, Shannon1_Smith@urmc.rochester.edu

5 Andrew S.C. Rice, MD, FRCP, FRCA FFPMRCA, FFPMCAI, Imperial College London Pain Research Group, Department of Surgery and Cancer, London, UK, a.rice@imperial.ac.uk 6 Fiona Campbell, BSc, MD, FRCA, University of Toronto, Department of Anesthesia and Pain Medicine, The Hospital for Sick Children, Toronto, ON, Canada fiona.campbell@sickkids.ca 7 Rex Park, BHSc., Queen's University, Anesthesiology \& Perioperative Medicine, Kingston, Ontario, Canada,rpark@qmed.ca

8 Ian Gilron, MD, MSc, FRCPC, Queen's University, Departments of Anesthesiology \& Perioperative Medicine, Biomedical and Molecular Sciences, Centre for Neuroscience Studies, Kingston, Ontario, Canada

Introduction: Chronic pain is a highly prevalent condition that is difficult to manage. There has been growing interest in the potential of cannabinoids for chronic pain management, with recent legalisation in both Canada and globally. The current opioid crisis highlights the need for a thorough understanding of both efficacy and safety when prescribing interventions for patients. Thus, there is a significant need to better characterize their risk-benefit profile associated with cannabinoids, particularly in the context of chronic pain.

Objective: To characterize the safety profile of cannabinoids across various intervention subtypes for the management of chronic pain.

Methods: Ethics approval was not applicable because the study did not involve human or animal research. In a recent systematic review, we searched for double-blind, randomized placebo-controlled trials (RCTs) of cannabinoids for chronic noncancer pain in MEDLINE, EMBASE, PsycINFO, CENTRAL and trial registries until June 2018. Cannabinoids administered at any dose or route were included.

Results: 43 RCTs with a total of 4436 participants with chronic pain were included. Pain conditions included central and peripheral neuropathic pain (22), spasticity in multiple sclerosis (13), chronic abdominal pain (2), chronic noncancer pain (2), fibromyalgia (2), chronic headache pain (1) and rheumatoid arthritis (1). AEs experienced in $>10 \%$ of patients and reported in $2+$ trials across intervention subtypes are reported here: smoked cannabis - dizziness, drowsiness, 
fatigue, nabiximols - asthenia, dizziness, nausea, fatigue, sublingual/vaporized cannabinoids euphoria, nabilone - drowsiness, dissociation, dry mouth, weakness, dronabinol - dizziness, headache, euphoria, dry mouth, increased appetite, weakness, drowsiness, depression, diarrhea, nausea, fatigue, oral THC/CBD capsules - asthenia, drowsiness, diarrhea, dizziness, euphoria, fatigue, weakness. Frequency of AE-related withdrawals across intervention subtypes were - sublingual spray THC:CBD (12.5\%), synthetic cannabinoids (10.0\%) nabilone (9.4\%), nabiximols $(7.5 \%)$, oral THC/CBD capsules $(7.0 \%)$, dronabinol $(6.4 \%)$, smoked cannabis $(2.3 \%)$. Frequency of SAEs across invention subtypes were dronabinol $(19.1 \%)$, oral THC/CBD capsules $(4.4 \%)$, nabiximols $(3.3 \%)$, smoked cannabis $(0 \%)$, sublingual/vaporised THC $(0 \%)$, nabilone $(0 \%)$ compared to placebo $(4.7 \%)$. Overall, all-cause withdrawals ranged from $8-17 \%$ for cannabinoid interventions compared to $0-17 \%$ for placebo.

Discussion/Conclusion: Numerous AEs were identified that could adversely impact quality of life and adherence to treatment, with different frequencies and AE profiles across intervention subtypes. The AEs experienced across 3+ subtypes included fatigue, weakness and drowsiness The greatest frequencies of $A E$ withdrawals was associated with sublingual THC:CBD spray, synthetic cannabinoids and nabilone, while the greatest frequency of SAEs was associated with dronabinol, with the remaining subtypes comparable to rates for placebo. The rates of all-cause withdrawals across intervention subtypes were comparable to placebo. These and other harms data may aid clinicians and patients in making informed decisions about cannabinoids for managing chronic pain, particularly in prescribing specific subtypes of cannabinoids for patients with certain sensitivities to AEs.

\section{REFERENCES}

1. Blyth FM, Huckel Schneider C. Global burden of pain and global pain policy-creating a purposeful body of evidence. Pain. 2018;159(suppl 1):S43-S48

2. Volkow ND, Baler RD, Compton WM, et al. Adverse health effects of marijuana use. N Engl J Med. 2014;370:2219-2227.

3. Häuser W, Finn DP, Kalso E, et al. European Pain Federation (EFIC) position paper on appropriate use of cannabis-based medicines and medical cannabis for chronic pain management. Eur J Pain. 2018;22:1547-1564 


\title{
Does the Speed of Sternal Retraction During Coronary Artery Bypass Graft Surgery Affect Postoperative Pain Outcomes?: A Randomized Controlled Trial
}

\author{
Craig Stewart $^{1,2}$; Deborah DuMerton ${ }^{1}$; Dimitri Petsikas ${ }^{3}$; Tarit Saha ${ }^{1}$
}

1 Department of Anesthesiology and Perioperative Medicine, Queen's University, Kingston, Canada

2 School of Medicine, Queen's University, Kingston, Canada

3 Department of Surgery, Queen's University, Kingston, Canada

Introduction: Chronic pain is a serious health issue impacting both the quality of life and productivity of patients. ${ }^{1-3}$ Chronic post-sternotomy pain (CPSP) is characterized by numbness, severe tenderness on palpation, allodynia, as well as constant pain across the anterior chest wall that can persist for months to years after sternotomy. ${ }^{4}$ All patients experience early postoperative pain following coronary artery bypass graft (CABG); unfortunately, approximately 30$40 \%$ of CABG patients subsequently develop CPSP. ${ }^{4-6}$

Methods: Ethics approval was obtained from the local REB. The current study is a prospective, double-blinded, randomized controlled trial. A sample size of 316 randomly assigned patients ( $n=158$ per group) was calculated to provide an $80 \%$ power at a 2 -sided $\alpha$ of 0.05 to detect a $40 \%$ decrease in CPSP incidence at 6 months. Eligible patients scheduled for elective, primary coronary artery bypass graft surgery were randomly assigned to either the CONTROL group, in which sternal retraction occurred over 30 seconds (standard practice) or the SLOW group, in which sternal retraction occurred over 15 minutes. Surgical and perioperative anesthesia protocols between the two groups were otherwise the same. Our primary outcome is the incidence of CPSP at 6 months. Secondary outcomes include: CPSP incidence at 3 and 12 months, daily chest pain intensity (NRS scale) at rest and while coughing, daily analgesic consumption, pain quality, quality of life, and pain interference with daily function at 3,6 and 12 months post-operatively.

Results: To date, 326 patients ( $n=260$ males, $n=66$ females) have taken part in the study (mean age $=65.5$ years). A total of 10 additional patients were randomized to account for participants whom did not receive the intervention, were unblinded, lost to follow-up, or expired acutely. Current rates of CPSP in our cohort are $38.2 \% / 27.3 \% / 22.5 \%$ at $3 / 6 / 12$ months follow up, respectively. The following risk factors and confounding variables for chronic poststernotomy pain were identified in our cohort: previous or active cigarette smokers $(n=86)$, type 2 diabetes mellitus $(n=146)$, atrial fibrillation $(n=20)$, obstructive sleep apnea $(n=65)$, previous myocardial infarction(s) $(n=139)$, previous coronary stenting $(n=56)$, gastroesophageal reflux disease $(n=139)$, chronic obstructive pulmonary disease $(n=23)$, previous cerebrovascular accident(s) $(n=14)$. The following serious adverse events (SAE) have been documented: prolonged post-operative ventilation ( $>24 \mathrm{hrs})(n=7)$, stroke $(n=4)$, myocardial infarction $(n=2)$, re-operation $(n=5)$, surgical bleeding (VISION criteria) $(n=14)$, sternal infection $(n=12)$, cardiac failure $(n=7)$, and death $(n=8)$.

Conclusion: The current investigation is near completion, having randomized all 326 patients. Follow-up data collection for the primary endpoint has been collected in all but 35 patients. The incidence of SAE in our cohort is consistent with previous literature in CABG surgery ${ }^{7-9}$. Furthermore, a recent interim analysis for patient safety showed no statistically significant differences in the type or rate of serious adverse events between the two study groups. 
Preliminary results will follow soon.

\section{REFERENCES:}

1. Reitsma, M. L., Vandenkerkhof, E. G., Johnston, S. L., \& Hopman, W. M. (2011). Does health-related quality of life improve in women following gynaecological surgery?. Journal of Obstetrics and Gynaecology Canada, 33(12), 1241-1247.

2. VanDenKerkhof, E. G., Hopman, W. M., Goldstein, D. H., Wilson, R. A., Towheed, T. E., Lam, M., ... \& Gilron, I. (2012). Impact of perioperative pain intensity, pain qualities, and opioid use on chronic pain after surgery: a prospective cohort study. Regional Anesthesia \& Pain Medicine, 37(1), 19-27.

3. VanDenKerkhof, E. G., Hopman, W. M., Reitsma, M. L., Goldstein, D. H., Wilson, R. A., Belliveau, P., \& Gilron, I. (2012). Chronic pain, healthcare utilization, and quality of life following gastrointestinal surgery. Canadian Journal of Anesthesia/Journal canadien d'anesthésie, 59(7), 670-680.

4. Steegers, M. A., van de Luijtgaarden, A., Noyez, L., Scheffer, G. J., \& Wilder-Smith, O. H. (2007). The role of angina pectoris in chronic pain after coronary artery bypass graft surgery. The Journal of Pain, 8(8), 667-673.

5. Cantero, C., Carolina, G., Matute, P., Tena, B., Rovira, I., \& Carmen, G. (2011). Prevalence of postoperative chronic pain after cardia surgery: 14AP2-2. European Journal of Anaesthesiology (EJA), 28, 193-194.

6. Van Gulik, L., Janssen, L. I., Ahlers, S. J., Bruins, P., Driessen, A. H., van Boven, W. J., ... \& Knibbe, C. A. (2011). Risk factors for chronic thoracic pain after cardiac surgery via sternotomy. European journal of cardio-thoracic surgery, 40(6), 1309-1313.

7. Kamel, A. T. H., Hassouna, A., El, H. E. D. A. A., \& Hikal, T. S. (2018). Major adverse cardiac events after first time elective isolated coronary artery bypass grafting: A retrospective cohort study. Journal of the Egyptian Society of Cardio-Thoracic Surgery, 26(4), 237-244. 8. Diodato, M., \& Chedrawy, E. G. (2014). Coronary artery bypass graft surgery: the past, present, and future of myocardial revascularisation. Surgery research and practice, 2014. 9. Nalysnyk, L., Fahrbach, K., Reynolds, M. W., Zhao, S. Z., \& Ross, S. (2003). Adverse events in coronary artery bypass graft (CABG) trials: a systematic review and analysis. Heart, 89(7), 767-772. 
Interactions Between Analgesic Drug Therapy and Mindfulness-Based Interventions for Chronic Pain in Adults: A Systematic Scoping Review

Rex Park'; Mohammed Mohiuddin ${ }^{1}$; Patricia Poulin ${ }^{2}$; Tim Salomons ${ }^{3,4}$; Robert Edwards ${ }^{5}$; Howard Nathan ${ }^{2}$; Chris Haley ${ }^{1}$; Ian Gilron ${ }^{1,4,6,7}$

1 Department of Anesthesiology and Perioperative Medicine, Queen's University, Kingston, Canada

2 Department of Anesthesiology and Pain Medicine, University of Ottawa, Ottawa, Canada

3 Department of Psychology, Queen's University, Kingston, Canada

4 Centre for Neuroscience Studies, Queen's University, Kingston, Canada

5 Department of Anesthesiology, Harvard University, Boston, USA

6 Department of Biomedical and Molecular Sciences, Queen's University, Kingston, Canada

7 School of Policy Studies, Queen's University, Kingston, Canada

Introduction: Chronic pain is estimated to affect 1.5 billion people worldwide and cost the United States and Canadian healthcare system over $\$ 650$ billion per year, which exceed the annual costs from cancer and heart disease. ${ }^{1-3}$ Given the well-recognized limitations of any one modality of treatment for chronic pain, the evolving concept of multimodal therapy has led to the concurrent use of 2 or more different treatment modalities for chronic pain. Since mindfulnessbased interventions (MBIs) and drug therapies likely reduce pain by different mechanisms, their combined use could provide added benefit. However, there have been no reports of interaction effects of the combination of MBls with any specific analgesic drugs. We conducted this systematic scoping review to describe the landscape of mindfulness-based trials with respect to the drug therapy and evaluate the available evidence on the interaction between MBIs and various pharmacological treatments.

Methods: Ethics approval was not applicable because the study did not involve human or animal research. We searched MEDLINE, EMBASE, CENTRAL, and PsycINFO from inception to July 2019, to identify randomized controlled trials (RCTs) that evaluated the efficacy of MBIs in chronic pain. Two authors screened all studies for inclusion and data was extracted using a form designed for this review. Primary outcomes included: (1) What concomitant analgesic drug therapies (CADTs) the trial participants were receiving; (2) If and how trials controlled for CADTs and analyzed their interaction; and (3) Results of available analyses of the interactions between $\mathrm{MBI}$ and CADTs. Secondary outcomes included frequency and severity of adverse events.

Results: Our search identified 848 citations, of which 40 RCTs were eligible for our review. $39 / 40 \mathrm{MBI}$ trials allowed participants to take CADTs, but only 15/39 of these trials provided any detail of what the CADTs were. Furthermore, only 4/39 trials controlled for CADTs the participants were receiving, and 0/39 trials analyzed the interaction between the $\mathrm{MBI}$ and CADTs. No judgment could be made about the safety of MBls because adverse events were inconsistently reported, with only 9/39 studies reporting any data regarding MBI-associated adverse events.

Discussion: A large body of evidence supports the benefits of MBIs for patients living with chronic pain. ${ }^{4,5}$ However, this review demonstrates that more trials investigating the interaction between MBIs and CADTs are needed to better define how MBIs can and should be rationally 
integrated into patients' multidisciplinary chronic pain management strategy. Additionally, with increasing interest in the potential of MBIs, there is a need to inform risk-benefit considerations. Psychological adverse events are possible during MBIs and thus better harms assessment and reporting are needed in future chronic pain mindfulness trials to characterize the safety profile of MBIs.

\section{REFERENCES:}

1. Borsook D. A future without chronic pain: neuroscience and clinical research. Cerebrum. 2012;2012:7.

2. Lynch ME. The need for a Canadian pain strategy. Pain Res Manag. 2011;16(2):77-80.

3. Gaskin DJ, Richard P. The economic costs of pain in the United States. J Pain. 2012;13(8):715-724.

4. Hilton L, Hempel S, Ewing BA, et al. Mindfulness Meditation for Chronic Pain: Systematic Review and Meta-analysis. Ann Behav Med. 2017;51(2):199-213.

5. Reiner K, Tibi L, Lipsitz JD. Do mindfulness-based interventions reduce pain intensity? A critical review of the literature. Pain Med. 2013;14(2):230-242. 


\section{Magnesium for the Management of Chronic Pain in Adults: A Systematic Review}

Rex Park ${ }^{1}$; Anthony M-H Ho ${ }^{1}$; Gisèle Pickering ${ }^{2}$; Lars Arendt-Nielsen ${ }^{3}$; Mohammed Mohiuddin ${ }^{1}$; Ian Gilron ${ }^{1,4,5}$

1 Department of Anesthesiology and Perioperative Medicine, Queen's University, Kingston, Canada

2 Department of Clinical Pharmacology, Université Clermont Auvergne, Clermont-Ferrand, France

3 Department of Health Science and Technology, Aalborg University, Aalborg, Denmark

4 Department of Biomedical and Molecular Sciences, Queen's University, Kingston, Canada

5 Centre for Neuroscience Studies, Queen's University, Kingston, Canada

Introduction: Chronic pain is a highly prevalent and complex health problem that costs the Canadian and United States healthcare system over $\$ 650$ billion per year, which exceed annual costs from cancer and heart disease. ${ }^{1,2}$ High opioid prescribing rates for chronic pain has been associated with increases in opioid-related mortality and misuse disorders, emphasizing the significant need for safer pain management strategies. ${ }^{3}$ Emerging evidence supports the safe use of magnesium in controlling chronic pain, but there is no consensus regarding its clinical effects. ${ }^{4,5}$ Thus, we conducted a systematic review to assess the current evidence of efficacy and safety of magnesium for the treatment of chronic pain.

Methods: Ethics approval was not applicable because the study did not involve human or animal research. We searched MEDLINE, EMBASE, CENTRAL, and trial registries (ICTPR and ClinicalTrials.gov) from inception to September 2018, to identify randomized controlled trials (RCTs) that evaluated the efficacy of magnesium (at any dose, frequency, or route of administration) compared to placebo in chronic pain. Participants aged 18 years and over reporting any type of chronic pain for at least 3 months were included. Primary outcomes included any participant-reported measures of pain intensity or pain relief that has been previous validated. Secondary outcomes included participants experiencing any adverse event.

Results: Our search identified 1062 citations, of which 9 RCTs containing 418 participants were eligible for our review. Three studies examined neuropathic pain (62 participants), 3 examined migraines (190 participants), 2 examined complex regional pain syndrome (86 participants), and 1 examined low back pain with a neuropathic component (80 participants). Heterogeneity of included studies precluded any meta-analyses. No judgement could be made about safety since adverse events were inconsistently reported. Evidence of analgesic efficacy from included studies was equivocal. However, reported efficacy signals in some of the included trials provide a rationale for more definitive studies. For example, there was some evidence that magnesium may provide some analgesic benefit to people with chronic low back pain with a neuropathic component following 6 weeks of magnesium treatment. ${ }^{6}$

Discussion: For the purposes of routine patient care, there is insufficient evidence to support or refute the hypothesis that magnesium is efficacious and safe in chronic pain. Larger sized trials with good assay sensitivity and better safety assessment and reporting will serve to better define the role of magnesium in the management of chronic pain. Additionally, due to differing bioavailabilities of various magnesium compounds ${ }^{7-9}$, without measuring magnesium levels, it is difficult to determine whether lack of efficacy is due to inadequate dosing or because 
magnesium is indeed not efficacious. Therefore, these future trials would ideally be stratified by baseline body magnesium levels and magnesium formulations.

\section{REFERENCES:}

1. Gaskin DJ, Richard P. The economic costs of pain in the United States. J Pain. 2012;13(8):715-724.

2. Lynch ME. The need for a Canadian pain strategy. Pain Res Manag. 2011;16(2):77-80.

3. Alford DP. Opioid Prescribing for Chronic Pain--Achieving the Right Balance through Education. N Engl J Med. 2016;374(4):301-303.

4. Nechifor M. Magnesium involvement in pain. Magnes Res. 2011;24(4):220-222.

5. Herroeder S, Schonherr ME, De Hert SG, Hollmann MW. Magnesium--essentials for anesthesiologists. Anesthesiology. 2011;114(4):971-993.

6. Yousef AA, Al-deeb AE. A double-blinded randomised controlled study of the value of sequential intravenous and oral magnesium therapy in patients with chronic low back pain with a neuropathic component. Anaesthesia. 2013;68(3):260-266.

7. Firoz M, Graber M. Bioavailability of US commercial magnesium preparations. Magnes Res. 2001;14(4):257-262.

8. Kappeler D, Heimbeck I, Herpich C, et al. Higher bioavailability of magnesium citrate as compared to magnesium oxide shown by evaluation of urinary excretion and serum levels after single-dose administration in a randomized cross-over study. BMC Nutrition. 2017;3(1):7. 9. Schuchardt JP, Hahn A. Intestinal Absorption and Factors Influencing Bioavailability of Magnesium-An Update. Curr Nutr Food Sci. 2017;13(4):260-278. 


\section{Nervous Survey II: Persistent Postsurgical Neuropathy in Female Adults after Breast} Cancer Surgery

Elvin Moynagh ${ }^{1}$; Christie Farag ${ }^{1}$; Aihua $\mathrm{Wu}^{1,2}$

1 Department of Anaesthesia and Pain Management, Maroondah Hospital, Eastern Health, VIC 3135, Australia

2 Department of Anaesthesia and Perioperative Medicine, Faculty of Medicine, Nursing and Health Sciences, Monash University, VIC 3800, Australia

Background: Recent advances in medicine have seen a significant increase in the population of long-term breast cancer survivors, highlighting the need for knowledge on late postoperative complications.

Persistent postsurgical neuropathy (PPSN), defined as the presence of a new or worsened sensorimotor deficit 3-6 months post-surgery (1), remains a large clinical problem in breast cancer survivors $(2,3)$ with potential for long term disability and psychological distress.

Aims: The primary aim of this study is to establish a baseline prevalence of PPSN in patients at 6 months or later after breast cancer surgery. The secondary aim is to examine if there is any difference in the PPSN prevalence among patients who received perioperative peripheral nerve blocks (PNB) and those who did not.

Methods: After institutional ethics approval, all adult female patients who underwent breast cancer surgery were included from 1/1/2018 to 31/12/2018.

Telephone interviews were conducted at 6 months or later after surgery. Patients were asked if they had experienced pain and/or paraesthesia in relevant nerve/plexus distribution area (surgery site and the same side of body, arm and axillary).

Results: Of the 245 eligible patients, data from 220 patients (230 breasts) were included and analyzed, among which, 50 operative breasts received PNB and 180 did not.

The median follow-up period after surgery was at 14 months (Interquartile Range 13 to 16 months).

The incidence of PPSN was 74\% (66\% for pain and $28 \%$ for paresthesia), of these, those with PNB accounted for $72 \%$ (60\% for pain and $26 \%$ for paresthesia), and without PNB $82 \%$ (67\% for pain and $29 \%$ for paresthesia).

Of concern, $28 \%$ PPSN with PNB and $26 \%$ without was significant, i.e., moderate to severe.

Conclusions: This study demonstrated that, after breast cancer surgery, PPSN remained a significant late complication in female adults and perioperative nerve blocks played limited role in reducing either the incidence or the severity of PPSN.

\section{REFERENCES:}


1. Werner MU, Kongsgaard UE. Defining persistent post-surgical pain: is an update required? BJA2014;113(1):1-4.

2. Gärtner $\mathrm{R}$, et al. Prevalence of and factors associated with persistent pain following breast cancer surgery. JAMA 2009;302(18):1985-92.

3. Mejdahl MK, et al. Persistent pain and sensory disturbances after treatment for breast cancer: six year nationwide follow-up study. BMJ 2013;346:f1865. 


\title{
CRITICAL CARE MEDICINE
}

\section{Gastric Ultrasound in Stable Patients with Decreased Level of Consciousness and Recreational Substance Use -- Are Presumed Full Stomachs Full?}

\author{
M. Brendan Munn ${ }^{1}$; Courtney Phillips ${ }^{2}$; J. Ferdows Laraya ${ }^{3}$; Gabrielle Boivin Arcouette ${ }^{4}$ \\ 1 Department of Family Medicine, University of British Columbia, Vancouver, Canada \\ 2 Department of Anesthesiology, University of Calgary, Calgary, Canada \\ 3 Department of Anesthesia, McGill University, Montreal, Canada \\ 4 Family Medicine Residency Program, University of Montreal, Montreal, Canada
}

Introduction: Intoxicated patients with decreased Glasgow Coma Scale (GCS) are common presentations to emergency departments. These patients are often intubated due to presumed full stomachs and perceived aspiration risk. Gastric ultrasound (GUS) -- a simple, non-invasive and objective option -- could be applied to this problem. This pilot study uses GUS alongside usual care at a music festival; a bounded, intoxication-dense environment where airways are often managed using non-invasive airway strategies. We aim to (1) clarify the gastric contents of any intubated patients, and (2) assess if patients managed without intubation go on to have a lack of aspiration sequelae because of empty stomachs or in spite of full stomachs.

Methods: Ethics approval was obtained from the local REB. A prospective cohort study was conducted at a multi-day music festival. Patients presenting to on-site medical services with GCS $\leq 13$ and known or suspected substance use were included. Patients with trauma, instability, metabolic derangements or additional aspiration risk factors (eg morbid obesity, pregnancy) were excluded. Standard GUS was performed by a trained provider and results were categorized according to convention as FS (full stomach, ie solids or liquids $>1.5 \mathrm{~mL} / \mathrm{kg}$ ) or ES (empty stomach, ie empty or liquids $<1.5 \mathrm{~mL} / \mathrm{kg}$ ). Additional patient data were extracted from linked medical records post event.

Results: 33 patients met inclusion criteria and 27 remained after exclusions were applied and consent obtained. 25 patients reported substance use and 19 polysubstance use. The FS group had 15 patients ( 7 solid \& 8 liquid $>1.5$ ), and the ES group had 12 patients ( 5 empty \& 12 liquid<1.5). The median low GCS documented for FS and ES was 7 and 11 respectively, and 10 patients total had a GCS of 8 or less (6 FS \& 4 ES). No patients were intubated and all were managed conservatively according to usual care. 3 patients (2 FS, $1 \mathrm{ES}$ ) were transferred to hospital. No patients re-registered at medical for clinically significant aspiration.

Conclusion: This pilot study demonstrates the potential utility of GUS in stratifying aspiration risk in intoxicated patients with decreased GCS. "Empty" stomachs might avoid intubation, while the implications and true risks of "full" stomachs for aspiration sequelae in the absence of intubation remain unclear. Due to the small numbers in this pilot study and the quoted GUS sensitivity (only 95\%), further research is needed to evaluate the safe application of this modality to clinical decision-making in intoxicated patients. 


\section{Veno-Venous ECMO for Severe Intraoperative Bronchospasm in a Pediatric Patient}

Kate Mittermaier ${ }^{1}$; Brady Warnick ${ }^{1,2}$; Cheryl Peters ${ }^{1,3}$

1 Department of Anesthesiology and Therapeutics, University of British Columbia, Vancouver, Canada

2 Department of Anesthesiology, Royal Columbian Hospital, New Westminster, Canada 3 Division of Pediatric Anesthesiology and Pediatric Critical Care, BC Children's Hospital, Vancouver, Canada

Introduction: Intraoperative bronchospasm is a well-documented complication under general anesthesia, with an overall incidence of approximately $0.2 \%$ [1]. In pediatric patients the reported incidence is between $0.3 \%-3.2 \%[2,3]$. Although rare, morbidity and mortality have been associated with severe intraoperative bronchospasm. We report a case of severe intraoperative bronchospasm in a pediatric patient, successfully salvaged by intraoperative veno-venous extra corporeal membrane oxygenation (V-V ECMO).

Case Presentation: A previously healthy 6 year old male presented to the OR for urgent reduction and fixation of a supracondylar fracture. His medical history consisted of eczema and food allergies. There was no history of reactive airway disease or hospital admissions. He had an upper respiratory tract infection approximately 2 weeks prior. He was afebrile, however had a residual non-productive cough.

Anesthetic induction with propofol and remifentanil was conducted and endotracheal intubation was performed. Subsequent to endotracheal intubation, the patient developed profound isolated bronchospasm. Treatment for bronchospasm was initiated, however the patient was refractory to comprehensive medical therapy. Over several hours a severe respiratory acidosis followed, with an arterial $\mathrm{pH}$ of 6.88 and $\mathrm{pCO} 2>110$, exceeding the upper measurable limit. There were no systemic findings consistent with anaphylaxis.

A pediatric intensive care unit (PICU) team was mobilized from the closest tertiary pediatric hospital including pediatric ECMO equipment. With worsening hemodynamics and refractory hypercapnic respiratory failure, the decision was made to place the patient on V-V ECMO. He was transported by ambulance to the PICU at the pediatric hospital on V-V ECMO. Flows were maintained at $1 \mathrm{~L} /$ minute and the patient's $\mathrm{pH}$ and pCO2 normalized. He was decannulated from ECMO less than 24 hours later and extubated the following day. 5 days after admission, the patient was discharged home from hospital without any long term sequelae. Follow up asthma and allergy testing were normal, however his nasopharyngeal culture was positive for rhinovirus.

Conclusion: A literature review was conducted on the use of V-V ECMO for rescue therapy for intraoperative bronchospasm. Two previous case reports have described successful salvage of intraoperative bronchospasm with V-V ECMO [4,5]. Both of these cases were described as bronchospasm secondary to anaphylaxis reactions. To our knowledge, this is the first case report of intraoperative salvage $\mathrm{V}-\mathrm{V}$ ECMO for isolated bronchospasm in a pediatric patient. 


\section{REFERENCES:}

1. Olsson GL. Bronchospasm during anaesthesia. A computer-aided incidence study of 136,929 patients. Acta Anaesthesiol Scand 1987; 31: 244-52.

2. von Ungern-Sternberg et al. Risk assessment for respiratory complications in paediatric anaesthesia: a prospective cohort study. Lancet. 2010 Sep;376(9743):773-83.

3. Habre et al. Incidence of severe critical events in paediatric anaesthesia (APRICOT): a prospective multicentre observational study in 261 hospitals in Europe. Lancet Respir Med. 2017;5(5):412. Epub 2017 Mar 28.

4. Scaravilli et al. ECMO for intractable status asthmaticus following atracurium. $J$ Artif Organs. 2017 Jun;20(2):178-81.

5. Kim JB, Kim JH, Song KS. Catastrophic Bronchial Spasm Due to a Severe Anaphylactic Reaction to Protamine. Korean J Thorac Cardiovasc Surg. 2016 Dec;49(6):472-4. 


\section{ECONOMICS}

\section{Reducing Acute Hospitalization Length of Stay after Total Knee Arthroplasty: A Quality Improvement Study}

Yusuke Mazda'; Yehoshua Gleicher ${ }^{1}$; Naveed Siddiquii ; Yeshith Rai ${ }^{2}$; John Matelski ${ }^{3}$; David Backstein $^{4}$, Jesse Wolfstadt ${ }^{4}$

1 Department of Anesthesia and Pain Management, Mount Sinai Hospital, University of Toronto 2 Faculty of Medicine, University of Toronto, Toronto, Canada

3 Biostatistics Research Unit, University Health Network, Toronto, Canada

4 Granovsky Gluskin Division of Orthopaedics, Department of Surgery, Mount Sinai Hospital, University of Toronto, Toronto, Canada

Introduction: Total knee arthroplasty (TKA) is an effective surgery to manage end-stage arthritis of the knee. For TKA, acute hospitalization length-of-stay (LOS) is the largest independent driver of costs and is frequently used as a measure of quality of care ${ }^{1,2}$. Studies investigating enhanced recovery after surgery (ERAS) programs for TKA have demonstrated excellent cost savings ${ }^{3}$ and reduced LOS $^{4}$. Such programs focus on improving pain management, minimising post-operative nausea and vomiting (PONV), early mobilization and enhancing patient education ${ }^{5}$. ERAS programs are designed to minimise variability in care and are developed in accordance with evidenced-based best practice.

Objectives: At our institution, LOS was identified as a key target for improving patient outcomes and minimising costs.

Methods: A root-cause-analysis was performed over a 12-month period by a multidisciplinary team of care providers to elucidate factors impacting patient recovery and discharge for elective TKA patients. Postoperative pain, PONV, delays to early mobilization due to bladder catheters, and lack of patient education for discharge home were identified as areas for improvement.

Four interventions were chosen as part of the ERAS bundle based on literature review and feasibility analysis.

- Placement of peri-operative adductor canal blocks in addition to the standard multimodal analgesia regime

- Administration of $0.1 \mathrm{mg} / \mathrm{kg}$ Dexamethasone perioperatively

- Avoidance of routine pre-operative Foley catheterization

- Pre-operative patient education to prepare for discharge on POD1

The primary outcome was acute hospital LOS. Secondary outcomes were percentage of patients discharged to inpatient rehabilitation, 24-hour opioid consumption, maximum Verbal Rating Scale score and presence of PONV in the first 24 hours. Implementation of the ERAS bundle was successfully achieved using educational rounds, training workshops, and continuous project team engagement with care providers.

Baseline data for our pre-intervention period was collected from January 2017 to December 2017. Data for the implementation period was collected from January 2018 to August 2019. 
Institutional research ethics board approval was granted for a retrospective chart review.

Results: The pre- and post-intervention groups included 232 and 383 patients respectively. Mean acute hospitalization LOS decreased from 2.82 days to 2.13 days for a net difference of 0.69 days per patient $(p<0.001)$. The percentage of patients discharged to inpatient rehabilitation decreased from $20.2 \%$ to $10.7 \%$ ( $p<0.001)$. For secondary outcome measures, mean 24-hour oral morphine consumption decreased from $60 \mathrm{mg}$ to $38 \mathrm{mg}$ for a net difference of $22 \mathrm{mg}(\mathrm{p}<0.001)$. 24-hour PONV rates decreased from $51.7 \%$ to $36.6 \%$. Bladder catheterization rates decreased from $97.0 \%$ to $37.3 \%(p<0.001)$. 30-day ED visits following discharge decreased from $12.9 \%$ to $7.3 \%(p=0.030)$.

Conclusion: Our findings provide a framework for individualized root cause analysis and a multifaceted, patient-centered intervention bundle with the potential to enhance patient recovery and decrease acute hospitalization LOS.

\section{REFERENCES:}

1. Lovse L, Poitras S, Dobransky J, et al. Should the Pareto Principle Be Applied as a Cost Savings Method in Hip and Knee Arthroplasty? J Arthroplasty 2019;34(12):2841-45.

2. Bowyer A, Royse $C$. The importance of postoperative quality of recovery: influences, assessment, and clinical and prognostic implications. Can J Anaesth 2016;63(2):176-83.

3. Larsen K, Hansen TB, Thomsen PB, et al. Cost-effectiveness of accelerated perioperative care and rehabilitation after total hip and knee arthroplasty. J Bone Joint Surg Am 2009;91(4):761-72.

4. Auyong DB, Allen CJ, Pahang JA, et al. Reduced Length of Hospitalization in Primary Total Knee Arthroplasty Patients Using an Updated Enhanced Recovery After Orthopedic Surgery (ERAS) Pathway. J Arthroplasty 2015;30(10):1705-9.

5. Kehlet $\mathrm{H}$, Thienpont E. Fast-track knee arthroplasty -- status and future challenges. Knee 2013;20 Suppl 1:S29-33. 
Sugammadex Usage in St. John's, NL (2016-2019)

Jeremy Pridham; Kylie Everard²

1 Discipline of Anesthesia, Faculty of Medicine, Memorial University of Newfoundland, St. John's, NL, Canada

2 Facultly of Medicine, Memorial University of Newfoundland, St. John's, NL, Canada

Introduction: Sugammadex is a newer neuromuscular blocking agent that has been available in Canada since April 2016. It is a fast acting reversal agent for the specific reversal of the neuromuscular blocking agent Rocuronium. Sugammadex has been on the drug formulary in St. John's, with unrestricted access to its use, since April 2016. We examined the usage of sugammadex over a three-year period from April 1, 2016 to March 31, 2019 at the two adult hospitals in St. John's Newfoundland.

Objectives: We wanted to ascertain the usage and cost of sugammadex being utilized in two adult hospitals. The cost per dose of sugammadex is approximately $\$ 100$, which is $10 \mathrm{X}$ the cost of the more traditional reversal agent Neostigmine/Glycopyrolate. We assessed what types and length of cases were most likely to have sugammadex used in. We also assessed different anesthesia providers for the use of sugammadex.

Methods: Ethics approval was obtained from the local REB. All general anesthesia cases that required muscle relaxation with the neuromuscular blocking agent rocuronium between April 1 , 2019 and March 31, 2019 were analyzed. These cases were collated and filtered for those that used sugammadex for reversal versus those that utilized Neostigmine. We also collected data showing which providers utilized sugammadex. Surgical cases were divided into specialty as well as length of time of the procedure ( $<1$ hour, $1-2$ hours and $>2$ hours).

Results: During the study period there were 19295 cases performed that used rocuronium and a reversal agent. Of this number 1834 cases were reversed with sugammadex (9.5\%). Yearly numbers showed a significant increase in sugammadex usage (Year $13.56 \%$, year $29.23 \%$ and Year $315.07 \%$ of total cases). The specialties that used the most sugammadex were General Surgery 586 (31\%), ENT/oral surgery 478 (25\%), Orthopedics $227(12 \%)$ and Thoracics 199 (11\%). There was no significant difference in usage when looking at the length of cases. It was found that one hospital used significantly more sugammadex than the other and that of the top 10 users, 8 were from this site. One provider was responsible for $13 \%$ of the Sugammadex usage.

Conclusion: Sugammadex is a relatively new drug used for the rapid reversal of neuromuscular blockade due to rocuronium. We found that there has been a steady increase in the use of sugammadex in St. John's resulting in its use in $9.5 \%$ of all cases requiring reversal during the study period. Its use is more prevalent at one adult site compared to the other. Some anesthetists use sugammadex significantly more than their peers. The total cost of Sugammadex used in St. John's during this study period was $\$ 197,777$ based on milligrams used. 


\section{EDUCATION AND SIMULATION IN ANESTHESIA}

\section{Assessing the Validity of Entrustment Scales Within a Program of Assessment for Anesthesiology}

Daniel G. Dubois ${ }^{1,3}$; Alexander J. Lingley ${ }^{2}$; Julie Ghatalia ${ }^{1}$; Meghan M. McConnell ${ }^{3}$

1 Department of Anesthesiology and Pain Medicine, University of Ottawa, Ottawa ON

2 Faculty of Medicine, University of Ottawa, Ottawa ON

3 Department of Innovation in Medical Innovation, University of Ottawa, Ottawa ON

Introduction: Competency-based medical education requires robust assessment in authentic clinical environments. Entrustment scales have emerged as a means of describing trainees' ability to perform competently using work-based assessment (WBA). However, psychometric properties of entrustment-based assessment are relatively unknown, particularly in anesthesiology. This study assessed the generalizability and extrapolation evidence for entrustment scales within a program of assessment during anesthesiology training.

Methods: Ethics approval was obtained from the local REB. Entrustment scores were collected during the first seven blocks of training for three resident cohorts. Entrustment scores were assessed during daily evaluations using a clinical case assessment tool (CCAT) within the preoperative, intraoperative, and postoperative setting. Internal consistency was calculated using Cronbach's alpha. Spearman's correlations measured the relationship between median entrustment scores and percentiles scores on the Anesthesia Knowledge Test (AKT)-1 and AKT-6, mean Objective Structured Clinical Examination (OSCE) scores, and rankings of performance by the Clinical Competence Committee (CCC).

Results: Analyses were derived from 2,309 CCATs from 35 residents. Internal consistency was highest for intraoperative scores (alpha=0.78), followed by postoperative (alpha=0.60) and preoperative (alpha=0.59) scores. Preoperative and postoperative scores correlated with median CCC rankings (preoperative: rho $=0.48$, p ponferroni $=0.024$; postoperative: rho $=0.47$, $p_{\text {bonferroni }}=0.03$ ) but not with AKT or OSCE scores (all $p_{\text {bonferroni }}>0.12$ ). Intraoperative scores significantly correlated with the AKT-6 (rho $=0.51$, p bonferroni $=0.012 ;$ ), mean OSCE (rho $=0.45$,

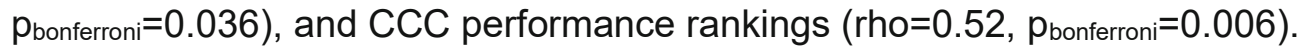

Conclusion: Within a program of assessment, the use of entrustment scales in anesthesiology training does provide early evidence of validity. Perioperative entrustment scales had good overall reliability, particularly in the intraoperative setting. Interpretation of entrustment scores in this setting may constitute a valuable adjunct to summative evaluation by the CCC. 
Assessment of CanMEDS Competencies in Work-Based Assessment: Challenges and Lessons Learned

Tul-Zahra Rida'; Daniel Dubois ${ }^{2,3}$; Yin Hui ${ }^{2}$; Julie Ghatalia ${ }^{2}$; Meghan McConnell ${ }^{2,3}$; Kori LaDonna ${ }^{3,4}$

1 Faculty of Medicine, University of Ottawa, Ottawa ON

2 Department of Anesthesiology and Pain Medicine, University of Ottawa

3 Department of Innovations in Medical Education, University of Ottawa

4 Department of Medicine, University of Ottawa

Introduction: Competency-based medical education (CBME) aims to provide an outcomes framework that clearly articulates competencies for practice. ${ }^{1,2}$ Competency-focused instruction enhanced by meaningful narrative feedback on practice competencies, elicited through workplace-based assessment (WBA), will play an essential part of CBME-based postgraduate training programs. However, success of WBA will depend on both trainees' and faculty members' ability to recognize the relevance of CanMEDS milestones in daily workplace activities, and ability to assess them through narrative WBA. ${ }^{2-6}$ Credible, actionable and timely feedback is an integral component of this process; however, literature has repeatedly shown that feedback challenges abound, particularly for non-medical expert CanMEDS intrinsic roles. ${ }^{7,8}$ This study aims to explore the distribution of narrative feedback related to milestones for the intrinsic roles, and challenges associated with their assessment in WBA.

Methods: Ethics approval for this study was obtained via the Ottawa Health Science Network Research Ethics Board. Informed consent was also obtained from study participants. In phase I of this study, a total of 3517 WBAs containing 15,834 comments were analyzed. Content analysis was used to code comments by the CanMEDS milestones framework for Anesthesiology. Descriptive statistics were then used to analyze the frequency of narrative comments for each CanMEDS role and milestone. In phase II, ten Anesthesiology residents were recruited via purposeful sampling. Residents participated in semi-structured interviews, which were informed by phase I findings, to explore challenges they experienced with CanMEDS using WBA. Interviews were anonymized and transcribed. Constructivist grounded theory informed data collection and analysis at this stage.

Results: Results from phase I demonstrated the following proportion of WBA comments per CanMEDS role: Medical Expert (36\%), Communicator (16\%), Collaborator (12\%), Scholar $(12 \%)$, Leader (8\%), Professional (8\%), and Health Advocate (8\%). However, comments were limited to a specific subset of milestones within each CanMEDS role. Strikingly, of the total 72 intrinsic role milestones, a significant proportion $(n=55,76.38 \%)$ were not adequately addressed through WBA comments. Results from phase II corroborated the aforementioned findings. Furthermore, through interviews, potential reasons were identified for why some milestones may not be addressed in WBA. These included: overlap between milestones, irrelevance of certain milestones to daily practice, need for better mapping of milestones, inappropriate conceptualization or understanding of milestones, and the aspirational nature of milestones.

Conclusion: There is evidence to suggest that not all CanMEDS roles and milestones are equally addressed in WBA. Understanding this phenomenon through ongoing data driven program evaluation would allow opportunities for targeted faculty development, revisions to the CanMEDS competency framework in the context of WBA, whilst making the framework more 
understandable and acceptable for all stakeholders. Future steps may involve structured techniques to further refine the CanMEDS framework in Anesthesiology, and determining whether this phenomenon exists within other specialties.

\section{REFERENCES:}

1. E. Stodel, A. Wyand, S. Crooks, S. Moffett, M. Chiu and C. Hudson, "Designing and Implementing a Competency-Based Training Program for Anesthesiology Residents at the University of Ottawa," Anesthesiol Res Pract, pp. 713-38, 21 Dec 2015.

2. "Competence by Design: Reshaping Canadian Medical Education [Internet]," March 2014. [Online]. Available: http://www.royalcollege.ca/rcsite/documents/cbd/competency-by-designebook-e.pdf. [Accessed 21 February 2018].

3. Holmboe, ES; Sherbino, J; Long, DM; Swing, SR; Frank, JR; ICBME Collaborators, "The role of assessment in competency-based medical education," Medical Teach, vol. 32, no. 8, pp. 676-682, 2010.

4. J. Frank, L. Snell, O. Cate, E. Holmboe, C. Carraccio, S. Swing, P. Harris, N. Glasgow, C. Campbell, D. Dath, R. Harden, W. lobst, D. Long, R. Mungroo, D. Richardson, J. Sherbino, I. Silver, S. Taber, M. Talbot and K. Harris, "Competency-based medical education: theory to practice," Med Teach, vol. 32, no. 8, p. 638-45, 2010.

5. J. Crossley, G. Humphris and B. Jolly, "Assessing health professionals," Med Educ, vol. 36, no. 9, p. 800-4, 2002.

6. Nousiainen, MT; Caverzagie, KJ; Ferguson, PC; Frank, JR; ICBME Collaborators, "Implementing competency-based medical education: What changes in curricular structure and processes are needed?," Med Teach, vol. 39, no. 6, p. 594-8, 2017.

7. N. Renting, A. Raat, T. Dornan, E. Wenger-Trayner, M. van der Wal, J. Borleffs, R. Gans and A. Jaarsma, "Integrated and implicit: how residents learn CanMEDS roles by participating in practice," Med Educ, vol. 51, no. 9, pp. 942-952, 2017.

8. G. Bandiera and D. Lendrum, "Daily encounter cards facilitate competency-based feedback while leniency bias persists," CJEM, vol. 10, no. 1, pp. 44-50, 2008. 
Coaching for Performance Change: The Development and Evaluation of a Longitudinal Academic Coaching Program for Competency-Based Medical Education Residents in Anesthesiology

Brittany Prevost $^{1}$; Lisa Bahrey ${ }^{1,2}$; Alayne Kealey ${ }^{1,3}$

1 University of Toronto Department of Anesthesiology and Pain Medicine, Toronto, ON, Canada 2 Toronto General Hospital, University Health Network, Toronto, ON, Canada

3 Sunnybrook Health Sciences Center, Toronto, ON, Canada

Introduction: Frequent observation and formative assessment with feedback is essential to a competency-based medical education (CBME) curriculum to promote trainees progressive development towards independent practice - these assessments should provide quantitative and qualitative information to enable residents to identify achievements, recognize gaps, and modify learning plans (1). Longitudinal academic coaching has been identified as one strategy to help faculty support residents and enable them to use feedback and self-reflection for their own learning progression (2).The R2C2 model, developed by Sargeant and colleagues, is an evidence-based guided reflection feedback model consisting of four steps: RELATIONSHIP building, exploring resident REACTIONS to feedback data, discussing feedback CONTENT, and COACHING for performance change (3). This model represents one framework for facilitating a coaching relationship with trainees. The purpose of this quality improvement project was to assess the utilization and initial impressions of an academic coaching program (ACP) one year after initial debut, with the intention to adjust and improve the program as it grows to include additional CBME cohorts.

Methods: Ethics approval was waived by the local REB. All participation was anonymous and voluntary. A longitudinal ACP was developed for anesthesiology CBME residents at the University of Toronto. Each resident was paired with a faculty anesthesiologist, and both were provided with information about the R2C2 framework. One year after implementation, we surveyed participants to assess the utilization of the program, perceptions, and progression through the R2C2 framework.

Results: Our survey received responses from 16 of 32 (50\%) CBME residents and 11 of 22 $(50 \%)$ faculty coaches. Pairs had met on average two times since implementation. Primary meeting topics included social connection, goals, and mentoring. Within the R2C2 model, most participants reported satisfaction with the RELATIONSHIP between trainee and coach. Many pairs had progressed to exploring REACTIONS and understanding feedback CONTENT. Fewer had reached COACHING for change, setting goals and then following up on progress. Both groups indicated a desire for increased role clarity and specific coaching strategies.

Conclusion: The survey of our ACP program highlighted satisfaction with the early stages of the R2C2 coaching framework. Based on survey responses, we have introduced changes for the current year. Near-peer discussions between CBME residents are helping to create a culture of reflection and coaching. Structure has been improved with the creation of a resident worksheet to guide pre-meeting reflection and post-meeting goal setting. Protected time for residents has helped to enable meetings or meeting preparation. The creation of a resident assessment dashboard allows residents and coaches to have complete access to resident assessment data. A follow-up survey will be repeated in spring 2020 to better understand 
interval growth in the academic coaching program and further opportunities for improvement.

\section{REFERENCES:}

1. Gofton W, Dudek N, Barton G, Bhanji F. Workplace-Based Assessment Implementation Guide: Formative tips for medical teaching practice. Ottawa: Royal College of Physicians and Surgeons of Canada; 2017.

2. Lovell B. What do we know about coaching in medical education? A literature review. Med Educ. 2018;52(4):376-90.

3. Sargeant J, Lockyer J, Mann K, Holmboe E, Silver I, Armson H, et al. Facilitated Reflective Performance Feedback: Developing an Evidence- and Theory-Based Model That Builds Relationship, Explores Reactions and Content, and Coaches for Performance Change (R2C2). Acad Med. 2015;90(12):1698-706. 
Logging out: A Comparative Analysis of Automated Logs and Resident-Driven Logbooks in Anesthesiology

Ryan McGinn ${ }^{1}$; Alexander J. Lingley²; Daniel I. Mclsaac ${ }^{1,3,4}$; Christopher Pysyk ${ }^{1}$; Meghan C. McConnell $^{5}$; Gregory L. Bryson ${ }^{1,3}$; Daniel Dubois ${ }^{1,5}$

1 Department of Anesthesiology and Pain Medicine, The Ottawa Hospital, University of Ottawa, Ottawa, ON, Canada

2 Faculty of Medicine, University of Ottawa, Ottawa, ON, Canada

3 Ottawa Hospital Research Institute, University of Ottawa, Ottawa, ON, Canada

4 School of Epidemiology and Public Health, University of Ottawa, Ottawa, ON, Canada

5 Department of Innovation in Medical Education, University of Ottawa, Ottawa, ON, Canada

Introduction: Resident logbooks of clinical case exposures are widespread in medical education despite evidence of poor accuracy and acceptance of logbook data. Electronic records (e.g. Anesthesia Information Management Systems, AIMS) may permit an objective means of auditing longitudinal case exposure. We evaluated the agreement between AIMS and Logbook for tracking case exposure during anesthesiology residency.

Methods: Ethics approval was obtained from the local REB. We performed a historical cohort study with anesthesiology residents (2011-2018, all of whom used a logbook contemporaneously with AIMS) at a multi-site academic health sciences network. The primary outcome was total case exposure; secondary outcomes were exposure for 7 surgical specialties. Correlation of case numbers between AIMS and logbook was assessed using Pearson correlation; agreement using Bland-Altman plots and intraclass correlation coefficients (ICC).

Results: Twenty-seven anesthesiology residents were included. Case numbers were greater with AIMS relative to logbook ( $649 \pm 103$ vs $583 \pm 191, p=0.049)$. Total case volumes between systems were moderately correlated $(r=0.50)$ with moderate agreement $(I C C=0.42)$. BlandAltman plots showed variable agreement between AIMS and logbook (bias = $66 \pm 166$ cases). Within the surgical specialties there was moderate agreement (ICC >0.5) for orthopedics, vascular, and neurosurgery and weaker agreement $($ ICC <0.5) for thoracics, urology, general surgery and gynecology.

Conclusion: For anesthesiology resident case logging, the number of cases logged in an AIMS was higher, with lower variance, than a logbook. The systems demonstrated moderate correlation and agreement. Given the additional time and resources required for logbooks, AIMS may be a suitable alternative for tracking case exposure where available. 


\section{Performance Assessment and Clinical Experience (PACE) Scorecards: A Post- Implementation Physician Survey}

Alexander J. Lingley ; Ryan McGinn²; Christopher Pysyk²; Gregory L. Bryson²; Daniel G. Dubois $^{2,3}$

1 Faculty of Medicine, University of Ottawa, Ottawa ON

2 Department of Anesthesiology \& Pain Medicine, University of Ottawa, Ottawa ON

3 Department of Innovations in Medical Education, University of Ottawa, ON

Introduction: Self-documented logs of clinical exposures are commonly employed to audit residents' learning experiences and infer progression towards competency. However, a recent pilot project at our institution identified marked disparity in clinical volume tracked with logbooks compared to an electronic health information system (HIS). The current study sought to characterize anesthesiology resident's perceptions of performance data extracted from a HIS specific to the perioperative setting, the anesthesia information management system (AIMS).

Methods: Ethics approval was obtained from the local REB. We collated and summarized annual process and outcomes data from AIMS. Resultant individualized performance scorecards and a previously validated 11 -item post-implementation survey were co-distributed to anesthesiology residents at a single institution $(n=42)$.

Results: We obtained a response rate of (17/42; 40\%). A majority of residents agreed/strongly agreed that scorecards were personally interesting $(15 / 17 ; 88 \%)$, valuable for professional development $(13 / 17 ; 76 \%)$, an effective measure of clinical experience $(12 / 17 ; 71 \%)$, and influential of their future practice $(12 / 17 ; 71 \%)$. A majority of residents also agreed/strongly agreed that scorecards were preferable to logbooks (12/17; 71\%). Only $41 \%(7 / 17)$ of respondents agreed/strongly agreed that scorecards were an effective measure of clinical performance. Qualitative responses indicated metrics that residents felt deserved inclusion in subsequent scorecards including anesthesia start-time as well as the number of obstetric epidurals and regional procedures performed.

Conclusion: Performance scorecards were overall well-accepted among anesthesiology residents and preferred to manual-entry logbooks. Further research is necessary to discern whether this form of feedback influences practice behaviors and/or is sensitive to clinical progression through residency. 
Simulation and Interactive Classroom in Interdisciplinary Communication Skills Training: Qualitative Analysis Reveals Strengths of Each Modality

Stephanie Power-Macdonald ${ }^{1}$; Krista Ritchie ${ }^{2,3}$; Narendra Vakharia ${ }^{1,2}$; Ana Sjaus ${ }^{1,2}$

1 Department of Anesthesiology, Pain Management and Perioperative Medicine, Dalhousie University, Halifax, Nova Scotia, Canada

2 Department of Women's and Obstetric Anesthesia, IWK Health Centre, Halifax, Nova Scotia, Canada

3 Faculty of Education, Mount Saint Vincent University, Halifax, Nova Scotia, Canada

Introduction/Background: Team communication errors contribute to poor outcomes in over $50 \%$ of sentinel cases in obstetric care. [1] Communication skill training as part of interprofessional development curricula requires careful consideration of instructional design. This study reports ethnographic descriptions of how instruction unfolds through two common teaching modalities with the same learning objectives, (1) simulation with group-debriefing and (2) guided problem-based learning (GPBL) presentation and group work. Simulation is an excellent modality for eliciting behaviours that lead to errors and modeling good communication, but how interactions evolve throughout a simulation may not predictably achieve predetermined objectives. Simulation delivery requires specialized equipment and operators. GPBL can integrate a range of key evidence-based features of good and poor communication into cases, but does not enable practice within the bounded rationality of timely complex decisions and communications in clinical care contexts.

Objectives: Deepening the understanding of how simulation and GPBL unfold with reflection on their relative instructional benefits and limitations. Evidence-based learning objectives were developed and implemented with the goal to teach communication skills to an interprofessional group of anesthesiologists, obstetricians, and nurses involved in high risk obstetric care.

Methods: Ethics approval was obtained from the local REB. Participants were assigned through stratified randomization to one of two instructional modalities. Each session was video recorded with recordings imported into MAXQDA software for coding. Task-analysis identified whether and how predetermined objectives were met and were coded for frequency of occurrence focusing on "sameness" between simulation and classroom in accordance with the study protocol. Conceptual analysis within an ethnographic framework captured the gestalt of each approach to professional development of communication skills, considering the relational nature of the participants, resources available, and social norms within the contexts.

Results: In simulation, the focus on medical management errors dominated the reflective postsimulation group discussion and participation in debriefing followed communication patterns, with dominating voices, established during simulation. Classroom sessions featured a focus group-like, in depth exploration of real-life experiences of participants as they reflected on errors of anonymous "others" and system-issues. Engagement in classroom discussion was balanced across participants. 
Conclusions: This is the first analysis of these instructional modalities in interdisciplinary settings. Both modalities provided unique and invaluable learning. While experiential learning was provided in simulation sessions, the interactive classroom sessions lead the participants to qualitatively different insights. Classroom discussions generated a greater range and richness of themes. A comprehensive program for interdisciplinary communication education should include both interactive classroom and simulation on parallel learning objectives. Such a program would have the potential to improve patient safety by holistically advancing the skills as well as addressing workplace-specific issues. Authors will share the design of integrated modalities, leaning on the relative strengths of each modality for future instructional design.

\section{REFERENCES:}

4. JCAHO. National Patient Safety Goals. 2005. www.jointcommission.org /PatientSafety/NationalPatientSafetyGoals/ 
The Perinatal Emergency Team Response Assessment (PETRA) Scale as a SelfAssessment Tool: A High-Fidelity In-Situ Simulation Study

Fergal McDonagh ${ }^{1}$; Gita Raghavan ${ }^{1}$; Aliya Nurmohamed ${ }^{1}$; Mrinalini Balki ${ }^{1}$; Sev Perelman²; Rory Windrim ${ }^{3}$

1 Department of Anesthesiology and Pain Medicine, Mount Sinai Hospital, University of Toronto, Canada

2 Department of Emergency Medicine, Mount Sinai Hospital, University of Toronto, Canada

3 Department of Obstetrics and Gynaecology, Mount Sinai Hospital, University of Toronto, Canada

Introduction: The PETRA scale was developed to assess teamwork in the management of obstetric crises. ${ }^{1}$ The scale has previously undergone testing in the simulation setting with expert evaluators and has been shown to be valid and reliable. ${ }^{2}$ However its validity and reliability for self-assessment is unknown. The aim of this study was to assess the validity and reliability of the PETRA scale as a self-assessment tool when used in an in-situ simulation environment on a high-risk labor and delivery unit.

Methods: Ethics approval was obtained from the local REB. A high-fidelity simulation of an obstetric emergency (uterine rupture) was carried out 21 times, with 6 different participants in each multi-disciplinary team. Each participant rated their team's performance using the modified PETRA scale (7 domains, 28 items) with a 5-point rubric (1=unacceptable to $5=$ perfect). Recorded simulation videos were sent to external expert raters for review. Self-assessment of team performance by team members was compared with assessments by three expert raters. The primary outcome was the PETRA scale score (overall and each of 7 domains), as assessed by individual participants and expert raters. Reliability of the PETRA scale within teams and among expert raters was analyzed using intra-class correlation (ICC). Mixed effect models were used to determine if expert ratings were statistically different from the selfassessment of teams.

Results: 125 healthcare personnel participated in the study (staff, fellows, and residents from anesthesiology and obstetrics, medical students, anesthesia assistants, and labor and delivery nurses). With the exception of one domain (situational awareness), scores on all domains and the total PETRA score were statistically different between self-raters and the expert raters, with self-raters tending to rate higher than the experts. (Total score - mean (Standard Error, SE) $4.00(0.05)$ for self-assessments, and $3.78(0.07)$ for experts' assessments) $(p=0.0001)$ (Table 1).

Low ICC showed low reliability among self-assessment teams and also among expert raters for all domains and the total score. (Total score - ICC 0.08 (95\% Cl $0.01-0.38)$ for self-assessors, and 0.09 (95\% $\mathrm{Cl} 0.00-0.77)$ for experts).

Conclusion: Our study suggests that self-assessment cannot replace expert assessment for the PETRA scale. Specifically, this study demonstrated higher rating scores with selfassessment versus assessment by external expert observers, consistent with the results of other similar simulation studies. ${ }^{3}$ 


\section{REFERENCES:}

1. Balki M et al. JOGC 2017;39:434-442

2. Balki M et al. JOGC 2017;39:523-533

3. Wieck et al. Am J Surg 2018;216:630-635

Table 1. Comparison of modified PETRA scores between self and expert assessors

\begin{tabular}{|l|l|l|l|}
\hline Domain & $\begin{array}{l}\text { Self-assessment, } \\
\text { Least Square Mean } \\
\text { (Standard error) }\end{array}$ & $\begin{array}{l}\text { Expert assessment, } \\
\text { Least Square Mean } \\
\text { (Standard error) }\end{array}$ & p-value \\
\hline Shared mental model & $4.20(0.05)$ & $3.87(0.07)$ & $<0.0001$ \\
\hline Communication & $3.81(0.06)$ & $3.65(0.08)$ & 0.0484 \\
\hline Situation awareness & $4.02(0.06)$ & $3.94(0.07)$ & 0.3118 \\
\hline Leadership & $3.94(0.08)$ & $3.70(0.09)$ & 0.0057 \\
\hline Followership & $3.99(0.05)$ & $3.76(0.07)$ & 0.0042 \\
\hline Workload management & $4.06(0.05)$ & $3.71(0.07)$ & $<0.0001$ \\
\hline Behaviours \& Attitudes & $4.33(0.05)$ & $4.02(0.07)$ & 0.0002 \\
\hline Total & $4.00(0.05)$ & $3.78(0.07)$ & 0.0011 \\
\hline
\end{tabular}




\title{
EQUIPMENT MONITORING
}

\section{Evaluation of Haemodynamic Impact of Video Laryngoscopy and Standard Laryngoscopy with Analgesia Nociceptive Monitoring}

\author{
Walid Atmani ${ }^{1} ;$ Mustapha bensghir ${ }^{1} ;$ Abdelouahed Baite $^{1}$
}

\section{Pole Anesthésie Réanimation Hôpital Militaire Med V Rabat}

Introduction: Standard laryngoscopy $(\mathrm{SL})$ is often accompanied by a hemodynamic reaction with increased heart rate and blood pressure. Various anesthetic methods are used to mitigate this hemodynamic reaction. Among these, the use of video laryngoscope $(\mathrm{VL})(1,2)$.

The objective of our study was to analyze the hemodynamic repercussions of LS and LV during intubation using the analgesia monitor (ANI) which can predict the hemodynamic response associated with intubation of healthy adults.

Methods: Ethics approval was obtained from the local REB. This was a prospective observational study conducted in the operating room of a military hospital. Included were patients were ASA I and II Physical Status undergoing orotracheal intubation for any surgery procedures. Patients with difficult intubation and/or ventilation criteria were excluded. Patients were randomized into two groups: standard laryngoscopy group (Gr: LS) and video laryngoscope group (Gr: VL).

Monitoring is ensured by 5 -electrode cardiovascular and non-invasive blood pressure measurement, before anesthetic induction, the analgesia nociceptive index (ANI) monitor was applied to each patient.

The anesthetic protocol (propofol 2,5mg/Kg, fentanyl $3 \mu / \mathrm{Kg}$ and rocuronium $0,6 \mathrm{mg} / \mathrm{Kg}$ ) was standardized for two groups. Data collected for each patient included demographic characteristics, hemodynamic variables (HR, SBP, DBP) during laryngoscopy, intubation and 5 min after: the instantaneous and average data of the ANI at the time of laryngoscopy, intubation and $5 \mathrm{~min}$ after. The conditions of intubation and other complications during the procedure were also noted.

Results: Eighty patients were included with 40 patients randomized to each group. There was no difference in the demographic characteristics of the two groups. Variations in ANI correlated with hemodynamic changes with good correlation $(P<0.001)$ with instantaneous $A N I$ after $5 \mathrm{~min}$ $44,30 \pm 11,97$ for LS group versus $55,77 \pm 7,62$ for VL one ., the increase in HR after $5 \mathrm{~min}$ ( LS: $80,47 \pm 12,41 / \mathrm{VL}: 74,77 \pm 8,54)$ and SBP (LS :121,85 $\pm 17,73$ / VL :112,12 $\pm 10,85$ ) was more significant in the LS group compared to the VL group with $(P=0.049, P=0.001)$ respectively.

Glottic visualization was better in the VL group $(P=0.032)$.

Conclusion: The analgesia monitor (ANI) is used to predict the hemodynamic response associated with intubation. In fact, it is an original study with simple monitoring that can provide better condition of intubation without hemodynamic reactions especially for patients with heart 
disease or neurosurgical patient with ICHT. The video laryngoscope improves the hemodynamic conditions of intubation. The analgesia monitor (ANI) is used to predict the hemodynamic response associated with intubation

\section{REFERENCES:}

1. Endotracheal intubation using videolaryngoscopy causes less cardiovascular response compared to classic direct laryngoscopy, in cardiac patients according a standard hospital protocol. Maassen RL, Pieters BM, Maathuis B, Serroyen J, Marcus MA, Wouters P et al. Acta Anaesthesiol Belg. 2012; 63:181-6.

2. Comparison between the Airtraq, X-Lite, and direct laryngoscopes for thyroid surgery: a randomized clinical trial. Bensghir M, Chouikh C, Bouhabba N, Fjjouji S, Kasouati J, Azendour H et al. Can J Anaesth. 2013; 60:377-84. 
Muscle Relaxation and Endotracheal Intubation: Textbook or Evidence Based? (Relaxed Study)

Lidia Guirguis ${ }^{1}$; James Jiang ${ }^{1}$; Hashan Samarasinghe ${ }^{1}$; Essam Qazag ${ }^{1}$; Amy Skiller ${ }^{1}$; Aihua $\mathrm{Wu}^{1,2}$

1 Department of Anaesthesia and Pain Management, Maroondah Hospital, Eastern Health, VIC 3135, Australia

2 Department of Anaesthesia and Perioperative Medicine, Faculty of Medicine, Nursing and Health Sciences, Monash University, VIC 3800, Australia

Background: Anaesthetists empirically perform endotracheal intubation (ETI) when the laryngeal muscles are supposed to be fully paralysed according to the pharmacodynamic and pharmacokinetic characteristics of the administered neuromuscular blockade drugs (NBDs) this is the 'textbook' standard. However, given patients' variable sensitivity to NBDs, routine monitoring through a quantitative peripheral nerve stimulator (PNS) has been necessary (1) to ascertain that the muscles are adequately relaxed, i.e., the disappearance of train-of-four count (TOFC=0), before attempting ETI.

Aims: The primary aim is to determine the time needed after a standard dose of NBDs to reach an optimal level of muscle paralysis to facilitate intubation. The secondary aim is to establish a baseline prevalence of suboptimal ETI's in routine practice.

Methods: After local ethics approval, elective surgical adults undergoing general anaesthesia were screened, consent was obtained from those who received non-depolarising NBDs to facilitate their ETI's during induction.

A PNS (TOFscan, IDMED, France) was applied at the adductor pollicis just before anaesthesia.

The initial TOF stimulation was activated at the time of tracheal intubation. Both the intubating time and NBD dosage were at the attending Anaesthetist's discretion, and were more or less based on 'textbook', i.e., 1.5 minutes after Rocuronium was administered at a dose of $0.6 \mathrm{mg} / \mathrm{kg}$ of ideal body weight (IBW), 3minutes after Atracurium $0.5 \mathrm{mg} / \mathrm{kg}$ of total body weight (TBW). From this time onwards, auto-TOF repeated every minute. Once TOFC was "0", the study was completed.

All the time-points with TOF ratios (TOFR) and TOFC were recorded and analysed. The values were averaged with a $95 \%$ confidence interval $(\mathrm{Cl})$.

Results: Two most commonly used non-depolarising NBDs, Atracurium and Rocuronium, were evaluated in this study. Data from 62 patients (47 Rocuronium and 15 Atracurium) were analysed.

The results indicated that, in practice, Rocuronium dosage $0.7 \mathrm{mg} / \mathrm{kg} \mathrm{IBW}(95 \% \mathrm{Cl} 0.69$ to 0.80$)$ was relatively larger than the standard, and also, time to perform ETI was over 2 minutes $(95 \% \mathrm{Cl} 2.0$ to 2.5$)$ rather than the standard 1.5 minutes. Despite these factors, $81 \%$ of ETI's were performed with suboptimal muscle relaxation, with an average TOFR at ETI being $40 \%$ $(95 \% \mathrm{Cl} 27 \%$ to $53 \%)$. 
Atracurium's dosage and time to ETI were in line with the 'textbook', $0.5 \mathrm{mg} / \mathrm{kg} \mathrm{ABW}(95 \% \mathrm{Cl}$ 0.50 to 0.56$)$ and 3 minutes $(95 \% \mathrm{Cl} 2.7$ to 3.3$)$ respectively, but none of the ETI's was performed at optimal condition with the average TOFR at ETI being $72 \%(95 \% \mathrm{Cl} 51 \%$ to $93 \%)$.

Rocuronium took a shorter period than Atracurium, $5(95 \% \mathrm{Cl} 4.3$ to 6.2$)$ versus 7 minutes $(95 \% \mathrm{Cl} 5.5$ to 8.3$)$ to produce optimal intubation condition, i.e., TOFC=0.

Conclusions: In conclusion, the time needed for both Rocuronium and Atracurium to produce optimal intubation condition after a standard induction dose is much longer than 'textbook' proposed. Suboptimal ETI's were prevalent in routine anaesthetic practice.

\section{REFERENCES:}

1. Eriksson LL. Evidence based practice and neuromuscular monitoring. Its time for routine quantitative assessment. Anesthesiology 2003; 98: 1037-1039. 


\section{GENDER STUDIES}

\section{Diversity of Residency Applicants and Matriculants in Medical Specialties in Canada and the United States: A Comparative Analysis}

Gianni R. Lorello ${ }^{1,2,3}$; Julie Silver ${ }^{4}$ Kristian McCarthy ${ }^{5}$; Alana M. Flexman ${ }^{6,7}$

1 University Health Network - Department of Anesthesiology and Pain Medicine, Toronto, ON, Canada

2 The University of Toronto, Department of Anesthesiology and Pain Medicine, Toronto, ON, Canada

3 The Wilson Centre, Toronto, Ontario, Canada

4 Department of Physical Medicine and Rehabilitation, Harvard Medical School, Boston, MA, USA

5 Faculty of Medicine, University of Toronto, Toronto, ON, Canada

6 Vancouver General Hospital - Department of Anesthesiology, Vancouver, BC, Canada

7 The University of British Columbia, Department of Anesthesiology, Pharmacology and

Therapeutics, Vancouver, BC, Canada

Introduction: Equity has gained traction within Medicine, especially as it pertains to women. Women and racial minorities remain under-represented in many specialties within Medicine, despite increasingly diverse medical school enrollment. ${ }^{1}$ Data is lacking comparing the gender and racial diversity of residency applicants to different specialties in the United States (US) and Canada. Our primary objective was to compare gender representation including temporal trends of applicants to the Canadian Residency Matching Services (CaRMS) and the American Association of Medical Colleges (AAMC) between different subspecialties. Our secondary objectives were to compare the gender distribution of applied vs matched CaRMS applicants, and to compare the distribution of racial minorities amongst AAMC applicants to different subspecialties.

Methods: With institutional ethical approval, we conducted a cross-sectional, retrospective analysis of aggregate CaRMS data between 1995 and 2019 and from the AAMC between 2014 and 2018. We compared the gender composition of matriculants in the United States and Canada between 2014 and 2018 by specialty. We compared gender composition of applied vs matched Canadian and international applicants to CaRMS and analyzed the racial distribution of matched residency applicants to the AAMC, both stratified by specialty. All data analysis was performed using STATA 12.0 (StataCorp, Texas, USA).

Results: We identified 49,901 matched applicants to CaRMS over the study period, of which 46,293 (93\%) were Canadian medical graduates (CMGs) and 3,608 (7\%) were international medical graduates (IMGs). CaRMS also provided data on 55,759 applicants, including those not matched to the specialty. We identified a total of 420,139 matched applicants to the AAMC between 2014 and 2018, of which 189,073 (45\%) were US medical graduates (UMGs) and 231,066 (55\%) were IMGs. Between 2014 and 2018, women made up a greater portion of the applicant pool in Canada (45.7\%) compared to the US (37.5\%), with variation by specialty. Using specialty groupings, women were most highly represented in Obstetrics and Gynecology $(84 \%, 74 \%)$ and least represented in radiology $(30 \%, 25 \%)$ in Canada and the US, respectively. 
Women had the lowest representation in the subspecialties of neurosurgery $(22.9 \%)$ in Canada, and orthopedic surgery in the US (15.8\%). Women and men had similar match rates in Canada amongst CMGs, with higher variation amongst IMGs. In the US, racial representation varied significantly by specialty with emergency medicine having the least racial diversity and psychiatry having the most.

Discussion: Representation of gender varied significantly by medical specialty amongst applicants matched through CaRMs and the AAMC, with positive trends over time identified in some, but not all, specialties in Canada. In the US, we identified significant variation in racial representation by specialty. The reasons for these disparities between specialties require further investigation and corrective strategies identified.

\section{REFERENCES:}

1. Jena AB, Khullar D, Ho O, Olenski AR, Blumenthal DM. Sex Differences in Academic Rank in US Medical Schools in 2014. JAMA 2015;314(11):1149-58.

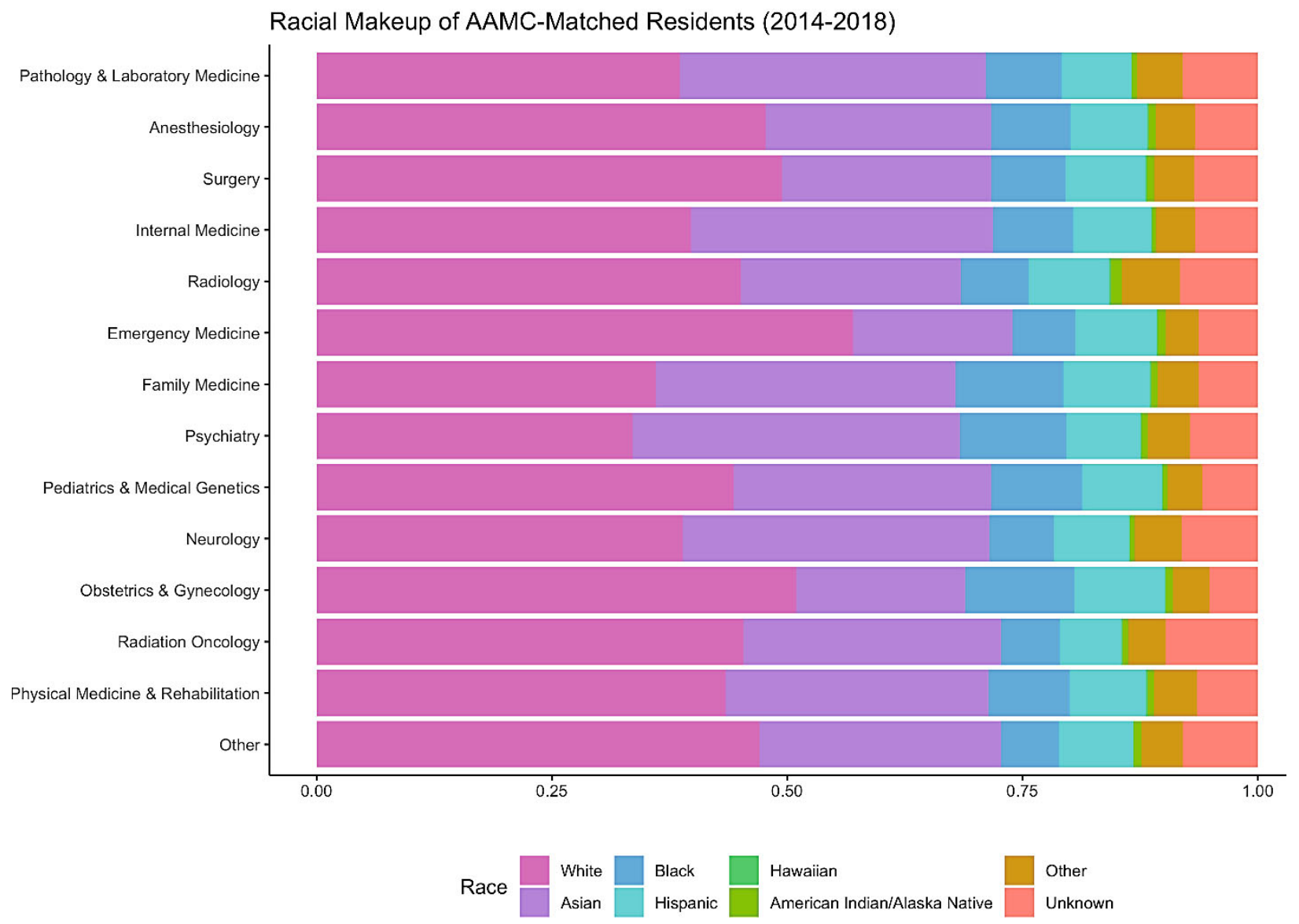


Is This a Women's World? A Retrospective Sex Analysis of Residency Applicants and Matriculants in Canadian Medical Specialties from 1995 to 2019

Gianni R. Lorello ${ }^{1,2,3}$; Julie Silver ${ }^{4}$; Kristian McCarthy ${ }^{5}$; Alana M. Flexman ${ }^{6,7}$

1 University Health Network - Department of Anesthesiology and Pain Medicine, Toronto, ON, Canada

2 The University of Toronto, Department of Anesthesiology and Pain Medicine, Toronto, ON, Canada

3 The Wilson Centre, Toronto, Ontario, Canada

4 Department of Physical Medicine and Rehabilitation, Harvard Medical School, Boston, MA, USA

5 Faculty of Medicine, University of Toronto, Toronto, ON, Canada

6 Vancouver General Hospital - Department of Anesthesiology, Vancouver, BC, Canada

7 The University of British Columbia, Department of Anesthesiology, Pharmacology and Therapeutics, Vancouver, BC, Canada

Introduction: Between 1995 to 2004, men were more likely to be rejected from their top-ranked specialty, especially in Emergency Medicine, Family Medicine, and Psychiatry. ${ }^{1}$ Gender equity has gained traction in Medicine within the last decade. Despite women comprising more than $50 \%$ of medical school classes currently, ${ }^{2}$ women remain under-represented in almost all echelons of Medicine; if the pipeline were true, the proportion of women at all levels should have increased. Therefore, our primary objectives were to compare the gender representation including temporal trends of applicants to the Canadian Residency Matching Services (CaRMS) between different subspecialties and to assess the odds of being rejected to a top-ranked specialty. Our secondary objective was to compare the gender distribution of applied vs matched CaRMS applicants.

Methods: With institutional ethical approval, we conducted a cross-sectional, retrospective analysis of aggregate data provided by CaRMS between 1995 and 2019. We compared the sex composition of matriculants in Canada by specialty, and we analyzed longitudinal trends in sex representation by specialty between 1995 and 2019 in Canada. We also analyzed the odds ratio of males and females not being matched to their top-ranked specialty. We compared sex composition of applied vs matched Canadian and international applicants to CaRMS. Specialties were condensed for portions of the analysis and to facilitate comparison. All data analysis was performed using STATA 12.0 (StataCorp, Texas, USA).

Results: We identified 49,901 matched applicants to CaRMS over the study period, of which 46,293 (93\%) were Canadian medical graduates (CMGs) and 3,608 (7\%) were international medical graduates (IMGs). CaRMS also provided data on 55,759 applicants, including those not matched to the specialty. Using specialty groupings, females were most highly represented in Obstetrics and Gynecology (84\%) and least represented in Radiology (30\%). Females had the lowest representation in the subspecialties of Neurosurgery $(22.9 \%)$. Females and males had similar match rates in Canada amongst CMGs, with higher variation amongst IMGs. However, males were more likely to be rejected from their top-ranked specialty for all disciplines, and specifically for male applicants to Family Medicine and Psychiatry; however, females were more likely to be rejected from Surgery as their top-ranked specialty. Increasing gender representation between 1995 and 2019 in Canada was seen in some, but not all, specialties. 
Discussion: Representation of sex varied significantly by medical specialty amongst applicants matched through CaRMS, with positive trends over time identified in some, but not all, specialties in Canada. Despite increasing discussions around gender equity, a trend reversal from 2005 exists where females are now more likely to be rejected from Surgery as their topranked specialty. The reasons for these disparities between specialties require further investigation and corrective strategies identified.

\section{REFERENCES:}

1. Baerlocher MO, Detsky AS. Are applicants to Canadian residency programs rejected because of their sex? CMAJ 2005;173(12):1439-40.

2. Burton KR, Wong IK. A force to contend with: The gender gap closes in Canadian medical schools. CMAJ 2004;170(9):1385-6.






\section{HEALTH MANAGEMENT}

\section{Burnout Syndrome Among Perioperative Care Providers in a Low Income Country: A Qualitative Study}

Eugene Tuyishime ${ }^{1}$; Christopher V Charles²; Lydia Yilma ${ }^{3}$; Christian Range ${ }^{4}$; Heather O'Reilly5; M. Dylan Bould ${ }^{6}$

1 OhioHealth, USA, and University of Rwanda, Kigali, Rwanda

2 Anesthesiology and Pain Management, University of Toronto, Toronto, Canada

3 Ottawa Hospital Research Institute, Ottawa, Canada

4 Department of Innovation in Medical Education, University of Ottawa, Ottawa, Canada

5 Department of Pediatric Anesthesia, Children's Hospital of Eastern Ontario, University of Ottawa, Ottawa, Canada

6 Department of Pediatric Anesthesia, Children's Hospital of Eastern Ontario, University of Ottawa, Ottawa, Canada

Introduction: Currently more than $72 \%$ of the world's population lack access to safe, timely surgical and anesthesia care. ${ }^{1}$ There is an immediate need to strengthen the global perioperative workforce, and avoiding burnout is a key part of retaining this workforce after training. ${ }^{2}$

The current study examined perioperative healthcare professionals in a low-income country (LIC), with a huge mismatch between burden of disease and numbers of perioperative healthcare providers.

Objectives: This study aimed to explore contextual issues related to job satisfaction and burnout in perioperative care providers in a LIC, and the relationship between burnout and attrition. The focus on all perioperative healthcare workers acknowledges the necessity of a well-functioning team to achieve improved outcomes in perioperative care.

Methods: Ethics approval was obtained locally and in Canada, and participant consent was collected. This data refers to the qualitative phase of a mixed-methods study. The quantitative phase will be described elsewhere. We used in-person semi-structured interviews, sampled purposively aiming to recruit participants across specialties/professions and in both rural and urban institutions across all 5 provinces.

Results: We included 55 participants, representing all hospital types (district hospital, regional referral center, teaching hospital) and perioperative professions (general practitioner, specialist physician/surgeon, midwife, non-physician anesthetist, nurse). Initial qualitative analysis suggests that key themes related to burnout and provider dissatisfaction include unpredictable work hours, poor workplace autonomy, inadequate staffing and subsequent overwhelming workload, as well as poor compensation/financial hardship. Resilience came from religious convictions, civic/national pride and belief in a slowly improving healthcare system.

Most participants indicated that if given the chance they would pursue a different profession, and that they would not encourage their family members to enter the healthcare workforce. Inadequate pay was listed as a major reason for this, and most specialist physicians and 
surgeons chose to work in private clinics to supplement their pay, despite the impact this may have on work-life balance.

Discussion: The World Health Organization estimates that by 2035 the current shortage of 7.2 million healthcare workers worldwide will increase to 12.9 million. ${ }^{3}$ Burnout is particularly common where human resources for health are few and may be a key reason for attrition. The current study demonstrates concerning features of burnout among perioperative healthcare providers in one low income country. Poor job satisfaction was noted amongst all perioperative physicians, with many suggesting that this has resulted in burnout in themselves or their colleagues. Those who can, chose to work in private clinics where they can earn more money while caring for relatively healthy (and wealthy) patients. As more healthcare providers are trained and enter the workforce, it is vital to ensure adequate retention and improved job satisfaction if this low income country is to achieve universal health coverage.

\section{REFERENCES:}

1. Meara JG, Leather AJM, Hagander L, et al. Global Surgery 2030: evidence and solutions for achieving health, welfare, and economic development. The Lancet. 2015; 386: 569-624.

2. Kudsk-Iversen S, Shamambo N, Bould MD. Strengthening the Anesthesia Workforce in Low-and Middle-Income Countries. Anesthesia and Analgesia. 2018; 126(4): 1291-1297.

3. Campbell JE. A Universal Truth: No Health Without a Workforce. Third Global Forum on Human Resources for Health Report. 2013; 104. 


\section{NEUROANESTHESIA}

\section{A Novel Approach for Assessment of the Efficacy of Somatosensory Evoked Potentials for Detection of Peripheral Nerve Injury: A Proof-of-Concept Study}

Jason Chui ${ }^{1}$; Alex Freytag ${ }^{1}$; Greydon Glimore ${ }^{2}$; Shalini Dhir ${ }^{1}$; Max Rachinsky ${ }^{1}$; John Murkin ${ }^{1}$

1 Department of Anesthesia \& Perioperative Medicine, Schulich School of Medicine \& Dentistry, University of Western Ontario, Canada

2 Department of Clinical Neurological Science, Schulich School of Medicine \& Dentistry, University of Western Ontario, Canada

Background: Somatosensory evoked potentials (SSEP) has been routinely used intraoperatively to detect nerve injury; however, the diagnostic property of SSEP has not been clearly defined in the literature (1-4). One fundamental limitation to demonstrating such a relationship is that intraoperative SSEP monitoring is used in patients under general anesthesia which precludes the possibility of real-time correlation. Another limitation is due to the rare and unpredictable occurrence of intraoperative nerve injury that requires a very large sample size study. This prospective cohort study aimed to use brachial plexus block in awake patients as an experimental model to assess the relationship between an abnormal SSEP and the symptoms of nerve dysfunction, as well as to evaluate the diagnostic values of SSEP.

Methods: Ethics approval was obtained from the local REB. Fourteen adult patients were prospectively included. We obtained baseline SSEP readings and neurological function, which was then followed by the placement of a brachial plexus block (mimicking nerve injury). We monitored the changes of SSEP and neurological symptoms simultaneously during the onset of the block to determine the temporal relationship and the diagnostic values of SSEP. Since all study participants were awake during the assessment, raw SSEP data was contaminated with motion artifacts. We custom built a graphical user interface (PySide (v1.2.4), Python (v3.6.5)) to analyse the raw data of this study. This interface allows post-hoc adjustment of the sample frequency, frequency filters, threshold and number of averaging to obtain satisfactory SSEP signals for analysis.

Results: Fourteen patients' data (170 pairs of data point) were included for final analysis. After a brachial plexus block (mimicking chemical induced nerve injury), the onset of abnormal SSEP signals almost always preceded the impairment of power $(\leq 4 / 5)$, followed by impairment of cold sensation, and of two-point discrimination (Fig. 1). The sensitivities and specificities of SSEP to detect the impairment of power $(\leq 4 / 5)$, cold sensation, and two-point discrimination were $100 \%$ and $67.4 \%, 99.1 \%$ and $54.9 \%$, and $100 \%$ and $46 \%$, respectively.

Conclusion: This is the first study that demonstrated a clear temporal relationship that abnormal SSEP almost always preceded the onset of neurological deficits. It also suggested that SSEP possesses a diagnostic property of high sensitivity and moderate specificity. Our findings suggested subcortical SSEP is a reliable screening test to detect impending intraoperative peripheral nerve injury before the injury is severe enough to become clinically apparent. This finding is highly relevant for the development of an automated SSEP nerve monitoring device for the early detection and prevention of intraoperative peripheral nerve injury 
(4-6). They also suggest that a brachial plexus block in awake patients can be used as a model for studying nerve injury to overcome a variety of methodological limitations.

\section{REFERENCES:}

1. MacDonald DB, et al: Recommendations of the International Society of Intraoperative Neurophysiology for intraoperative somatosensory evoked potentials. Clin Neurophysiol 2019; 130:161-79

2. Nuwer MR, et al: Somatosensory evoked potential spinal cord monitoring reduces neurologic deficits after scoliosis surgery: results of a large multicenter survey. Electroencephalogr Clin Neurophysiol 1995; 96:6-11

3. Ying T, et al: Clinical usefulness of somatosensory evoked potentials for detection of peripheral nerve and brachial plexus injury secondary to malpositioning in microvascular decompression. J Clin Neurophysiol 2015; 32:512-5

4. Chui J, et al: Perioperative Peripheral Nerve Injury After General Anesthesia: A Qualitative Systematic Review. Anesth Analg 2018; 127:134-43

5. Chui J, et al: A Pilot Study of a Novel Automated Somatosensory Evoked Potential (SSEP) Monitoring Device for Detection and Prevention of Intraoperative Peripheral Nerve Injury in Total Shoulder Arthroplasty Surgery. J Neurosurg Anesthesiol 2018; Publish Ahead of Print

6. Chui J, et al: A novel automated somatosensory evoked potential (SSEP) monitoring device for detection of intraoperative peripheral nerve injury in cardiac surgery: a clinical feasibility study. J Cardiothorac Vasc Anesth 2017; 31:1174-82
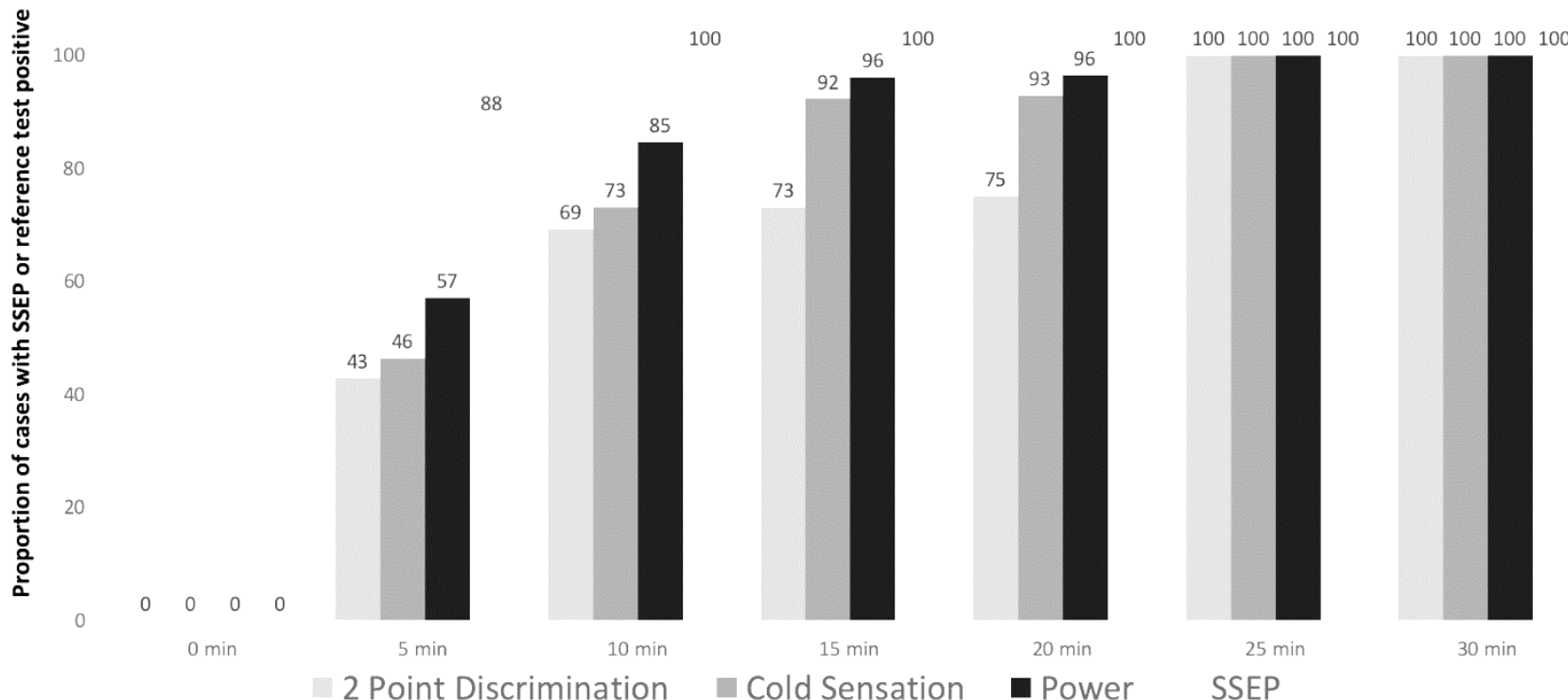

2 Point Discrimination

Cold Sensation

Power SSEP 
Anesthesia Care for Endovascular Therapy in Acute Ischaemic Stroke: A Quality Improvement Project

Luis R. Velasco Sordo ${ }^{1}$; Katherine Saied Esquivel ${ }^{1}$; Carla Todaro ${ }^{1}$; Christine C. DyValdez $^{1}$; Ruediger R. Noppens ${ }^{1}$

1 Department of Anesthesia and Perioperative Medicine, Western University/London Health Sciences Centre, London, Ontario

Introduction: Acute ischaemic stroke (AIS) for endovascular therapy is a time-critical emergency, with the number of patients who undergo this procedure currently on the rise. SNACC published an expert consensus statement in 2014 [1], however very little is known about current involvement and anesthesia care strategies in the treatment of these patients. In order to identify current anesthesiologists' approaches and potential improvements, we initiated a quality improvement (QI) project at the London Health Sciences Center in London, Ontario.

Methods: After REB approval, AIS cases that underwent endovascular therapy from March 2015 to December 2019 were retrospectively reviewed. In November 2017 a standard operational procedure (SOP) was introduced ("intervention") and the second cycle of data review was initiated covering November 2017 to December 2018. The SOP addressed an interdisciplinary management approach. A paging system, monitoring requirements, indications for anesthesia technique and physiologic targets were defined. According to SQUIRE guidelines, [2] patient characteristics, the timing of procedures, anesthesia techniques, and post-interventional transfer were evaluated. Data is presented as either an absolute number, percentage, or median (IQR). Mann-Whitney or Chi-square tests were performed where appropriate. A $p<0.05$ was considered as statistically significant.

Results: 400 patient records were analyzed (before intervention: 139, after 126). Presence of anesthesia was in 88/139 (63\%) cases before and 126/126 (100\%; $p<0.001)$ after. Time from hospital arrival until the start of anesthesia was 57 minutes (40-80), compared to 46 minutes (30-62; $p=0.002)$. General anesthesia was used in 34/139 (24\%) before, and in 25/126 (20\%; $\mathrm{p}=0.37)$ after the intervention. Total procedure time was 110 minutes (85-145) and 100 minutes (66.25-130; $p=0.03)$, respectively. After the introduction of SOP, fewer patients were transferred to ICU after endovascular therapy (19/126 (15\%) vs. 36/139 (25\%); $p=0.03)$.

Conclusion: Use of a SOP for endovascular therapy in patients with acute stroke results in a frequent involvement of anesthesia, and reduces the time until the start of intervention, total length of procedure time, and ICU admissions. The next step is to evaluate the impact on neurologic patient outcome.

\section{REFERENCES:}

1. Talke PO, Sharma D, Heyer EJ, Bergese SD, Blackham KA, Stevens RD. Society for Neuroscience in Anesthesiology and Critical Care Expert consensus statement: anesthetic management of endovascular treatment for acute ischemic stroke: endorsed by the Society of Neurolnterventional Surgery and the Neurocritical Care Society. J Neurosurg Anesthesiol. 2014;26:95-108. 
2. Davies L, Batalden P, Davidoff F, Stevens D, Ogrinc G. The SQUIRE Guidelines: an evaluation from the field, 5 years post release. BMJ Qual Saf. 2015;24:769-75. 


\section{Anesthetic Requirements and Repeat Awake Craniotomy for Tumor}

Gabriel Paquin-Lanthier ${ }^{1}$; Abigael Daniels ${ }^{1}$; Kok Weng Leong ${ }^{1}$; Lashmi Venkatraghavan ${ }^{1}$; Michael Dinsmore ${ }^{1}$; Hirokazu Takami ${ }^{2}$; Mark Bernstein ${ }^{2}$

1 Department of Anesthesiology and Pain Medicine, Toronto Western Hospital, University of Toronto, Toronto, ON, Canada

2 Department of Surgery, Division of Neurosurgery, Toronto Western Hospital, University of Toronto, Toronto, ON, Canada

Introduction: Awake craniotomy $(\mathrm{AC})$ can be a very stressful experience for patients, it has been associated with a risk of long-term psychological sequelae ${ }^{1}$. Some patients with brain tumors may need a repeat awake craniotomy $(A C)$ for a recurrence of tumor. However, there is a very limited literature on patients undergoing repeat $A C$. The aim of this study is to compare anesthesia requirements, perioperative hemodynamics, complications and outcomes between repeat $A C s$ (RAC) and the first $A C$ (FAC). We hypothesized that anesthesia and analgesia requirements and anesthesia-related complications will be less in RAC compared to the FAC.

Methods: Ethics approval was obtained from the local REB. We performed a retrospective study of all patients who had an awake craniotomy for resection of brain tumor by a single surgeon in our institution from 2010 to 2018. From the database, we identified patients who had at least two ACs during the study period, and added previous ACs to the analysis when applicable. We compared preoperative comorbidities, anesthetic drug use, hemodynamic parameters, perioperative complications and postoperative analgesia requirements between the FAC procedure and subsequent RAC. Data were analyzed using Wilcoxon signed ranked test, paired and unpaired $t$ tests as appropriate. All $p$-values are two-sided and the signification threshold is $p<0.05$.

Results: Out of 396 ACs that were performed during the study period, 28 patients had at least two ACs. After exclusion of 4 patients for missing data, 24 patients who underwent a total of 53 ACs between 2004 and 2018 were included in the final analysis; 3 patients had 3 ACs, and one patient had 4 ACs. There were no significant differences between FAC and RAC groups regarding baseline comorbidities, pre-operative and intraoperative drugs used, perioperative hemodynamic parameters and the length of surgery. However, the preoperative Karnofsky Performance Score (KPS) was lower in RAC group compared to FAC group (74.5 vs 84.5; $\mathrm{p}<0.001)$. The incidence of intraoperative mapping was also lower in RAC group $(55.2 \%$ vs $83.3 \%$ ). In analysis of paired FAC and second AC cases, the mean (SD) midazolam dose was lower in RAC compared to FAC (0.96 (0.87) mg vs 1.63 (1.56) mg; $p=0.0198)$. There were no differences between the groups with regards to perioperative complications, postoperative analgesia and antiemetic use in the recovery. Although mean (SD) oral morphine equivalents used in PACU were lower in RAC group (12.9 (13.2) mg) compared to FAC group (9.4 (11.8) $\mathrm{mg})$, this was not statistically significant.

Conclusion: Our study showed that anesthetic and analgesic requirements as well as perioperative complications were similar between first and repeat awake craniotomy. Further prospective study is needed to look at the patient satisfaction and psychological status in patients undergoing repeat awake craniotomy. 


\section{REFERENCES:}

1. Khu, K. J. et al. Patients' perceptions of awake and outpatient craniotomy for brain tumor: a qualitative study. J. Neurosurg. 112, 1056-1060 (2010). 


\section{Brain Cancer Progression: Does Anesthesia Matter? - An Initial Analysis of} Retrospective Multicenter Study

Tumul Chowdhury (Presenting Author) ${ }^{1,2}$; Kristen Gray (Med student) ${ }^{1}$; Mohit Sharma (Resident) ${ }^{1}$; Oliver Bucher ${ }^{3}$; Saranya Kakumanu ${ }^{4}$; Alberto Uribe ${ }^{5}$; Sergio D Bergese ${ }^{5,6}$; Emad Al Azazi $^{2}$; Lashmi Venkatraghavan ${ }^{2}$

1 Department of Anesthesiology, Perioperative and Pain Medicine, Health Sciences Center, University of Manitoba, Winnipeg, Canada

2 Department of Anesthesiology and Pain Medicine, Toronto Western Hospital, Toronto, Canada

3 Department of Epidemiology, Cancer Care Manitoba, Winnipeg, Canada

4 Department of Radiation Oncology, Cancer Care Manitoba, Winnipeg, Canada

5 Departments of Anesthesiology, The Ohio State University, Columbus, $\mathrm{OH}, \mathrm{USA}$

6 Department of Outcome and Research Perioperative Medicine, School of Medicine, Stony

Brook University, Stony Brook, NY, USA

Introduction: Brain tumors, depending upon type and grading, impose significant morbidity and mortality (1). Therefore, it is important to explore ways in which we can improve and prolong the lives of patients suffering from brain tumors. The role of anesthetics in this regard has yet to be explored $(2,3)$. Very few studies highlighted that volatile anesthetics have been associated with solid tumor progression whereas propofol has been shown to have a favorable effect. In addition, in some studies comparing craniotomy under sedation (awake) [AC] versus general anesthesia (GA), pain scores, plasma levels of cortisol, adrenocorticotropic hormone (ACTH), noradrenaline, and phenylalanine/tyrosine ratio were significantly higher in craniotomy under GA postoperatively (4). Thus, it is plausible that the awake craniotomy itself imposes less stress and may show less progression of the brain cancer (5). With this background, this present study will assess whether or not craniotomy without general anesthesia yields lower rates of cancer progression in patients with high-grade gliomas.

Methods: IRB approvals from all the sites were taken. This is an initial analysis of our ongoing retrospective multicenter database. All adult patients who underwent craniotomy for high-grade glioma (WHO grade III-IV) during the past ten years were included and divided into two groups: $A C$ and GA group. The primary objective was to note progression-free survival (PFS) and secondary objectives, overall survival (OS), pain scores and length of hospital stay between two groups. Data collection included patient demographics, ASA grading, WHO grading, size and location of tumor, type of anesthetics, anesthetic and surgical duration, the extent of surgical resection, histologic type, molecular diagnosis (isocitrate dehydrogenase [IDH] and O6methylguanine-DNA methyltransferase [MGMT] mutation), and postoperative adjuvant chemo/radio therapy. Progression information was retrieved using imaging and clinical signs and symptoms. Failure to return for evaluation due to death or deteriorating condition was considered to represent progression.

Results: Out of the first 500 patients, 306 patients (AC-44, GA-260) met the final inclusion criteria. Awake craniotomy cohort appears to have longer survival characteristics [both median PFS \{AC group: 0.78 (0.57-1.20)\} vs, \{GA group: 0.49 (0.44-0.55)\} and OS \{AC: $1.72(0.93-$ $2.54)\}$ vs. $\{G A: 1.04(0.85-1.28)\}$. Patients in the awake craniotomy had a greater percentage of 
patients who were IDH mutant, received more than $60 \mathrm{~Gy}$ and more than 6 cycles of chemotherapy, which might explain the longer median survival in this group of patients. However, the extent of resection, tumor volume and histological types were comparable between the two groups. Our present multicenter study, once completed, would able to answer this association more precisely.

Conclusion: This is an attempt to explore the role of minimal anesthesia $(A C)$ versus general anesthesia on high-grade glioma progression. Initial analysis favors craniotomy without general anesthesia, but not without confounding variables including genetic and adjunct radio/chemotherapy. This research will open a door for future prospective randomized trials in regards to the role of anesthetic in the brain cancer progression.

\section{REFERENCES:}

1. Louis, D.N., Ohgaki, H., Wiestler, O.D. Cavenee WK, Burger PC, Jouvet A, Scheithauer BW, Kleihues P. The 2007 WHO Classification of Tumours of the Central Nervous System. Acta Neuropathol. 2007; 114: 97-109.

2. Bharati SJ, Chowdhury T, Bergese SD, Ghosh S. Anesthetics impact on cancer recurrence: What do we know? J Can Res Ther 2016; 12: 464-8.

3. Jun IJ, Jo JY, Kim JI, Chin JH, Kim WJ, Kim HR, Lee EH, Choi IC. Impact of anesthetic agents on overall and recurrence-free survival in patients undergoing esophageal cancer surgery: A retrospective observational study. Sci Rep. 2017 Oct 25;7(1):14020. doi: 10.1038/s41598-017-14147-9.

4. Shinoura N, Yamada R, Hatori K, Sato H, Kimura K. Stress Hormone Levels in Awake Craniotomy and Craniotomy under General Anesthesia. J Neurol Neurophysiol 2014; 5: 256. doi:10.4172/2155-9562.1000256.

5. Lu VM, Phan K, Rovin RA. Comparison of operative outcomes of eloquent glioma resection performed under awake versus general anesthesia: A systematic review and meta-analysis. Clin Neurol Neurosurg. 2018; 169:121-127. 
Figure 1. Difference in PFS and $O S$ between GA and AC groups

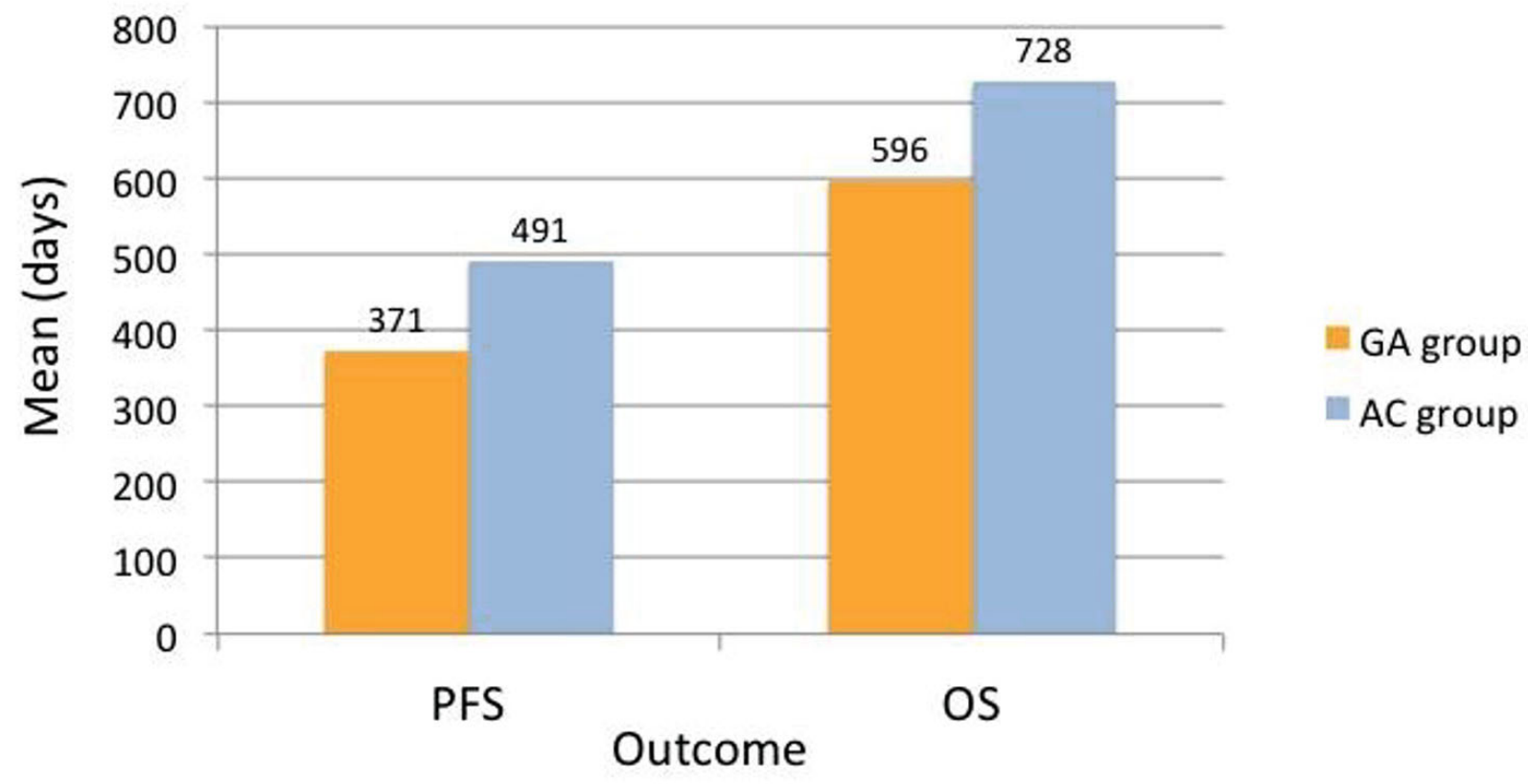




\section{Comparison of Propofol and Ketofol on Transcranial Motor Evoked Potentials (TcMEPs) in Patients Undergoing Thoracolumbar (TL) Spine Surgery}

Arvind Chaturvedi ${ }^{1}$; Ankur Khandelwal ${ }^{1}$; Navdeep Sokhal ${ }^{1}$; Akanksha Singh ${ }^{2}$; Hanjabam Barun Sharma $^{2}$

1 Department of Neuroanaesthesiology and Critical Care, AlIMS, New Delhi, India

2 Department of Physiology, AlIMS, New Delhi, India

Objectives: To compare effect of propofol and ketofol infusion (ketamine-propofol 1:4 admixture) on Motor Evoked Potential(TcMEPs), haemodynamic parameters and muscle power at discharge.

Materials and Methods: After taking Ethical approval from Institute local Etical Commettee (REB), 38 adult ASA I and II patients undergoing elective lower thoracic or Lumbar spine surgery were included i study.

Patients were randomly allocated into 2 groups $(X$ and $Y)$ in 1:1 ratio.

Amplitude and latency of Motor Evoked Potential (TcMEPs) were recorded bilaterally from Abductor Pollicis Brevis (APB) and Abductor Hallucis (AH) muscles in both upper \& lower limbs.

Baseline recordings of TcMEPs in both groups were recorded under propofol infusion. Thereafter, in group X, patients received propofol and fentanyl $1 \mathrm{mcg} / \mathrm{kg} / \mathrm{hr}$, and in group $\mathrm{Y}$, patients received ketofol and fentanyl $1 \mathrm{mcg} / \mathrm{kg} / \mathrm{hr}$. In both groups, bispectral index (BIS)was maintained between 40-60.

The amplitude and latency were recorded thereafter at 4 time points: T1 (30 mins), T2 (60 minutes), T3 (90 minutes) and T4 (120 minutes)

Results: In group X, propofol did not result in significant change in amplitude and latency in any muscle.

In group $\mathrm{Y}$, ketofol resulted in significant increase in amplitude at all time points in bilateral APB muscles and 60, 90, and 120 mins in left AH muscle without change in latency.

When the 2 groups were compared, ketofol resulted in statistically higher amplitudes at 60,90 , 120 mins in (L) APB, at 30, 60, 90, 120 mins in (R) APB and at 120 mins in both AH muscles;

latency was comparable in both groups. Blood pressures were lower whereas fluid and vasopressor requirement were higher in group $\mathrm{X}$. Muscle power was comparable between the two groups.

Conclusion: Ketofol increases amplitude of Motor Evoked Potential (TcMEPs), in comparison to Propofol, probably secondary to maintenance of haemodynamics. 


\section{REFERENCES:}

1. Kawaguchi M, Sakamoto T, Inoue S, Kakimoto M, Furuya H, Morimoto T, et al. Low dose propofol as a supplement to ketamine-based anesthesia during intraoperative monitoring of motor-evoked potentials. Spine (Phila Pa 1976). 2000; 25:974-9.

2. Zaarour C, Engelhardt T, Strantzas S, Pehora C, Lewis S, Crawford MW. Effect of low-dose ketamine on voltage requirement for transcranial electrical motor evoked potentials in children. Spine (Phila Pa 1976). 2007; 32:E627-30.

3. Furmaga H, Park HJ, Cooperrider J, Baker KB, Johnson M, Gale JT, et al. Effects of ketamine and propofol on motor evoked potentials elicited by intracranial microstimulation during deep brain stimulation. Front Syst Neurosci. 2014; 8:89. 
Intraoperative Hemodynamic Instability and Major Blood Loss during Extradural Spine Tumour Resection: A Retrospective Cohort Study

\author{
Alana Flexman ${ }^{1,2}$; Dmitry Mebel $^{2}$; Clara Kim $^{3}$; Nicolas Dea ${ }^{4}$; John Street ${ }^{4}$; Charest-Morin ${ }^{4}$ \\ 1 Department of Anesthesiology and Perioperative Care, Vancouver General Hospital, \\ Vancouver, BC, Canada \\ 2 Department of Anesthesiology, Pharmacology and Therapeutics, University of British \\ Columbia, Vancouver, BC, Canada \\ 3 Faculty of Medicine, University of British Columbia, Vancouver, BC, Canada \\ 4 Department of Orthopaedics, Division of Spine, University of British Columbia, Vancouver \\ Spine Surgery Institute, Vancouver General Hospital, Vancouver, BC, Canada
}

Background: Patients who undergo resection of extradural spine tumours undergo often highly complex surgery that is associated with a high rate of postoperative adverse events. These procedures may require staging over multiple days and, in practice, these patients experience substantial blood loss and hemodynamic instability. Few reports exist in the literature describing in detail the intraoperative anesthetic complications and postoperative disposition beyond isolated case reports.

Objectives: 1) Describe the incidence and predictors of intraoperative hemodynamic instability and major blood loss and 2) Determine the incidence of postoperative ventilation, intensive care unit (ICU) admission and length of hospital stay (LOS).

Methods: With institutional ethics approval, we identified patients who underwent extradural spine tumour resection at our institution between January 1, 2009 and October 5, 2017. We extracted demographic and patient data, anesthetic and surgical management. Our primary outcome was a composite of 1) major blood loss ( ${ }^{3} 4$ packed red blood cells or estimated blood loss $(E B L)>1000 \mathrm{ml}$ or $>1000 \mathrm{ml}$ blood salvaged/returned) and hemodynamic instability (norepinephrine infusion $>4 \mathrm{mcg} / \mathrm{min}$, phenylephrine $>0.8 \mathrm{mcg} / \mathrm{kg} / \mathrm{min}$ or systolic blood pressure $<90 \mathrm{mmHg}$ for $>10$ minutes). Secondary outcomes included postoperative ventilation, ICU admission, and LOS. Multivariate regression was used to determine independent predictors of the primary outcome. Data analysis was performed using STATA 12.1 (StataCorp, USA).

Results: We identified 101 patients and excluded 15 patients due to unavailable anesthetic records, leaving 86 patients for the analysis. Mean age was 49 (standard deviation [SD] 20) years and $58 \%(n=50)$ were male. Surgery was indicated for primary tumours in $85 \%(n=73)$ and metastatic tumours in $15 \%(n=13)$ and located in the cervical $(15 \%, n=13)$, thoracolumbar $(62 \%, n=53)$, sacral $(23 \%, n=20)$ regions. Procedures were staged in 18 patients $(21 \%)$, with an average surgical duration of 10.8 (SD 6.5) hours and EBL 2707 (SD 3711) ml. Cell salvage was used in 56 patients (65\%) and tranexamic acid in $79(92 \%)$ patients. Our primary outcome was present in 53 patients $(62 \%)(n=29$ major blood loss and $n=43$ hemodynamic instability; note $\mathrm{n}=19$ had both). Independent predictors of the primary outcome (AUROC 0.713 ) were age (adjusted OR 1.32 per decade, 95\% confidence interval [Cl] 1.02 to $1.72, p=0.035$ ) and staged procedure (adjusted OR 4.48, 95\% Cl 1.18 to 17.96, $\mathrm{p}=0.027$ ) (Figure 1). Postoperative ventilation ( $75 \%$ vs $36 \%, p<0.001)$, ICU admission $(32 \%$ vs $9 \%, p=0.018)$ and longer LOS (median 24 [IQR 16-41] vs 10 [7-16] days, $p=0.0075$ ) were more common in patients who experienced the composite outcome vs those who did not, respectively. 
Discussion: Our results quantified a high rate of intraoperative major blood loss and hemodynamic instability in patients undergoing extradural spine tumour resection. Anesthesiologists should prepare appropriately, particularly in older patients undergoing staged procedures. Postoperative ventilation, ICU admission and longer LOS should be anticipated.

\section{REFERENCES:}

1. Charest-Morin R, Flexman AM, Srinivas S, Fisher CG, Street JT, Boyd MC, et al. Perioperative adverse events following surgery for primary bone tumors of the spine and en bloc resection for metastases. J Neurosurg Spine. 2019:1-8.

2. Velanovich V, Antoine H, Swartz A, Peters D, Rubinfeld I. Accumulating deficits model of frailty and postoperative mortality and morbidity: its application to a national database. J Surg Res. 2013;183(1):104-10.

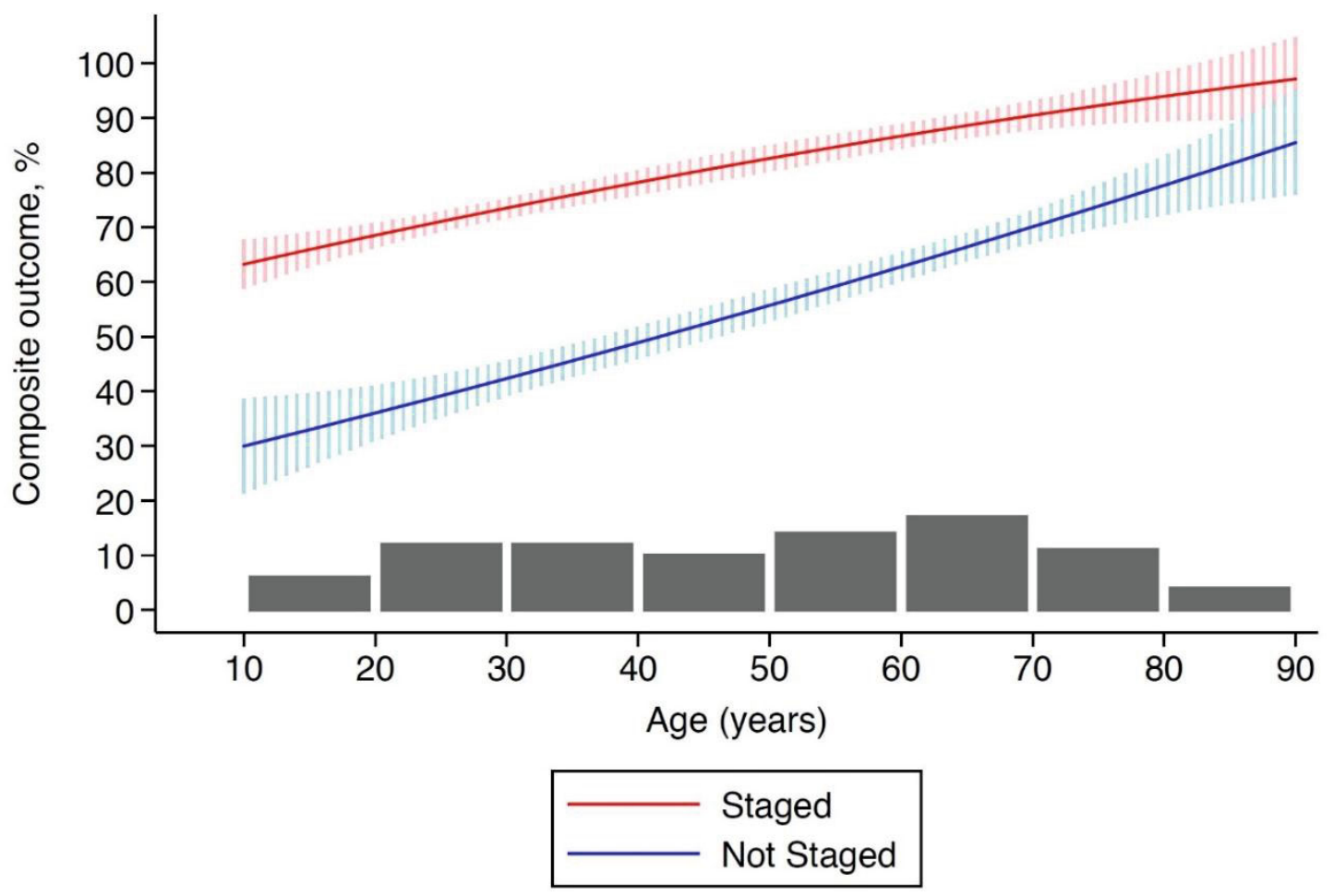




\section{Novel Front Bar Removal to Facilitate Airway Management with a Leksell Headframe in}

\section{Situ}

Tara Dalby; Christian Iorio-Morin²; Suneil Kalia²; Lashmi Venkatraghavan"; Michael Dinsmore ${ }^{1}$

1 Department of Anaesthesia and Pain Medicine, Toronto Western Hospital, University Health Network, University of Toronto, Toronto, ON, Canada

2 Department of Neurosurgery, Division of Surgery, Toronto Western Hospital, University Health Network, Toronto, ON, Canada

Background: Sterotactic headframes, such as the Leksell G model, are required for numerous neurosurgical procedures (Figure 1). In particular, this frame is used for MRI-guided Laser Interstitial Thermal Therapy (MRgLITT), a procedure requiring multiple patient transfers to allow for interoperative imaging and treatment (1). Therefore, loss of the airway during the procedure presents a real possibility and may present a major airway challenge for the Anesthetist as this Leksell frame is designed with a straight front bar which completely obscures oral access. Although previous papers have suggested the entire head frame to be removed during an airway emergency (2), we describe a novel method to remove only the front bar. Therefore, the objective of this paper is to first examine the ease of intubation with the stereotactic headframe in situ and secondly to describe and compare a novel approach to rapidly remove only the front bar.

Materials and Methods: Ethics approval was not applicable because the study did not involve human or animal research. This is an observational study where a Leksell $G$ frame with the straight front bar was secured on a mannequin. Anaesthesiologists from a single centre were asked to intubate the mannequin using a CMAC 3 with the frame fully in situ and again with only the front bar removed. In addition, Neurosurgical staff were separately timed removing the entire frame and again removing only the front bar using a newly described technique.

Results: 18 Anaesthesia personnel participated in the study as well as 4 neurosurgeons. All anaesthesia personell were able to intubate the mannequin with both the leksell frame on and with the front bar removed. The average time to intubate the mannequin in the frame was 23.5 (11.4) seconds and with the front bar removed, 10.9 (2.5) seconds; $p<0.001$. The average time taken to remove just the front bar by the neurosurgeons was 35.4 (7.3) seconds compared to an average of 83.3 (18.6) seconds; $p<0.001$ to remove the headframe entirely.

Conclusion: Although it is still possible to intubate with the Leksell frame straight front bar in situ, it is much faster to intubate with the front bar removed. As well, difficult airway situations requiring complex airway manipulation using this frame will be greatly facilitated by removing the front bar. Additionally, removing the front bar takes less than half the time than removing the entire headframe. The other benefit, is that detailed surgical planning is completed with the frame in place and therefore having to remove the entire frame would negate the entire procedure, however, removing only the front bar would allow the procedure to continue once the airway was again secured. Therefore, this new method should be considered when developing airway management in Leksell frame guidelines. 


\section{REFERENCES:}

1. Jimenez-Ruiz F, Arnold B, Tatsui C, Cata JP, Perioperative and Anaesthetic Considerations for Neurosurgical Laser Interstitial Thermal Therapy Ablations, J Neurosurg Anaesthesiol, 30:10-17, 2018

2. Brockerville M, Unger Z, Rowland NC, Sammartino F, Manninen PH, Venkatraghavan L, Airway management with a stereitactic headframe in situ- a mannequin study, J Neurosurg Anaesthesiol, 30:44-48, 2018

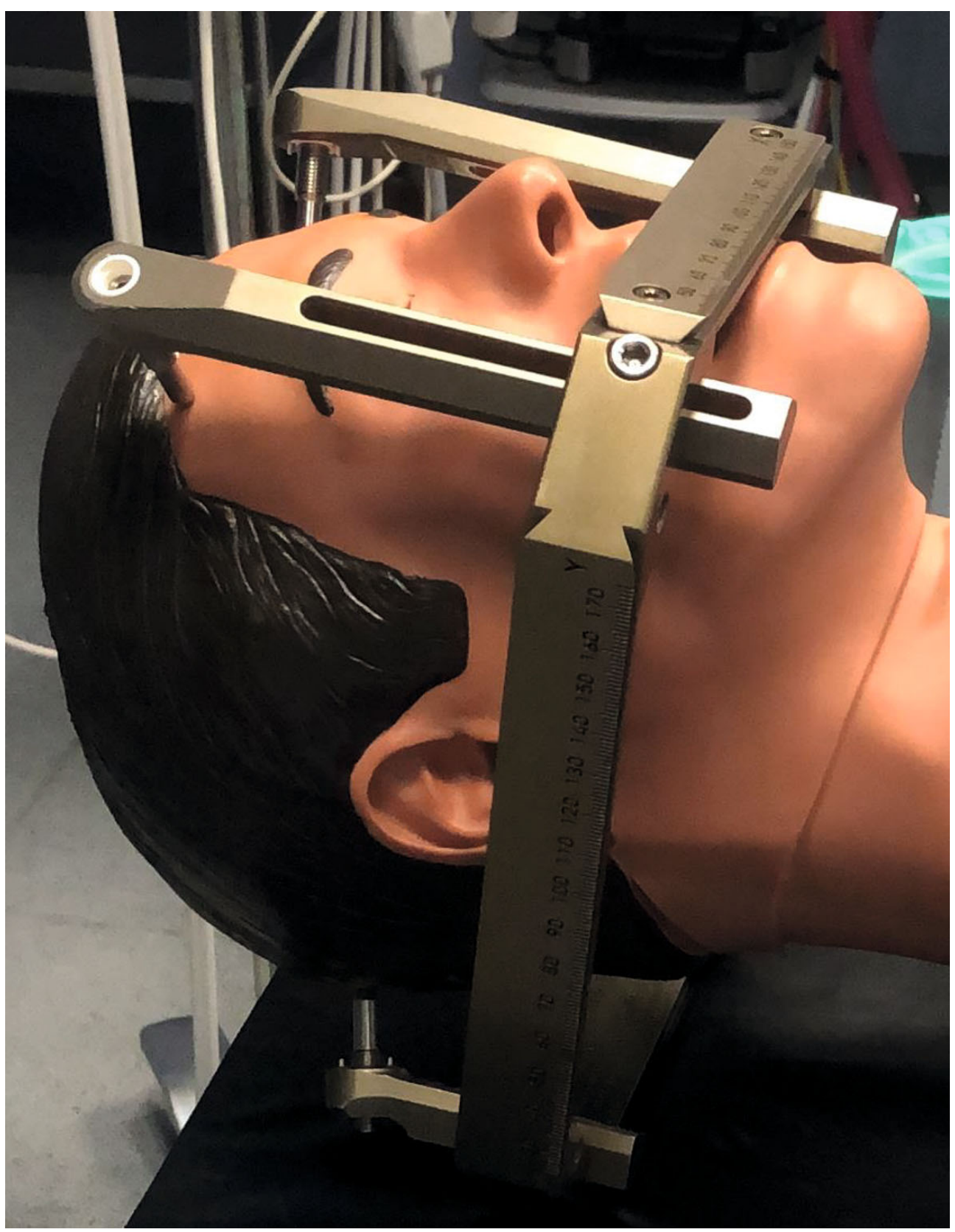




\section{Seizures During Awake Craniotomy: A Retrospective Study on Incidence and Outcomes}

Gabriel Paquin-Lanthier ${ }^{1}$; Abigael Daniels ${ }^{1}$; Kok Weng Leong ${ }^{1}$; Lashmi Venkatraghavan ${ }^{1}$; Michael Dinsmore ${ }^{1}$; Hirokazu Takami ${ }^{2}$; Demitre Serletis ${ }^{3}$; Mark Bernstein ${ }^{2}$

1 Department of Anesthesiology and Pain Medicine, Toronto Western Hospital, University of Toronto, Toronto, ON, Canada

2 Department of Surgery, Division of Neurosurgery, Toronto Western Hospital, University of Toronto, Toronto, ON, Canada

3 Section of Neurosurgery, Health Sciences Centre, University of Manitoba, Winnipeg, MB, Canada

Introduction: Intraoperative seizures are one of the worrisome complications during awake craniotomy (AC) with reported incidences between 3 and $30 \%{ }^{1}$. Intraoperative seizures may lead to loss of patient cooperation, airway complications, and unplanned conversion to general anesthesia (GA). The aim of this study was to characterize the incidence, causes, management and postoperative evolution of intraoperative seizures during ACs at our institution.

Methods: Ethics approval was obtained from the local REB. A retrospective review of all ACs performed by a single surgeon in our institution from 2004 to 2018 was done to identify cases of intraoperative seizures. Data collected included patient demographics, surgical pathology, incidence, presentation, management of intraoperative seizures and postoperative disposition of the patient. Data were analyzed and presented descriptively as either absolute number and/or $\%$ as indicated.

Results: 706 ACs were performed between 2004 and 2018, and 25 patients had intraoperative seizures, with an overall incidence of $3.5 \%$. All seizures were focal except for one generalized seizure. The baseline diagnosis was high-grade glioma in 9 patients, metastasis or radiation necrosis in 9 patients, and other pathologies in 7 patients. A preoperative diagnosis of seizures was present in 13 patients (52.0\%), all of whom received antiepileptic medication preoperatively. Seizures were associated with intraoperative electrical stimulation in 21 patients $(84.0 \%)$. Data on intraoperative seizure treatment was not available in 6 patients $(24.0 \%)$.

Seizures stopped spontaneously in 6 cases $(24.0 \%)$, needed cold saline irrigation of the brain in 8 cases (32.0\%; cold saline alone in one case, combined with propofol or benzodiazepines in 7 cases), propofol bolus in 10 cases (40.0\%), and benzodiazepines in two cases. Phenytoin was used with propofol in one case, and midazolam in one case. One patient required conversion to GA in the setting of a possible venous air embolism. No other airway related complication was recorded. Two patients had recurring seizures in PACU. 12 patients $(48.0 \%)$ presented a new neurologic deficit in post-op, while 4 of 23 patients (17.4\%) with available follow-up data still had a persisting deficit on follow-up. 8 patients were discharged from PACU to day surgery, 9 patients to the floor, 4 patients to the ICU, and 4 patients had missing discharge data. Three patients scheduled for day surgery had an unplanned admission to the hospital.

Conclusions: Our study from a large volume of awake craniotomy cases show that the incidence of intraoperative seizures is about 3.5\%. Almost all seizures were focal in nature and a majority were caused by electrical stimulation of the brain. Most seizures were controlled without converting to general anesthesia, and were not associated with long-term sequelae. Further analysis is underway comparing this series of patients with patients who did not have 
seizures to characterize risk factors of seizures in this setting.

\section{REFERENCES:}

1. Sewell, D. \& Smith, M. Awake craniotomy: Anesthetic considerations based on outcome evidence. Curr. Opin. Anaesthesiol. 32, 546-552 (2019). 
The Automated Nerve Monitor Reduces Neurological Deficits and Improve Quality of Life in Total Shoulder Arthroplasty Surgery: A Prospective Blinded, Randomized Controlled Study

Jason Chui ${ }^{1}$; Moaz BY Chohan ${ }^{1}$; John M Murkin ${ }^{1}$; Max Rachinsky; Shalini Dhir ${ }^{1}$; George Athwal $^{2}$; Ken Faber'; Darren Drosdowech ${ }^{2}$

1 Department of Anesthesia \& Perioperative Medicine, Schlich School Medicine and Dentistry, University of Western Ontario, Canada

2 Department of Orthopedic Surgery, Schlich School Medicine and Dentistry, University of Western Ontario, Canada

Background: Evoked potential (EP) monitoring is routinely used to prevent neurological injury in various surgical settings globally. However, its controversial efficacy and cost-effectiveness of EP monitoring has triggered the scrutiny of the health care funders (1-6). To address these limitations, we performed a prospective, blinded, randomized controlled trial to assess the efficacy of using an automated nerve monitor $(E P A D \circledR$, SafeOp Surgical) $(7-8)$ to reduce neurological injury in patient undergoing total shoulder arthroplasty (TSA).

Methods: Ethics approval was obtained from the local REB. Patients undergoing TSA in a single tertiary institute at Canada were randomized into either the automated nerve monitor or no monitor groups at 1:1 ratio. The patients and outcome assessors were blinded. The primary outcome was intraoperative nerve injury burden as assessed by the cumulative duration of nerve alert. The secondary outcomes were the neurological deficits and shoulder functional scores (ASES score) of the operative arm, and the quality of life health state index (EQ5D-5L) at postoperative 2 weeks, 6 weeks and 3 months. We further assessed the relationship between the postoperative outcomes and the day(s) since the study commenced using linear regression analysis.

Results: From September 2018 to July 2019, 213 patients were screened, of which 200 patients were randomized. There was no significant difference of the cumulative duration of intraoperative nerve alert between the nerve monitored and the control groups (mean (SD): 12.7 (19.4) and 15.5 (21.8) minutes; $p=0.54$ ). There were no differences of all the secondary outcomes between two groups, including postoperative neurological deficits, shoulder functional scores, and quality of life health state index at postoperative 2 weeks, 6 weeks and 3 months. However, there were statistically significant trends of reduction of the neurological deficits $(\beta=$ 0.0009 (95 Cl: -0.0015 to -0.0002$), p<0.01)$ and improvement of the quality of life $(\beta=0.0002$ (95 Cl: 0.00003 to 0.0005$), p=0.02$ ) over the study period (Fig. 1).

Conclusion: The use of automated nerve monitor was associated with reduction in postoperative neurological deficits, and improvement in quality of life in both groups over the study period. This outcome benefit was corroborated by the clinical observation that the surgeons modified their surgical technique in response to the real-time feedback provided by the automated nerve monitor, suggesting either a learning or Hawthorne effect. Importantly, these benefits were found in patients who were not being monitored (i.e. control group), as well as these surgical techniques improvement were seen even in a group of highly experienced surgeons participating in this study. This study also represents an important initiative to overcome the current logistic constraints that hamper the application of EP monitoring that may 
benefit many high-risk surgical patients worldwide.

\section{REFERENCES:}

1. Hadley MN, Shank CD, Rozzelle CJ, Walters BC. Guidelines for the Use of Electrophysiological Monitoring for Surgery of the Human Spinal Column and Spinal Cord. Neurosurgery 2017;81(5):713-32.

2. Vogel R, Balzer J, Gertsch J, et al. Letter: Guidelines for the Use of Electrophysiological Monitoring for Surgery of the Human Spinal Column and Spinal Cord. Neurosurgery 2018;82(6):E190-1.

3. Hadley MN, Shank CD, Rozzelle CJ, Walters BC. In Reply: Guidelines for the Use of Electrophysiological Monitoring for Surgery of the Human Spinal Column and Spinal Cord. Neurosurgery 2018;82(6):E192-3.

4. SALA F, Skinner SA, Arle JE, et al. Letter: Guidelines for the use of Electrophysiological Monitoring for Surgery of the Human Spinal Column and Spinal Cord. Neurosurgery 2018;83(2):E82-4.

5. Ney JP, van der Goes DN. Letter: Guidelines for the Use of Electrophysiological Monitoring for Surgery of the Human Spinal Column and Spinal Cord. Neurosurgery 2018;83(2):E78-9.

6. Wilkinson M, Houlden D. Letter: Guidelines for the Use of Electrophysiological Monitoring for Surgery of the Human Spinal Column and Spinal Cord. Neurosurgery 2018;83(2):E74-5. 7. Chui J, Murkin JM, Drosdowech D. A Pilot Study of a Novel Automated Somatosensory Evoked Potential (SSEP) Monitoring Device for Detection and Prevention of Intraoperative Peripheral Nerve Injury in Total Shoulder Arthroplasty Surgery. J Neurosurg Anesthesiol 2019;31(3):291-8.

8. Chui J, Murkin JM, Turkstra T, McKenzie N, Guo L, Quantz M. A novel automated somatosensory evoked potential (SSEP) monitoring device for detection of intraoperative peripheral nerve injury in cardiac surgery: a clinical feasibility study. J Cardiothorac Vasc Anesth 2017;31:1174-82. 

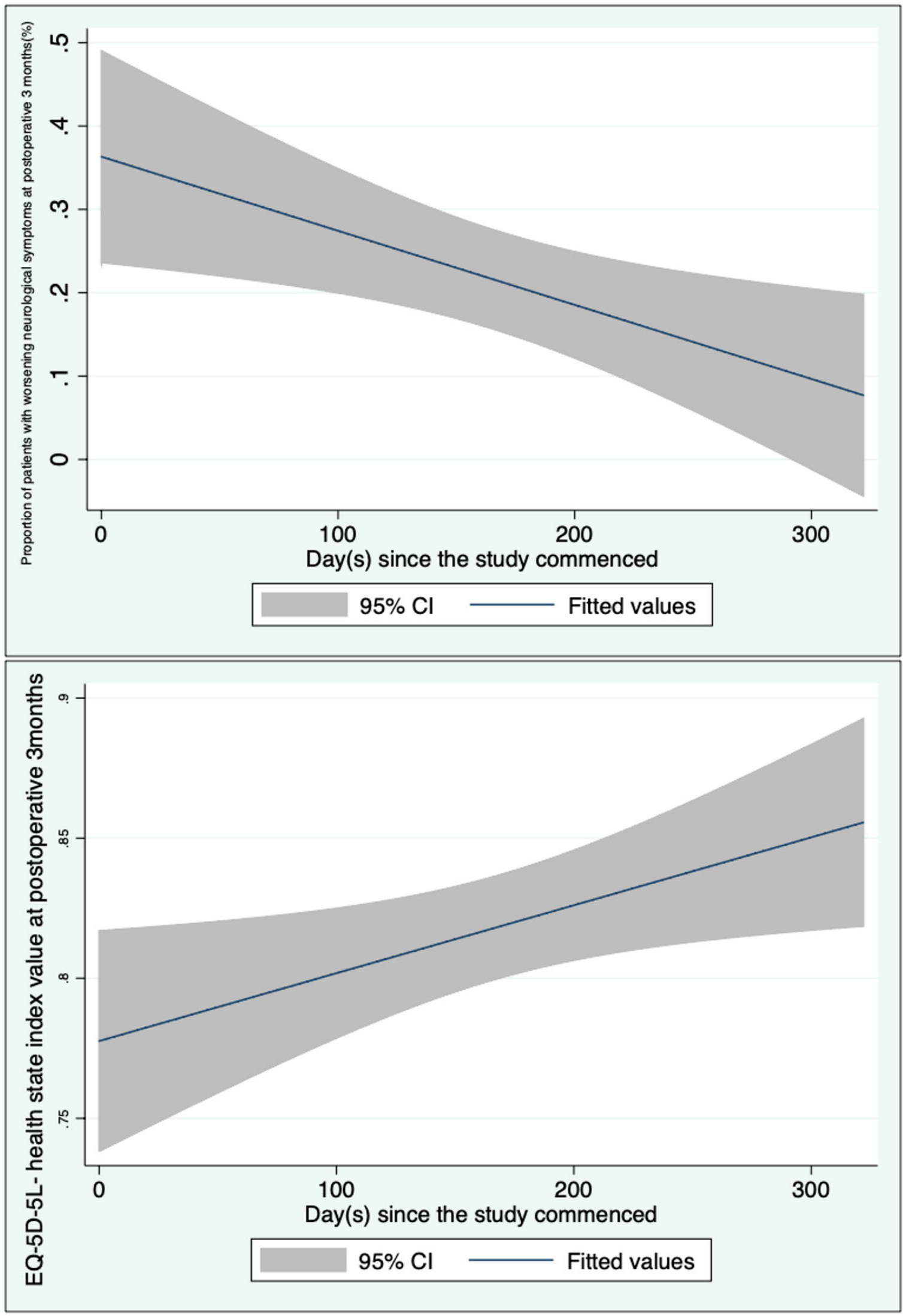


\section{OBSTETRIC ANESTHESIA}

\section{A Retrospective Cohort Study of the Association Between Labour Epidural Analgesia and Postpartum Depression in Primiparous Women}

Sherry Jin ${ }^{1}$; Christy G. Woolcott ${ }^{2}$; Allana Munro ${ }^{1}$; Chantal Houser ${ }^{1}$; Ronald B. George ${ }^{3}$

1 Department of Anesthesiology, Pain Management, and Perioperative Medicine, Dalhousie University, Halifax, Canada

2 Department of Pediatrics, Department of Obstetrics and Gynecology, Dalhousie University, Halifax, Canada

3 Department of Anesthesia and Perioperative Care, University of California San Francisco, San Francisco, USA

Introduction: The prevalence of postpartum depression (PPD) is $6.5-12.9 \%$ and it is a significant public health concern that affects the mother, her child, and the family unit. ${ }^{1}$ PPD has been associated with the intensity of labour pain ${ }^{2}$ and severity of acute postpartum pain ${ }^{3}$, making pain a potentially modifiable risk factor for PPD. Thus far in the literature, however, the relationship between labour analgesia and PPD is unclear.4 While some studies have reported an association between labour epidurals and reduced rates of $\mathrm{PPD}^{5-7}$, others have shown no association between the two ${ }^{8,9}$. The objective of this study was to examine the association between labour epidural analgesia (LEA) and PPD among primiparous women undergoing vaginal delivery.

Methods: Ethics approval was obtained from the local REB. A retrospective cohort study was completed using a provincial perinatal database. The database was searched for all primiparous women who delivered a liveborn singleton infant between 2003-2018. Those women who experienced PPD following their first delivery were identified by searching for a history of PPD in the perinatal records of their second pregnancy. Relevant demographic, medical, obstetric, and anesthetic information was collected from the database. Odds ratios $(\mathrm{OR})$ for the association between LEA and PPD were estimated with logistic regression to control for potential confounding variables.

Results: A total of 35,437 primiparous women were identified who had information about a history of PPD in the record of their second pregnancy. Of this total, $67.3 \%(n=23,862)$ received LEA and $3.7 \%(n=1,296)$ developed PPD. Women who received LEA had a slightly increased odds of developing PPD compared to women who did not receive LEA (OR 1.38, $95 \% \mathrm{Cl} 1.22$ to 1.57). Further adjustment for other potential confounders (e.g. body mass index, pre-existing anxiety) did not affect the estimated OR.

Discussion: This retrospective cohort study showed an association between LEA and PPD among primiparous women. The findings call into question the hypothesis that LEA decreases the risk for PPD and further illustrates the complexities of PPD. 


\section{REFERENCES:}

1. N Engl J Med. 2016;375:2177-86.

2. L'Encephale. 2007;33(5):805-10.

3. Pain. 2008;140(1):87-94.

4. Can J Anesth. 2020; [in press].

5. Anesth Analg. 2014;119(2):383-92.

6. Anesth Analg. 2018;126(5):1598-605.

7. Eur J Anaesthesiol. 2019;36(10):745-54.

8. Local Reg Anesth. 2017;10:99-104.

9. Int J Obstet Anesth. 2018;35:10-6

Table 1: The association between epidural use and PPD

\begin{tabular}{c|c|c|c|c} 
& $\mathrm{N}$ & $\mathrm{N}$ cases (\%) & Unadjusted OR (95\% CI) & Adjusted* OR (95\% CI) \\
\hline No LEA & 11576 & $346(2.9)$ & 1.00 (ref) & 1.00 (ref) \\
\hline LEA & 23862 & $956(4.0)$ & $1.38(1.22$ to 1.56$)$ & $1.38(1.22$ to 1.57$)$ \\
\hline
\end{tabular}

*Adjusted for year of birth, maternal age 
Acupuncture for Pain Control After Caesarean Section - Randomized Blinded PlaceboControlled Investigation with Non-Randomized Arm

Taras I. Usichenko ${ }^{1,2}$; Berthold Henkel ${ }^{1}$; Catharina Klausenitz ${ }^{3}$; Marek Zygmunt ${ }^{4}$; Thomas Hesse $^{1}$; Guillermo Pierdant ${ }^{4}$; Mike Cummings ${ }^{5}$; Klaus Hahnenkamp ${ }^{1}$

1 Dept. of Anesthesiology, University Medicine of Greifswald, Germany

2 Dept. of Anesthesia, McMaster University, Hamilton, Canada

3 Dept. of Radiology, University Medicine of Greifswald, Germany

4 Dept. of Gynecology and Obstetrics, University Medicine of Greifswald, Germany

5 British Medical Acupuncture Society, London, United Kingdom

Background: The pharmacological approach alone for pain control after caesarean section (CS) is often insufficient $(1,2)$. Acupuncture is a promising method for mitigating postoperative pain and reducing postoperative opioid requirements $(3,4)$. The aim was to investigate the effectiveness of acupuncture as an adjunctive therapy for pain control after CS compared with placebo acupuncture and standard therapy alone.

Methods: Ethics approval was obtained from the local REB. Patients scheduled to elective CS under spinal anesthesia, were enrolled according to defined inclusion criteria and randomized into the acupuncture group $(A G, N=60)$ or placebo acupuncture group ( $P G, N=60)$. Each patient in AG received auricular and body acupuncture with 20 indwelling fixed needles according to a previously validated protocol of acupuncture for postoperative analgesia $(3,5)$. Patients in the PG were treated with placebo needles in addition to standard pain treatment. Another 60 consecutive patients, who were included according to eligibility criteria and received the standard postoperative analgesia alone, constituted a non-randomized "no intervention" ( $\mathrm{NI}$ ) control group. The following outcomes were recorded: pain intensity on an 11point verbal rating scale (VRS-11, where $0=$ no pain and $10=$ maximal pain); analgesics consumption; rate of analgesia-related side effects; time to mobilization and Foley catheter (FC) removal after CS; patients' compliance and satisfaction with treatment of pain on a 5-point VRS (VRS-5, where 1=excellent; 5=bad).

Results: All 180 enrolled patients were included in the "intention-to-treat" analysis. Pain intensity on movement in $A G$ at the first postoperative day was $4.7 \pm 1.7$ vs. 6.0. \pm 2.0 in $P G$ (mean \pm standard deviation; VRS-11; $P=0.001$ ). The patients from AG demonstrated faster mobilization and removal of $F C$ than those in $P G(P<0.01$; Figure 1). Patients from $\mathrm{NI}$ reported the same pain intensity on movement as patients from $P G(5.8 \pm 1.7 ; P=0.8)$ and delayed mobilization and removal of FC in comparison with AG and later ambulation than PG $(P<0.05)$. Analgesic consumption and other measurement parameters were comparable among the study groups.

Conclusion: Acupuncture relieves clinically relevant pain and accelerates the mobilization of patients after caesarean section. With additional consideration of personnel and time expenditure, acupuncture can be recommended for the routine use for postoperative pain therapy in patients after an elective caesarian section. 


\section{REFERENCES:}

3. Lavoie \& Toledo. Multimodal postcesarean delivery analgesia. Clin Perinatol 2013;40:44355.

4. Marcus et al. Quality of pain treatment after caesarean section: results of a multicentre cohort study. Eur J Pain 2015;19:929-39.

5. Sun et al. Acupuncture and related techniques for postoperative pain: a systematic review of randomized controlled trials. Br J Anaesth 2008;101:151-60.

6. Tedesco et al. Drug-Free Interventions to Reduce Pain or Opioid Consumption After Total Knee Arthroplasty: A Systematic Review and Meta-analysis. JAMA Surg 2017;152:e172872.

7. Hesse et al. Acupuncture for pain control after Caesarean section: a prospective observational pilot study. Acupunct Med 2016;34:14-9.

\section{Figure 1}

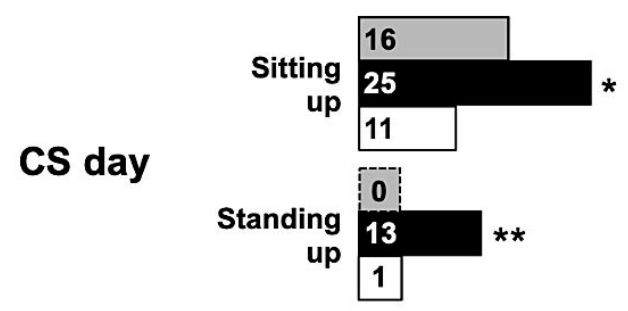

\section{First post-OP day}
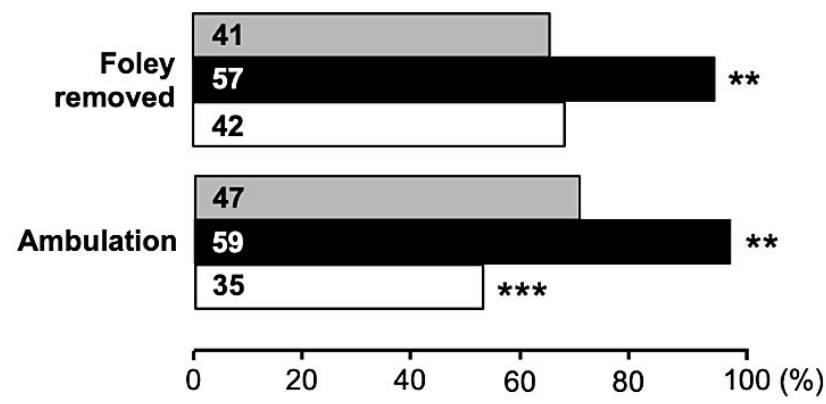

Results of patients mobilization after Caesarean Section (CS) on the day of CS and on the first postoperative day. Data given as per cent from total number of patients; numeric values are absolute number of patients; *means $P=0.03$ for AG vs NI; ** $P<0.01$ for AG vs PG and AG vs NI; *** $P=0.03$ for PG vs NI; Fisher's exact test, $P$-values Bonferroni-adjusted. 


\section{Cesarean Section in a Parturient with Neurofibromatosis 1 and Harrington Rods: Is GA} the Only Option?

Sherry $\operatorname{Jin}^{1}$; Vishal Uppal ${ }^{1}$; Allana Munro ${ }^{1}$; Matthew Simms ${ }^{1}$

1 Department of Anesthesiology, Pain Management, and Perioperative Medicine, Dalhousie University, Halifax, Canada

Introduction: Neurofibromatosis 1 (NF1) is a neurocutaneous disorder, caused by a mutation in a tumour suppressor gene, with an incidence of 1 in 2500-3300. ${ }^{1}$ Most patients present with café au lait spots and cutaneous neurofibromas. Neurofibromas also develop along peripheral nerves and, less commonly, in the central nervous system (CNS). ${ }^{1}$ Neuraxial anesthesia (NA) can be complicated by the presence of peripheral neuropathy, spinal root compression, and scoliosis. ${ }^{1,2}$ Additionally, these patients may have difficult airways due to the presence of intraoral tumours and cervical spine involvement. ${ }^{1}$ The sequelae of NF1 present several challenges for the delivery of obstetrical anesthesia including the decision to pursue NA or general anesthesia (GA).

Case Presentation: A 31-year-old G2P1 with NF1 underwent an elective repeat caesarean delivery (CD) for breech presentation. She previously had an urgent $C D$ due to non-reassuring fetal heart rate under GA. She had no known history of intracranial or spinal tumours and no neurologic deficits. She had Harrington rods placed from T4-L3 for correction of scoliosis. Plain radiographs confirmed the presence of spinal instrumentation and residual thoracolumbar scoliosis. On physical exam, her airway was reassuring and she had limited flexion in her thoracic and lumbar spine. Neuraxial ultrasound revealed patent lumbar spaces with no subcutaneous tumours appreciated. While the patient expressed interest in NA, informed consent for GA was obtained due to the potential for CNS involvement, the absence of MRI neuroimaging, and the high risk for inadequate block. A healthy baby girl was delivered without perioperative complications.

Conclusion: There are few case reports on the anesthetic management of parturients with NF1. In most cases, an MRI of the spine was obtained to assess for spinal tumours prior to neuraxial anesthesia ${ }^{3,4}$ or a GA was performed in the absence of neuroimaging ${ }^{5}$. There is no literature on the management of parturients with both NF1 and Harrington rods. Modern Harrington rods are MRI compatible but their presence distorts the images. ${ }^{6}$ In our case, GA was selected because of the paucity of information surrounding the safety of NA without preoperative MRI and the high risk for failed neuraxial blockade. However, with appropriate consent, NA may have been reasonable. Only one complication from a neuraxial procedure in a parturient with NF1 has been reported. ${ }^{7}$ The patient developed an epidural hematoma despite no evidence of neurofibroma on MRI. ${ }^{7}$ Ultrasound may be an alternative tool to identify large masses in the trajectory of needle placement for NA, but further research into the accuracy of neuraxial ultrasound in detecting neurofibromas is warranted. 


\section{REFERENCES:}

1. BJA. 2001;86(4):555-64.

2. Brain. 1999;122(Pt 3):473.

3. Can J Anaesth. 1995;42(5):420-4.

4. Proc (Bayl Univ Med Cent). 2018;31(1):92-3.

5. J Clin Anesth. 2007;19:405-7.

6. Eur J Radiol. 2015;84:450-7.

7. BJA. 2001;87(6):932-4.

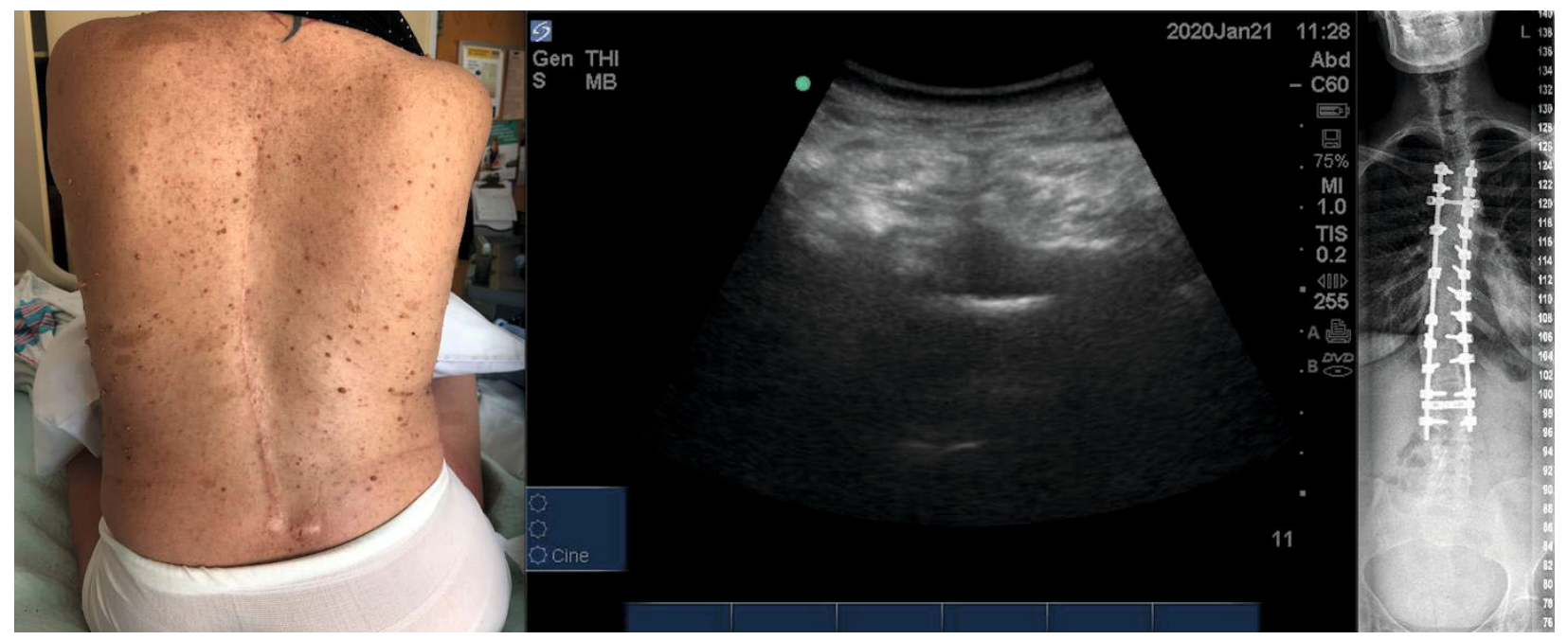




\title{
Electrocardiographic Changes Associated with Epidural Infusion of Ropivacaine in a
} Patient with SCN5A Sodium Channel Mutation

\author{
Ana Sjaus ${ }^{1}$; Lynne McLeod ${ }^{2}$
}

1 Department of Women's and Obstetric Anesthesia, IWK Health Centre, Halifax, NS Canada

2 Department of Obstetrics and Gynecology, IWK Health Centre, Halifax, NS Canada

Introduction: Inherited ion channelopathies can be associated with a variety of abnormalities of cardiac rhythm and structure. The SCN5A gene encodes a subunit of the cardiac voltage gated sodium channel. Long QT, Brugada syndrome, dilated cardiomyopathy, arrhythmogenic right ventricle have been reported in patients with this gene mutation [1].

Benefits of epidural analgesia include decreasing the risk of cardiac dysrhythmia due to attenuation of sympathetic tone and facilitating obstetric intervention. While less likely than bupivacaine to be cardiotoxic, ropivacaine is also a $\mathrm{Na}+$ channel blocker that can potentially exacerbate ion channel dysfunction. Previously reported use of ropivacaine for neuraxial labor analgesia in a patient with SCN5A mutation was limited to 6.5 hours of labor [2]. This case report describes safe labor analgesia with epidural ropivacaine for 21 hours despite the development of non-specific ECG changes in a patient with newly diagnosed SCN5A mutation.

Case Presentation: Patient consent was obtained for publication of this case. An otherwise healthy primipara reported episodes of dyspnea and palpitations in pregnancy. Due to family history of Long QT Syndrome and dilated cardiomyopathy, she was investigated for inherited conduction abnormality.

Electrocardiogram and Holter monitor showed sinus tachycardia, RSr' pattern with normal QRS duration and runs of ventricular tachycardia. QT duration was normal. Echocardiogram revealed wall motion abnormality of the right ventricle but normal systolic function. Exact diagnosis was not known at the time of anesthetic consultation at 36 weeks of gestation. Early epidural was recommended due to poor tolerance of tachycardia and the risk of stress related QT prolongation. Avoidance of QT prolonging medication was recommended.

The presence of SCN5A mutation was confirmed several days prior to admission in labor. Labor epidural was induced and maintained using dilute solution of ropivacaine and fentanyl according to standard protocol. As the patient's particular phenotype was unconfirmed, conversion to IV opioid was planned in the event of long QT, bradycardia or ST elevation characteristic of Brugada syndrome. Continuous monitoring revealed persistent sinus tachycardia with evolving partial right bundle branch block and ST inversion. QT interval remained normal. The patient reported several episodes of dyspnea consistent with prior complaints. The duration of epidural infusion was 21 hours. Monitoring was continued for 8 hours post-partum until ECG changes resolved to baseline. The neonate was found to have ST changes consistent with Brugada syndrome.

Discussion: SCN5A mutation of the sodium channel can lead to dysrhythmias including cardiac arrest. Different SCN5A phenotypes can overlap in the same patient. Confirming the specific phenotype and clinical features can help predict consequences of neuraxial analgesia, provoking medications and clinical conditions. A multidisciplinary approach that includes careful monitoring is necessary for safe maternal and neonatal management. 


\section{REFERENCES:}

1. Journal of Arrhythmia 2013;Apr 29(2): 71-76

2. Case Reports in Anesthesiology 2016, Article ID 9278409

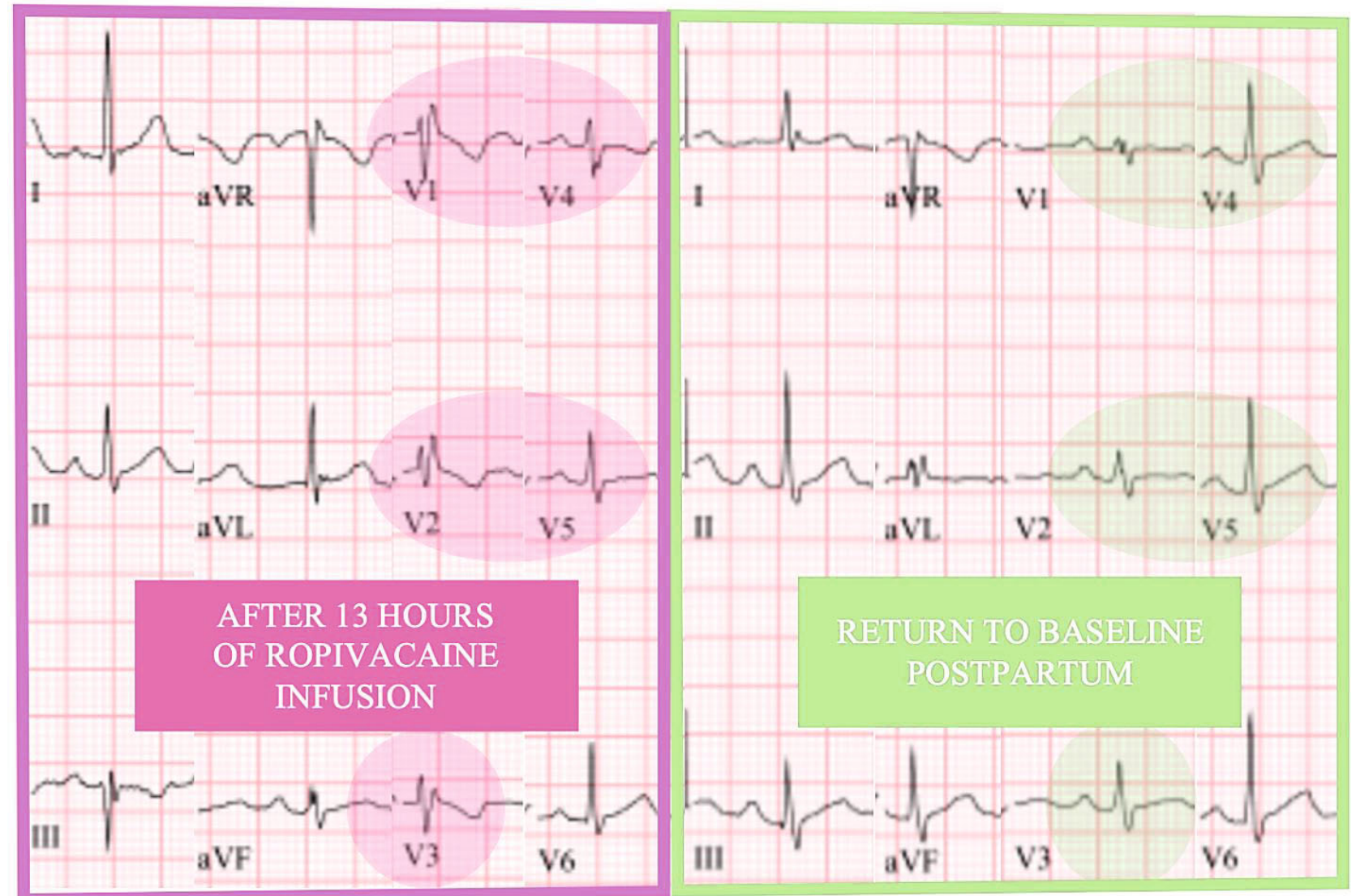


Engagement with a Perioperative Mobile Application for Mothers undergoing Cesarean Delivery: A Prospective Cohort Study

Janny Xue Chen Ke $\mathrm{K}^{1}$; Ronald B. George ${ }^{1,2}$; Lori Wozney ${ }^{3}$; Allana Munro ${ }^{1,3}$

1 Department of Anesthesiology, Pain Management, and Perioperative Medicine, Dalhousie University, Halifax, Canada

2 Department of Anesthesia and Perioperative Care, UCSF, San Francisco, USA 3 IWK Health Centre, Halifax, Canada

Introduction: Giving birth is the most common reason for hospital admission, with Cesarean Delivery (CD) being the most frequently performed inpatient surgery (1-3). Lack of knowledge about birth complications and potential warning signs contribute to preventable maternal mortality (4). Through a needs assessment and iterative design process involving patients and obstetric anesthesiologists, we previously developed C-Care, a mobile application for CD (5) focused on perioperative education and self-monitoring of anesthetic complications. This study aimed to measure the extent of patient engagement with the mobile application during a pilot implementation, and obtain quantitative and qualitative feedback regarding feasibility.

Method: Ethics approval was obtained from the local REB. With written patient consent, we conducted a prospective cohort study of patients $\geq 18$ years $(N=36)$ having an elective $C D$. The usage data from $\mathrm{C}$-Care was tracked and recorded for 30 days after CD. On postoperative days $1-5$, patients received a short self-monitoring questionnaire. Fourteen days after surgery, patients received an online survey regarding overall satisfaction, potential impact on care, usability and feasibility of C-Care. Qualitative and quantitative metrics were used to evaluate patient engagement. Primary outcomes included: number of views of education topics, completion of self-checks, total visits, satisfaction score, and recommendation to others. Secondary outcomes included: rank of education topics by frequency of views, timing of selfmonitoring and mobile application visits, incidence of positive self-check symptoms, knowledge delivery, and feedback for improvement.

Results: Thirty-six patients completed the study from 2018 to 2019 . Each participant viewed $4.5 \pm 2.5$ education topics and visited the application $19.4 \pm 14.1$ times within 30 days postoperatively. The median number of self-monitoring questionnaires completed was $3 \pm 1.3$ (out of 5). The top three most commonly viewed patient education topics were "Controlling Pain" on multimodal analgesia, an overview of the postoperative course named "The First Few Days", and "Contact Information". Visits to the application were highest within the first week, with the most being on the first two days. Of the 18 respondents who completed the day 14 survey, $83 \%$ $(\mathrm{N}=15)$ patients recommended $\mathrm{C}$-Care to other women, and the median patient satisfaction score was 7.5 (range 2-10). Patients responded that C-Care provided them knowledge about CD and anesthesia ( $N=17,94 \%)$, potential complications to monitor for $(N=15,83 \%)$, and the recovery process after $\mathrm{CD}(\mathrm{N}=15,83 \%)$. Themes related to improvement included the need for more content, and access to the application earlier during pregnancy.

Discussion: The trends and usage data from this study increased our understanding of patient behavior with a perioperative mobile application in the setting of CD. The findings could help design more effective and tailored patient education and self-monitoring programs. 


\section{REFERENCES:}

1. Canadian Institute for Health Information. Hospital stays in Canada | CIHI [Internet]. 2019 [cited 2019 Aug 10]. Available from: https://www.cihi.ca/en/hospital-stays-in-canada 2. Healthcare Cost and Utilization Project. Most Common Hospital Inpatient Diagnoses HCUP Fast Stats [Internet]. [cited 2019 Aug 10]. Available from: https://www.hcupus.ahrq.gov/faststats/NationalDiagnosesServlet

3. Healthcare Cost and Utilization Project. Most Common Hospital Inpatient Operations HCUP Fast Stats [Internet]. [cited 2019 Aug 10]. Available from: https://www.hcupus.ahrq.gov/faststats/NationalProceduresServlet

4. Pregnancy Mortality Surveillance System | Maternal and Infant Health | CDC [Internet]. 2019 [cited 2019 May 23]. Available from:

https://www.cdc.gov/reproductivehealth/maternalinfanthealth/pregnancy-mortality-surveillancesystem.htm

5. Ke JXC, George RB, Wozney L, Chorney JL. Patient-centred perioperative mobile application in Cesarean delivery: needs assessment and development. Can J Anaesth J Can Anesth. 2019 Oct;66(10):1194-201. 


\title{
C-Care App Study Overview
}

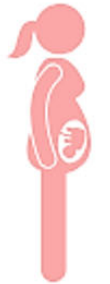

\author{
36 women undergoing \\ Cesarean delivery \\ - Consent \\ - Demographics Survey
}

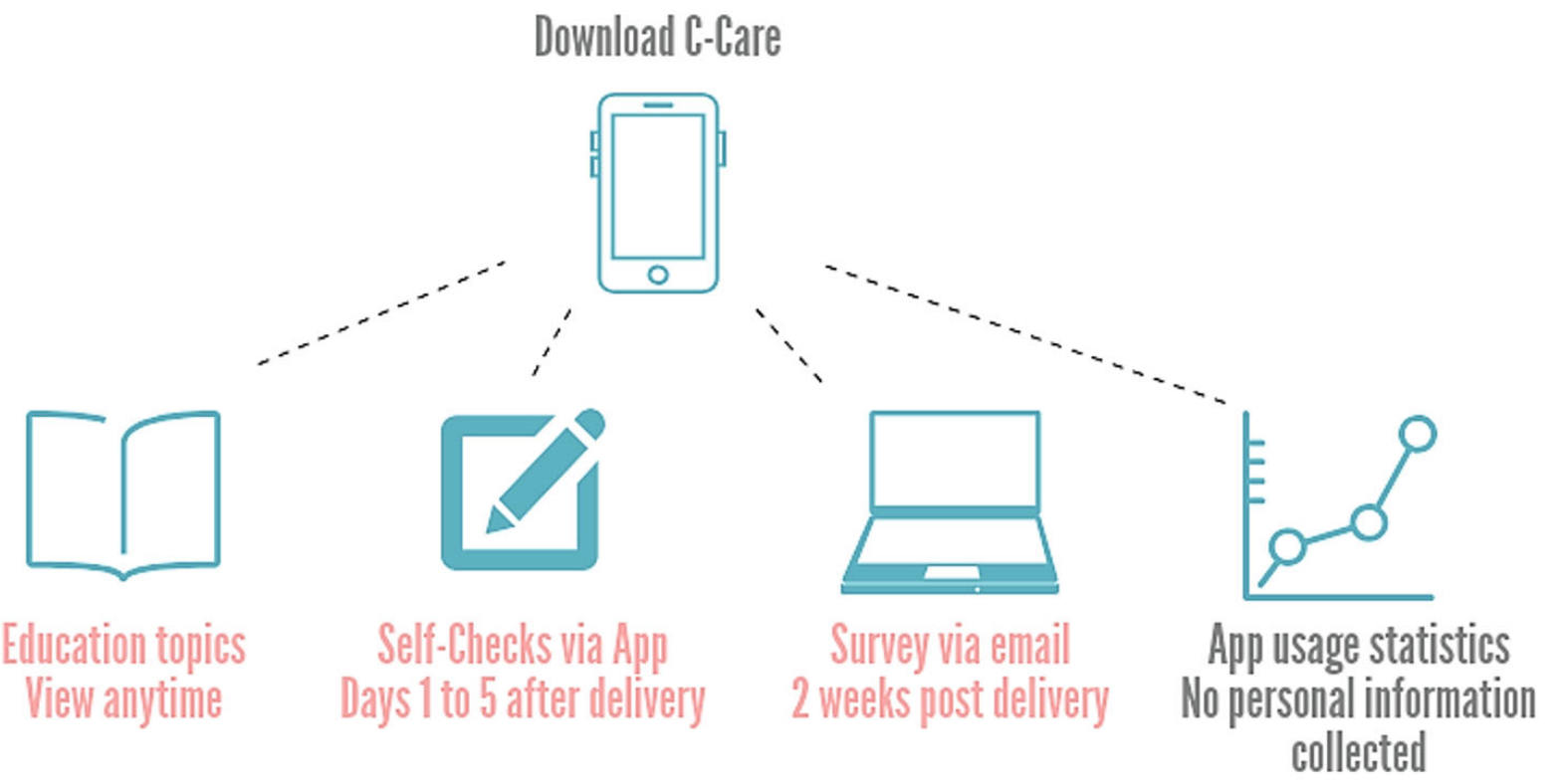

REB 1023201 Version July 2018 
Incidence and Outcomes Across Obstetrical Patients Who Receive Extracorporeal Membrane Oxygenation: A Nationwide Inpatient Study in the United States

James Wicker ${ }^{1}$; Rabail Chaudhry ${ }^{2}$; Talha Mubashir ${ }^{3}$; Laveena Munshi ${ }^{4}$ Mrinalini Balki ${ }^{1}$

1 Department of Anesthesia and Pain Management, Mount Sinai Hospital, University of Toronto, Toronto, Canada

2 University of Toronto, Department of Anesthesia, Toronto, Canada

3 University of Texas, Houston, USA

4 Interdepartmental Division of Critical Care Medicine, Toronto, Canada

Introduction: The use of extracorporeal membrane oxygenation (ECMO) has increased dramatically following reports of successful use across patients with acute hypoxemic respiratory failure and circulatory shock in recent years. ${ }^{1,2}$ However, the literature reporting the experience with ECMO across the obstetrical population is sparse. Concerns unique to this population may include maternal and fetal complications. ${ }^{3}$ The objective of this study was to determine the incidence, characteristics and outcomes of pregnant patients who receive ECMO. The authors aimed to evaluate predictors associated with the need for ECMO and factors associated with death across pregnant patients who undergo ECMO.

Methods: Ethics approved not applicable because the study did not involve human/animal research. We conducted a retrospective population-based cohort study using the Nationwide Inpatient Sample with weighted estimates of US hospitalizations of obstetrical patients requiring ECMO from 2010 to 2016. Patients treated with ECMO were identified using the diagnostic and procedural codes from the International Classification of Diseases 9th and 10th Edition. We evaluated demographic and obstetrical characteristics, etiologies necessitating ECMO and outcomes. Multivariate regression models were used to identify variables associated with inhospital mortality.

Results: An estimated 5,346,517 pregnancy-based hospital discharges were identified, of which 59 discharges had an ICD-code associated with ECMO. The use of ECMO in pregnancy has increased over time, with the highest prevalence noted in 2015 (2.3 per 100,000 hospitalizations). The mean (Standard Error, SE) age of ECMO patients was 28.7 (1.3) years, with the majority being white $(51 \%)$. Two thirds of patients had a Charlson Comorbidity Index of $0(51 \%)$ or $1(25 \%)$. Across ECMO recipients, $25 \%$ had pre-existing chronic kidney disease and $17 \%$ had pre-eclampsia. Fifty-four percent of patients receiving ECMO had concomitant acute kidney injury and $46 \%$ had sepsis (Table 1). The overall in-hospital mortality was $30.5 \%$ and the mean (SD) hospital length of stay was 23.8 (3.8) days. Mean (SE) total hospital charges were \$493,972 (77,013). In our exploratory, multivariable logistic regression analysis, there were no identified demographic characteristics, patient characteristics or complications that were associated with ECMO mortality with the exception of fetal death (Table 1).

Conclusion: Approximately 1.2 per 100,000 hospitalizations during pregnancy in a US-based nationally representative data was treated with ECMO. The mortality among these patients who receive $\mathrm{ECMO}$ was $30.5 \%$ which, on average, is lower than the general medical-surgical intensive care population treated with ECMO for ARDS or cardiogenic shock. This is likely attributable to factors including age, health status and indications for ECMO requirements. 
Based on our findings, the use of ECMO is uncommon in pregnancy and is mostly associated with acute cardiorespiratory complications (including ARDS and pneumonia). Future research should focus on evaluating factors associated with ECMO complications unique to the obstetrical population as well as ECMO outcomes.

\section{REFERENCES:}

1. Pineton de Chambrun, $M$ et al. Venoarterial extracorporeal membrane oxygenation in cardiogenic shock indications, mode of operation, and current evidence. Current Opinion in Critical Care. 2019; 397-402

2. Munshi, $L$ et al. Venovenous extracorporeal membrane oxygenation for acute respiratory distress syndrome: a systematic review and meta-analysis. Lancet Respir Med. 2019; 163172

3. Agerstrand, C. et al. Extracorporeal Membrane Oxygenation for Cardiopulmonary Failure During Pregnancy and Postpartum. Ann Thorac Surg. 2016; 774-779

Table 1. Association between potential etiologies/complications with mortality in pregnant patients treated with ECMO.

\begin{tabular}{|c|c|c|}
\hline & ECMO Prevalence N (\%) & OR $(95 \% \mathrm{CI})$ \\
\hline \multicolumn{3}{|c|}{ Etiologies associated with ECMO utilization } \\
\hline Acute respiratory distress syndrome & $44(74.6)$ & $0.84(0.24-2.94)$ \\
\hline Acute kidney injury & $32(54.2)$ & $1.50(0.48-4.62)$ \\
\hline Sepsis & $27(45.8)$ & $0.93(0.30-2.82)$ \\
\hline Pneumonia & $24(40.7)$ & $0.30(0.08-1.07)$ \\
\hline Cardiogenic shock & $23(39.0)$ & $1.39(0.45-4.28)$ \\
\hline Cardiac arrest & $19(32.2)$ & $3.1(0.97-9.96)$ \\
\hline Cardiomyopathy & $17(28.8)$ & $1.97(0.60-6.46)$ \\
\hline Septic shock & $15(25.4)$ & $0.78(0.21-2.89)$ \\
\hline Obstetric shock & $15(25.4)$ & $1.19(0.34-4.18)$ \\
\hline Postpartum hemorrhage & $9(15.3)$ & $1.17(0.26-5.29)$ \\
\hline Pulmonary embolism & $3(5.1)$ & $1.15(0.10-13.5)$ \\
\hline Pulmonary hypertension & $3(5.1)$ & $5.00(0.42-59.1)$ \\
\hline Status asthmaticus & $2(3.39)$ & \\
\hline Amniotic embolism & $1(1.7)$ & \\
\hline Pulmonary hemorrhage & $1(1.7)$ & \\
\hline \multicolumn{3}{|c|}{ Potential Complications Associated with ECMO Utilization } \\
\hline RBC transfusion & $20(33.9)$ & $1.93(0.61-6.09)$ \\
\hline Fetal death & $5(8.5)$ & $11.4(1.18-111.1)$ \\
\hline Intracerebral hemorrhage & $2(3.4)$ & $2.35(0.14-39.8)$ \\
\hline
\end{tabular}

Bold font denotes a statistically significant result.

Abbreviations: $\mathrm{ECMO}=$ extracorporeal membrane oxygenation, $\mathrm{N}=$ number of patients, $\mathrm{OR}=\mathrm{Odds}$ Ratio, $\mathrm{CI}=$ confidence interval. 


\section{Platelet Membrane Dynamics: A Novel Technique to Identify Procoagulation in} Preeclampsia

Lorraine Chow ${ }^{1}$; Joshua Nicholas ${ }^{1}$; Adrienne Lee ${ }^{2}$; Alex Gregory ${ }^{1,3}$; Gary Dobson ${ }^{1}$; Alistair W Poole $^{4}$; Ejaife Agbani, ${ }^{3,5}$

1 Department of Anesthesiology, Perioperative and Pain Medicine, Cumming School of Medicine, University of Calgary, Calgary, Canada

2 Department of Medicine, Cumming School of Medicine, University of Calgary, Calgary, Canada

3 Libin Cardiovascular Institute of Alberta, Calgary, Canada

4 School of Physiology, Pharmacology and Neuroscience, University of Bristol, Bristol, United Kingdom

5 Department of Physiology and Pharmacology, Cumming School of Medicine, University of Calgary, Calgary, Canada

Introduction: Preeclampsia is characterized by hypertension and proteinuria, and is often accompanied by thrombocytopenia and subsequent coagulopathy ${ }^{1}$. The exact mechanism of platelet dysfunction is unknown, but we postulate that platelet activation may be part of the pathogenesis, rather than a sequelae of preeclampsia. Systematic analysis of procoagulant membrane dynamics (PMD) in platelets was compared.

Methods: Ethics approval was obtained from the local REB. A pilot study was performed on 26 patients: Non-pregnant control $(\mathrm{C}=9)$, healthy pregnancy $(\mathrm{HP}=9)$, and preeclampsia $(\mathrm{PE}=8)$. Pregnant participants between 24-42 weeks gestational age were recruited. Those with underlying coagulation abnormalities, or medications affecting coagulation (except for low-dose aspirin) were excluded. Platelet ATP secretion and whole blood aggregation were analyzed by standard impedance aggregometer. 4-D live-platelet confocal imaging was used to quantify markers of granular secretion and thrombin generation indicative of platelet activation on the its outer membrane ${ }^{2,3}$. Image data quantification, 3D/4D reconstruction and movie rendering were performed using Volocity ${ }^{\mathrm{TM}}$ software. Clinical outcomes of thrombosis and hemorrhage was followed for 48 hours after delivery in pregnancy samples.

Results: Hypercoagulability in pregnancy was accentuated in preeclampsia. Mean GA was $39 \pm 1$ weeks for HP and $31 \pm 4$ for PE. Mean platelet count (109/L) was $253 \pm 33,207 \pm 41$ and $186 \pm 63$ for C, HP and PE respectively. Preeclampsia platelets were significantly activated at baseline and showed major remodeling of open canalicular system and enhanced P-selectin expression. Paradoxically, there was marked decrease in dense granule release in response to collagen stimulation in preeclamptic platelets. Platelet microthrombi were detected in circulating whole blood in both HP $(22 \%)$ and PE $(75 \%)$ and not present in controls. Mean blood loss (ml) for caesarean section was $467 \pm 137$ for HP $(n=6)$ and $908 \pm 76$ for $P E(n=6) p<0.01$. There was no difference in blood loss in vaginal deliveries in the 2 groups. No thrombotic events occurred.

Conclusion: The decreased platelet count during pregnancy, accentuated in preeclampsia, and increased blood loss at delivery in preeclampsia were consistent with known literature1. The presence of microthrombi in circulation in both HP and PE groups may account for platelet consumption as a mechanism for low platelets in gestational thrombocytopenia and preeclampsia. The PMD studies identified baseline platelet activation in preeclampsia, but a decreased ability to respond to further stimulation. The free microthrombi in suspension noted in 
peripheral blood in preeclampsia may be the result of sustained platelet activation in preeclampsia. These basally primed platelets then become fatigued and less effective at agonist-stimulated adhesion and aggregation. Further studies are required to correlate these results of PMD with current tests of platelet function, but PMD can potentially utilize the platelet activation process itself to provide biomarkers for staging and phenotyping preeclampsia, and provide prediction for its severity, clinical manifestation and disease progression.

\section{REFERENCES:}

1. Valera MC, Parant $\mathrm{O}$ et al. Physiologic and pathologic changes of platelets in pregnancy. Platelets. 2010; 21:587-595.

2. Agbani EO, Van den Bosch MTJ et al Coofdinated membrane ballooning and procoagulant spreading in human platelets. Circulation. 2015; 132:1414-1424.

3. Agbani EO, Poole AW. Procoagulant plaetlets: generation, function and therapeutic targeting in thrombosis. Blood. 2017; 130:2171.2179.

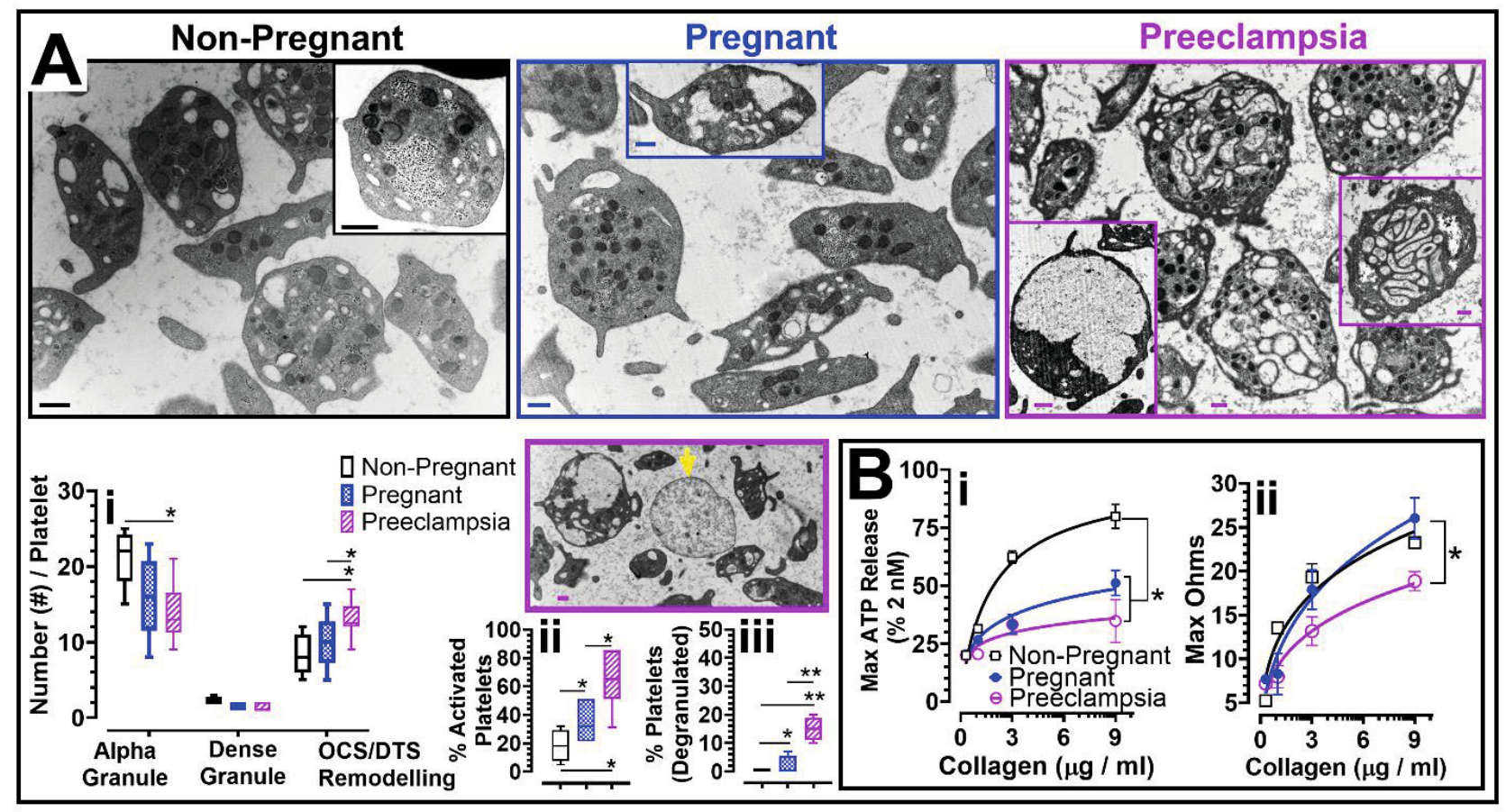


Practice of General Anesthesia for Cesarean Section in a Tertiary Care Center: A Retrospective Cohort Study

Natasha Caissie ${ }^{1}$; Jennifer Héroux ${ }^{1}$; Maxime Lefebvre ${ }^{1}$; Geneviève Rivard ${ }^{1}$; Marie-Chantale Dubois $^{1}$; Frédérick D’Aragon ${ }^{1}$

1 Département d'anesthésiologie et de réanimation, Université de Sherbrooke, Sherbrooke, Québec, Canada

Introduction: In developed countries, 20 to $30 \%$ of births are performed by caesarean section. The gold standard for caesarean section is neuraxial anesthesia, while general anesthesia is reserved for emergencies and failure of or contraindications to neuraxial anesthesia. This has led to a decrease in caesarean sections performed under general anesthesia and the anesthesiologist's exposure to this practice. The lack of evidence-based recommendations for caesarean sections under general anesthesia can lead to significant practice variability and perinatal morbidity, particularly concerning the use of opioids, which can be associated with neonatal respiratory depression. The goal of this study was to describe the practice for caesarean sections performed under general anesthesia at our institution, along with its complications. We also aimed to identify factors that predicted opioid use at anesthesia induction and the need for neonatal resuscitation.

Methods: Ethics approval was obtained from the local research ethics board. We conducted a retrospective, observational, single-centre cohort study at the Centre Hospitalier Universitaire de Sherbrooke, a tertiary care centre catering to high-risk obstetrics patients. We included all adult parturients who underwent caesarean section under general anesthesia between January $1^{\text {st }} 2012$ and December $31^{\text {st }} 2016$. Patients with spinal anesthesia failure or known intrauterine fetal demise were excluded. We collected data regarding anesthetic medication use, maternal medical history, neonatal resuscitation and anesthetic complications. We used a multivariate logistic regression model to identify predictors of opioid use at induction of anesthesia, as well as predictors of neonatal resuscitation.

Results: We identified 234 patients and 194 were included. Propofol was the main induction agent (97.41\%; $n=188)$ and $98.45 \%$ received neuromuscular blockers $(n=193)$. Opioids were used at induction in 33.5\% ( $n=65)$ of patients. Maternal age $(O R=1.04 ; I C=0.98-1.09, p=0.18)$, body mass index $(\mathrm{OR}=1.03 ; \mathrm{IC}=0.97-1.08, \mathrm{p}=0.31)$ and urgent caesarean section $(\mathrm{OR}=1.35$; $\mathrm{IC}=0.41-4.47, \mathrm{p}=0.63$ ) were not independent predictors of opioid use at induction of anesthesia. Opioid use at induction of anesthesia $(O R=2.00 ; I C=0.78-5.12, p=0.13)$, non-reassuring fetal heart rate tracing $(\mathrm{OR}=0.72 ; \mathrm{IC}=0.29-1.82, \mathrm{p}=0.43)$ and incision-to-delivery time $(\mathrm{OR}=1.02$; $\mathrm{IC}=0.92-1.13, \mathrm{p}=0.70$ ) were not independent predictors of neonatal resuscitation. Low gestational age $(\mathrm{OR}=0.75$; $\mathrm{IC}=0.64-0.87, \mathrm{p}=0.0025)$ was an independent predictor of neonatal resuscitation. The maternal anesthetic complication rate was $0.52 \%(n=1)$.

Conclusion: Rapid-sequence induction without opioids is the common practice for caesarean section under general anesthesia at our institution. No maternal independent factors were predictors of opioid use at induction of anesthesia. This is reassuring because of the risk of complications associated with opioid use in obese patients, or for urgent caesarean section with fetal distress. However, low gestational age was the only independent predictor of neonatal resuscitation. This is coherent with the premature neonate's underdeveloped respiratory 
system, and suggests that opioids could be used safely in other high-risk situations (i.e. severe maternal hypertension).

\section{REFERENCES:}

1. Betrán AP, Merialdi M, Lauer JA, et al. Rates of caesarean section: Analysis of global, regional and national estimates. Paediatric and Perinatal Epidem 2007;21(2) : 98-113.

2. Corri B, James A K, Hershel J. Cesarean delivery in the United Kingdom. Time trends in the General Practice Research Database. Obstet Gynecol 2005; 106 : 151-155

3. Joseph KS, Young DC, Dodds L, et al. Changes in maternal characteristics and obstetric practice and recent increases in primary cesarean delivery. Obstet Gynecol2003; 102(4) : 791-800

4. Menacker F, Hamilton BE. Recent trends in cesarean delivery in the United States. NCHS Data Brief 2010; 35:1-8

5. Bucklin BA, Hawkins JL, Anderson JR, et al Obstetric anesthesia workforce survey: Twenty-year update. Anesthesiology 2005; 103:6455-53

6. Djabatey EA, Barclay PM : Difficult and failed intubation in 3430 obstetric general anesthesics. Anaesthesia; 2009:64 : 1168-1171.

7. Devroe S, Van de Velde M, Rex S. General anesthesia for caesariean section. Curr Opin Anaesthesiol. 2015; 28; 240-6

8. Sumikura $\mathrm{H}$. When was the last time you induced general anesthesia for cesarean section? J Anesth.2015; 29:819-820

9. «OMS | Déclaration de l'OMS sur les taux de césarienne ». WHO. Consulté le 30 janvier 2018. http://www.who.int/reproductivehealth/publications/maternal_perinatal_health/csstatement/fr/

10. McDonnel NJ, Paech MJ, Clavisi OM, Scott KL: Difficult and failed intubation in obstetric anesthesia: an observation study of airway and management of complications associated with general anaesthesia for caesarean section. International Journal of Obstetricc Anesthesia; 2008:17 : 292-297

11. Sumikura H, Niwa $H$, Sat M, Nakamoto T, Asai T, Hagihira S. Rethinking general anesthesia for cesarean section. J Anesth (2016) 30:268-273: 2099-4

12. Hawkins JL. Anesthesia-related maternal mortality. Clin Obstet Gynecol 2003; 46:679-687.

13. Gori F, Pasqualucci A, Corradetti F, Milli M \& Peduto VA. Maternal and neonatal outcome after cesarean section : The impact of anesthesia, The Journal of Maternal-Fetal \& Neonatal Medicine 20 :1, 53-57, DOI : 10.1080/14767050601134645

14. Lesage, S.Cesarean delivery under general anesthesia: Continuing Professional Development. Can J Anesth/J Can Anesth (2014) 61:489-503 DOI 10.1007/s12630-0140125-x

15. Practice Guidelines for Obstetric Anesthesia : An Updated Report by the American Society of Anesthesiologists Task Force on Obstetric Anesthesia and the Society for Obstetric Anesthesia and Perinatology . Anesthesiology 124, $\mathrm{n}^{\circ} 2$ (février 2016)

16. Chestnut D,Wong C A, Tsn LC, Ngan Kee W, Beilin Y, Mhyre J. Chestnut's Obstetric Anesthesia: Principles and Practice, 5th Edition. p.459 
17. Rucklidge M. Up-to-date or out-of-date : does thiopental have a isepifuture in obstetric general anaesthesia ? Int J Obstet Anesth 2013; iscepi22 : 175-8.

18. Meek, T. Traditionnal rapid sequence induction is an outmoded technique for caesarean section and should be modified. Opposed. Int J Obstet Anesth 2006;15:229-32.

19. Tumukunde et al. BMC Anesthesiology (2015) 15:63 DOI 10.1186/s12871-015-0044-6

20. Ngan Kee WD, Khaw KS, Ma KC, et al. Maternal and neonatal effects of remifentanil at induction of general anesthesia for cesarean delivery: a randomized, double-blind, controlled trial. Anesthesiology 2006; 104:14 - 20.

21. Chattopadhyay S, Das A, Pahari S. Fetomaternal outcome in severe preeclamptic women undergoing emergency cesarean section under either general or spinal anesthesia. J Pregnancy. 2014; 2014:325098.

22. Mattingly JE, D'Alessio J, Ramanathan J. Effects of obstetric analgesics and anesthetics on the neonate: a review. Paediatr Drugs. 2003; 5:615-27.

23. Carvalho B, Mirikitani EJ, Lyell D, Evans DA, Druzin M, Riley ET. Neonatal chest wall rigidity following the use of remifentanil for cesarean delivery in a patient with autoimmune hepatitis and thrombocytopenia. Int J Obstet Anesth. 2004; 13:53-6.

24. Draisci G, Valente A, Suppa E, Frassanito L, Pinto R, Meo F, De Sole P, Bossù E, Zanfini BA. Remifentanil for cesarean section under general anesthesia: effects on maternal stress hormone secretion and neonatal well-being: a randomized trial. Int J Obstet Anesth. 2008; $17: 130-6$.

25. Noskova P, Blaha J, Bakhouche H, Kubatova J, Ulrichova J, Marusicova P, Smisek J, Parizek A, Slanar O, Michalek P. Neonatal effect of remifentanil in general anaesthesia for caesarean section:isepia randomized trial. BMC Anesthesiol. 2015 Mar 26; 15 h 38. doi : 10.1186/s12871-015-0020-1. eCollection 2015.

26. Bouattour L, Ben Amar H, Bouali Y, Kolsi K, Gargouri A, Khemakhem K, et al. Maternal and neonatal effects of remifentanil for general anaesthesia for caesarean delivery. Ann Fr Anesth Reanim. 2007; 26(4) : 299-304. doi : 10.1016/j.annfar.2007.01.005

27. Mancuso A, De Vivo A, Giacobbe A, Priola V, Maggio Savasta L, Guzzo M, De Vivo D, Mancuso A. General versus spinal anaesthesia for elective caesarean sections: effects on neonatal short-term outcome. A prospective randomised study. J Matern Fetal Neonatal Med. 2010 Oct; 23(10) : 1114-8.

28. Afolabi BB, Lesi FE: Regional versus general anaesthesia for caesarean section. Cochrane Database Syst Rev. (10) 2012

29. Strouch ZY, Dakik CG, White WD, Habib AS: Anesthetic technique for cesarean delivery and neonatal acid-base status: a retrospective database analysis. International Journal of Obstetric Anesthesia (2015) 24, 22-29.

30. Chestnut D,Wong C A, Tsn LC, Ngan Kee W, Beilin Y, Mhyre J. Chestnut's Obstetric Anesthesia: Principles and Practice, 5th Edition. p. 168

31. Apgar V, James LS. Further observations on the newborn scoring system. Am J Dis Child 1962; 104:419-28

32. Yeh $\mathrm{P}$, Emary K, and Impey L: The relationship between umbilical cord arterial $\mathrm{pH}$ and serious adverse neonatal outcome: analysis of 51,519 consecutive validated samples. BJOG 2012; 119: pp. 824-831

33. Ray, Joel G., Karyn E. Medcalf, et Alison L. Park. « Association of Newborn Apgar Score With Maternal Admission to the Intensive Care Unit ». JAMA Pediatrics 170, $\mathrm{n}^{\circ} 1$ (1 janvier 2016): 88. https://doi.org/10.1001/jamapediatrics.2015.3035. 
34. Goldszmidth,, E. Priciples and practices of obstetric airway management. Anesthesiol Clin 2008; 26:109-25.

35. Schneck H, Scheller M. Acid aspiration prophylaxis and caesarean section. Curr Opin Anasthesiol 200;13:261-65.

36. Chestnut D, Wong C A, Tsn LC, Ngan Kee W, Beilin Y, Mhyre J. Chestnut's Obstetric Anesthesia: Principles and Practice, 5th Edition p. 25-26

37. Robins K, Lyons G. Intraoperative awareness during général anesthesia for cesarean delivery. Anesth Analg 2009; 109:886-90.

38. Desprats R, Dumas JC, Giroux M, Campistron G, Faure F, Teixeira MG, et al. Maternal and umbilical cord concentrations of fentanyl after epidural analgesia for cesarean section. Eur J Obstet Gynecol Reprod Biol. 1991; 42:89-94.

39. Apgar V, The newborn (Apgar) scoring system. Reflections and advice, Pediatric clinics of North America, 1966;13:645-650

40. Gilstrap LC, Hauth JC, Hankins GD, and Beck AW: Second-stage fetal heart rate abnormalities and type of neonatal acidemia. Obstet Gynecol 1987; 70 : pp. 191-195

41. Cyna AM, Andrew M, Emmett RS, et al : Techniques for preventing hypotension during spinal anaesthesia for caesarean section. Cochrane Database Syst Rev 2006; (4) :

CD002251 


\title{
Programmed Intermittent Epidural Bolus for Labor Analgesia: an RCT Comparing Bolus
}

\section{Delivery Speeds of $125 \mathrm{~mL} / \mathrm{h}$ versus $250 \mathrm{~mL} / \mathrm{h}$}

\author{
Yusuke Mazda ${ }^{1}$; Cristian Arzola ${ }^{1}$; Kristi Downey ${ }^{1}$; Juliana Caicedo ${ }^{1}$; Xiang Y. Ye ${ }^{2}$; Jose \\ Carvalho $^{1}$
}

1 Department of Anesthesia and Pain Management, Mount Sinai Hospital, University of Toronto 2 Micare Research Centre, Mount Sinai Hospital, University of Toronto

Introduction: Programmed intermittent epidural bolus (PIEB) provides better analgesia for labor pain than continuous epidural infusion (1). The PIEB technique seems to be associated with high sensory block to ice in many women (2-4). While this finding is not associated with important side effects, it suggests that the technique could be optimized. Previous studies have shown that higher speeds of bolus delivery generate higher pressures in the epidural space (5), however one study failed to correlate bolus delivery speeds to quality of labor analgesia or complications (6).

Objectives: We hypothesized that a PIEB technique programmed to deliver boluses at 125 $\mathrm{mL} / \mathrm{h}$ would produce lower sensory levels than at $250 \mathrm{~mL} / \mathrm{h}$.

Methods: This was a double-blind RCT conducted with institutional REB approval and written informed consent of all participants. We recruited term nulliparous laboring ASA II-III women with singleton pregnancies during first stage of labor. Epidural catheter was inserted at L3/4 interspace as determined by ultrasound. All women received a loading dose of $15 \mathrm{~mL}$ of $0.125 \%$ bupivacaine with $50 \mathrm{mcg}$ fentanyl and achieved VNRS $\leq 1$ (0-10) for pain at 20 minutes. PIEB was used for maintenance with $0.0625 \%$ bupivacaine with fentanyl $2 \mathrm{mcg} / \mathrm{mL}$ with the following settings: PIEB bolus $10 \mathrm{~mL}$ Q 40 minutes; PCEA $5 \mathrm{~mL}$; lockout $10 \mathrm{~min}$; hourly max $30 \mathrm{~mL}$. Manual top ups were administered if required. Women were randomized to receive the PIEB regimen at two different delivery speeds: Group 250 (G250): 250 mL/h; Group 125 (G125): 125 $\mathrm{mL} / \mathrm{h}$. In-charge nurses assessed pain scores, sensory block to ice and motor block hourly. The study was terminated six hours after epidural initiation or when women were fully dilated, whichever came first. Primary outcome was the upper sensory block to ice.

Results: Data from 81 women were analyzed (G250: 39; G125: 42). Incidence of women presenting at least one sensory block assessment $\geq$ T6 was not different (G250 $74.4 \%$ vs. G125 $61.9 \%, p=0.23)$. The incidence of women presenting sensory block $\geq \mathrm{T} 6$ in more than $25 \%$ of the assessments was significantly lower in the G125 (61.5\% vs. $28.6 \%, p=0.003)$. Overall incidence of hypotension was significantly lower in G125 (38.5\% vs. 11.6\%, p=0.006). Quality of analgesia and local anesthetic consumption was similar in both groups.

Conclusion: We could not demonstrate a significant difference in our primary outcome, which was the incidence of any sensory block $\geq T 6$. However, G125 was associated with less episodes of sensory block $\geq$ T6 and also with lower overall incidence of hypotension. These differences, although subtle, may be clinically relevant. Given that there is no difference in quality of analgesia, in keeping with a previous study (6), the use of a PIEB regimen with boluses delivered at $125 \mathrm{~mL} / \mathrm{h}$ may be advantageous. 


\section{REFERENCES:}

1. Xu J, Zhou J, Xiao H, et al. A Systematic Review and Meta-Analysis Comparing Programmed Intermittent Bolus and Continuous Infusion as the Background Infusion for Parturient-Controlled Epidural Analgesia. Sci Rep 2019; 9: 2583

2. Epsztein Kanczuk M, Barrett NM, Arzola C, et al.Programmed Intermittent Epidural Bolus for Labor Analgesia During First Stage of Labor: A Biased-Coin Up-and-Down Sequential Allocation Trial to Determine the Optimum Interval Time Between Boluses of a Fixed Volume of $10 \mathrm{~mL}$ of Bupivacaine 0.0625\% With Fentanyl $2 \mu \mathrm{g} / \mathrm{mL}$. Anesth Analg 2017; 124: 537-41

3. Zakus P, Arzola C, Bittencourt R, et al. Determination of the optimal programmed intermittent epidural bolus volume of bupivacaine $0.0625 \%$ with fentanyl $2 \mu \mathrm{g} \cdot \mathrm{ml}^{-1}$ at a fixed interval of forty minutes: a biased coin up-and-down sequential allocation trial. Anaesthesia 2018; 73: 459-65

4. Bittencourt R, Arzola C, Zakus P, et al. A biased coin up-and-down sequential allocation trial to determine the optimum programmed intermittent epidural bolus time interval between 5 $\mathrm{mL}$ boluses of bupivacaine $0.125 \%$ with fentanyl $2 \mu \mathrm{g} \cdot \mathrm{mL}^{-1}$. Can J Anaesth 2019; 66: 1075-81 5. Cardoso MM, Carvalho JC. Epidural pressures and spread of $2 \%$ lidocaine in the epidural space: influence of volume and speed of injection of the local anesthetic solution. Reg Anesth Pain Med 1998; 23: 14-9

6. Lange EMS, Wong CA, Fitzgerald PC et al. Effect of Epidural Infusion Bolus Delivery Rate on the Duration of Labor Analgesia: A Randomized Clinical Trial. Anesthesiology 2018; 128 : 745-53 


\section{Recall of Information Regarding Labour Epidurals: A Survey}

Xiao Xu Chen ${ }^{1}$; Namrta Yashpal ${ }^{1}$; Ilana Sebbag ${ }^{1}$; Indu Singh ${ }^{1}$

1 Department of Anesthesia \& Perioperative Medicine, University of Western Ontario, London, Canada

Introduction: Anesthesiologists are required to procure informed consent from women requesting labour epidural analgesia. However, at least $25 \%$ of women cannot recall a significant amount of information provided to them during informed consent. ${ }^{1,2}$ Being appropriately informed is an important element in facilitating patient involvement in the decision making process and creating a positive birthing experience. ${ }^{3,4}$ This is especially significant as negative birth experiences have been associated with outcomes spanning from postpartum depression to post-traumatic stress disorder. ${ }^{5,6}$ In our academic obstetric center, informed consent is obtained verbally by the anesthesiologist prior to the provision of epidural analgesia. We plan to assess our patients' recall of the information provided and their satisfaction.

Methods: Ethics approval was obtained from the local REB, and formal written informed consent was waived. Between January and March of 2020, postpartum women within 24-48 hours from delivery were asked to participate in this survey (Figure 1). The 10-item questionnaire was created based on a review of the Canadian Medical Protective Association (CMPA) guidelines on informed consent ${ }^{7}$ and previous studies regarding recall of information by obstetric patients. ${ }^{1,8}$ Our primary outcome would be the degree of recall, as measured by proportion of total information provided about labour epidurals. Secondary outcomes include satisfaction with neuraxial labour analgesia, expectation of peripartum pain relief and factors influencing degree of recall and satisfaction.

Results: We aim to enrol 200 postpartum women. Preliminary results $(n=95)$ demonstrate that $55.7 \%$ of respondents received a labour epidural in the past, and $88.4 \%$ had been given information about labour epidurals before admission. Patients reported that they received the best quality information from the obstetrician before labour started. However, the anesthesiologist became a more valuable resource during labour. While $79.7 \%$ of patients subjectively reported recalling all or most of the information provided, only $54.2 \%$ of respondents recalled $75 \%$ or more of the potential side effects discussed. $88.4 \%$ of women felt their epidurals worked as expected or better, and $87.4 \%$ were satisfied with their labour analgesia.

Conclusion: Our preliminary data seems to demonstrate that both obstetricians and anesthesiologists are valued sources of information. Perception of recall was higher than actual recall. Thus, there may be a role for the provision of written information in order to improve the understanding of risks and recall. (Final results will be presented the conference.)

\section{REFERENCES:}

1. Brinkler R et al. Anaesth 2019;74(9):1101-1111.

2. Mahomed K et al. J Obst Gynaecol 2015;35(8):807-809.

3. Rodríguez-Almagro J, Hernández-Martínez A, Rodríguez-Almagro D, Quirós-García J, Martínez-Galiano J, Gómez-Salgado J. Women's Perceptions of Living a Traumatic 
Childbirth Experience and Factors Related to a Birth Experience. International Journal of Environmental Research and Public Health. 2019;16(9):1654.

4. Hodnette E. Pain and women's satisfaction with the experience of childbirth: A systematic review. American Journal of Obstetrics and Gynecology. 2002;186(5):S160-S172.

5. Ayers S, Bond R, Bertullies S, Wijma K. The aetiology of post-traumatic stress following childbirth: a meta-analysis and theoretical framework. Psychological Medicine. 2016;46(6):1121-1134.

6. Bell A, Andersson E. The birth experience and women's postnatal depression: A systematic review. Midwifery. 2016;39:112-123.

7. Evans K. CMPA - Consent: A guide for Canadian physicians. Cmpa-acpm.ca 2016. Available from: https://www.cmpa-acpm.ca/en/advice-publications/handbooks/consent-aguide-for-canadian-physicians

8. Yurashevich M et al. Anaesth. 2019;74(9):1112-1120. 
Figure 1.

\section{Recall of Information Regarding Labour Epidurals: A Survey}

1. Have you received an epidural for labour prior to this delivery?
a. Yes
b. No

2. Were you given information regarding epidural pain relief for this pregnancy before coming in to hospital for your delivery?
a. Yes
b. No

3. Who gave you information regarding epidural pain relief? (select all that apply)
a. Obstetrician
d. Midwife
g. Nurse
b. Anesthesiologist
e. Prenatal classes
h. Online resources
c. Family/friends
f. Other (please expand):

4. Who from the above list provided the best information?
a. Before labour:
b. During labour:

5. Before your epidural, did the anesthesiologist explain: (Select all that apply)
a. The benefits
c. How well it works for pain relief
b. The risks/side effects
d. Alternatives for pain relief (i.e. laughing gas)

6. Of the information listed above, how much do you remember?



7. Which of the following risks do you recall from your discussion with the anesthesiologist? (Select all that apply)
a. Headache
e. Low blood pressure/high block
b. Nerve injury
f. Bleeding/bruising
c. Infection
g. Temporary leg weakness
d. Epidural failure
h. Freezing toxicity (i.e. seizures)

8. Did the epidural work as you expected it to?

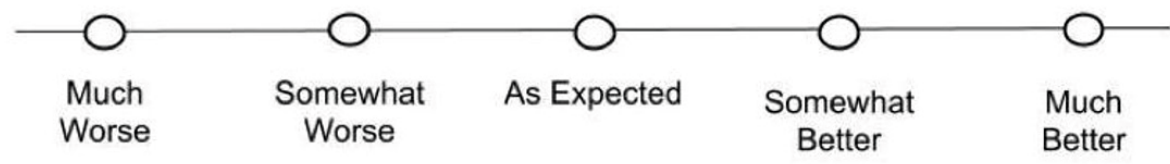

9. Are you satisfied with your labour analgesia?



10. What would help improve your satisfaction with labour analgesia? 


\section{Sepsis in Pregnancy: Trends in Canada (SePTIC Study)}

Indranil Balki ${ }^{1}$; Leyla Baghirzada ${ }^{2}$; Andrew Walker $^{2}$; Stephen Lapinsky ${ }^{3}$; Mrinalini Balki ${ }^{1,4,5}$

1 Department of Anesthesia, Mount Sinai Hospital, University of Toronto, Toronto, Canada

2 Department of Anesthesiology, Perioperative and Pain Medicine, Cumming School of Medicine, University of Calgary, Calgary, Canada

3 Department of Medicine, Mount Sinai Hospital, University of Toronto, Toronto, Canada

4 Department of Obstetrics and Gynaecology, Mount Sinai Hospital, University of Toronto, Toronto, Canada

5 Lunenfeld Tanenbaum Research Institute, Mount Sinai Health System, Toronto, Canada

Introduction: Maternal sepsis, characterized by dysregulated host response to infection during pregnancy, can lead to adverse outcomes in both mother and baby. There are no prior epidemiological data on maternal sepsis in Canada. The objective of this study was to evaluate the incidence, temporo-regional variation, risk factors, morbidity and mortality due to maternal sepsis in Canada.

Methods: Ethics approval was obtained from the local REB. This was a population-based retrospective cohort study based on the nationwide Discharge Abstract Database compiled by the Canadian Institute for Health Information. All delivery records ( $\geq 20$ weeks gestational age) in Canada (excluding Quebec) between April 1, 2004 and March 31, 2017 and associated hospitalization information (demographics, diagnoses, therapeutic procedures) were identified using International Classification of Diseases-10CA/Canadian Classification of Interventions codes. The primary outcome was incidence of sepsis. The secondary outcomes were risk factors, morbidity (organ failure, ICU admission) and mortality. Data were summarized using descriptive statistics. Associations between risk factors and sepsis were derived using unadjusted Odds Ratios (OR).

Results: There were 4,183 cases of sepsis in 3,653,783 hospitalizations for delivery during the study period, with an incidence of $114(95 \% \mathrm{Cl}: 111,118)$ per 100,000 hospitalizations and a mortality rate of $0.5 \%$. There was a trend towards decreasing sepsis rates from 2004 [160 per $100,000$ (95\% Cl: 146, 177)] to 2016 [104 $(93,117)](p<0.001)$. The highest sepsis rate was observed in the Territories [224 per 100,000 (95\% Cl: 167, 301)], while the lowest was in New Brunswick $[77(61,98))]$. Puerperal sepsis was the leading diagnostic code associated with sepsis [72\%, (Table 1)]. Severe sepsis was seen in $14 \%(n=568)$ of all patients with sepsis, which we defined as patients with one or more of: septic shock $(15 \%, n=85)$, organ failure $(61 \%, n=345)$, ICU admission $(78 \%, n=443)$ or mortality $(3 \%, n=19)$. The leading systems involved in organ failure were cardiovascular (41\%) and respiratory (22\%). Among patients with organ failure, $30 \%(n=105)$ had multi-organ system failure. Extremes of maternal age $[<25$ years, OR: 1.59 (95\% Cl: 1.48, 1.71); >40 years, OR: $1.42(1.22,1.65)]$, multiple gestation [2.86 $(2.46,3.32)]$, stillbirths [8.79 $(7.60,10.17)]$, cesarean delivery [3.32 (3.10 to 3.54)], retained products of conception [2.13 (1.69 to 2.69)], postpartum hemorrhage [3.94 (3.65 to 4.24)] and hysterectomy $[24.23(18.16,32.32)]$ were associated with maternal sepsis.

Discussion: Maternal sepsis rates have been decreasing in Canada but remain higher than those observed in the UK and US., ${ }^{1,2}$ Our study shows 1 in 8 women with sepsis develop severe sepsis-related morbidity, which warrants risk stratification, changes in practice guidelines and national preventive strategies. 
REFERENCES:

1. Acosta et al. Severe Maternal Sepsis in the UK, 2011-2012: A National Case Control Study. PLoS Med. 2014;11:e1001672

2. Hensley et al. Incidence of Maternal Sepsis and Sepsis-Related Maternal Deaths in the United States. JAMA. 2019;322:890

Table 1: Morbidity and Mortality of Maternal Sepsis in Canada

\begin{tabular}{|c|c|c|c|c|c|}
\hline Condition & N (\%) & $\begin{array}{c}\text { Incidence }(95 \% \mathrm{CI}) \\
100,000 \text { hospitalizations }\end{array}$ & $\begin{array}{l}\text { Organ failure } \\
\text { N (\%) }\end{array}$ & $\begin{array}{c}\text { ICU Admissions } \\
\text { N (\%) }\end{array}$ & $\begin{array}{l}\text { Mortality } \\
\text { N (\%) }\end{array}$ \\
\hline Puerperal Sepsis & $2990(71.5)$ & $81.8(79.0$ to 84.8$)$ & $165(34.3)$ & $249(56.2)$ & $9(47.4)$ \\
\hline Other Infection During Labor Includes Sepsis & $683(16.3)$ & $18.7(17.3$ to 20.2$)$ & $43(8.9)$ & $48(10.8)$ & $2(10.5)$ \\
\hline Other Sepsis & $441(10.5)$ & $12.1(11.0$ to 13.3$)$ & $139(28.9)$ & $154(34.8)$ & $10(52.6)$ \\
\hline Streptococcal Sepsis & $136(3.2)$ & $3.7(3.2$ to 4.4$)$ & $21(4.4)$ & $23(5.2)$ & 0 \\
\hline Bacteremia & $118(2.8)$ & $3.2(2.7$ to 3.9$)$ & $12(2.5)$ & $9(2.0)$ & $1(5.3)$ \\
\hline $\begin{array}{l}\text { Infection Following Transfusion, Infusion and } \\
\text { Therapeutic Injection }\end{array}$ & $29(0.7)$ & $0.8(0.6$ to 1.1$)$ & $2(0.4)$ & $6(1.4)$ & 0 \\
\hline Necrotizing Fascitis & $23(0.5)$ & $0.6(0.4$ to 1.0$)$ & $6(1.2)$ & $16(3.6)$ & $1(5.3)$ \\
\hline $\begin{array}{l}\text { Systemic Inflammatory Response with Organ } \\
\text { Failure (Includes Severe Sepsis) }\end{array}$ & $7(0.2)$ & $0.2(<0.1$ to 0.4$)$ & $5(1.0)$ & $5(1.1)$ & $2(10.5)$ \\
\hline Toxic Shock Syndrome & $4(0.1)$ & $0.1(<0.1$ to 0.3$)$ & $3(0.6)$ & $2(0.5)$ & 0 \\
\hline Infection from External Stroma of Urinary Tract & $2(<0.1)$ & $<0.1(<0.1$ to 0.2$)$ & 0 & 0 & 0 \\
\hline Salmonella Sepsis & $2(<0.1)$ & $<0.1(<0.1$ to 0.2$)$ & 0 & $1(0.2)$ & 0 \\
\hline Gas Gangrene & $1(<0.1)$ & $<0.1(0$ to 0.2$)$ & 0 & 0 & 0 \\
\hline $\begin{array}{l}\text { Hospitalizations having at least } 1 \text { Sepsis } \\
\text { Condition }\end{array}$ & 4183 & $114.5(111.1$ to 118.0$)$ & $345^{*}$ & 443 & 19 \\
\hline $\begin{array}{l}\text { Hospitalization with more than } 1 \text { Sepsis } \\
\text { Condition }\end{array}$ & 308 & $8.4(7.5$ to 9.4$)$ & 107 & 115 & 7 \\
\hline
\end{tabular}

*345 hospitalizations with at least I organ failure, 481 total organ failures as 105 hospitalizations were associated with 2 or more organ failures. \# 


\section{The Effects of Height and Weight Adjusted Dose of Local Anesthetic Compared to Standard Arbitrary Dosing for Spinal Anesthesia in Elective Cesarean Delivery}

Derek Paradiso Shaw ${ }^{1}$; Yamini Subramani ${ }^{1}$; Yves Bureau ${ }^{2}$; Shalini Dhir ${ }^{1}$

1 Department of Anesthesia and Perioperative Medicine, Western University, London, Ontario, Canada

2 Lawson Health Research Institute, Western University, London, Ontario, Canada

Introduction: Spinal anesthesia is a common anesthetic technique for elective cesarean delivery (CD). A challenge anesthesiologists face is selecting a dose that provides adequate anesthesia to the parturient whilst minimizing harmful side effects. Our primary concern regarding side effects is hypotension as an effect of the spinal anesthetic as it may have harmful effects to both mother and fetus. ${ }^{1}$ Height and weight dependent dose adjustment have been studied in the past. ${ }^{2}$ We hypothesized that a simplified dosing regimen of $0.75 \%$ hyperbaric bupivacaine would provide adequate surgical anesthesia for elective CD while decreasing the incidence of maternal hypotension and the use of vasopressors.

Methods: Ethics approval was obtained from the local REB. In this single centered, double blinded, randomized controlled trial, term parturients ( $n=170$, ASA II, age 18-40, singleton, uncomplicated pregnancy) undergoing elective CD under spinal anesthesia were randomly allocated to receive either a fixed dose regime $(1.6 \mathrm{~mL}$ or $12 \mathrm{mg} 0.75 \%$ hyperbaric bupivacaine) or a height and weight adjusted dose regime (Table 1) along with fentanyl $15 \mathrm{mcg}$ and preservative free morphine $100 \mathrm{mcg}$ intrathecally. Systolic blood pressure of $<90 \mathrm{~mm} \mathrm{Hg}$ or $>25 \%$ decrease from baseline was defined as hypotension. Phenylephrine at interval doses of 100 mcg were given as needed. The primary outcome included maternal hypotension needing phenylephrine (1st line) or ephedrine (2nd line). Patient satisfaction, nausea, vomiting, pruritus, time to adequate surgical anesthesia and conversion to general anesthetic were secondary outcomes. Height and weight parameters were restricted between $150-180 \mathrm{~cm}$ and $50-110 \mathrm{~kg}$, respectively.

Results: Characteristics between the adjusted $(n=86)$ and fixed dose $(n=84)$ groups were similar. The adjusted group experienced less hypotension needing statistically significant lower doses of phenylephrine ( $295 \pm 248 \mathrm{mcg}$ vs $697 \pm 373 ; p 0.000)$ and ephedrine $(1.3 \pm 3.3$ vs $5.5 \pm 13.5 \mathrm{mg} ; \mathrm{p} 0.008)$. The adjusted group experienced statistically significant reductions in nausea and vomiting. There was no difference in overall patient satisfaction, pruritus, and time to adequate surgical anesthesia (Table 2). In the adjusted arm, there was one conversion to general anesthesia.

Conclusion: Height and weight adjusted dose of bupivacaine provided adequate anesthesia and minimized maternal hypotension requiring vasopressor intervention. Additionally, there was significant reduction in nausea and vomiting, which are distressing side effects for the parturient. 


\section{REFERENCES:}

1. Corke B.C., Datta S., Ostheimer G.W., et al. Spinal anaesthesia for Caesarean section. The influence of hypotension on neonatal outcome. Anaesthesia 1982; 37:658-62.

2. Harten J.M., Boyne I, Hannah P. et al. Effects of a height and weight adjusted dose of local anaesthetic for spinal anaesthesia for elective Caesarean Section. Anaesthesia. 2005;

60:348-353. 
Table 1: Height and Weight Adjusted $0.75 \%$ Hyperbaric bupivacaine; shown in volume

\begin{tabular}{|l|l|l|l|l|l|l|l|}
\hline Height (cm) & 150 & 155 & 160 & 165 & 170 & 175 & 180 \\
\hline Weight (kg) & & & & & & \\
\hline 50 & 1.2 & 1.3 & & & & & \\
\hline 60 & 1.1 & 1.2 & 1.3 & 1.4 & & & \\
\hline 70 & 1.1 & 1.1 & 1.3 & 1.3 & 1.4 & 1.5 & \\
\hline 80 & & & 1.2 & 1.3 & 1.4 & 1.5 & 1.6 \\
\hline 90 & & & 1.1 & 1.3 & 1.3 & 1.4 & 1.5 \\
\hline 100 & & & 1.1 & 1.2 & 1.3 & 1.4 & 1.5 \\
\hline 110 & & & & 1.1 & 1.2 & 1.3 & 1.5 \\
\hline
\end{tabular}

Table 2: Primary and Secondary Outcomes Data

\begin{tabular}{|c|c|c|c|c|}
\hline & $\begin{array}{l}\text { Fixed Dose Group } \\
\qquad(\mathrm{n}=84)\end{array}$ & $\begin{array}{l}\text { Adjusted Dose Group } \\
\qquad(n=86)\end{array}$ & $\begin{array}{c}\text { 95\% Cl: } \\
\text { lower, upper }\end{array}$ & P value \\
\hline \multicolumn{5}{|l|}{ Primary Outcome } \\
\hline Phenylephrine (mcg) & $697(373)$ & $295(248)$ & 306,497 & 0.000 \\
\hline Ephedrine (mg) & $5.45(13.48)$ & $1.31(3.32)$ & 1,7 & 0.008 \\
\hline \multicolumn{5}{|l|}{ Secondary Outcomes } \\
\hline Nausea (yes) & 46 & 27 & & 0.002 \\
\hline Vomiting (yes) & 4 & 0 & & 0.041 \\
\hline Pruritus (yes) & 13 & 14 & & 0.913 \\
\hline $\begin{array}{l}\text { Patient Satisfaction } \\
\text { D/N/S/CS }\end{array}$ & $0 / 1 / 33 / 49$ & $2 / 4 / 31 / 49$ & & 0.282 \\
\hline
\end{tabular}




\section{PAIN MANAGEMENT}

\section{HTX-011 Combined with Multimodal Analgesia as Pain Management after Total Knee} Arthroplasty: Results from a Phase 3B Open-Label Study

Scott Hacker ${ }^{1}$; Jia Hu²; Chris Storgard ${ }^{2}$; Pamela Hawn²; Richard Berkowitz ${ }^{3}$

1 Grossmont Orthopedic Medical Group, La Mesa, CA, USA

2 Heron Therapeutics, Inc., San Diego, CA, USA

3 University Orthopedic and Joint Replacement Center, Tamarac, FL, USA

Introduction: Opioid medications are commonly prescribed for pain control following orthopedic surgery, but they can be associated with side effects, poor patient outcomes, and the potential for dependence and misuse..$^{1-3}$

HTX-011 is a novel, extended-release, dual-acting local anesthetic (DALA) containing bupivacaine and low-dose meloxicam in a proprietary polymer allowing for controlled diffusion of active ingredients over 72 hours. In a prior phase 2b study (NCT03015532) in patients undergoing total knee arthroplasty (TKA), HTX-011 (400 mg bupivacaine/12 mg meloxicam) alone reduced pain, decreased opioid use, and reduced time to discharge readiness compared with bupivacaine hydrochloride or saline placebo.

The phase 3b study presented here (NCT03974932) was designed to assess pain control, opioid use, safety, and tolerability of intraoperative HTX-011 + perioperative scheduled nonopioid multimodal analgesia (MMA) in patients undergoing TKA.

Methods: Ethics approval was obtained from the local Research Ethics Board. Before surgery, patients received oral acetaminophen $1 \mathrm{~g}$, celecoxib $200 \mathrm{mg}$, and pregabalin $300 \mathrm{mg}$. All patients underwent TKA with bupivacaine spinal anesthesia and received intraoperative HTX$011400 \mathrm{mg}$ bupivacaine/12 mg meloxicam administered via needle-free periarticular application. For 72 hours following surgery, patients received acetaminophen $1 \mathrm{~g}$ every 8 hours (q8h) and celecoxib $200 \mathrm{mg} \mathrm{q12h} \mathrm{(non-opioid} \mathrm{MMA} \mathrm{regimen).} \mathrm{Opioid} \mathrm{rescue} \mathrm{medication} \mathrm{was}$ administered during the inpatient period only upon patient request. Following discharge at 72 hours, patients were to maintain a scheduled, oral, non-opioid, over-the-counter MMA regimen comprising acetaminophen $1 \mathrm{~g}$ q6h alternating with ibuprofen $600 \mathrm{mg}$ q6h for 4 days. Patients returned for follow-up visits 10 and 28 days after surgery. The primary endpoint was the area under the curve (AUC) of visual analog scale (VAS) scores from 12-48 hours $\left(\mathrm{AUC}_{12-48}\right) .^{4}$

Results: Fifty-one patients undergoing TKA received intraoperative HTX-011 + perioperative non-opioid MMA. Throughout the 72-hour inpatient period, mean pain scores remained in the mild pain range (VAS $<4.4 \mathrm{~cm}$, Figure). ${ }^{5}$ The mean $\mathrm{AUC}_{12-48}$ was 143.2 (SD, 93.5). $57 \%$ of patients never experienced severe pain (VAS $\geq 7.5 \mathrm{~cm}$ ). Median opioid consumption was 22.5 $\mathrm{mg}$ of morphine milligram equivalents per patient through 72 hours ( $<5$ oxycodone $10 \mathrm{mg}$ pills). Most patients (75\%) were discharged without an opioid prescription. The combination of HTX011 with this scheduled non-opioid MMA regimen was well tolerated. 
Conclusion: This phase $3 \mathrm{~b}$ study demonstrated that intraoperative HTX-011 as the foundation of a scheduled non-opioid MMA regimen following TKA has the potential to eliminate severe pain in the majority of patients, maintain mean pain in the mild range, and reduce opioid consumption.

\section{REFERENCES:}

1. Sibia US, et al. J Surg Orthop Adv. 2018;27:231-236.

2. Oderda GM, et al. J Pain Palliat Care Pharmacother. 2013;27:62-70.

3. Hah JM, et al. Anesth Analg. 2017;125:1733-1740.

4. Mont MA, et al. J Arthroplasty. 2018;33:90-96.

5. Jensen MP, et al. J Pain. 2003;4:407-414.

Figure. Mean Pain Intensity Through 72 Hours After TKA as Measured by Visual Analog Scale



MMA, multimodal analgesia; SE, standard error; VAS, visual analog scale. 


\title{
Hydromorphone versus Morphine: A Historical Chart Review to Evaluate the Quality of
} Post-Operative Analgesia

\author{
Shannon Rodrigues ${ }^{1}$; David Shin ${ }^{1}$; Matthew Conway ${ }^{1}$; Stefanie Smulski ${ }^{1}$; Emily Trenker ${ }^{1}$; \\ Harsha Shanthannaa ${ }^{1}$; Thuva Vanniyasingam ${ }^{2}$; Lehana Thabane ${ }^{2}$; James Paul ${ }^{1}$
}

1 Department of Anesthesiology, McMaster University, Health Sciences Centre 2V9, 1280 Main Street West, Hamilton, ON, Canada

2 Department of Health Research Methods, Evidence \& Impact, McMaster University, Health Sciences Centre 2C, 1280 Main Street West, Hamilton, ON, Canada

Introduction: Opioids are the most widely used therapy for pain during the postoperative period. Morphine and hydromorphone are among the most common opioids currently used. ${ }^{1} \mathrm{~A}$ randomized control trial conducted by Shanthanna et al. (2019) compared morphine with hydromorphone for achieving satisfactory analgesia with minimal emesis within 2 hours after surgery. This study found no difference between morphine and hydromorphone regarding analgesia and common side effects. ${ }^{2}$ Our study had a similar primary objective but was conducted as a retrospective cohort study with other differences in study design as well. Our primary objective was to determine if there was a difference in pain score ratings (using a numeric pain rating scale 0 -10) between adult patients receiving intravenous (IV) hydromorphone versus morphine as post-operative analgesia at 2 hours of admission to the post-anesthesia care unit (PACU). Secondary outcomes included proportion of individuals with satisfactory analgesia without substantial postoperative nausea and vomiting (PONV), total equipotent opioid dose, proportion and severity of side effects (PONV, sedation, pruritis), and time until readiness for PACU discharge.

Methods: After ethics board approval, convenience sampling was used to identify the first 605 patients who met inclusion criteria. Patients $>18$ years old and undergoing non-cardiac surgery were identified through the Hamilton Health Sciences (HHS) operating room database. Data extraction from the anesthetic record included patient demographics, surgical procedure, length of procedure, type of anesthetic, and time of extubation. Patients were categorized based on treatment in the PACU with hydromorphone $(n=326)$ or morphine $(n=279)$. PACU flowsheets were used to determine pain scores (from 0-10), nausea/vomiting (scale of 0-3), pruritis (scale of $0-3$ ) sedation (0-4), as well as total opioid dose administered from arrival in PACU until 2 hours or readiness to discharge. Total opioid dose was converted into morphine equivalents using a ratio of 1:5 of hydromorphone: morphine, respectively. 3,4

Results: Regarding the primary outcome of pain reported at 2 hours from admission to PACU, there was no significant difference between hydromorphone and morphine (mean difference: $0.10 ; 95 \% \mathrm{Cl}:-0.21$ to $0.42 ; \mathrm{p}=0.53$ ). Similarly, there were no significant differences between the groups' length of stay in PACU $(p=0.82)$, achieving satisfactory analgesia $(p=0.41)$, incidence of nausea/vomiting $(p=0.08)$, and incidence of sedation $(p=0.36)$. However, a lower equipotent dose of hydromorphone was required for analgesia (mean difference: $-1.35 ; 95 \% \mathrm{Cl}$ : -2.03 to $-0.68 ; p<0.001$ ). There were not enough events to report differences in pruritus and respiratory depression.

Conclusion: There is limited literature comparing morphine to hydromorphone during the postoperative period. This study serves to increase information on prescribing practices, effective analgesia, and associated side effects. Overall, this study found that there is no 
statistically significant difference between the use of IV hydromorphone versus morphine to control pain in the post-operative period.

\section{REFERENCES:}

1. Inturrisi CE. Clinical pharmacology of opioids for pain. Clin J Pain. 2002;18(4 Suppl):S3-13.

2. Shanthanna H, Paul J, Lovrics P, Vanniyasingam T, Devereaux PJ, Bhandari M, et al.

Satisfactory analgesia with minimal emesis in day surgeries: a randomised controlled trial of morphine versus hydromorphone. Br J Anaesth. 2019;122(6):e107-e13.

3. Felden L, Walter C, Harder S, Treede RD, Kayser H, Drover D, et al. Comparative clinical effects of hydromorphone and morphine: a meta-analysis. Br J Anaesth. 2011;107(3):31928.

4. Twycross R, Wilcock A. Palliative Care Formulary. 3rd ed. Nottingham: Palliative Books; 2007. 
Is Intrathecal Analgesia Associated with Reduced Post-Operative Pain in Laparoscopic Liver Resections?

April (YiChen) Liu ${ }^{1}$; Thuva Vanniyasingam²; Antonella Tidy ${ }^{3}$; William Yao ${ }^{1}$; David Shin ${ }^{1}$; Pablo Serrano ${ }^{4}$; Saeda Nair ${ }^{3}$

1 Undergraduate Medicine, Michael G. DeGroote School of Medicine, Hamilton, Canada

2 Biostatistics Unit, St. Joseph's Healthcare, Hamilton, Canada

3 Department of Anesthesiology, Hamilton Health Sciences, Hamilton, Canada

4 Department of Surgery, Hamilton Health Sciences, Hamilton, Canada

Introduction: In an era of Enhanced Recovery After Surgery (ERAS), intrathecal analgesia has been proposed as an alternative to epidural analgesia and patient controlled analgesia (PCA). Intrathecal analgesia have been recommended by the ERAS Society in laparoscopic colon resections and are also used in open liver resections; however, they have not been extensively studied in laparoscopic hepatobiliary surgeries. The primary objective of this study was to explore postoperative pain at 48 hours among patients who underwent laparoscopic liver resections (LLR), receiving either intrathecal analgesia with or without PCA versus PCA alone. Secondary objectives were to determine the association of treatment type with post-operative pain outcomes at 48 hours and surgical complications.

Methods: Ethics approval was obtained from the local REB. Patients who underwent LLRs between January 2016 and April 2019, and had intrathecal analgesia administration and/or PCA were included. To describe postoperative pain, descriptive statistics were completed for both treatment groups for each pain outcome. Postoperative pain outcomes included: cumulative opioid consumption, visual analog scale scores, length of time without requiring opioids, length of time unable to mobilize, and use of non-opioid pain-control medication (regional analgesia, acetaminophen, nonsteroidal anti-inflammatory drugs, gabapentinoids, tramadol, lidocaine, and $\mathrm{N}$-methyl-d-aspartate class of glutamate receptor antagonists). To describe the postoperative pain levels at 48 hours, descriptive statistics were presented by treatment group for each pain outcome. Unadjusted regression analyses were then performed to explore the association of treatment type with each pain and surgical complication outcome. Multivariable linear regression analysis was then conducted to determine other factors associated with increased cumulative postoperative opioid consumption at 48 hours.

Results: Out of 111 patients identified, 79 patients met the inclusion and exclusion criteria; 22 patients had intrathecal analgesia with or without PCA and 57 patients had PCA only. There were no statistically significant differences in baseline characteristics, use of non-opioid pain control, and post-operative complications between the two groups. Intrathecal analgesia use was associated with reduced post-operative opioid consumption, measured in oral morphine equivalents, compared to PCA alone (Mean Difference $(95 \% \mathrm{Cl})=-45.92(-83.10,-8.75)$; $p=0.016)$. In the multivariable regression analysis, it remained significant $(p=0.036)$ along with age $(p=0.005)$. Intrathecal analgesia were also associated with a greater length of time without requiring opioids post-operatively (Mean Difference $(95 \% \mathrm{Cl})=0.75(0.19,1.31) ; p=0.010)$.

Conclusion: Intrathecal analgesia administration has the potential to decrease post-operative opioid use for patients undergoing LLRs. The findings from this study are consistent with the 
ERAS Society recommendations for laparoscopic colorectal surgery and offers support for the safety and efficacy of using intrathecal analgesia in the setting of LLRs for ERAS. This study is limited by its exploratory nature and small sample size; further research is necessary before recommending the use of routine intrathecal analgesia in LLRs.

\section{REFERENCES:}

1. Egger ME, Gottumukkala V, Wilks JA, Soliz J, Ilmer M, Vauthey JN, Conrad C. Anesthetic and operative considerations for laparoscopic liver resection. Surgery. 2017; 161(5):1191-1202. 2. Levy BF, Scott MJ, Fawcett W, Fry C, Rockall TA. Randomized clinical trial of epidural, spinal or patient-controlled analgesia for patients undergoing laparoscopic colorectal surgery. $\mathrm{Br}$ J Surg. 2011; 98(8): 1068-1078.

3. Dichtwald S, Ben-Haim M, Papismedov L, Hazan S, Cattan A, Matot I. Intrathecal morphine versus intravenous opioid administration to impact postoperative analgesia in hepato-pancreatic surgery: a randomized controlled trial. Journal of anesthesia. 2017; 31(2): 237-245.

4. Kasivisvanathan R, Abbassi-Ghadi N, Prout J, Clevenger B, Fusai GK, Mallett SV. A prospective cohort study of intrathecal versus epidural analgesia for patients undergoing hepatic resection. HPB. 2014; 16(8): 768-775.

5. Koea JB, Young Y, Gunn K. Fast track liver resection: the effect of a comprehensive care package and analgesia with single dose intrathecal morphine with gabapentin or continuous epidural analgesia. HPB Surgery. 2009; 2009: 271986.

6. Tzimas P, Prout J, Papadopoulos G, Mallett S. Epidural anaesthesia and analgesia for liver resection. Anaesthesia 2013; 68: 628-635

7. Lee JH, Park JH, Kil HK, Choi SH, Noh SH, Koo BN. Efficacy of intrathecal morphine combined with intravenous analgesia versus thoracic epidural analgesia after gastrectomy. Yonsei medical journal, 2014; 55(4): 1106-1114.

8. Clark CJ, Ali SM, Zaydfudim V, Jacob AK, Nagorney DM. Safety of an enhanced recovery pathway for patients undergoing open hepatic resection. PloS one. 2016; 11(3): e0150782.

9. Melloul E, Hübner M, Scott M, et al. Guidelines for perioperative care for liver surgery: Enhanced Recovery After Surgery (ERAS) society recommendations. World J Surg. 2016; 40(10):2425-40.

10. Dindo D, Demartines N, Clavien PA. Classification of surgical complications: a new proposal with evaluation in a cohort of 6336 patients and results of a survey. Ann Surg. 2004; 240(2):205-213.

11. Ramsay, M. Acute Postoperative Pain Management. Proc (Bayl Univ Med Cent). 2000 Jul; 13(3): 244-247.

12. Roy JD, Massicotte L, Sassine MP, Seal RF, Roy A. A comparison of intrathecal morphine/fentanyl and patient-controlled analgesia with patient-controlled analgesia alone for analgesia after liver resection. Anesth Analg. 2006; 103: 990-994.

13. Kasivisvanathan R, Abbassi-Ghadi N, Prout J, Clevenger B, Fusai GK, Mallett SV. A prospective cohort study of intrathecal versus epidural analgesia for patients undergoing hepatic resection. HPB (Oxford). 2014; 16: 768-775

14. Gustafsson UO, Scott MJ, Hubner M, Nygren J, Demartines N, Francis N et al. Guidelines for Perioperative Care in Elective Colorectal Surgery: Enhanced Recovery After Surgery (ERAS $\otimes)$ Society Recommendations: 2018. World Journal of Surgery. 2019; 43(3): 659-695. 15. Levy BF, Scott MJ, Fawcett WJ, Rockall TA. 23-hour-stay laparoscopic colectomy. Dis Colon Rectum 2009; 52: 1239-1243

16. Cook TM, Counsell D, Wildsmith JA. Major complications of central neuraxial block: report 
on the Third National Audit Project of the Royal College of Anaesthetists. Br J Anaesth. 2009 Feb; 102(2): 179-190

Table 1. Post-operative Pain Outcomes at 48 Hours

\begin{tabular}{|c|c|c|c|}
\hline Postoperative Pain Outcomes & $\begin{array}{l}\text { Intrathecal morphine } \\
\text { with or without PCA } \\
(\mathrm{N}=22)\end{array}$ & $\begin{array}{l}\text { PCA only } \\
(\mathrm{N}=57)\end{array}$ & Estimate $(95 \% \mathrm{CI}) \mathrm{p}$ \\
\hline Continuous outcomes & Mean(sd) & Mean(sd) & Mean Difference $(95 \% \mathrm{CI}) \mathrm{p}$ \\
\hline $\begin{array}{l}\text { Cumulative opioid consumption } \\
\text { within } 48 \text { hours using morphine } \\
\text { equivalents (mg); mean(sd) } \\
\text { Between } 0-24 \text { hours (Day 1) } \\
\text { Between } 24-48 \text { hours (Day 2) }\end{array}$ & $\begin{array}{l}70.61(41.95) \\
27.57(19.99) \\
43.05(30.08) \\
\end{array}$ & $\begin{array}{l}116.54(83.35) \\
70.67(50.05) \\
45.86(49.39)\end{array}$ & $-45.92(-83.10,-8.75) 0.016$ \\
\hline VAS Score at 48 hours; mean (sd) & $3.23(1.93)$ & $2.53(2.05)$ & $-0.02(-0.17,0.12) 0.178^{£}$ \\
\hline & Median (Q1,Q3) & Median (Q1,Q3) & Mean Difference $(95 \% \mathrm{CI}) \mathrm{p}$ \\
\hline $\begin{array}{l}\text { Length of time without requiring } \\
\text { opioid (minutes); median (Q1,Q3) }\end{array}$ & $70.00(45.00,378.00)$ & $39.00(28.00,65.00)$ & $0.75(0.19,1.31) 0.010^{¥}$ \\
\hline $\begin{array}{l}\text { Length of time unable to mobilize } \\
\text { (hours); median (Q1,Q3) } \\
\text { Missing }\end{array}$ & $\begin{array}{l}26.68(21.00,48.78) \\
1\end{array}$ & $\begin{array}{l}24.99(20.51,42.92) \\
1\end{array}$ & $\begin{array}{l}0.07(-0.19,0.32) 0.601^{¥} \\
1\end{array}$ \\
\hline $\begin{array}{l}\text { Non-opioid pain-control } \\
\text { medication }\end{array}$ & $\mathrm{n}(\%)$ & $\mathrm{n}(\%)$ & Odds Ratio $(95 \% \mathrm{CI}) \mathrm{p}$ \\
\hline Acetaminophen; $\mathrm{n}(\%)$ & $14(63.64)$ & $28(49.12)$ & $1.81(0.66,4.97) 0.249$ \\
\hline $\begin{array}{l}\text { Nonsteroidal anti-inflammatory } \\
\text { drugs; } n(\%)\end{array}$ & $3(13.64)$ & $10(17.54)$ & $0.74(0.18,3.00) 0.676$ \\
\hline Gabapentinoids; $n(\%)$ & 0 & $2(3.51)$ & Not enough events \\
\hline
\end{tabular}

${ }^{\text {E} A d j u s t e d ~ f o r ~ P a i n ~ a t ~ P A C U ~ a r r i v a l ~}$

*Based on $\ln$ transformation of outcome

Comment: 0 events for regional analgesia, tramadol, lidocaine, and N-methyl-d-aspartate class of glutamate receptor antagonists. 
Opioid-Free Recovery After Bunionectomy with HTX-011, an Extended-Release Local Anesthetic, in Combination with Non-Opioid Multimodal Analgesia

Jia Hu ${ }^{1}$; Richard A. Pollak²; Chris Storgard ${ }^{1}$; Pamela Hawn ${ }^{1}$; Gwo-Chin Lee ${ }^{3}$

1 Heron Therapeutics, Inc., San Diego, CA, USA

2 Endeavor Clinical Trials, San Antonio, TX, USA

3 Department of Orthopaedic Surgery, University of Pennsylvania, Philadelphia, PA, USA

Introduction: Opioid medications are commonly used for pain management in the postoperative setting, but they can be associated with side effects, poor patient outcomes, and the potential for dependence and misuse. Consequently, innovative and effective non-opioid pain management strategies are needed.

HTX-011, an extended-release, dual-acting local anesthetic (DALA) containing bupivacaine and low-dose meloxicam in a controlled-release polymer, has demonstrated superiority to bupivacaine hydrochloride through 72 hours in multiple surgical models. ${ }^{1-3}$ In a previously conducted phase 3 study in bunionectomy with osteotomy and internal fixation (EPOCH 1), treatment with HTX-011 alone provided superior pain relief, reduced the incidence of severe pain, significantly reduced total opioid consumption and resulted in significantly more opioid-free patients through 72 hours than bupivacaine hydrochloride. ${ }^{1}$

The follow-on study presented here (NCT03718039) was designed to assess the efficacy and safety of HTX-011 as the foundation of a scheduled postoperative non-opioid multimodal analgesia (MMA) regimen in bunionectomy.

Methods: Ethics approval was obtained from the local Research Ethics Board. Patients undergoing bunionectomy with osteotomy and internal fixation using lidocaine Mayo block received intraoperative $\mathrm{HTX}-011$ (up to $2.1 \mathrm{~mL}, \leq 60 \mathrm{mg}$ bupivacaine/1.8 mg meloxicam) via needle-free application. Throughout the 72-hour inpatient postoperative period, patients received a non-opioid MMA regimen of oral ibuprofen $600 \mathrm{mg}$ every 6 hours (q6h) and oral acetaminophen $1 \mathrm{~g}$ q6h (alternating every $3 \mathrm{~h}$ ). Rescue opioids were available upon request. At discharge, patients were to use ibuprofen $600 \mathrm{mg}$ q6h as needed and to add acetaminophen 1 $\mathrm{g}$ q6h if pain persisted. Only patients who received $\geq 10 \mathrm{mg}$ oxycodone $\leq 12$ hours before discharge were to receive an opioid prescription. Key efficacy assessments were pain intensity (on an 11-point [0-10] Numeric Rating Scale [NRS]) and opioid use. Safety assessments included adverse events (AE) and clinical laboratory tests.

Results: Thirty-one patients received HTX-011; all completed the study. Patient baseline characteristics were similar between the initial phase 3 study and this follow-on study. Mean pain intensity remained within the mild range (NRS $\leq 4)$ through 72 hours (Figure). Twenty-four patients $(77 \%)$ required no opioids (ie, were opioid-free) through 72 hours, and all of these patients remained opioid-free through the 28-day recovery period. Only one patient required an opioid prescription at discharge. Twenty patients (65\%) experienced an AE; the most common were nausea (23\%) and vomiting (10\%). No serious AEs were reported and there was no evidence of gastrointestinal, renal, or hepatic toxicity. 
Conclusion: HTX-011, when administered alongside scheduled non-opioid over-the-counter ibuprofen and acetaminophen, resulted in opioid-free recovery in $77 \%$ of patients after bunionectomy. Average pain was maintained in the mild range through 72 hours. The regimen was well tolerated. HTX-011 has the potential to be the foundation of a non-opioid MMA regimen that effectively treats pain and achieves opioid-free recovery.

\section{REFERENCES:}

1. Viscusi E, et al. Reg Anesth Pain Med. 2019;44:700-706.

2. Viscusi E, et al. Hernia. 2019;23;1071-1080.

3. Ottoboni T, et al. Reg Anesth Pain Med. 2019;45;117-123.

Figure. HTX-011 With a Non-Opioid MMA Regimen Maintained Pain Scores in the Mild Range Through the 72-Hour Postoperative Period After Bunionectomy

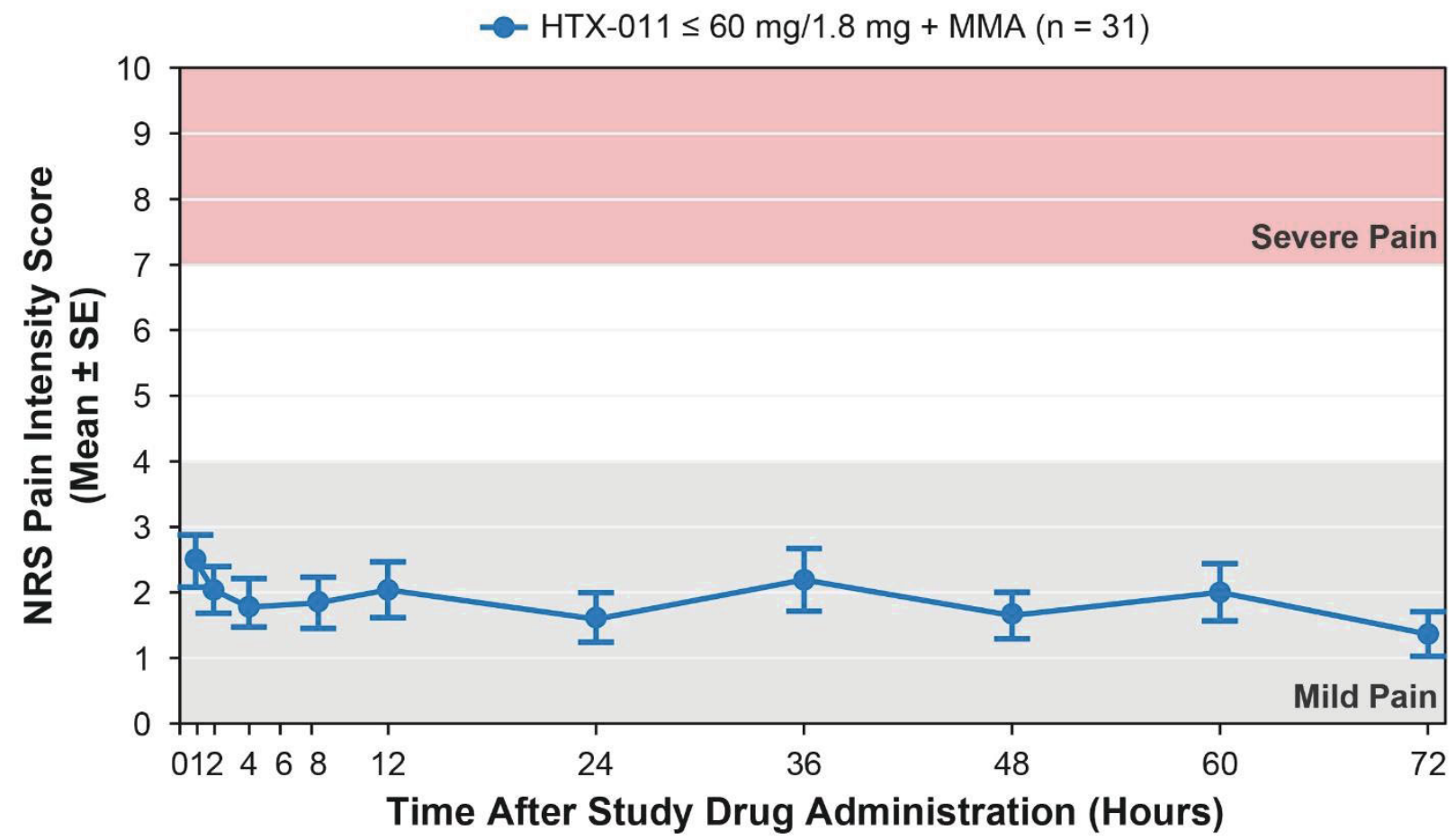

MMA, multimodal analgesia (ibuprofen and acetaminophen); NRS, numeric rating scale of pain intensity; SE, standard error. 
The Effect of Intra-Operative Use of Ketamine and Lidocaine in DIEP Flap Reconstruction on Post Operative Pain

Lina Basri ${ }^{1}$; Shaqayeq Marashi ${ }^{1}$; Kevin Armstrong ${ }^{1}$; Qutaiba Tawfic $^{1}$

1 Department of Anesthesia and Perioperative Medicine, London Health Sciences Centre, Western University, London, Ontario, Canada

Introduction: DIEP flap reconstruction surgery is considered as a major surgery with a substantial post-operative pain ${ }^{1,2}$. The use of multimodal analgesia decreases the postoperative opioid usage and its related side effects ${ }^{2,3}$.

Method: We conducted a descriptive, retrospective study of DIEP flap reconstruction over a period of 5 years (September 2013-September 2018). The study was approved by our institution's Research Ethics Board. We included adult female patients who had no history of a significant chronic opioid usage. Data was obtained from the hospital Electronic Medical Record (EMR) and patients' charts. The collected data included patient's demographic information, duration of surgery, duration of hospitals stay, perioperative analgesic medications/interventions, postoperative pain scores and opioid related side effects. A twotailed Welch's t-test was used for analysis.

Results: During the study period, 106 patients were identified. One patient was excluded for using more than $80 \mathrm{mg}$ of oral morphine equivalent per day. Data of 105 patients $(53.33 \%$ bilateral) were included in the analysis. The mean age of 51.24 years $( \pm 0.90)$ and mean weight $78.98 \mathrm{~kg}( \pm 1.30)$. The mean duration of the surgical procedure was $561.19 \mathrm{~min}( \pm 12.26)$. Post operatively, the 2-hour mean visual analogue scores (VAS) at rest $2.82( \pm 0.22)$ and activity 3.53 $( \pm 0.24)$. The 24 -hour mean visual analogue scores (VAS) at rest $2.86( \pm 0.22)$ and activity 4.13 $( \pm 0.24)$. Intraoperatively, lidocaine infusion $(0.5-2 \mathrm{mg} / \mathrm{kg} / \mathrm{hr})$ was used in 10 patients $(9.52 \%)$, ketamine $(5-12 \mathrm{mg} / \mathrm{hr})$ was used in 32 patients $(30.47 \%)$, both lidocaine and ketamine used in 25 patients $(23.30 \%)$ and opioid only analgesia used 38 patients ( $36.19 \%)$. A two-tailed Welch's t-test was performed to compare post operative VAS these groups at 2 and 24 hours (Table1). The combination of intraoperative lidocaine and ketamine analgesia reduced the 24hour post operative pain during activity when compared to no intraoperative adjuvant analgesia $(p=<0.05)$. The standard post operative analgesia includes opioid-PCA and oral multimodal analgesia. The mean length of hospital stay (days) for the four groups as follow; opioid only $5.75( \pm 0.21)$, ketamine $5.15( \pm 0.16)$, lidocaine $5.6( \pm 0.22)$ and both lidocaine and ketamine 5.16 $( \pm 0.12)$. The use of ketamine and/or a mixture of lidocaine and ketamine, but not lidocaine alone, causes a significant reduction in hospital stay $(p<0.05)$. The use of intraoperative analgesia did not show a significant reduction in first postoperative day opioid related side effects (nausea, vomiting and pruritus) OR 0.9-1.0 (95\% Cl).

Discussion: Our data suggests that the use of a combination of lidocaine and ketamine intraoperatively reduced the 24-hour post-operative pain during activity in this group of patients. More prospective, well designed studies are needed to determine the effect of intra-operative lidocaine and ketamine on acute post-DIEP flap pain. 


\section{REFERENCES:}

1. Skraastad BK, Knudsen C, Jackson C, Utheim TP, Pripp AH, Tønseth KA. Quality of life, patient satisfaction and cosmetic outcome after delayed breast reconstruction using DIEP flap: a 10 years' follow-up survey. J Plast Surg Hand Surg. 2019 Jan 18:1-6

2. Bar-Meir ED, Yueh JH, Hess PE, Hartmann CE, Maia M, Tobias AM, Lee BT. Postoperative Pain Management in DIEP Flap Breast Reconstruction: Identification of Patients With Poor Pain Control. Eplasty. 2010 ;10. pii: e59.

3. Kulkarni AR, Pusic AL, Hamill JB, Kim HM, Qi J, Wilkins EG, Roth RS.Factors Associated with Acute Postoperative Pain Following Breast Reconstruction. JPRAS Open. 2017 ;11:113.

\begin{tabular}{|c|c|c|c|c|c|c|}
\hline \multicolumn{4}{|c|}{ 2-hour VAS (rest) } & \multicolumn{3}{|c|}{ 2-hour VAS (Activity) } \\
\hline $\begin{array}{l}\text { Adjuvant } \\
\text { analgesia }\end{array}$ & Mean & Variance & $P$ value & Mean & Variance & P value \\
\hline Lidocaine & 2.6 & 2.93 & 0.61 & 3.9 & 5.87 & 0.83 \\
\hline Ketamine & 2.78 & 4.75 & 0.76 & 3.43 & 5.60 & 0.64 \\
\hline $\begin{array}{l}\text { Lidocaine } \\
\text { and } \\
\text { ketamine }\end{array}$ & 2.80 & 6.75 & 0.83 & 3.48 & 7.09 & 0.73 \\
\hline None & 2.94 & 6.32 & & 3.71 & 6.80 & \\
\hline \multicolumn{4}{|c|}{ 24-hour VAS (rest) } & \multicolumn{3}{|c|}{ 24-hour VAS (Activitv) } \\
\hline $\begin{array}{l}\text { Adjuvant } \\
\text { analgesia }\end{array}$ & Mean & Variance & $P$ value & Mean & Variance & $P$ value \\
\hline Lidocaine & 3.50 & 2.27 & 0.44 & 4.70 & 2.67 & 0.95 \\
\hline Ketamine & 2.81 & 6.15 & 0.70 & 3.87 & 5.01 & 0.13 \\
\hline $\begin{array}{l}\text { Lidocaine } \\
\text { and } \\
\text { ketamine }\end{array}$ & 2.48 & 5.26 & 0.35 & 3.32 & 8.06 & 0.048 \\
\hline None & 3.02 & 5.10 & & 4.73 & 6.36 & \\
\hline
\end{tabular}

Table 1: The comparison between the visual analogue scores of the use of adjuvant analgesics with opioid only analgesia by using Welch's t-test. 


\section{Understanding Opioid Prescribing versus Opioid Consumption in the Postoperative} Period

Ekta Khemani ${ }^{1}$; Michael Szpejda ${ }^{2}$; Maham Khalid ${ }^{3}$; Sanjho Srikandarajah ${ }^{2}$; Tasnim Zaman ${ }^{3}$; Ian Brown $^{4}$

1 Department of Anesthesia, McMaster University, Hamilton, Canada

2 Department of Anesthesiology and Pain Management, University of Toronto, Toronto, Canada

3 Michael G. DeGroote School of Medicine, McMaster University, Hamilton, Canada

4 Department of Surgery, Niagara Health System, Saint Catharines, Canada

Introduction: In the past twenty-five years, opioid related deaths have increased four-fold in Ontario, and opioid related emergency department visits have tripled between 2008 and 2016 in the Niagara region alone. Despite vast media coverage of the opioid crisis, opioid prescriptions continue to rise. Indeed, a significant number of overdose deaths have been linked to opioid prescriptions in the postoperative period. There is great variability in post-surgical opioid prescriptions across procedures at the time of discharge. Given that overprescribing of narcotics by physicians could further contribute to the opioid crisis, it is timely to understand opioid prescribing in the postoperative setting. This study aims to improve opioid prescription strategies postoperatively at the Niagara Health System (NHS) by identifying and, ultimately, mitigating the discrepancy between the amount of opioids prescribed by physicians and the proportion of prescribed opioids consumed by patients.

Methods: Ethics approval was obtained from the local REB. This prospective study included patients undergoing one of eighteen elective, day surgeries across eight surgical specialities, which had been examined for opioid overprescribing practices in the literature. Exclusion criteria included patients $<18$ years of age, non-English speaking, pregnant, or those with a history of opioid use or chronic pain. Patients from all three NHS sites (Welland Hospital, Greater Niagara General Hospital, St. Catharine's General Hospital) were included. Participants who provided consent were given a questionnaire to establish pre-operative prescription use, and copies of discharge opioid prescriptions were obtained from chart retrieval. Patients were contacted by phone on post-operative day ten to fourteen to establish the amount of postoperative narcotic prescription consumed. If they could not be reached after three attempts, they were deemed lost to follow-up. Data was collected from March - June 2019.

Results: A total of 255 patients, from the eighteen surgeries included in the study, consented to the study and met inclusion criteria. For simplicity, all opioid prescriptions were converted to morphine equivalents (ME) for data analysis. It was found that on average, only $35.7 \%+19 \%$ opioids prescribed were used postoperatively to achieve adequate pain control (see Figure 1).

Conclusion: Our results demonstrate that patients across multiple surgical specialties are overprescribed narcotics in the elective, day surgery postoperative setting. This is one of the first studies in Canada to examine opioid prescribing habits in conjunction with patient use on a large scale, though our results echo what has been found in the American literature. Following the results of our study, multidisciplinary education sessions were held to disseminate information and gather feedback from all stakeholders. Patient education handouts on multimodal analgesia, appropriate opioid use, and opioid disposal have been implemented following the results of the study. Perioperative order sets are also being changed to reduce overprescribing in the postoperative period. We will continue to monitor the use of postoperative 
opioid prescribing for elective day surgery patients, with the intent of minimizing opioid overprescribing.

\section{REFERENCES:}

1. https://odprn.ca/wp-content/uploads/2018/08/Infographic-Opioid-Prescriptions-Deaths.png

2. https://www.publichealthontario.ca/en/data-and-analysis/substance-use/interactive-opioidtool

3. http://opioidprescribing.hqontario.ca/

4. Hill MV, McMahon ML, Stucke RS, Barth RJ Jr. Wide Variation and Excessive Dosage of Opioid Prescriptions for Common General Surgical Procedures. Ann Surg. 2017 Apr;265(4):709-714. doi: 10.1097/SLA.0000000000001993. PubMed PMID: 27631771.

5. Thiels CA, Ubl DS, Yost KJ, Dowdy SC, Mabry TM, Gazelka HM, Cima RR, Habermann EB. Results of a Prospective, Multicenter Initiative Aimed at Developing Opioid-prescribing Guidelines After Surgery. Ann Surg. 2018






\title{
PEDIATRIC
}

\section{Consent Practices Among Canadian Pediatric Anesthesiologists}

\author{
Tyeren Deacon ${ }^{1}$; Joanna Dion ${ }^{2}$
}

1 Medical Student, Queen's University, Kingston, Canada

2 Perioperative Medicine, Kingston General Hospital, Kingston, Canada

Introduction: Currently, there is little to no research in the area of consent for pediatric anesthesia. We aim to clarify the current consent practices of pediatric anesthesiologists in Canada. Physicians best modify their practice (if required) when the current state of practice is clearly outlined. With this data, we can show similarities or differences with how our consent process aligns with the preferences of parents and guardians of children undergoing anesthesia. This may be a starting point for suggestions for a standardized patient-centred consent discussion.

Methods: Ethics approval was obtained from the local REB. This study survey was sent out via email invitation to members of the Canadian Pediatric Anesthesia Society (CPAS) which comprises of pediatric anesthesiologists practicing at children's hospitals across Canada. This study is web-based and consists of 14 multiple choice questions with content including practitioner demographics (ie. years in practice), patient demographics (ie. age of patients), and components of the consent process for that practitioner (ie. risks and incidence mentioned to patients for informed consent). The Queen's survey tool Qualtrics was used to design and distribute the survey. The survey was sent out on August 11th, 2019 and the survey closed to respondents November 4th, 2019. It was subsequently analyzed by the Principal Investigator and the Co-investigator.

Results: The survey was completed by 60 CPAS Members (response rate TBD). The vast majority of the respondents were Anesthesiologists (96.7\%), 98.3\% of them have done a fellowship in Pediatric Anesthesia. The 5 most commonly discussed risks for general anesthesia were PONV (86.1\%), sore throat $(75.5 \%)$, dental damage (66.8\%), emergence delirium (54.5\%), and respiratory concerns (45.7\%). The most commonly discussed risks for regional anesthesia were infection (84.3\%), inadequate anesthesia (82.6\%), minor nerve damage $(75.5 \%)$, major nerve damage $(73.7 \%)$, and hematoma $(73.7 \%)$. For sedation the most commonly discussed risks were conversion to general anesthesia $(72.2 \%)$, awareness $(47.6 \%)$, respiratory concern $(37.0 \%)$, paradoxical response $(35.2 \%)$, and allergy $(19.4 \%)$. Other notable results found that $46.6 \%$ of respondents obtained consent for the administration of rectal acetaminophen, and that the most commonly volunteered age for consent was 14 years old. It was also found that the majority of practitioners gain verbal consent $(61.0 \%)$ as opposed to written $(8.5 \%)$ or both $(30.5 \%)$.

Conclusion: Results show that the risks discussed in the consent process for pediatric anesthesia vary largely among practitioners. More research is needed to determine if there is a need for a standardized model for patient-centered consent discussion. 


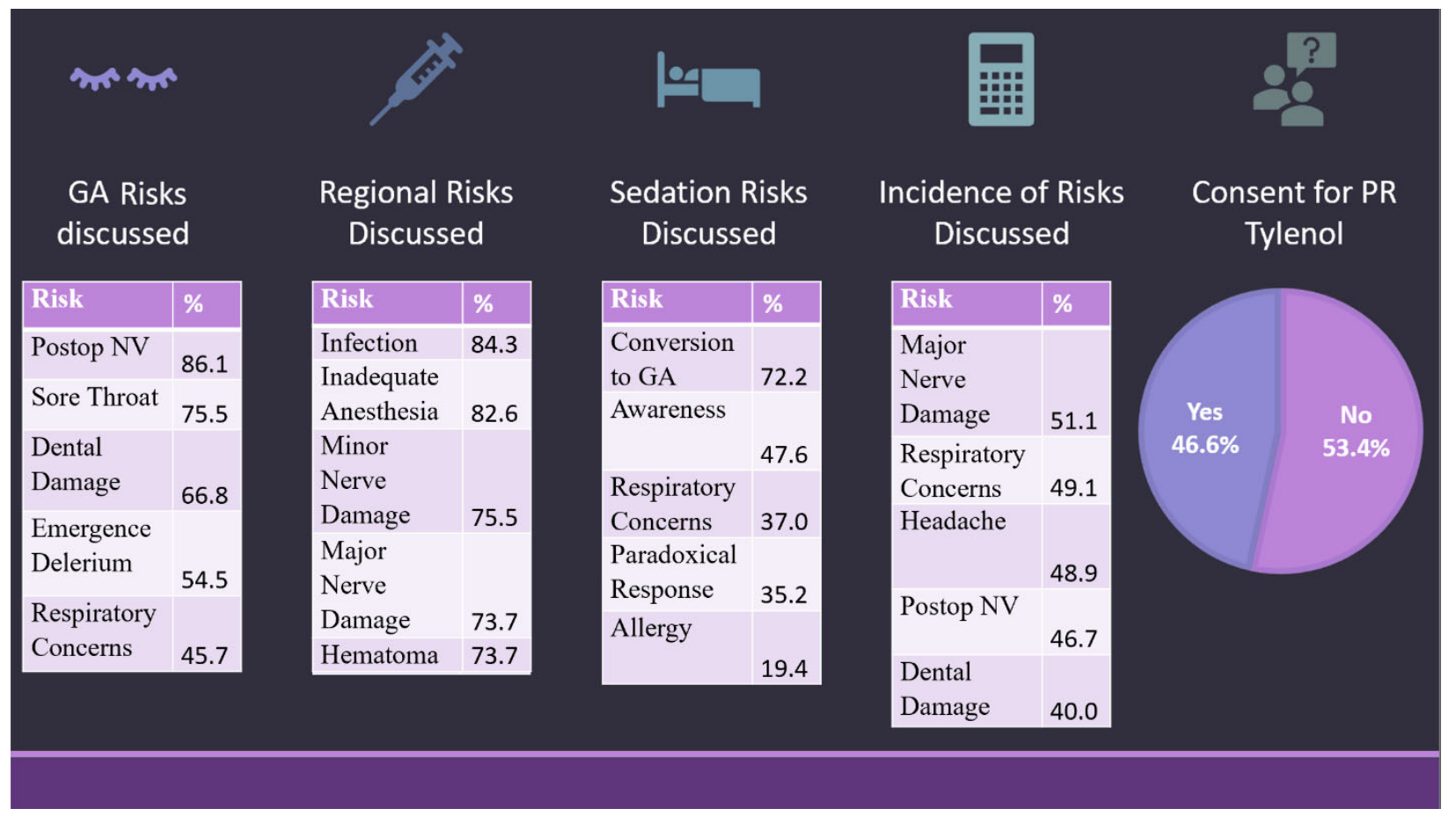




\section{Improving Outcomes in Post Anaesthetic Care Unit (PACU) for Post-Operative} Tonsillectomy Patients in a Quaternary Referral Centre: A Quality Improvement Initiative

Simon Denning ${ }^{1}$; Conor McDonnell ${ }^{1}$; Clyde Matava ${ }^{1}$; Chaminda Wijeratne ${ }^{1}$; Nikolaus Wolter ${ }^{2}$; Maisie Tsang ${ }^{1}$

1 Department of Anesthesiology and Pain Medicine, The Hospital for Sick Children, Toronto, Canada

2 Department of Otolaryngology - Head and Neck Surgery, The Hospital for Sick Children, Toronto, Canada

Introduction: Patients are presenting for tonsillectomy +/- adenoidectomy (T\&A) for treatment of obstructive sleep apnoea (OSA) [1] at an increasing rate. Our institution has a varied patient population, often with multiple co-morbidities. By comparing our current patient population and practice to local and national recommendations [2], we aim to develop a care bundle primarily to improve post-operative pain scores and, potentially, minimize peri-operative respiratory adverse events (PRAE)[2], post-operative nausea and vomiting (PONV) and emergence agitation.

Methods: Ethics approval was waived by the local REB following approval by our institutional Quality Improvement Review process. A retrospective chart review was performed using the electronic patient record. Data was extracted from cases undergoing Tonsillectomy $+/-$ Adenoidectomy between June 1, 2018 and May 31, 2019. 234 cases were identified. Excluded were cases (125) that had additional procedures; were coming from Pediatric Intensive Care Unit (PICU); or had planned PICU admissions, thereby bypassing PACU. In total 109 cases were analysed.

Results: The mean (range) age was 5.16 years (1-17) and weight was $23.73 \mathrm{~kg}$ (8-114.4) with a M:F ratio of $1.56: 1.94$ cases $(86 \%)$ were treatment for OSA, of which 48 cases $(51 \%)$ had a sleep study result with a mean maximum recorded $\mathrm{AHI}$ of $17.69(2.6-81.8) .10$ cases $(9 \%)$ were for recurrent tonsillitis and a further 5 for other reasons.

67 patients $(61 \%)$ required analgesia in PACU. 62 (57\%) complained of moderate to severe pain, 57 of whom received further opioids at a mean dose of $0.048 \mathrm{mg} / \mathrm{kg} \mathrm{ME}$.

There were 4 instances of oxygen saturations less than $90 \%$ recorded in PACU, requiring minimal intervention. Emergence agitation was recorded in 18 patients (17\%). 4 incidences $(4 \%)$ of PONV were documented, 87 cases $(80 \%)$ received ondansetron and dexamethasone intra-operatively, yet 30 patients $(28 \%)$ received dimenhydrinate in PACU.

Discussion: The population presenting for T\&A at our institution is predominantly for treatment of moderate to severe OSA. Concerns of opioid related respiratory events in PACU may explain the relatively few desaturation events contrasted with the incidence of moderate to severe pain in PACU.

Use of acetaminophen and dexamethasone (Grade A recommendation [3]), are already established perioperative practice in our centre, whilst use of NSAIDs (Grade A) is not as widely accepted by otolaryngology surgeons. Elements that show potential for low risk, high reward inclusion in a new care bundle include surgical infiltration of local anaesthetic (Grade B) and intra-operative dexmedetomidine (Grade C). 
The results of this pilot study suggest that post-operative pain can be improved through greater adherence to recent recommendations. Any future project would also monitor PONV and PRAE as balance measures.

\section{REFERENCES:}

1. Brown K. Outcome, risk, and error and the child with obstructive sleep apnea. Paediatr Anaesth. 2011 Jul;21(7):771-80. doi: 10.1111/j.1460-9592.2011.03597.x. Epub 2011 May 3.

2. Ramgolam A, Hall GL, Zhang G, Hegarty M, von Ungern-Sternberg BS. Deep or awake removal of laryngeal mask airway in children at risk of respiratory adverse events undergoing tonsillectomy-a randomized controlled trial. BJA 2018;120: 571-580.

3. Quality Based Procedure Tonsillectomy with or without Adenoidectomy Toolkit, PCMCH

\begin{tabular}{|c|l|l|l|}
\hline$\#$ & Grade & \multicolumn{1}{|c|}{ Recommendations } & The Hospital for Sick Children, Toronto \\
\hline 2.1 .2 & B & Local Anesthesia & $51(47 \%)$ had local anaesthetic applied by the surgical team \\
\hline 2.2 .1 & A & Intra-0perative Steroids & $97(89 \%)$ received intraoperative dexamethasone, mean dose $0.14 \mathrm{mg} / \mathrm{kg}$ \\
\hline 2.2 .2 & A & Acetaminophen & $99(91 \%)$ received pre-operative acetaminophen \\
\hline 2.2 .4 & A & Intra-0perative NSAIDs & $2(29)$ received pre-op ibuprofen. $47(43 \%)$ received intra-operative ketorolac \\
\hline \hline 2.2 .5 & C & $\begin{array}{l}\text { Analgesia for patients with known } 0 \\
\text { suspected 0SAS }\end{array}$ & $\left.\begin{array}{l}107(98 \%) \text { received at least one opioid intra-operatively, } 54(50 \%) \text { received two. Mean morphine equivalent (ME) dose } \\
\text { given was } 0.11 \mathrm{mg} / \mathrm{kg} . \\
75 \text { patients }(69 \%)\end{array}\right)$ were given dexmedetomidine, mean dose $0.43 \mathrm{mcg} / \mathrm{kg}$ \\
\hline
\end{tabular}

Table 1: Provincial Council for Maternal and Child Health Recommendations [3] vs our institution data 


\title{
Pediatric Dentistry Under General Anesthesia: A Saskatchewan Profile
}

\author{
Mateen Raazi ${ }^{1,2} ;$ Jennifer M. O’Brien ${ }^{1}$; Keith Da Silva ${ }^{3}$; Kristi D. Wright ${ }^{4}$
}

1 Provincial Department of Anesthesiology, University of Saskatchewan, Saskatoon, Canada

2 Provincial Department of Anesthesiology, Saskatchewan Health Authority, Saskatoon, Canada

3 College of Dentistry, University of Saskatchewan, Saskatoon, Canada

4 Department of Psychology, University of Regina, Regina, Canada

Introduction: More than 19,000 Canadian children aged 0-6 years undergo dental treatment under general anesthesia (GA) annually. National data suggest that children of low socioeconomic status, from rural areas, and from neighbourhoods with a high proportion of Indigenous peoples are more likely to receive dental care under GA. In addition to the usual risks of GA, concerns remain about the potential long-term neurodevelopmental risks of early and repeated exposure to GA. The objectives of this study were to determine the prevalence and predictive factors for dental treatment under GA in Saskatoon, Saskatchewan, a tertiary care referral center with a catchment area of 500,000 people.

Methods: Ethics approval was obtained from the local REB. We conducted a retrospective review of children aged 0-6 years who underwent dental treatment under GA in Saskatoon between the years 2015-2018. Demographic, dental diagnostic and treatment data, and the number of previous exposures to GA were collected. Quantitative analysis included comparisons of proportions and measures of associations between demographic and clinical variables.

Results: A total of 570 patient records were reviewed. The mean age of children in the sample was $4.57 \pm 0.73$ with $21.4 \%$ having required dental treatment under GA on more than 1 occasion. Fifty percent of the sample lived within a 1-hour driving distance from Saskatoon, compared to $22.7 \%$ who lived greater than 3 hours away. The dental treatment needs for the sample were complex, with children having a mean of $10.85 \pm 3.56$ teeth treated, at an average dental treatment cost of $\$ 3231.72 \pm 898.95$ and anesthesia cost of $\$ 784.33 \pm 159.81$ per child. Comparisons based on driving distance revealed that children who lived greater than 1-hour driving distance away from Saskatoon had a significantly higher severity of tooth decay, total number of teeth treated, total extractions, total crowns placed, and cost of dental treatment. Additionally, children who lived greater than 1-hour driving distance away from Saskatoon were $30 \%$ more likely to have had previous dental treatment under GA (OR: 1.3, 95\% Cl: $1.03-1.65$ ) when compared to those who lived closer.

Conclusion: Our results demonstrate that children who live greater than 1-hour away from Saskatoon have a higher burden of disease and are more likely to require repeated GA exposures for dental treatment. Further analysis is pending to uncover the incidence of dental care under GA for First Nations children. Targeted prevention strategies to reduce the burden of oral disease for high-risk children must be considered to reduce the incidence of dental cases under GA. We further recommend that Health Authorities prioritize addressing geographic disparities that exist with access to timely dental care. 
Table 1. Differences by driving distance from primary treatment site

\begin{tabular}{|l|c|c|c|}
\hline Outcome measure & Within 1-hr & Greater than 1-hr & $\mathbf{9 5 \%}$ CI \\
\hline Repeat treatment under GA (OR) & 0.80 & $1.30^{* *}$ & $0.70-0.95 ; 1.03-1.65^{*}$ \\
\hline Total time under GA in minutes (mean) & 105.16 & 103.74 & $99.9^{*} 108.97^{\mp}$ \\
\hline DMFT/dmft index |decayed, missing, filled teeth| & 10.91 & $12.14^{* * *}$ & $10.85^{*} 12.04^{\mp}$ \\
\hline Total teeth treated (mean) & 10.32 & $11.59^{* * *}$ & $10.28-11.42^{7}$ \\
\hline Total restorations placed (mean) & 8.35 & $8.90^{*}$ & $8.12^{*} 8.78^{\mp}$ \\
\hline Total extractions (mean) & 1.77 & $2.68^{* * *}$ & $1.81-2.63^{\mp}$ \\
\hline Total dental treatment cost per child (\$) & 3056.01 & $3408.99^{* * *}$ & $3025.60-3336.40^{\mp}$ \\
\hline Total anesthesia cost per child (\$) & 788.46 & 780.16 & $757.74-810.26^{\mp}$ \\
\hline
\end{tabular}

T Independent T-Test; $¥$ Chi-Squared Test; ${ }^{*} \mathrm{p}<0.05 ; * * \mathrm{p}<0.01 ; * * * \mathrm{p}<0.001$ 


\section{Population-Based Incidence and Prevalence of Child's Anxiety During Induction of Anesthesia and Efficacy of Anxiolytic Techniques From 13,523 Cases}

Julie $\mathrm{Yu}^{1}$; Monica Caldeira ${ }^{1}$; Clyde Matava ${ }^{1}$

1 The Hospital for Sick Children, Toronto, Ontario, Canada

Introduction: Preoperative anxiety in children continues to be a challenge for anesthesiologists with a reported incidence of 40 to $60 \%$ during induction of anesthesia $(1,2)$. These incidents rates have been reported from clinical trials and may not be reflected in real world practice. The goal of our study was to provide a population-based incidence of anxiety in children during induction of anesthesia, as well as identify interventions used, and their efficacy.

Methods: Ethics approval was obtained from the local REB. This was a retrospective observational study, of induction behaviour assessments of pediatric patients undergoing anesthesia at the Hospital for Sick Children. De-identified anesthesia induction behaviour assessments of patients $<18$ years of age were extracted from our Epic electronic record, between February 10th 2019 to October 31st 2019. The validated Child Induction Behavioural Assessment (CIBA) Tool, a 3-point likert scale ranking induction as smooth, moderate or difficult is used to document induction behaviour at our institution at each induction encounter $(3,4)$.

Results: 13,523 pediatric anesthesia induction behaviour assessments were extracted. 6245 $(46.2 \%)$ were mask inductions, $5662(41.9 \%)$ were intravenous inductions, $3(0.02 \%)$ were intramuscular inductions, and $1613(11.9 \%)$ did not indicate the method of induction. Using the CIBA Tool, the overall incidence of difficult induction was $7.8 \%(95 \% \mathrm{Cl}, 7.41-8.32 \%)$. The incidence of a difficult induction decreased with age as patients $<1$ year of age, 1 to 3 years of age, 4 to 12 years of age, and 13 to 17 years of age, had incidences of $10.2 \%(95 \% \mathrm{Cl}, 8.72-$ $11.9 \%), 15.0 \%(95 \% \mathrm{Cl}, 13.9-16.2 \%), 5.5 \%$ (95\% Cl, $4.9-6.1 \%)$, and $1.8 \%(95 \% \mathrm{Cl}, 1.3-2.4 \%)$, respectively. Of those that experienced mask induction, there was an overall incidence of $12.4 \%$ $(95 \% \mathrm{Cl}, 11.6-13.2 \%)$ of severe anxiety compared to an overall incidence of $3.0 \%(95 \% \mathrm{Cl}$, $2.6 \%-3.5 \%)$ in patients undergoing intravenous inductions. Parental presence was used in $41.4 \%$ of cases and reported as effective in $66.0 \%(95 \% \mathrm{Cl}, 64.8-67.2 \%)$ of patients.

Premedication was used in $22.7 \%$ of cases and reported as effective in $50.2 \%(95 \% \mathrm{Cl} 48.5$ $52.0 \%$ ) of cases.

Conclusion: This is the first study reporting a population-based incidence of anxiety during induction of anesthesia in children. The incidence is significantly lower than that reported in clinical trials. This may be a reflection of excellent screening and management of patients with severe anxiety in the perioperative setting. As expected, the incidence of anxiety is patient-age dependent. The choice of induction technique demonstrated a difference in the incidence of difficult induction. Premedication and parental presence when used, were an effective, but not always efficacious, means of managing anxiety. Future work underway is extracting similar data in a multicenter fashion, to observe differences in induction in different populations and adjusting the role of other factors. 


\section{REFERENCES:}

1. https://doi.org/10.1111/j.1460-9592.2006.01904.x

2. https://doi.org/10.1016/j.jopan.2012.01.003

3. https://doi.org/10.1016/j.jopan.2016.10.004

4. https://doi.org/10.1213/ANE.0000000000003945 


\section{PERIOPERATIVE}

\section{"Hydrate While You Wait": Application of a Pre-Procedural Oral Hydration Concept in the Ross Tilley Burn Centre}

Jordan Filion ${ }^{1,2}$, Anna Van Osch ${ }^{2,3}$, Kimberly Furtado ${ }^{3}$, Alan Rogers ${ }^{3}$, Ramona Hackett ${ }^{3}$, Gerry Hubble $^{4}$, Claude Laflamme ${ }^{5}$, Katelynn Maniatis ${ }^{2}$

1 Veterans Centre, Sunnybrook Health Sciences Centre, Toronto, Canada

2 Department of Clinical Nutrition, Sunnybrook Health Sciences Centre, Toronto, Canada

3 Ross Tilley Burn Centre, Sunnybrook Health Sciences Centre, Toronto, Canada

4 Department of Critical Care Medicine, Sunnybrook Health Sciences Centre, Toronto, Canada

5 Department of Anesthesia, Sunnybrook Health Sciences Centre, Toronto, Canada

Introduction: Although the literature strongly supports oral consumption of clear fluids up until 2 hours pre-operatively, surgical inpatients are routinely 'nil per os' (NPO) from midnight prior to their anticipated surgery and only fed again several hours after returning from the operating room ${ }^{1}$. This practice is not without significant consequences for patients with severe burn injuries, given their high nutritional requirements and the number of procedures (i.e., surgeries and dressing changes under sedation) that they undergo. Hydrate While You Wait is a preoperative hydration pathway that has been successfully implemented on other units at Sunnybrook Health Sciences Centre. This study describes how this protocol was adapted for use at the Ross Tilley Burn Centre (RTBC), with the aim of reducing fasting times and patient discomfort (subjective hunger and thirst) in the pre-procedural period.

Methods: Ethics approval was waived by the local REB. Baseline data on fasting time (including fasting time before each procedure and cumulative fasting time during admission) was collected over a 7-week period from July to September 2019. Nursing staff were instructed to ask patients to rank their subjective hunger and thirst on a scale of 1 to 10 (1 being "not at all hungry/thirsty" and 10 being "extremely hungry/thirsty") 15 minutes prior to each procedure. Analysis of procedural scheduling and extensive consultation with stakeholders informed the development of hydration cut-off times for each type of surgical case (i.e., first, second, third, and emergency cases) and procedural sedation.

Results: Data was collected on 15 patients, who underwent a combined total of 36 procedures. At baseline, patients fasted for a median of 10.5 hours on each occasion, with a median cumulative fasting time of 18.1 hours over the course of their admission. Patient survey responses $(n=18)$ revealed that baseline median subjective thirst was greater than median subjective hunger ( 7 vs 3 ), which is consistent with what has been reported in the literature ${ }^{2,3}$. Application of the proposed hydration cut-offs would have resulted in a $67 \%$ reduction in median cumulative fasting time.

Discussion/Conclusion: Adaptation and implementation of the Hydrate While You Wait initiative has strong potential to reduce fasting time, aligning practices in RTBC with best practice guidelines. Lessons learned in the implementation phase will provide insight into the feasibility of implementing a pre-procedural hydration pathway in burn units, while maintaining flexibility in procedural scheduling. 


\section{REFERENCES:}

1. American Society of Anesthesiologists Committee. (2011). Practice guidelines for preoperative fasting and the use of pharmacologic agents to reduce the risk of pulmonary aspiration: Application to healthy patients undergoing elective procedures: An updated report by the American Society of Anesthesiologists Committee on Standards and Practice Parameters. Anesthesiology, 114(3), 495-511.

2. Crenshaw, J.T., \& Winslow, E.H. (2002). Preoperative fasting: Old habits die hard. American Journal of Nursing, 102, 36-44.

3. Crenshaw, J.T., \& Winslow, E.H. (2006). Actual versus instructed fasting times and associated discomforts in women having scheduled Cesarean birth. Journal of Obstetric, Gynecologic, \& Neonatal Nursing, 35(2), 257-64. 
A Quality Improvement Initiative to Identify Peri-Operative Hyperglycaemia - The Barriers and Challenges

Vanisha Patel ${ }^{1,4}$; Sarah MacDonald ${ }^{2,4}$; Natalia Lavrencic ${ }^{3}$; Maggie Chen ${ }^{1,2}$; Natalie Clavell ${ }^{1,4}$; Warren Luksun ${ }^{2,4}$

1 Department of Anesthesiology, Toronto Western Hospital, University Health Network, Toronto

2 Department of Anesthesiology, Toronto General Hospital, University Health Network, Toronto

3 Patient Safety Office, University Health Network, Toronto

4 Department of Anesthesiology and Pain Medicine, Faculty of Medicine, University of Toronto

Introduction: The University Health Network (UHN) is an academic healthcare institution that participates in the American College of Surgeons-National Surgical Quality Improvement Program (ACS-NSQIP). Currently, the ACS-NSQIP rating for Surgical Site Infection (SSI) in the General Surgery Program at UHN has been found to "need improvement." The incidence of perioperative hyperglycemia, and its role in the development of SSI in the general surgery population at UHN is currently unknown. As a single hyperglycaemic episode (BG $>11 \mathrm{mmoL}$ ) increases risks of SSI,[1] we aimed to identify barriers to hourly glucose measurement and the incidence of $B G>10 \mathrm{mmoL}$ in the preoperative care unit (POCU) loperating and rooms in patients undergoing general surgery procedures $>2 \mathrm{hr}$ duration. The Canadian Diabetes Association recommends pre-prandial targets of $5-8 \mathrm{mmoL}$ in non-critical inpatients and $<10 \mathrm{mmoL}$ for random measurements. [2] These guidelines highlight potential methods for sampling, role of benchmarking and system performance and developing data management systems, but lack information on implementation and development of such a program. Therefore, we help address important knowledge gaps by reporting our multisite, quality improvement (QI) process.

Method: As a QI initiative, ethical review was waived. A steering committee was formed; workflow and potential measurement points were mapped to understand current and possible performance/knowledge gaps. A protocol for monitoring and managing perioperative hyperglycemia (BG $>10 \mathrm{mmoL}$ ) was developed. Fifty consecutive patients, undergoing eligible general surgery procedures were identified (Sept 2019 - Feb 2020). Hourly intraoperative glucose measurements were requested, barriers to measurement were documented by staff and follow-up focus groups/interviews conducted. Study data was collected pre, intra and postoperatively using the electronic medical record.

Results: Engagement of anesthesiologists, surgeons, endocrinologists, nurses and pharmacists allowed for interdisciplinary dialog and collaboration. Ad-hoc measurements of blood glucose in POCU and PACU revealed 6.7\% at Toronto Western Hospital (TWH) and 5.8\% at Toronto General Hospital $(\mathrm{TGH})$ measured $\mathrm{BG}>10 \mathrm{mmoL}$. A practice change was introduced, POCU and PACU nurses were asked to perform BG checks on all patients. Identified barriers included education/training surrounding glucometer use, staff workload, insulin availability and treatment pathways. Hourly intraoperative monitoring revealed $11 \%(\mathrm{TWH})$ and $38 \%(\mathrm{TGH})$ of patients had a $\mathrm{BG}>10 \mathrm{mmoL}$. Barriers in this phase included staff prompts to monitor glucose, insufficient staffing to run samples, glucometer availability and patient inaccessibility due to surgical positioning.

Discussion: This $\mathrm{Q}$ initiative highlights the complex system required to develop an infrastructure that ultimately improves perioperative glycemic measurement. Engaging relevant 
stakeholders is vital to foster leadership and drive on-going QI cycles. Education and training require continual support given the turnover of residents and nurses. Documentation, collection and monitoring of data needs to be reliable and robust, use of an electronic system can improve data quality and frequency of reporting.[3] Other institutions wishing to improve perioperative glycemic control may benefit from our experience and QI roadmap. Future work will address the barriers identified to ensure glucose can be measured readily prior to implementing therapies.

\section{REFERENCES:}

1. Diabetes Canada Clinical Practice Guidelines Expert Committee. Diabetes Canada 2018 Clinical Practice Guidelines for the Prevention and Management of Diabetes in Canada. Can J Diabetes 2018;42:S1-325.

2. Dronge AS, Perkal MF, Kancir S, et al. Long-term glycemic control and postoperative infectious complications. Arch Surg 2006;141:375-80; discussion 380.

3. Khan JS, Jibb LA, Busse JW, et al. Electronic Versus Traditional Data Collection: A Multicenter Randomized Controlled Perioperative Pain Trial. Canadian Journal of Pain 2019;3:16-25. 
A Survey of the Anesthesia Assistant Profession in Canadian Teaching Hospitals: 12 Years Later

Claire Ward ${ }^{1}$; Allana Munro ${ }^{1,2,3}$; Chantal Houser ${ }^{1}$; Paul Brousseau ${ }^{3}$

1 Department of Women's \& Obstetric Anesthesia, IWK Health Centre, Halifax, Canada

2 Department of Anesthesia, Pain Management and Perioperative Medicine, Dalhousie University, Halifax, Canada

3 Department of Anesthesia, Pain Management and Perioperative Medicine, QEII Health Sciences Center, Halifax, Canada

Introduction: The role of the Anesthesia Assistant (AA) varies widely across Canada, both in level of education and clinical responsibility. As of January 2019, respiratory therapists must pass a national certification exam to achieve the "Certified Clinical Anesthesia Assistant" (CCAA) designation. The intent is to regulate AA education programs and standardize the skillset of AA graduates. A survey completed in $2007,{ }^{1}$ examined the role of AAs at academic hospitals across Canada, focusing on the most common technical and clinical responsibilities of the profession.

Objectives: This study aims to identify the educational background and clinical roles of AAs in Canadian University Departments of Anesthesia (ACUDA hospitals) and determine how they have changed over the last 12 years.

Methods: Ethics approval was obtained from the local REB. A non-interventional survey of ACUDA hospitals was completed between October 21st, 2019 and January 30th, 2020. Hospital managers were contacted and asked to complete a standardized, telephone-based survey. Data regarding AA education, clinical tasks, and scope of practice was collected, analyzed and compared to study findings of the same ACUDA hospitals from 2007. Analysis was completed using descriptive statistics.

Results: A total of 15/17 ACUDA hospitals were available for survey participation. On average, there were 33 operating rooms (ORs) per hospital, with 100\% having some level of AA involvement. Only 5 hospitals (33\%) had AAs with formal certification. There were approximately 22 AAs per hospital. AAs spent $25 \%$ of their time working in areas outside of the main ORs, and $47 \%$ of sites (7/15) had 24 -hour, on-site AA coverage. The most common technical responsibilities of AAs included bronchoscopy assisting/cleaning (14/15), epidural/spinal anesthesia assists (14/15), and troubleshooting equipment (13/15). The most common clinical duties included monitoring for general anesthesia and OR anesthesiologist relief $(15 / 15 ; 14 / 15)$, intravenous catheter insertion (15/15), and medication delivery (15/15). Compared to 2007, the largest increase in AA responsibilities was observed for monitoring general anesthetics (43.8\%), OR anesthesiologist relief (43.3\%) and administration of vasopressors (43.8\%). Additionally, increases were observed for administration of induction agents $(37.5 \%)$ and maintenance $(37.5 \%)$, recovery room care $(33.4 \%)$ and central line insertion (20\%). A comparison of 2007 to 2019 responsibilities is represented in figure 1.

Conclusion: There remains considerable variability in educational background and certification of AAs across Canadian academic anesthesia departments. The majority (67\%) of departments have assistants on staff that have not completed a formal AA certification program. Currently, AAs spend a quarter of their time out of OR; however, their most common responsibility is 
monitoring for general anesthesia. Compared to 2007, OR responsibilities have increased significantly, demonstrating a shift towards more clinically-based skills.

\section{REFERENCES:}

1. Brousseau, P., Nickerson, J., \& Murphy, M. (2007). A survey of anesthesia assistant utilization in Canadian teaching hospitals. Canadian Journal of Anesthesia/Journal canadien d'anesthésie, 54, 44264-44264

Figure 1. Type and percentage of AA clinical duties performed in Canadian teaching hospitals in 2007 and 2019

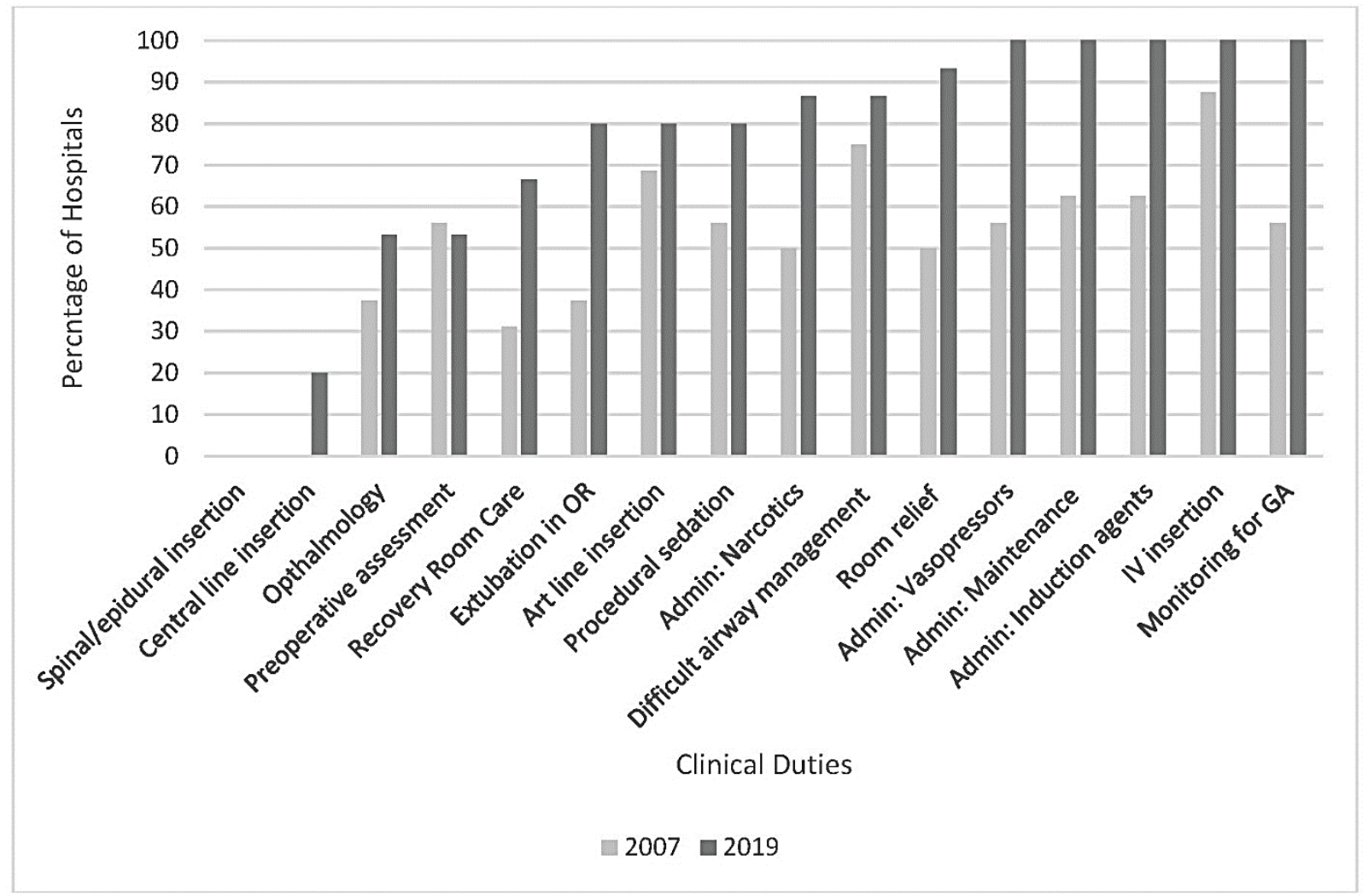




\section{Anesthesia for Total Joint Arthroplasty in a Smaller Community Hospital - An}

\section{Observational Quality Improvement Study}

\section{David Ohrling ${ }^{1,2}$; Alghin Wilson ${ }^{2}$}

1 Department of Anesthesia, Collingwood General and Marine Hospital, Collingwood, Canada 2 Rural Ontario Medical Program, Collingwood, Canada

Introduction: As part of a larger Quality Improvement project aiming to reduce LOS for Total Hip Arthroplasty (THA) and Total Knee Arthroplasty (TKA) patients, this retrospective observational study aimed to examine anesthesia related factors that might influence patients' perioperative experience and be considered for future intervention.

Methods: Ethics approval was received from the local REB. Charts were reviewed for 143 consecutive patients who underwent single Total Hip or Knee Arthroplasty at a smaller community hospital (Collingwood General \& Marine - 78 total beds, 3 Orthopedic Surgeons, 6 Family Practice Anesthetists). Type of anesthetic given (Spinal vs GA) and resulting pain scores and narcotic usage, use of intrathecal epimorphine and its correlation to catheter rates, nausea rates and pain scores/narcotic use in the first 24 hours, and the effect of femoral nerve blocks on pain scores and narcotic use in the first 24 hours for TKA patients were analyzed.

Results: Overall, $92 \%$ of THA and $90 \%$ of TKA patients received a spinal anesthetic. Patients who had spinal anesthesia had significantly lower maximum pain scores, total narcotic use in the first 24 hours and a significantly longer time to first narcotic use than patients who had general anesthetic. For patients receiving spinal anesthesia, the catheter rate postoperatively for inability to void was $22 \%$ with a significant correlation with epimorphine dose (R squared $0.956, p=0.004)$. Nausea rate overall was $29.2 \%$ which was not significantly associated with the use of PONV prophylaxis or epimorphine dose. Trends were observed for both THA and TKA patients between epimorphine dose and maximum pain scores, time to first narcotic and total narcotic requirements in the first 24 hours postop however these were not significant. The use of femoral nerve blocks for TKA patients significantly reduced narcotic requirements in the first 24 hours postop with non-significant trends towards lower maximum pain scores and longer times to first narcotic dose.

Conclusions: Our data suggests that for THA and TKA patients in our institution, spinal anesthesia is preferred, epimorphine use should not be routine and for TKA patients, femoral nerve blocks should be encouraged. During the study period, $75 \%$ of THA and $72 \%$ of TKA patients were discharged on postop day 1 which will serve as a baseline for future comparison. 


\title{
Case Report: Using Dexmedetomidine Infusion for Sedation in Spontaneously Breathing Patient with Myotonic Dystrophy
}

\author{
April (YiChen) Liu ${ }^{1}$, Andrea Dower ${ }^{2}$, Saeda Nair ${ }^{2}$
}

1 Undergraduate Medicine, Michael G. DeGroote School of Medicine, Hamilton, Canada 2 Department of Anesthesiology, Hamilton Health Sciences, Hamilton, Canada

Introduction: Myotonic dystrophy type I (DM) is the most common muscular dystrophy in adults. It affects numerous body systems including the musculoskeletal, respiratory, cardiovascular, gastrointestinal, and central nervous systems which complicates their anesthetic management. Patients are prone to myotonia, a prolonged muscle contraction, which is triggered by succinylcholine, neostigmine, hypothermia, shivering, and surgical stimulation. Dystrophic organization of skeletal muscles result in weakness, often involving the facial, laryngeal, and respiratory muscles. Thus, patients have increased risks of post-operative complications including respiratory depression and pneumonia. DM patients also have low central ventilatory drives, increasing their sensitivity to respiratory depressants, including opioids. They are prone to arrhythmias and both systolic and diastolic dysfunction because of fibrosis of cardiac myocytes. Volatile agents may exacerbate these preexisting cardiac comorbidities. Smooth muscle involvement slows peristalsis in the gastrointestinal tract, increasing the risk of aspiration and prolonged post-operative ileus time.

Case Presentation: Patient consent was obtained for publication of this case. A 42 year old male with DM diagnosed in early adulthood presented for septoplasty and bilateral inferior turbinate reductions due to a deviated septum. His disease involvement included: 1) severe upper and lower extremity myotonia and weakness; 2) pulmonary impairment with nonobstructive patterns; 3) first-degree atrioventricular block and reduced ejection fraction. He uses bilevel positive airway pressure, a cough assist device, and is paced $3 \%$ of the time with a single chamber pacemaker.

To reduce potential complications associated with opioid use and a general anesthetic, an opioid-free anesthetic was planned using local infiltration and dexmedetomidine infusion for sedation. $0.25 \%$ bupivacaine with epinephrine was injected into septum, bilateral inferior turbinates, nasal mucosa, and posterior septum. Twenty-three micrograms of dexmedetomidine was given as bolus doses over the first 6 minutes. Then, an infusion was started at $0.4 \mathrm{mcg} / \mathrm{kg} / \mathrm{hr}$ for 20 minutes, increased to $0.5 \mathrm{mcg} / \mathrm{kg} / \mathrm{hr}$ for the next five minutes, and again to $0.7 \mathrm{mcg} / \mathrm{kg} / \mathrm{hr}$ for ten minutes. It was decreased to $0.5 \mathrm{mcg} / \mathrm{kg} / \mathrm{hr}$ for the remaining 25 minutes of the case. Oxygen was applied via facemask at eight liters per minute and the patient was kept spontaneously breathing. The patient remained hemodynamically stable and was kept overnight for monitoring. The post-operative course was uneventful.

Conclusion: Dexmedetomidine is a highly selective $\alpha 2$-adrenergic receptor agonist that has the ability to provide sedation, analgesia, and anxiolysis with a stable hemodynamic profile. Avoiding both opioids and a general anesthetic in patients with DM decreases their risk of respiratory and cardiovascular complications. Communication with the surgical team was imperative to the success of using sedation for this procedure. This is the first case report of using dexmedetomidine infusion for sedation in a spontaneously breathing patient with DM and 
advocates for its efficacy and safety in these patients with multiple comorbidities.

\section{REFERENCES:}

1. Bird TD. Myotonic Dystrophy Type 1. Adam MP, Ardinger HH, Pagon RA, et al., editors. GeneReviews. Seattle, WA: University of Washington: 1993-2020.

2. Turner C, Hilton-Jones D. Myotonic dystrophy: diagnosis, management and new therapies. Current Opinion in Neurology. 2014; 27(5):599-606. doi: 10.1097/WCO.0000000000000128

3. Mathieu J, Prevost C. Epidemiological surveillance of myotonic dystrophy type 1: a 25-year population-based study. Neuromuscul Disord. 2012; 22: 974-979. doi: 10.1016/j.nmd.2012.05.017

4. Veyckemans D, Scholtes JL. Myotonic Dystrophies type 1 and 2: anesthetic care. Pediatric Anesthesia. 2013; 23 (9), 794-803. doi:10.1111/pan.12120

5. Mangla C, Bais K, Yarmush J. Myotonic Dystrophy and Anesthetic Challenges: A Case Report and Review. Case Rep Anesthesiol. 2019; 2019: 1-9. doi: 10.1155/2019/4282305

6. Go R, Wang D, Ludwin D. Anesthetic Considerations in a Patient with Myotonic Dystrophy for Hip Labral Repair. Case Rep Anesthesiol. 2017; 2017: 6408956. doi: 10.1155/2017/6408956 7. Ferschl M, Moxley R, Day J, Gropper M. Practical Suggestions for The Anesthetic Management of A Myotonic Dystrophy Patient. Myotonic. 2019. https://www.myotonic.org/sites/default/files/pages/files/Myotonic-Anesthesia-DM1-2019-1105.pdf

8. Kirzinger L, Schmidt A, Kornblum C, Schneider-Gold C, Kress W, Schoser B. Side effects of anesthesia in DM2 as compared to DM1: a comparative retrospective study. European Journal of Neurology. 2010;17(6):842-845. doi: 10.1111/j.1468-1331.2009.02942.x.

9. Mathieu J, Allard P, Gobeil G, et al. Anesthetic and surgical complications in 219 cases of myotonic dystrophy. Neurology. 1997; 49: 1646-1650. doi:

https://doi.org/10.1212/WNL.49.6.1646

10. Mathieu J, Allard P, Potvin L, Prévost C, Begin P. A 10-year study of mortality in a cohort of patients with myotonic dystrophy. Neurology. 1999;52(8):1658-1662. doi:

10.1212/wnl.52.8.1658.

11. Bianchi ML, Losurdo A, Di Blasi C, et al. Prevalence and clinical correlates of sleep disordered breathing in myotonic dystrophy types 1 and 2. Sleep \& Breathing. 2014;18(3):579589. doi: 10.1007/s11325-013-0921-5.

12. Gaszynski T. Opioid-free general anesthesia in patient with Steinert syndrome (myotonic dystrophy): case report. Medicine. 2016; 95(37):4885. doi: 10.1097/MD.0000000000004885 13. Kaur M, Singh PM. Current role of dexmedetomidine in clinical anesthesia and intensive care. Anesth Essays Res. 2011; 5(2): 128-133. doi: 10.4103/0259-1162.94750.

14. McNally E, Sparano D. Mechanisms and management of the heart in Myotonic Dystrophy. Heart. 2011 Jul; 97(13): 1094-1100. doi: 10.1136/hrt.2010.214197 


\section{Dynamic LVOT Obstruction with Severe Hemodynamic Instability after Spinal Anesthesia}

Zamper RPC ${ }^{1}$; Fayad $\mathrm{A}^{2}$; Jones $\mathrm{P}^{3}$

1 Department of Anesthesiology and Perioperative Medicine, University Hospital - London Health Sciences Centre, Western University, London, Ontario, Canada.

2 Department of Anesthesiology and Perioperative Medicine, University Hospital - London Health Sciences Centre, Western University, London, Ontario, Canada.

3 Department of Anesthesiology and Perioperative Medicine, University Hospital - London Health Sciences Centre, Western University, London, Ontario, Canada.

Introduction: Perioperative dynamic left ventricular outflow tract (LVOT) obstruction is not uncommon and its diagnosis might be a challenge. Perioperative dynamic LVOT obstruction may occur due to surge of catecholamine, hyperdynamic status or hypovolemia in patients with concentric left ventricular hypertrophy. Although rare, patients with sigmoid septum may exhibit dynamic LVOT obstruction. We present a case of dynamic LVOT obstruction in a patient with sigmoid septum, small and hypertrophic left ventricle (LV).

Case Report: A 76-yr-old female patient with isolated systolic hypertension (190-200 mmHg) and hypothyroidism presented for a left total knee arthroplasty. Preoperatively she denied any cardiac symptoms, and ECG was showing normal sinus rhythm. The transthoracic echocardiogram (TTE) revealed a concentric LV hypertrophy with small cavity and proximal septal thickening. Normal systolic function with myocardial perfusion imaging (MIBI) negative for myocardial ischemia.

At admission, her vitals showed a blood pressure (BP) of $230 / 80 \mathrm{mmHg}$ and heart rate (HR) of $80 \mathrm{bpm}$. She was fasting for a period of 16 hours. After receiving a spinal anesthesia in the block room, she became hemodynamically unstable with systolic BP of $50 \mathrm{mmHg}$, treated with boluses of phenylephrine and fluid resuscitation with full recovery.

In the operating room, before the procedure starts, she became bradycardic (HR $30 \mathrm{bpm})$, complaining of lightheadness and nausea, treated with 0,2 mg of glycopirrolate, which increased her HR to $135 \mathrm{bpm}$. She remained hemodynamically stable but complaining of chest pain. After discussing the findings with the surgical team and a senior anesthesiologist, cardiology was consulted, and the decision was made to carry on with the surgery as planned. The patient was becoming asymptomatic as the HR decreased to $110 \mathrm{bpm}$. She remained asymptomatic throughout, an intraoperative point-of-care ultrasound cardiac exam was performed and showed a hyperdynamic LV with signs of dynamic LVOT obstruction as shown by Doppler late systolic peak velocity (Figure 1). Preoperative echocardiogram images were further reviewed and showed a normal LVOT Doppler pattern.

The patient remained asymptomatic post-operatively, with a normal ECG and no increase in troponins, being discharged from the hospital on POD3.

Discussion: It is currently assumed that LVOT obstruction is a dynamic phenomenon, the occurrence of which requires the coexistence of two elements: 1) predisposing anatomic factors and 2) a physiological condition that induces such a phenomenon. In our case, anatomic factors are a small hypertrophic LV cavity with a sigmoid septum, and the physiological conditions are 
related to a catecholamine driven anxious patient, volume depleted due to prolonged fasting period and with decreased afterload due to the spinal anesthesia. Treatment is based on reducing heart contractility and rate (sedation and beta blockers), increasing the pre-load (with fluid resuscitation) and afterload (with vasopressors).

\section{REFERENCES:}

1. Sobczyc D. Dynamic left ventricular outflow tract obstruction: underestimated cause of hypotension and hemodynamic instability. J Ultrason 2014 Dec;14(59):421-7.

2. Fayad A. Left Ventricular Outflow obstruction in a patient with undiagnosed hypertrophic obstructive cardiomyopathy. Can J Anaesth 2007 Dec;54(12):1019-20.

3. Jain P, Patel PA, Fabbro M. Hypertrophic Cardiomyopathy and Left Ventricular Outflow Tract Obstruction: Expecting the Unexpected. J. Cardiothorac. Vasc. Anesth. 2018 Feb;32(1):467-477.

4. Hioki $\mathrm{H}$ et al. Dynamic left ventricular outflow tract obstruction due to anemia in a 71-year old patient with sigmoid septum. J Cardiol Cases 2010 Jan 12;1(3)e144-e146.

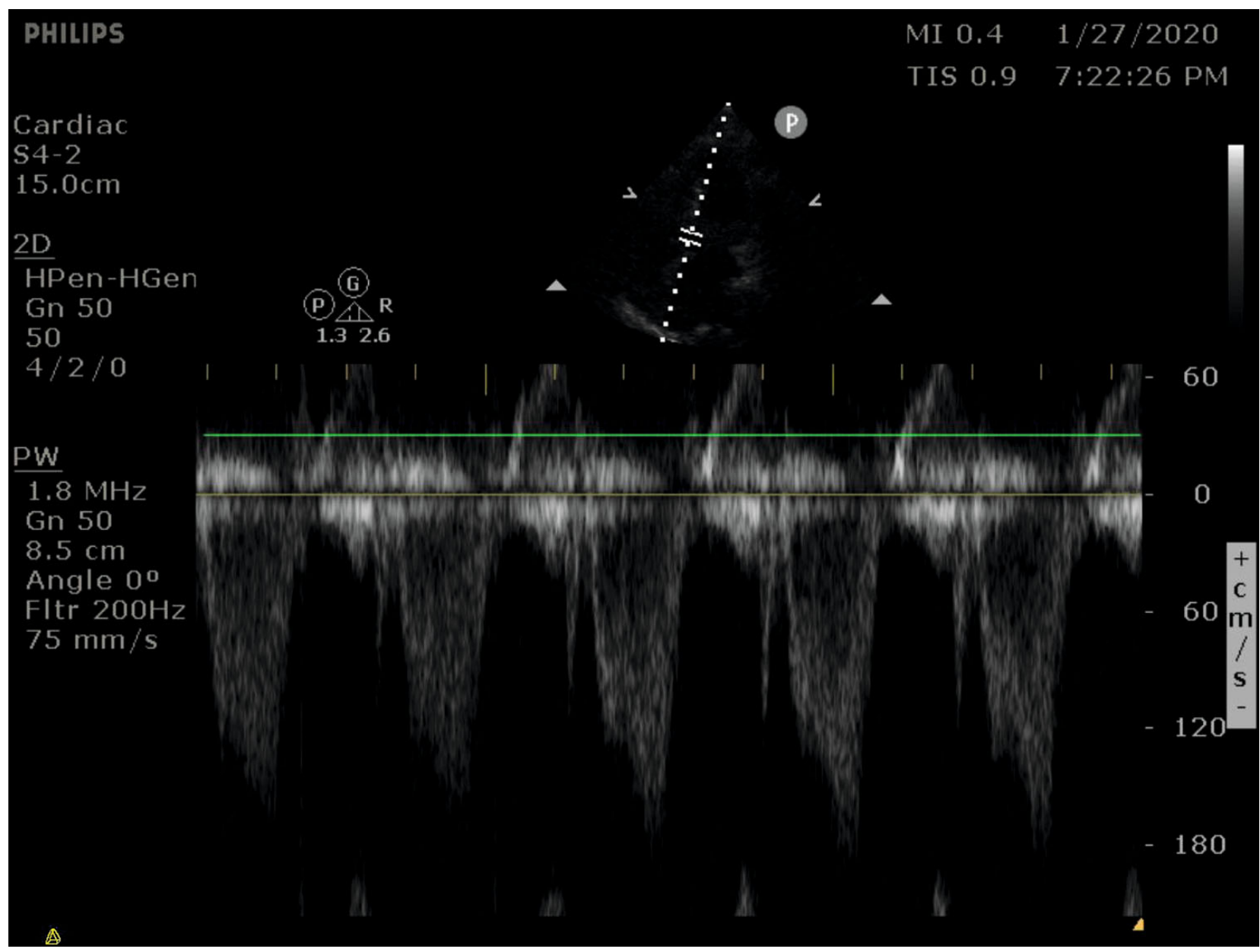

Figure 1 - Intraoperative point-of-care ultrasound cardiac exam 


\section{Effectiveness of Preoperative Patient Blood Management Strategies in Orthopedic Reconstructive Surgery}

John Peel ${ }^{1}$; Thomas Prasloski ${ }^{2}$; Garrett Barry ${ }^{3}$; Jack Huang ${ }^{2}$; Lawrence Sham ${ }^{4}$; Bas Masri ${ }^{5}$; Jacqueline D Trudeau ${ }^{2,4,6}$

1 Department of Anesthesia, University of Toronto

2 Department of Anesthesiology, Pharmacology and Therapeutics, Faculty of Medicine, University of British Columbia

3 Department of Anesthesia, Pain Management and Perioperative Medicine, Dalhousie University

4 Transfusion Medicine Service, Vancouver General Hospital

5 Department of Orthopaedics, Vancouver General Hospital

6 Department of Anesthesiology and Perioperative Care, Vancouver General Hospital

Introduction: Both preoperative anemia and red blood cell transfusion are associated with increased risk of morbidity, prolonged hospitalization, and mortality1. Preoperative treatment of anemia may reduce transfusion in patients undergoing primary hip and knee reconstructive surgery - procedures with historical transfusion rates as high as $68 \%$. We sought to characterize the relationship between preoperative $\mathrm{Hgb}$ and transfusion among patients undergoing primary hip and knee replacement at our centre2-4. Further, we compared the efficacy of interventions used at our centre to treat anemia preoperatively.

Methods: Ethics approval was obtained from the local REB. We conducted a retrospective cohort study of patients undergoing primary hip or knee replacement between January 2010 and May 2019. Patient-level data was collected from existing databases of orthopedic procedures performed at our centre, our Perioperative Blood Management Program (PBMP) clinic, and from our transfusion records. Patients in the PBMP were prescribed iron and/or erythropoietin at their anesthesiologist's discretion. We excluded patients undergoing revision or bilateral arthroplasty and patients with documented refusal of blood products, known bleeding diathesis, or hemoglobinopathy. Logistic regression modelled the relationship between preoperative $\mathrm{Hgb}$ and the probability of transfusion. Linear regression modelled the effect of iron and erythropoietin. All analyses were performed at the 95\% level using $R$ ( $R$ version 3.6.1).

Results: During the study period 13113 patients underwent hip or knee arthroplasty, of which $2532(19.3 \%)$ were anemic preoperatively and $288(2.2 \%)$ received transfusion. The odds of transfusion are 1.9 [1.4-2.5] times higher in hip arthroplasty compared to knee replacement, adjusting for preoperative $\mathrm{Hgb}(\mathrm{p}<0.001)$. The adjusted odds of transfusion are 2.5 [2.3-2.7] times higher for each $10 \mathrm{~g} / \mathrm{L}$ decrease in preoperative $\mathrm{Hgb}$, and 8.8 [6.7-11.8] times higher for anemic patients compared to non-anemic patients $(p<0.001)$ (Figure 1a). Adjusting for procedure and compared to no preoperative treatment of anemia, oral iron increased $\mathrm{Hgb} 5.1$ [1.9-8.2] $\mathrm{g} / \mathrm{L}(\mathrm{p}=0.0015)$ and decreased the odds of transfusion (OR 0.28 [0.16-0.51], $p<0.0001)$; IV iron increased Hgb 8.5 [5.0-12.0] $\mathrm{g} / \mathrm{L}(\mathrm{p}<0.0001)$ and decreased the odds of transfusion (OR 0.14 [0.07-0.28], $p<0.0001)$; erythropoietin increased Hgb 8.5 [4.5-12.5] $\mathrm{g} / \mathrm{L}(\mathrm{p}<0.0001)$ but had no significant impact on transfusion (OR 0.68 [0.33-1.41], $p=0.303$ ); combination therapy increased $\mathrm{Hgb} 9.9$ [6.0-13.8] $\mathrm{g} / \mathrm{L}(\mathrm{p}<0.0001)$ and decreased the odds of transfusion (OR 0.41 [0.20-0.83], $p=0.013$ ) (Figure 1b). 
Conclusions: Preoperative Hgb and anemia are strongly associated with transfusion in primary hip and knee arthroplasty. Iron and erythropoietin significantly increase Hgb preoperatively whereas only iron was found to decrease transfusion. These findings suggest that iron supplementation, with or without erythropoietin, before reconstructive orthopedic surgery can decrease transfusion and may therefore improve outcomes.

\section{REFERENCES:}

1. Jans $\varnothing$, Jørgensen $C$, Kehlet $H$, Johansson PI, Lundbeck Foundation Centre for Fast-track Hip and Knee Replacement Collaborative Group. Role of preoperative anemia for risk of transfusion and postoperative morbidity in fast-track hip and knee arthroplasty. Transfusion. 2013;54(3):717-726. doi:10.1111/trf.12332.

2. Ferraris VA, Davenport DL, Saha SP, Austin PC, Zwischenberger JB. Surgical Outcomes and Transfusion of Minimal Amounts of Blood in the Operating Room. Arch Surg. 2012;147(1):49-55. doi:10.1001/archsurg.2011.790.

3. Gonzalez-Porras JR, Colado E, Conde MP, Lopez T, Nieto MJ, Corral M. An individualized pre-operative blood saving protocol can increase pre-operative haemoglobin levels and reduce the need for transfusion in elective total hip or knee arthroplasty. Transfusion Medicine. 2009;19(1):35-42. doi:10.1111/j.1365-3148.2009.00908.x.

4. Hart A, Khalil JA, Carli A, Huk O, Zukor D, Antoniou J. Blood Transfusion in Primary Total Hip and Knee Arthroplasty. Incidence, Risk Factors, and Thirty-Day Complication Rates. The Journal of Bone and Joint Surgery-American Volume. 2014;96(23):1945-1951.

doi:10.2106/JBJS.N.00077.
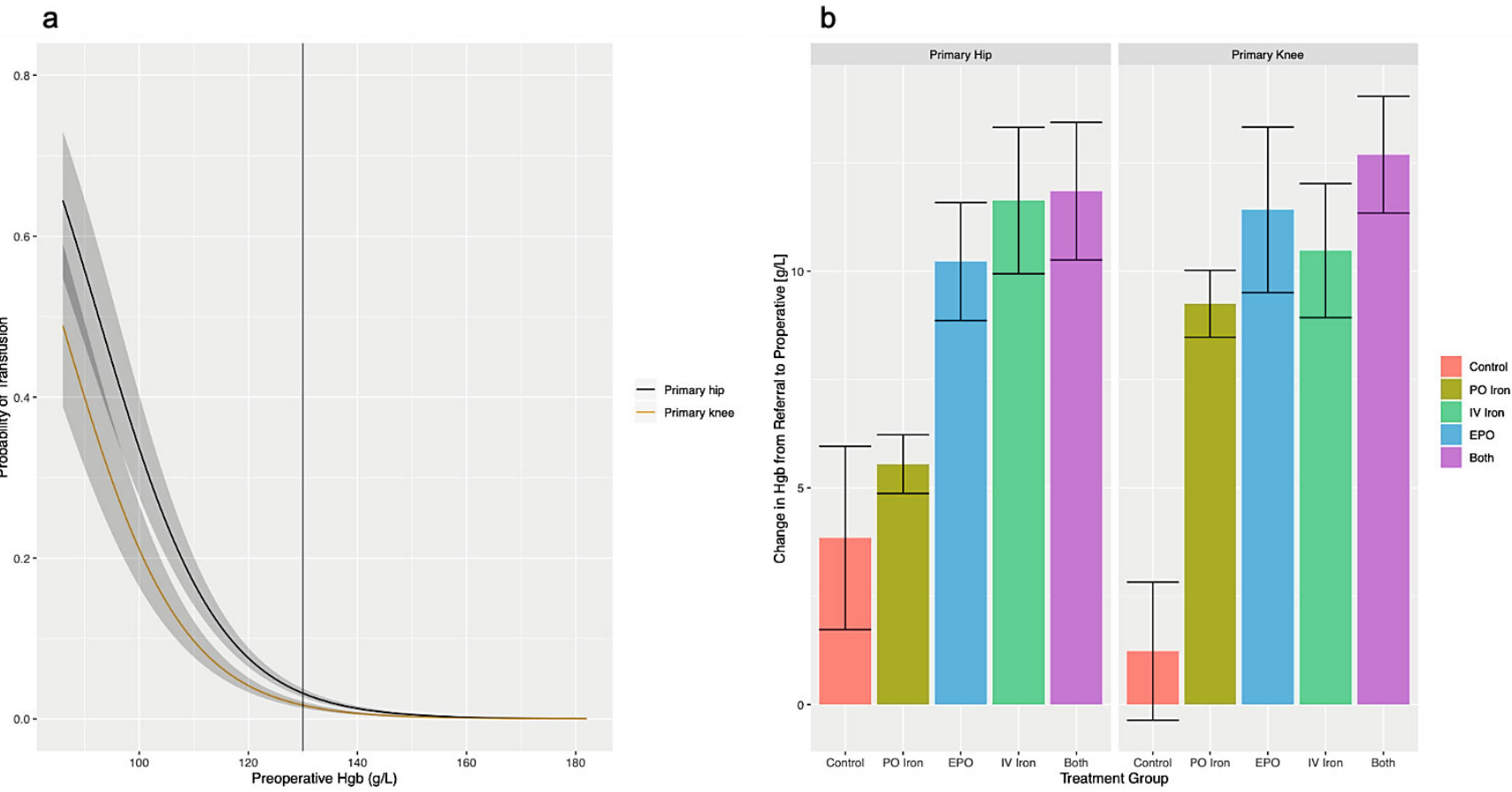
Health Resource Utilization Associated with The Implementation of The Canadian Cardiovascular Society Perioperative Guidelines for Patients Undergoing Non-Cardiac Surgery at an Academic Hospital

Simon Feng ${ }^{1}$; Dan Mclsaac ${ }^{1,2,3}$; Josh Montroy ${ }^{3}$; Sylvain Gagne ${ }^{1}$; Cedric Godbout-Simard ${ }^{1}$; Michelle Moody ${ }^{1}$; Madison Foster ${ }^{3}$; Chris Johnson ${ }^{4}$; James Chan ${ }^{4}$; Samantha Halman ${ }^{4}$; Jaclyn Ernst ${ }^{4}$; Stuart Oake ${ }^{5}$ Manoj Lalu ${ }^{1,3}$

1 University of Ottawa, Department of Anesthesiology and Pain Medicine, Ottawa, Canada 2 ICES, Toronto, Canada

3 Ottawa Hospital Research Institute, Ottawa, Canada

4 University of Ottawa, Department of Medicine, Ottawa, Canada

5 Department of Surgery, University of Ottawa and The Ottawa Hospital

Background: The Canadian Cardiovascular Society (CCS) guidelines for patients undergoing non-cardiac surgery address the lack of standardized approaches to the management of patients at risk of perioperative cardiovascular complications through preoperative brain natriuretic peptide testing or troponin ( $\mathrm{Tnl}$ ) testing with electrocardiograms. ${ }^{1}$ The implementation of these guidelines has not been systematically evaluated, and we lack data on adherence and impacts on healthcare resource utilization. We recently employed a multidisciplinary teambased approach to implement CCS guidelines at an academic hospital, including a quasiexperimental evaluation of changes in adherence, costs, length of stay, and complications.

Methods: Ethics approval was obtained from the local REB. We used an interrupted time series (ITS) design to study the effects of the CCS guidelines (August 2018 - February 2019), using routinely collected data. We included elective, non-cardiac surgery patients with: i) expected length of stay $\geq 1$ day, and ii) age $\geq 65$ or age $45-64$ with a revised cardiac risk index of $\geq 1$ or severe cardiopulmonary disease (peripheral artery disease, pulmonary hypertension, and obstructive cardiac pathology). Our primary outcome was guideline adherence (postoperative Tnl monitoring). Our secondary outcome was appropriate follow-up care (consult for Tnl positive patients). Tertiary outcomes included additional testing (electrocardiograms, perfusion scans, etc.), costs, length of stay and complications. The effect of guideline implementation was estimated using an ITS analysis based on Box-Jenkins methods for autoregressive integrated moving average models; we estimated the association of implementation with changes in the log-transformed rate of outcomes in each time period, adjusting for the underlying trend and post-implementation trend.

Results: We included 1,421 patients over the course of the study (706 pre-implementation and 715 post-implementation). Baseline demographic variables were balanced pre versus post. Table 1 provides outcomes rates pre vs post. Adherence to Tnl testing following the implementation increased 21.79 -fold $(95 \% \mathrm{Cl} 10.59-44.79)$, without an increase in length of stay (RoM 1.05; 95\% Cl 0.69-1.60) or hospitalization costs (RoM 1.10; 95\% Cl 0.76-1.60). We also found no strong evidence of change in complications rates (RoM 0.99; 95\% Cl 0.62-1.59). In patients who had elevated Tnl following guideline implementation ( $n=64,8.9 \%)$, the majority (84.4\%) received appropriate follow-up care in the form of a general medicine or cardiology consult, all (100\%) received at least one electrocardiogram, and half $(51.6 \%)$ received st Insct 
one coronary diagnostic test (CT angiogram, cardiac perfusion scan, or a percutaneous intervention).

Conclusions: Systematic, multidisciplinary implementation of CCS postoperative monitoring guidelines led to a substantial increase in postoperative troponin screening in at risk-patients, including a high rate of appropriate follow up in screen-positive patients. These improvements in guideline-based care were not accompanied by increases in healthcare resource use. Largescale multicenter evaluations of CCS guideline implementation are needed to evaluate the impact of guidelines on patient outcomes.

\section{REFERENCES:}

1. Duceppe E, Parlow J, MacDonald P, et al. Canadian Cardiovascular Society Guidelines on Perioperative Cardiac Risk Assessment and Management for Patients Who Undergo Noncardiac Surgery. Can J Cardiol. 2017;33(1):17-32. doi:10.1016/j.cjca.2016.09.008

$\begin{array}{lcc}\text { Period } & \text { Pre-implementation } & \text { Post-implementation } \\ \text { No. of patients }(n) & 706 & 715 \\ \text { Tnl Testing Adherence }(n, \%) & 40(5.7 \%) & 496(69.4 \%) \\ \text { Elevated Troponin ( } \mathrm{n}, \%) & 19(2.7 \%) & 64(9.0 \%) \\ \text { Length of stay (mean, sd) } & 4.3(6.7) & 4.0(5.9) \\ \text { In-hospital cost (mean, sd) } & \$ 12,833.32 & \$ 11,317.01(\$ 7,313.29) \\ & (\$ 15,378.31) & 78(10.9 \%) \\ \text { Any complication ( } \mathrm{n}, \%) & 81(11.5 \%) & 54(84.4 \%) \\ \text { Received Appropriate Follow-up Care (of } & & 19(29.7 \%) \\ \text { elevated Tnl patients) } & & 64(100 \%) \\ \text { Received at least one echo (of elevated Tnl } & & \\ \text { patients only) } & & 3.84(2.77) \\ \text { Received at least one ECG (of elevated Tnl } & 33(51.6 \%) \\ \text { patients only) } & \\ \text { ECGs per patient (mean, SD) } & \\ \text { At least one cardiac test (CT angio, perfusion } & \\ \text { scan, perc. Interventions) } & & \\ \text { Table } 1 \text { displays the difference in outcomes rates pre- and post-implementation of the CCS guidelines. }\end{array}$




\section{In-Home, Computerized Assessment for Investigating Perioperative Neurocognitive} Deficit in Elderly Surgical Populations

Michael Schmidt'1,2; Anne Johnson ${ }^{3}$; Gail Eskes $3,4,5$

1 Department of Anesthesia, Pain Management \& Perioperative Medicine, Dalhousie University, Halifax, NS, Canada

2 Medical Staff, QEII Health Sciences Centre, Nova Scotia Health Authority, Halifax, NS, Canada

3 Cognitive Health and Recovery Research Laboratory, Department of Psychiatry, Dalhousie University, Halifax, NS, Canada

4 Department of Psychology and Neuroscience, Dalhousie University, Halifax, NS, Canada

5 Department of Psychiatry, Dalhousie University, Nova Scotia, Halifax, NS, Canada

Introduction: 200 million people worldwide receive general anesthesia (GA) each year. ${ }^{1}$ Unfortunately, significant post-operative changes in patient cognition may occur after surgery with the greatest deficits in elderly patients ${ }^{2}$. Despite the potential long-lasting complications of perioperative neurocognitive deficits (PND) ${ }^{3}$, there is currently no consistent and easy method to identify a patient's risk of developing PND and monitor its long-term outcomes.

The Dalhousie Computerized Attention Battery (DalCAB) is a patient-friendly tool available online that assesses fundamental aspects of cognition that could bridge this gap ${ }^{4}$. A 2016 pilot study demonstrated the feasibility of pre-operative administration in the clinic in adults over age $55^{5}$. The DalCAB showed a PND incidence of $14 \%$, aligning with quantitative reports using extended test batteries ${ }^{6}$.

This ongoing project aims to establish a peri-operative DalCAB that can be administered at home before and after surgery in order to investigate risk factors for PND, as well as monitor the time course of PND and impact on everyday outcomes, by generating a dataset of control and surgical participants 65 years and older. DalCAB results, along with other extensive pre- and intra-operative data will be analyzed using machine-learning methods to establish potential predictive determiners of PND in relation to post-surgical cognitive outcomes.

Methods: Ethics approval was obtained from the local REB. We report data from 86 participants recruited at the QEII Health Sciences Centre surgery pre-admission clinic (Halifax, NS, Canada). 41 surgical participants completed cognitive testing at two time points: first preoperatively, then 45-90 days post-surgery. 45 control (non-surgical) participants completed a similar testing administration schedule. These tests included the DalCAB, the Hospital Anxiety and Depression Scale (HADS), a modified Telephone Interview for Cognitive Status (mTICS), and pain ratings on a visual analogue scale.

Results: Initial analyses examined the prevalence of impaired cognitive function (z score $>1.96$ ) post-operatively in surgical participants in relation to pre-operative testing. Overall, 11/41 (26.8\%) surgical participants had an impairment in at least two cognitive domains, with 4 (7.3\%) showing impairment in 3 domains, including working memory and executive function. Cognitive impairment was not reliably associated with changes in mood or pain. 
Discussion/Conclusion: The at-home, online DalCAB is able to detect post-operative cognitive deficits in surgical patients over the age of 65. Upcoming predictive modelling will identify a patient's predisposition to PND using DaICAB test results, demographics, and health information. In the long run, these factors will be accessible to physicians with an easy-to-use interpretation scale to enhance decision-making and outcomes related to anesthesia delivery methods in this vulnerable population. Empowerment through informed decision-making will ultimately improve patient outcomes.

\section{REFERENCES:}

1. Weiser TG, Regenbogen SE, Thompson KD, Haynes AB, Lipsitz SR, Berry WR, Gawande AA. 2008. An estimation of the global volume of surgery: a modelling strategy based on available data. Lancet. 372:139-44

2. Newman S, Stygall J, Hirani S, Shaefi S, Maze M. 2007. Postoperative cognitive dysfunction after noncardiac surgery: A systematic review. Anesthesiol. 106(3):572-90 3. Steinmetz J, Christensen KB, Lund T, Lohse N, Rasmussen LS. 2009. Long-term consequences of postoperative cognitive dysfunction. Anesthesiol. 110(3):548-55

4. Rasmussen LS, Larsen K, Houx P, Skovgaard LT, Hanning CD, Moller JT, the ISPOCD group. 2001. The assessment of postoperative cognitive function. Acta Anesthesiol Scand. 45(3):275-89

5. Sardiwalla Y, Eskes G, Bernard A, George, RB, Schmidt M. 2018. Assessing the feasibility of using the Dalhousie Computerized Attention Battery to measure postoperative cognitive dysfunction in older patients. J Perioper Prac. 29(10):328-36 
Inter-Individual Variability in Postoperative Worsening of Sleep Disordered Breathing: A Pilot Cohort Study

Margot Klemmer ${ }^{1}$; Eleni Giannouli ${ }^{2}$; Kathleen Clouston ${ }^{3}$; Krista Hardy ${ }^{3}$; Marshall Tenenbein ${ }^{1}$; Robert Balshaw ${ }^{4}$; Thomas Mutter ${ }^{1}$

1 Department of Anesthesiology, Perioperative and Pain Medicine, University of Manitoba, Winnipeg, Canada

2 Department of Internal Medicine, University of Manitoba, Winnipeg, Canada

3 Department of Surgery, University of Manitoba, Winnipeg, Canada

4 George and Fay Yee Centre for Health Care Innovation, University of Manitoba, Canada

Introduction: Increased risk of postoperative complications in obstructive sleep apnea patients is thought to result from the combined negative effects of surgery, anesthetic agents, and opioid analgesics exacerbating upper airway dysfunction. ${ }^{1}$ However, little attention has been directed to inter-individual variation in this effect ${ }^{2}$, even though patients who experience significant postoperative worsening of sleep disordered breathing (SDB) are likely at highest risk of complications. This single centre, pilot cohort study established protocol adherence, described the variability in postoperative changes in SDB among a homogeneous surgical population receiving standardized perioperative care, and explored potential predictors of worsened postoperative SDB.

Methods: Ethics approval was obtained from the local research ethics board. Consented patients having laparoscopic bariatric surgery between May 2017 and September 2018 recorded preoperative and postoperative overnight oximetry studies, using an approved monitor. ${ }^{3} \mathrm{~A}$ trained analyst calculated the oxygen desaturation index (ODI) and severity of nocturnal hypoxemia (cumulative percent of study time with $\mathrm{SpO} 2<90 \%$, CT90) for each study. For each patient, the worst postoperative night was compared to the preoperative baseline for both parameters with changes of $>10$ events $/ \mathrm{h}$ in ODI and $>5 \%$ in CT90 considered clinically significant. Caregivers were blind to oximetry study results. All other care was as per local routine with hospital discharge on the first post-operative morning. Analysis was descriptive except as noted.

Results: Thirty-five of 96 (36\%) of patients had complete oximetry data for analysis (studies in the preoperative period and each of the first three post operative nights). Baseline parameters were not significantly different between patients with and without analyzable data. 15/35 (43\%) had evidence of pre-existing SDB (preoperative ODI>5). Postoperative night 2 was most frequently the worst for both ODI $(21 / 35,60 \%)$ and CT90 (16/35, 46\%). Group median ODI and CT90 changed modestly from preoperative baseline to worst postoperative night $(-0.3$ events/h and $+4.9 \%$, respectively), despite marked interindividual variation (Figure). ODI and CT90 respectively worsened by a clinically significant amount in $4(11 \%)$ and $14(40 \%)$ patients but showed net improvement in $20(57 \%)$ and $8(23 \%)$. Univariate linear regression modelling found body mass index, baseline ODI, early postoperative hypoxemia and opioid analgesic use on post-operative day 1 predicted $(p<0.05)$ worsening CT90 while a history of positive airway pressure device use was protective. Only pre-operative CT90 and male sex predicted worsened post-operative ODI. 
Conclusions: Protocol adherence was comparable to similar studies ${ }^{2}$ but might be improved by simplification of study procedures. Despite similar surgery and perioperative care, we observed considerable inter-individual variability in magnitude and direction of change in postoperative ODI and CT90 that was incompletely explained by pre-existing SDB and other clinical variables. This variability requires further study but may relate to OSA phenotype ${ }^{4}$, susceptibility to opioid induced respiratory depression and other patient related factors.

\section{REFERENCES:}

1. Chung, S. A., Yuan, H. \& Chung, F. A Systemic Review of Obstructive Sleep Apnea and Its Implications for Anesthesiologists. Anesth. Analg. 107, 1543 (2008).

2. Chung, F., Liao, P., Yegneswaran, B., Shapiro, C. M. \& Kang, W. Postoperative Changes in Sleep-disordered Breathing and Sleep Architecture in Patients with Obstructive Sleep Apnea. Anesthesiology 120, 287-298 (2014).

3. Collop, N. A. et al. Clinical guidelines for the use of unattended portable monitors in the diagnosis of obstructive sleep apnea in adult patients. Portable Monitoring Task Force of the American Academy of Sleep Medicine. J. Clin. Sleep Med. JCSM Off. Publ. Am. Acad. Sleep Med. 3, 737-747 (2007).

4. Hillman, D. R. \& Chung, F. Anaesthetic management of sleep-disordered breathing in adults. Respirology 22, 230-239 (2017).
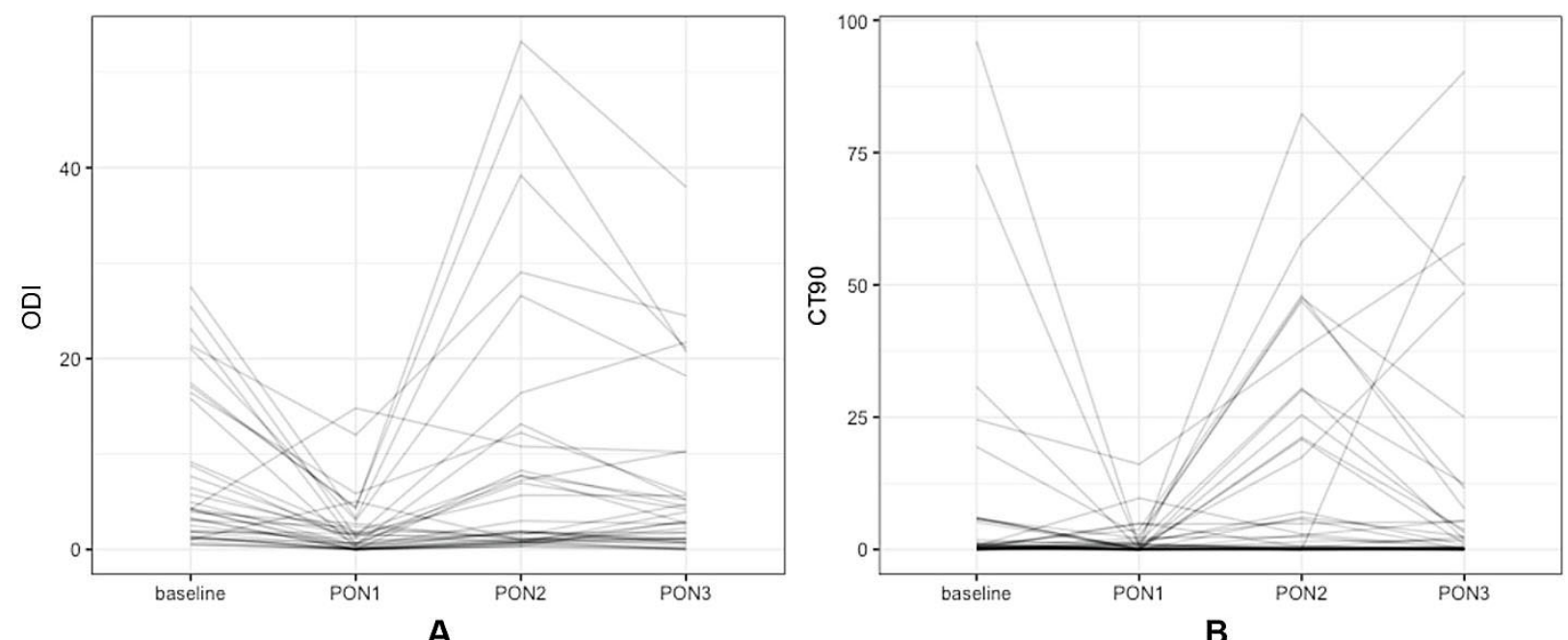

Figure. Spaghetti plots of individual patient's pre- and post-operative $(\mathbf{A})$ oxygen desaturation index (ODI, events/h) and (B) cumulative time spent with $\mathrm{SpO} 2$ of $<90 \%$ (CT90) expressed as a percentage of total recording time. Baseline is the weighted average of up to three pre-operative oximetry studies. PON1, 2, and 3 are postoperative night 1, 2 and 3 oximetry studies, respectively. 


\section{Interventions that Increase Unused Postoperative Opioid Disposal Rates: A Systematic}

\section{Review}

Curtis Van Doormaal ${ }^{1}$; Luke Hartford ${ }^{2}$; Collin Clarke ${ }^{1}$; Patrick Murphy ${ }^{2,3}$

1 Department of Anesthesia and Perioperative Medicine, Schulich School of Medicine and Dentistry, Western University, London, Ontario, Canada.

2 Division of General Surgery, Department of Surgery, Schulich School of Medicine and Dentistry, Western University, London, Ontario, Canada

3 Division of Acute Care Surgery, Department of Surgery, Indiana University School of Medicine, Indianapolis, IN, USA

Introduction: Opioid prescription and misuse is considered an epidemic. Unused postoperative opioids contribute meaningfully to iatrogenic supply of opioids available for misuse, accidental ingestion or diversion. Efforts at curbing the epidemic have been challenging and safe disposal rates of unused opioids has been poorly evaluated while remaining low (Hartford et al., 2019).

Methods: A systematic review of literature was conducted to explore evidence-based strategies to improve disposal of unused post-operative opioids. Ethics approval was not applicable because the study did not involve human or animal research. PUBMED (MEDLINE), EMBASE and COCHRANE from inception to Sept 2018 were searched. Papers were screened independently by two reviewers using pre-defined criteria. Of those full articles, those meeting our inclusion and exclusion criteria were included in the systematic review. Meta-analysis of data was not possible due to significant heterogeneity in study methodology and quality.

Results: Literature search yielded 883 unique sources. After abstract review, 153 were retained for full article review. 119 did not meet inclusion criteria and 34 articles were included in the review. Interventions researched include patient education, drug take-back days, drug kiosks, drug mail back programs and pharmacy return. Few interventions were evaluated as controlled trials and these involved primarily patient education. Patient education consistently increases patients' awareness, but behavioural interventions have not reached clinical significance. State-wide drug take back days or kiosks have been widely implemented in the US demonstrating bulk returns of large volume unused medications (including opioids), however it is uncertain what proportion of total unused opioids are collected. Only a single, small study evaluated drug mail back programs. Pharmacy return is a program widely available in Ontario, however the usage rates are not well evaluated prospectively, and remain largely underutilized. No study evaluated monetary or non-monetary incentive based strategies.

Conclusion: While patient education increases patients' awareness of concerns and drug takeback programs have yielded high absolute returns, there is a paucity of evidence-based interventions to improve unused postoperative opioid disposal. There is a clear opportunity and need to increase opioid disposal rates by exploring novel strategies to complement effective efforts in reducing excess prescribing. 


\section{REFERENCES:}

Hartford LB, Van Koughnett JAM, Murphy PB, Vogt KN, Hilsden RJ, Clarke CF Allen LJ, Gray SD, Parry NG, Gray DK, \& Leslie KA (2019). Standardization of outpatient procedure (STOP) narcotics: A prospective non-inferiority study to reduce opioid use in outpatient general surgical procedures. J Am Coll Surg. 228(1):81-88. 
Optimizing Anesthetic Technique and Postoperative Analgesia in Candidates Undergoing Hip and Knee Arthroplasty

Elroy Campbell ${ }^{1,2}$, Jean Gelinas ${ }^{2,3}$, Scott Wiens $^{4,5}$, Wynand Wessels ${ }^{4}$, Robert Staples ${ }^{1}$, Alika Lafontaine $^{1,2}$, Corne Booysen ${ }^{1}$, Carin Strydom ${ }^{1}$, Charles Boldt ${ }^{1}$, Liam McGowan ${ }^{1}$, Josef Mir ${ }^{1,6}$

1 Department of Anesthesiology, Queen Elizabeth II Hospital, Grande Prairie, Canada

2 Department of Anesthesiology and Pain Medicine, University of Alberta, Edmonton, Canada

3 Department of Anesthesiology, Surrey Memorial Hospital, Surrey, Canada

4 Department of Orthopedics, Queen Elizabeth II Hospital, Grande Prairie, Canada

5 Division of Orthopedic Surgery, University of Alberta, Edmonton, Canada

6 Department of Anesthesia, Dalhousie University, Queen Elizabeth II Health Sciences Centre, Halifax, Canada

Introduction: There are very different approaches across Canada and around the world to the perioperative care of patients undergoing hip or knee arthroplasty. As clinicians, it is difficult to establish "best practice" strategies as we often feel we are operating in the dark and lack data and tools to get feedback on how our management options actually impact outcomes.

Objectives: In order to obtain locally relevant data to inform decisions relating to a best practice approach, we decided to create our own quality improvement toolkit by making use of some innovative modern technology. The idea was to measure a wide range of data about our current practice, analyze it and show results to clinicians, staff and management. That would give us the feedback to identify deficiencies more objectively, adapt our practice and then measure the impact through a continuing process of quality control.

Methods: Ethics approval was obtained from the local REB. To learn as much about our arthroplasty candidates and their perioperative progress as possible, we used five main sources of data: (1) Subjective patient reported outcome and experience measures reported via a tabletbased communication platform with REDCap. (2) Objective mobility data from wrist worn mobility monitors (recording information about movement, sleep, temperature and ambient light). (3) Anesthesiologists completed a simple tablet based electronic REDCap tickform about anesthetic drugs and techniques. (4) A study nurse recorded chart reviews of in-hospital analgesic and anti-emetic use. (5) Physiotherapy performed a preoperative Timed Up and Go (TUG) test. We analyzed all this data relating patient experience over time to patterns of practice over time. We were specifically interested in how pain, nausea, sleep, mobility and their pharmacological treatment interacted to affect patient recovery. We also looked at the ability of measured preoperative mobility to predict post-operative recovery.

Results: The mobility sensors showed fascinating information about changes in mobility and sleep patterns after surgery. The main targets for improvement we identified were pain control, disrupted sleep patterns, an incidence of postoperative nausea of $40 \%$, poor maximization of opioid sparing strategies (under-administration and under-dosing acetaminophen and NSAIDS), a surprisingly high incidence of thigh pain (tourniquet pain) and generally slow recovery of mobility that hardly reaches pre-operative baseline by two weeks. Patients were very poor at estimating their own mobility.

Conclusion: A simple perioperative learning health system using innovative modern technologies gave us fascinating insights into what is actually happening to our arthroplasty 
patients and clear targets for improvement and tools to measure the impact of interventions. Hopefully these tools will be built into future health systems (like EPIC). Mobility should be seen as a fifth vital sign and objectively monitored. This mobility phenotyping is a huge field for future research.

\section{REFERENCES}

1. Glasziou P, Ogrinc G, Goodman S. Can evidence-based medicine and clinical quality improvement learn from each other? BMJ Qual Saf 2011;20 Suppl 1(Suppl 1): i13-7.

2. Timmermans $S$, Mauck $A$. The promises and pitfalls of evidence-based medicine. Health Aff (Millwood) 2005;24(1):18-28.

3. The ideal of continuous improvement. N Engl J Med 1989;320(21):1424-5.

4. Dixon-Woods M, McNicol S, Martin G. Ten challenges in improving quality in healthcare: lessons from the Health Foundation's program evaluations and relevant literature. BMJ Qual Saf 2012;21(10):876-84.

5. Bourne RB, Chesworth BM, Davis AM, Mahomed NN, Charron KDJ. Patient satisfaction after total knee arthroplasty: who is satisfied and who is not? Clin Orthop Relat Res 2010;468(1):57-63.

6. Apfelbaum JL, Chen C, Mehta SS, Gan TJ. Postoperative pain experience: results from a national survey suggest postoperative pain continues to be undermanaged. Anesth Analg 2003;97(2):534-40-tableofcontents.

7. Meissner W, Coluzzi F, Fletcher D, et al. Improving the management of post-operative acute pain: priorities for change. Curr Med Res Opin 2015;31(11):2131-43.

8. Hebl JR, Kopp SL, Ali MH, et al. A comprehensive anesthesia protocol that emphasizes peripheral nerve blockade for total knee and total hip arthroplasty. J Bone Joint Surg Am 2005;87 Suppl 2(suppl_2):63-70.

9. Argenson J-NA, Husted H, Lombardi A, Booth RE, Thienpont E. Global Forum: An International Perspective on Outpatient Surgical Procedures for Adult Hip and Knee Reconstruction. J Bone Joint Surg Am 2016;98(13): e55.

10. Köksal I, Tahta M, Şimşek ME, Doğan M, Bozkurt M. Efficacy of rapid recovery protocol for total knee arthroplasty: a retrospective study. Acta Orthop Traumatol Turc 2015;49(4):3826.

11. Mammoto T, Fujie K, Mamizuka N, et al. Effects of postoperative administration of celecoxib on pain management in patients after total knee arthroplasty: study protocol for an open-label randomized controlled trial. Trials 2016;17(1):45.

12. Korean Knee Society. Guidelines for the management of postoperative pain after total knee arthroplasty. Knee Surg Relat Res 2012;24(4):201-7.

13. Botti M, Kent B, Bucknall T, et al. Development of a Management Algorithm for Postoperative Pain (MAPP) after total knee and total hip replacement: study rationale and design. Implement Sci 2014;9(1):110.

14. Chou R, Gordon DB, de Leon-Casasola OA, et al. Management of Postoperative Pain: A Clinical Practice Guideline from the American Pain Society, the American Society of Regional Anesthesia and Pain Medicine, and the American Society of Anesthesiologists' Committee on Regional Anesthesia, Executive Committee, and Administrative Council. J Pain 2016;17(2):131-57.

15. Practice Guidelines for Acute Pain Management in the Perioperative Setting. 2017:1-26. 
16. Gordon DB, de Leon-Casasola OA, Wu CL, Sluka KA, Brennan TJ, Chou R. Research Gaps in Practice Guidelines for Acute Postoperative Pain Management in Adults: Findings from a Review of the Evidence for an American Pain Society Clinical Practice Guideline. J Pain 2016;17(2):158-66.

17. Bade MJ, Stevens-Lapsley JE. Early high-intensity rehabilitation following total knee arthroplasty improves outcomes. J Orthop Sports Phys Ther 2011;41(12):932-41.

18. Ranawat CS, Ranawat AS, Mehta A. Total knee arthroplasty rehabilitation protocol: what makes the difference? J Arthroplasty 2003;18(3 Suppl 1):27-30.

19. Munin MC, Rudy TE, Glynn NW, Crossett LS, Rubash HE. Early inpatient rehabilitation after elective hip and knee arthroplasty. JAMA 1998;279(11):847-52.

20. Davenport SJ, Arnold M, Hua C, Schenck A, Batten S, Taylor NF. Physical Activity Levels During Acute Inpatient Admission After Hip Fracture are Very Low. Physiother Res Int 2015;20(3):174-81.

21. Basch E. Patient-Reported Outcomes - Harnessing Patients' Voices to Improve Clinical Care. N Engl J Med 2017;376(2):105-8.

22. Ajami S, Bagheri-Tadi T. Barriers for Adopting Electronic Health Records (EHRs) by Physicians. Acta Inform Med 2013;21(2):129-34.

23. Ko V, Naylor JM, Harris IA, Crosbie J, Yeo AET. The six-minute walk test is an excellent predictor of functional ambulation after total knee arthroplasty. BMC Musculoskelet Disord 2013;14(1):145.

24. Bade MJ, Kohrt WM, Stevens-Lapsley JE. Outcomes before and after total knee arthroplasty compared to healthy adults. J Orthop Sports Phys Ther 2010;40(9):559-67.

25. Abujaber SB, Marmon AR, Pozzi F, Rubano JJ, Zeni JA. Sit-To-Stand Biomechanics Before and After Total Hip Arthroplasty. J Arthroplasty 2015;30(11):2027-33.

26. Mizner RL, Petterson SC, Clements KE, Zeni JA, Irrgang JJ, Snyder-Mackler L. Measuring functional improvement after total knee arthroplasty requires both performance-based and patient-report assessments: a longitudinal analysis of outcomes. J Arthroplasty 2011;26(5):728-37.

27. Inoue $\mathrm{Y}$, Kimura $\mathrm{T}$, Noro $\mathrm{H}$, et al. Is laparoscopic colorectal surgery less invasive than classical open surgery? Quantitation of physical activity using an accelerometer to assess postoperative convalescence. Surg Endosc 2003;17(8):1269-73.

28. Inouez $\mathrm{Y}$, Kimura T, Fujita S, et al. A new parameter for assessing postoperative recovery of physical activity using an accelerometer. Surg Today 2003;33(9):645-50.

29. Kleijn LLA, van Hemert WLW, Meijers WGH, et al. Functional improvement after unicompartmental knee replacement: a follow-up study with a performance-based knee test. Knee Surg Sports Traumatol Arthrosc 2007;15(10):1187-93.

30. Cook DJ, Thompson JE, Prinsen SK, Dearani JA, Deschamps C. Functional recoveryin the elderly after major surgery: assessment of mobility recovery using wireless technology. Ann Thorac Surg 2013;96(3):1057-61.

31. Wollmerstedt N, Nöth U, Ince A, Ackermann H, Martell JM, Hendrich C. The Daily Activity Questionnaire: a novel questionnaire to assess patient activity after total hip arthroplasty. J Arthroplasty 2010;25(3):475-480.e1-3.

32. King WC, Belle SH, Eid GM, et al. Physical activity levels of patients undergoing bariatric surgery in the Longitudinal Assessment of Bariatric Surgery study. Surg Obes Relat Dis 2008;4(6):721-8. 


\section{Mobility phenotype of a patient with poor recovery.}

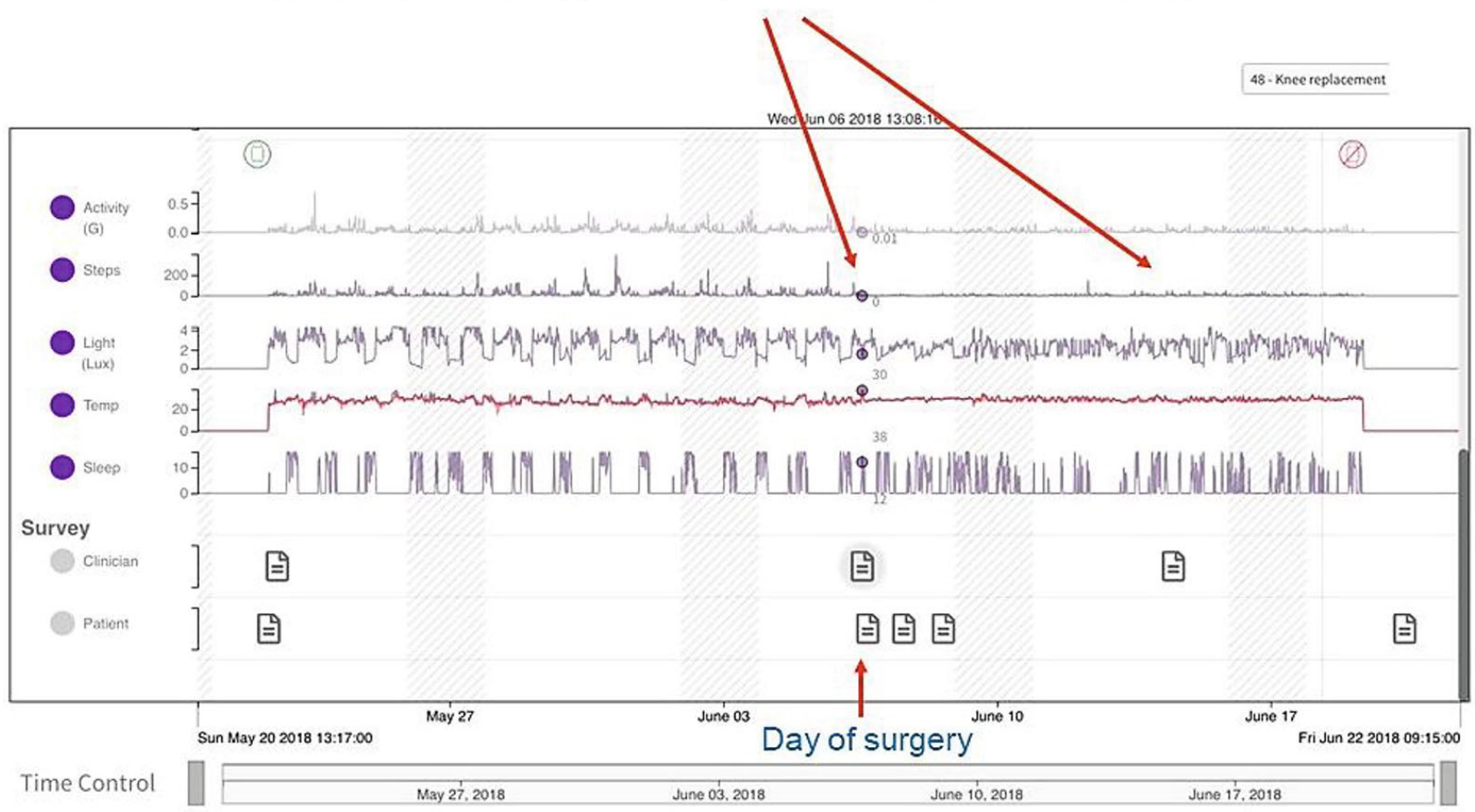

Low activity after surgery, not progressively walking more.

Sleep is very disturbed after surgery and does not seem to be returning to baseline after two weeks. 
Perioperative Outcomes Following Preoperative Epidural Analgesia in Hip Fracture Patients Undergoing Surgical Repair: A Systematic Review and Meta-Analysis

Jonathan Choi ${ }^{1}$; Rachel Lim ${ }^{1}$; Richard Merchant ${ }^{2,3}$; Susan Lee ${ }^{2,3}$

1 Faculty of Medicine, University of British Columbia, Vancouver, Canada

2 Department of Anesthesiology, Pharmacology \& Therapeutics, University of British Columbia, Vancouver, Canada

3 Royal Columbian Hospital, Fraser Health Authority, New Westminster, Canada

Introduction: With an aging population, it is projected that the number of annual hip fractures will increase to 6.26 million by 2050 (1). In the first 3 months after a hip fracture, mortality and healthcare expenditures are high (2-3). The elderly have reduced physiological reserves, increasing their susceptibility to inflammatory cytokines released by trauma, potentially leading to cardiovascular and multi-system complications (4). Through its anti-inflammatory mechanisms, epidurally administered anesthesia may improve perioperative outcomes in hip fracture patients (5). Previous systematic reviews have compared general vs. neuraxial anesthesia for the operative management of hip fractures, but to our knowledge the use of epidural analgesia in the presurgical period has not yet been reviewed $(6,7)$. This systematic review aims to examine the effects of preoperative epidural analgesia on hip fracture patients undergoing surgical repair.

Methods: Ethics approval was not applicable because the study did not involve human or animal research. The study protocol was registered with the PROSPERO systematic reviews register: CRD42019140396. The following databases were searched: MEDLINE. EMBASE, and Cochrane Controlled Register of Trials. Grey literature was hand-searched. Title and abstract screening, full-text review, risk of bias assessment, and data extraction were performed independently by two reviewers. Disagreement was resolved through consultation with a third reviewer. The heterogeneity of the included studies was assessed using the $\mathrm{I}^{2}$ statistic and a random-effects meta-analysis was conducted once sufficient homogeneity was shown $\left(\mathrm{I}^{2}<\right.$ $75 \%)$. An assessment for publication bias was performed. Due to the small number of included studies, funnel plots and Egger's weighted regression method were not used.

Results: 2243 records were identified in the literature search conducted in June 2019. After 473 duplicates were removed, 1770 records were screened by title and abstract. 28 records were assessed for eligibility by full-text and 4 studies (8-11) met all inclusion criteria, which included a total of 221 patients randomized to preoperative epidural analgesia or alternative forms of analgesia. Preoperative epidural analgesia resulted in reduced cardiac events, which was a reported outcome in two included studies. The relative risk of suffering a cardiac event was 0.36 $\left(95 \% \mathrm{Cl} 0.18\right.$ to $\left.0.82 ; I^{2}=0 \%\right)$ favouring those that received preoperative epidural analgesia. For the outcome of postoperative mortality, three studies were included for meta-analysis (the fourth did not report mortality as an outcome). There was no significant reduction in mortality in the group that received preoperative epidural analgesia $\left(\mathrm{RR}=0.44 ; 95 \% \mathrm{Cl} 0.07\right.$ to $2.63 ; 1^{2}=$ $55 \%)$.

Conclusion: Preoperative epidural analgesia for hip fracture likely reduces perioperative cardiac events. Reduced mortality was not demonstrated, but the number of included studies was low. More research should be done to determine the benefit of early epidural analgesia for hip fractured patients. 


\section{REFERENCES:}

1. Cooper C, Campion G, Melton LJ. Hip fractures in the elderly: a world-wide projection. Osteoporosis Int. 1992 Nov;2(6):285-289.

2. Leslie WD, O'Donnell S, Jean S, Lagace C, Walsh P, Bancej C, et al. Trends in hip fracture rates in Canada. JAMA. 2009 Aug 26;302(8):883-889.

3. White S, Moppett I, Griffiths R. Outcome by mode of anaesthesia for hip fracture surgery. An observational audit of 65535 patients in a national dataset. Anaesthesia. 2014;69(3):224-230.

4. Tian-Sheng S, Jian-Zheng Z, Zhi L. Current view on elderly hip fracture and inflammatory response. Austin J Orthopade \& Rheumatol. 2016;3(2):1030.

5. Chloropoulou P, latrou C, Vogiatzaki T, Kotsianidis I, Trypsianis G, Tsigalou C, et al. Epidural anesthesia followed by epidural analgesia produces less inflammatory response than spinal anesthesia followed by intravenous morphine analgesia in patients with total knee arthroplasty. Med Sci Monit. 2013 Jan 28;19:73-80.

6. Van Waesberghe J, Stevanovic A, Rossaint R, Coburn M. General vs. neuraxial anaesthesia in hip fracture patients: a systematic review and meta-analysis. BMC Anesthesiology. 2017;17(1):87.

7. Guay J, Parker MJ, Gajendragadkar PR, Kopp S. Anaesthesia for hip fracture surgery in adults. Cochrane Database of Systematic Reviews. 2016 Feb 22;2:000521.

8. Luger TJ, Kammerlander C, Benz M, Luger MF, Garoscio I. Peridural anesthesia or ultrasound-guided continuous 3-in-1 block: which is indicated for analgesia in very elderly patients with hip fracture in the emergency department? Geriatr Orthop Surg Rehabil. 2012 Sep;3(3):121-8.

9. Matot I, Oppenheim-Eden A, Ratrot R, Baranova J, Davidson E, Eylon S, et al. Preoperative cardiac events in elderly patients with hip fracture randomized to epidural or conventional analgesia. Anesthesiology. 2003 Jan;98(1):156-63.

10. Scheinin H, Virtanen T, Kentala E, Uotila P, Laitio T, Hartiala J, et al. Epidural infusion of bupivacaine and fentanyl reduces perioperative myocardial ischaemia in elderly patients with hip fracture - a randomized controlled trial. Acta Anaesthesiol Scand. 2000 Oct;44(9):1061-70.

11. Temelkovska-Stevanovska M, Trajanka T, Mojsova-Mijovska M, Jovanovski-Srceva M. Perioperative cardiac morbidity in elderly patients with epidural versus systemic analgesia for hip fracture surgery. Mac Med Review. 2014;68(2):93-100. 


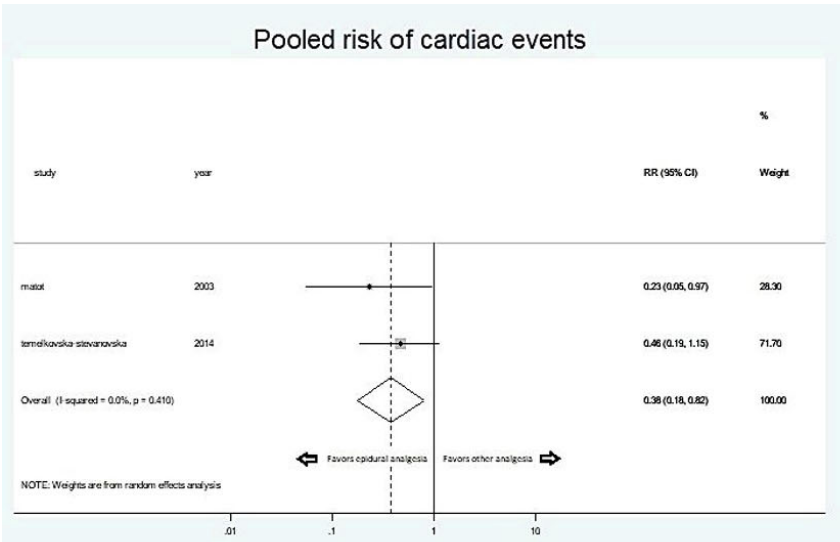

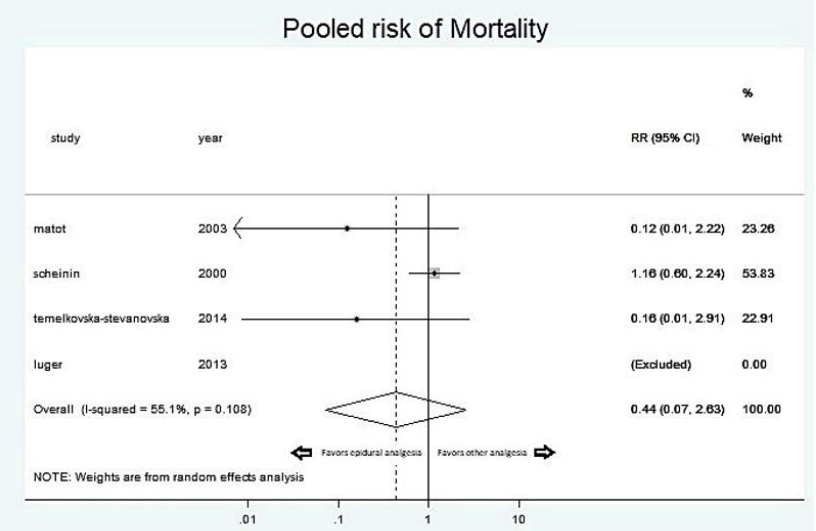




\section{Potentially Low-Value Preoperative Anesthesiology Consult Utilization in Ontario}

Joanna Dion ${ }^{1}$; Robert J. Campbell ${ }^{2}$; Paul Nguyen ${ }^{3}$; Jason A. Beyea ${ }^{3,4}$

1 Department of Anesthesiology and Perioperative Medicine, Queen's University, Kingston, Ontario, Canada

2 Department of Ophthalmology, Queen's University, Kingston, Ontario, Canada

3 ICES Queen's, Queen's University, Kingston, Ontario, Canada

4 Department of Otolaryngology, Kingston Health Sciences Centre, Queen's University, Kingston, Ontario, Canada

Introduction: Physician consultations are a limited resource within the healthcare system. Anesthesiologists in particular have multiple clinical roles, including providing anesthesia during surgery and other procedures, preoperative clinic appointments to prepare patients for surgery, and providing postoperative care to patients. Time spent in one of these roles is time they are not available for the other roles. This study sought to evaluate current consultation usage patterns, with an objective to determine opportunities for more efficient utilization.

Methods: Ethics approval was obtained from the local REB. A retrospective comprehensive population-based cohort study was performed, evaluating all hospitals in the Canadian province of Ontario from 2002-2018. The main outcome measures were American Society of Anesthesiologists (ASA) classification of the patients, and whether the patients underwent surgery within 3 months following the anesthesia consultation.

Results: A cohort of 2,023,499 patients, and a total of 2,920,100 preoperative anesthesia consultations was obtained. The number of consults per year doubled between 2003 (112,983/year) and 2017 (246,427/year). Each year, an average of $19.32 \%$ of the consults (range: 17.69-20.49\%) were for patients that did not progress to having surgery. Of those that did have surgery following the anesthesia consult, $37.23 \%$ were ASA Classification I or II. The most common surgical procedures (percent of total) following anesthesia consult were: Knee implantation of internal device $(9.46 \%)$, hip implantation of internal device $(5.84 \%)$, cataract excision $(4.09 \%)$, repair of muscle of chest/abdomen $(3.31 \%)$, uterus excision $(2.76 \%)$, and gallbladder excision (2.67\%).

Interpretation: This study reveals important data relating to the utilization and trends over time of preoperative anesthesia consultations. Opportunities for optimization were found, including patients who did not proceed to surgery, and those healthier patients that may not require this consultation.

\section{REFERENCES:}

https://www.cihi.ca/en/health-spending/2018/national-health-expenditure-trends/how-has-healthspending-growth-changed-over-the-last-40-years

https://www.cihi.ca/en/health-spending/2018/national-health-expenditure-trends/where-is-mostof-the-money-being-spent-in-health-care-in-2018 
Canadian Institute for Health Information, Inpatient hospitalizations: Voumes, Lengths of Stay, and Standardized Rates, QuickStats.

Ontario Hospital Association, QuickFacts 2018: Ontario's Public Hospitals. Available from: https://www.oha.com/your-hospitals

Abouleish AE, Leib ML, Cohen NH. ASA provides examples to each ASA physical status class. ASA Monitor 2015; 79:38-9 http://monitor.pubs.asahq.org/article.aspx?articleid=2434536

Beyea JA, Rosen E, Stephens T, Nguyen P, Hall SF. Audiometric Testing Guideline Adherence in Children Undergoing Tympanostomy Tubes: A Population-Based Study. Otolaryngol Head Neck Surg. 2018;158:1127-1133.

Duceppe E, Parlow J, MacDonald P, Lyons K, McMullen M, et. al. Canadian Cardiovascular Society Guidelines on Perioperative Cardiac Risk Assessment and Management for Patients Who Undergo Noncardiac Surgery. Canadian Journal of Cardiology. 2017;33(1):17-32.

Farasatkish R, Aghdaii N, Azarfarin R, Yazdanian F. Can preoperative anesthesia consultation clinic help to reduce operating room cancellation rate of cardiac surgery on the day of surgery? Middle East J Anaesthesiol. 2009;20:93-6.

Ferschl MB, Tung A, Sweitzer B, Huo D, Glick DB. Preoperative clinic visits reduce operating room cancellations and delays. Anesthesiology. 2005;103:855-9.

Halm EA, Browner WS, Tubau JF, Tateo IM, Mangano DT. Echocardiography for assessing cardiac risk in patients having noncardiac surgery. Study of Perioperative Ischemia Research Group. Ann Intern Med. 1996;125:433-41.

Hawker GA, Coyte PC, Wright JG, Paul JE, Bombardier C. Accuracy of administrative data for assessing outcomes after knee replacement surgery. J Clin Epidemiol. 1997;50:265-73.

Kirkham KR, Wijeysundera DN, Pendrith C, Ng R, Tu JV, Laupacis A, Schull MJ, Levinson W, Bhatia RS. Preoperative testing before low-risk surgical procedures. CMAJ. 2015;187:E349E358.

Kirkham KR, Wijeysundera DN, Pendrith C, Ng R, Tu JV, Boozary AS, Tepper J, Schull MJ, Levinson W, Bhatia RS. Preoperative Laboratory Investigations: Rates and Variability Prior to Low-risk Surgical Procedures. Anesthesiology. 2016;124:804-14.

Klopfenstein CE, Forster A, Van Gessel E. Anesthetic assessment in an outpatient consultation clinic reduces preoperative anxiety. Can J Anaesth. 2000;47:511-5.

Levinson W, Huynh T. Engaging physicians and patients in conversations about unnecessary tests and procedures: Choosing Wisely Canada. CMAJ. 2014;186:325-6.

Pollard JB, Garnerin P, Dalman RL. Use of outpatient preoperative evaluation to decrease length of stay for vascular surgery. Anesth Analg. 1997;85:1307-11. 
Thilen SR, Treggiari MM, Lange JM, Lowy E, Weaver EM, Wijeysundera DN.

Preoperative consultations for medicare patients undergoing cataract surgery. JAMA Intern Med. 2014;174:380-8.

Thilen SR, Wijeysundera DN, Treggiari MM. Preoperative Consultations. Anesthesiol Clin. 2016;34:17-33.

van Klei WA, Moons KG, Rutten CL, Schuurhuis A, Knape JT, Kalkman CJ, Grobbee DE. The effect of outpatient preoperative evaluation of hospital inpatients on cancellation of surgery and length of hospital stay. Anesth Analg. 2002;94:644-9.

Wijeysundera DN. Preoperative consultations by anesthesiologists. Curr Opin Anaesthesiol. 2011 Jun;24:326-30.

Wijeysundera DN, Austin PC, Beattie WS, Hux JE, Laupacis A. A population-based study of anesthesia consultation before major noncardiac surgery. Arch Intern Med. 2009;169:595-602.

Wijeysundera DN, Austin PC, Beattie WS, Hux JE, Laupacis A. Variation in the practice of preoperative medical consultation for major elective noncardiac surgery: a population-based study. Anesthesiology. 2012;116:25-34. 


\section{Pre-Surgery Glucose and Metabolic Prehabilitation (Sugar-I Follow-Up Study)}

James Jiang ${ }^{1}$; Aihua $\mathrm{Wu}^{2,3}$

1 Hospital Medical Officer, Eastern Health, VIC 3135, Australia

2 Department of Anesthesia and Pain Management, Maroondah Hospital, Eastern Health, VIC 3135, Australia

3 Department of Anesthesia and Perioperative Medicine, Faculty of Medicine, Nursing and Health Sciences, Monash University, VIC 3800, Australia

Background: Our Sugar-I study indicated that patients' compliance to preoperative fasting guidelines, particularly to clear fluids, was poor [1]. Since then, local fasting protocols have been revised, focusing on patient and staff education on current clear fluids fasting guidelines. This follow-up study aims to determine whether such education improved patients' adherence to fasting guidelines.

Methods: Patient education was provided via revised written fasting instructions which were sent to all elective surgical patients. The instructions clearly defined specific fasting times for clear fluids and solid foods and, included examples of acceptable and unacceptable clear fluids. Staff education was provided through Inservice meetings, consisting of content similar to the revised written instructions.

After institutional ethics approval, medical records for elective surgical patients during a 3-month period were then reviewed, and fasting times for fluids and solids were extracted. Fasting categories were defined as per the initial study [1].

Results: Data from 1852 patients were examined and analysed.

The results indicated that still too many patients fasted for too long, $48 \%$ for clear fluids and $55 \%$ for solid foods.

When compared to the initial study [1], a significant improvement was seen in the acceptable fasting category for clear fluid, $49 \%$ versus $6 \%$. However, the rate of acceptable fasting for solid foods only slightly increased to $43 \%$ from $39 \%$.

Of concern, while non-fasted patients from solid foods remained similar (2\%), non-fasting from clear fluids increased by $3 \%$ in the current study. The significance of such increases in our perioperative practice and the implications on the way we educate our patients and staff requires further exploration.

Conclusions: Our initial measure of revising local fasting protocols to educate patients and staff proved effective, having improved patients' compliance to the current fasting guidelines significantly. However, there was an increase in patients who did not fast from clear fluids properly after receiving education. Thus, further revisions, incorporating solid foods and alternate methods of fasting instruction delivery are needed to address these identified issues. 


\section{REFERENCES:}

1. Thomson A, Wu A. Surgical Metabolic Prehabilitation: a Current Concept of Preoperative Fasting (Sugar Study - I). Anaesth Intensive Care 2017, 45 (1): 117-118. 


\section{Prehabilitation to Improve the Perioperative Functional Trajectory in Major Cancer Surgery}

Enrico Maria Minnella ${ }^{1}$; Amal Bessissow ${ }^{2}$; A. Sender Liberman ${ }^{3}$; Gabriele Baldini ${ }^{1}$; Francesco Carli F.

1 Department of Anesthesia, McGill University Health Centre, Montreal, QC, Canada 2 Department of Medicine, McGill University Health Centre, Montreal, QC, Canada 3 Department of Surgery, McGill University Health Centre, Montreal, QC, Canada

Background: Functional capacity has a strong role in peri-operative medicine, from the moment of decision to operate until full recovery. ${ }^{1}$ Patient-centered outcomes, such as time to return to physical functioning, can impact not only on quality of life, but also on access and continuity of cancer care. ${ }^{2}$ By targeting malnutrition, poor physical capacity and mental distress as key modifiable risk factors, prehabilitation is a preoperative intervention that aims to make patients fitter for surgery, and accelerate their recovery.

Despite mounting evidence of its efficacy, current investigations are deemed to lack of large cohort validation.

Methods/Results: Ethics approval was obtained from the local REB. Pooled data from research trials on prehabilitation in patients undergoing elective non-metastatic colorectal cancer surgery from 2009 to 2016 were analyzed. Patients were grouped into PREHAB or standard of care (CONTROL). All groups received enhanced recovery program. The primary outcome was the change in perioperative physical capacity compared with baseline measurement, evaluated with repeated 6-minute walk tests (6MWT) over a 3-month period.

A total of 397 patients were included, 259 in PREHAB an 138 in the CONTROL. Duration of prehabilitation was 35 days (SD 18). Groups characteristics were well-balanced, patients' mean age 69 years (SD 11.5), 29\% stage II and 27\% stage III cancer. Baseline 6MWT was $428.5 \mathrm{~m}$ (125) in PREHAB, and 417.6 (123.7) in CONTROL, $P=0.410$. Functional trajectories are presented in figure 1. Compared with baseline 6MWT, patients in PREHAB gained $26.3 \mathrm{~m}$ (SD 59.0) preoperatively, while CONTROL dropped by $3.2 \mathrm{~m}$ (SD 63.2), $\mathrm{P}<0.001$. At 4 weeks after surgery; PREHAB dropped by $1.9 \mathrm{~m}$ (SD 69.4), while CONTROL dropped by $50.9 \mathrm{~m}$ (SD 107.2), $\mathrm{P}<0.001$. At 8 weeks after surgery, PREHAB gained $24.9 \mathrm{~m}$ (SD 50.4) while CONTROL dropped -1.9 (DSD 69.4), $\mathrm{P}<0.001$.

Conclusion: Prehabilitation positively modulates for better perioperative functional trajectory of patients undergoing colorectal cancer surgery, and therefore could have a key role in perioperative medicine.

\section{REFERENCES:}

1. Br J Anaesth. 2017 Dec 1;119(suppl_1):i34-i43.

2. Anaesthesia. 2019 Jan;74 Suppl 1:3-6. doi: 10.1111/anae.14502. 
Abstracts

S343


181

An Springer 


\section{Survey on Preoperative Fasting: Patient Experience and Understanding (Sugar-II Follow- up Study)}

James Jiang ${ }^{1}$; Aihua $\mathrm{Wu}^{2,3}$

1 Hospital Medical Officer, Eastern Health, VIC 3135, Australia

2 Department of Anesthesia and Pain Management, Maroondah Hospital, Eastern Health, VIC

3135, Australia

3 Department of Anesthesia and Perioperative Medicine, Faculty of Medicine, Nursing and Health Sciences, Monash University, VIC 3800, Australia

Background: The current guidelines for preoperative fasting recommend that patients should consume a sugary clear fluid up until 2 hours prior to anaesthetics. However, our Sugar-I study indicated that patients' compliance was poor [1]. The initial Sugar-II study [2] was subsequently conducted to investigate the reasons for poor compliance from the patient's perspective, and suggested some misunderstandings of the recommended fasting periods and what constituted a 'clear fluid' [2]. Based on these results, fasting instructions across local institute have been revised, focusing on patient education regarding clear fluids. This follow-up study aims to determine how effective patient education is on improving patients' adherence to the fasting guidelines, understanding of the instructions provided and their experience during the preoperative fasting period.

Methods: After local ethics approval, revised fasting instructions, using specific and straightforward language, were sent to all elective surgical patients.

During a two-month period, patients were randomly selected and followed up after their discharge from recovery room. When sufficiently alert, they were asked to consent and complete an anonymous questionnaire survey as used in the previous study.

Results: Data from 165 patients were examined and compared with the initial study [1].

The results for adherence to the fasting guidelines indicated an improvement for both clear fluids and solid foods, particularly clear fluids, where the acceptable rate was increased by $20 \%$ (from $39 \%$ to $59 \%$ ).

There was a $10 \%$ drop in patients who experienced preoperative discomfort from fasting (from $84 \%$ to $74 \%$ ). The incidence of thirst reduced from $60 \%$ to $44 \%$, however, hunger slightly worsened by $2 \%$ up to $38 \%$.

While there was minimal change in patients' understanding of recommended fasting periods for solid foods, prolonged or excessive fasting from clear fluids was significantly reduced, decreasing by over $50 \%$.

Of note, recognition of 'clear fluids' as inclusive of clear sugary beverages improved dramatically from $3-15 \%$ to $36-62 \%$, although identification of inappropriate beverages (e.g. white coffee or tea) as being 'clear fluids' also increased. 
Compared to the initial study, the revised fasting instructions were reported to be much easier to follow; $10 / 10$ ratings jumped from $26 \%$ to $56 \%$. Similarly, many more patients were satisfied with their fasting experience, with an increase in $10 / 10$ ratings from $8 \%$ to $44 \%$.

Conclusions: Given the diversity of backgrounds, patient education remains notoriously challenging. However, patient comprehension of the fundamentals of the current fasting guidelines through lay language, such as in our study, could be a simple but effective approach to improve their compliance to the fasting instructions, as well as their experience and satisfaction with preoperative fasting.

\section{REFERENCES:}

1. Thomson A, Wu A. Surgical Metabolic Prehabilitation: a Current Concept of Preoperative Fasting (Sugar Study - I). Anaesth Intensive Care 2017, 45 (1): 117-118.

2. Chan P, Wu A. Survey on Preoperative Fasting: Patient Experience and Understanding (Sugar Study - II). ASA NSC 2019. 
The Accuracy of Patient Self-Reported Frailty Using the Clinical Frailty Scale

DesAutels $\mathrm{K}^{1}$; Hladkowicz $\mathrm{E}^{2}$; Beasley $\mathrm{E}^{2}$; Bryson $\mathrm{GL}^{1,2,3}$; Gagné $\mathrm{S}^{1,3}$; Huang $\mathrm{A}^{1,2,3}$; Joanisse $\mathrm{J}^{4}$; Moloo $\mathrm{H}^{1,3}$; Lavallée $\mathrm{L}^{1,2,3}$; MacDonald $D^{5}$; Lalu $\mathrm{M}^{1,2,3}$; Mclsaac DI $\mathrm{I}^{1,2,3}$

1 University of Ottawa, Ottawa, Canada

2 Ottawa Hospital Research Institute, Ottawa, Canada

3 The Ottawa Hospital, Ottawa, Canada

4 Hôpital Montfort, Ottawa, Canada

5 Dalhousie University, Halifax, Canada

Introduction: Frailty is a syndrome related to accumulation of age- and disease-related deficits.1Frailty predicts a $>2$-fold increase in postoperative morbidity, mortality, and patientreported adverse outcomes. ${ }^{2}$ Identification of frailty can direct clinical care that may reduce rates of postoperative mortality. ${ }^{3}$ Guidelines recommend routine preoperative frailty assessment in people $>65$ years, ${ }^{4}$ however, adherence is minimal. Patients can self-report major comorbidities with strong to moderate agreement with a physician history, ${ }^{5}$ however, the accuracy with which patients can evaluate their own frailty status before surgery is unknown. We hypothesized that patients could accurately report their own frailty status; this could empower patients to support an evidence-based approach to their own care.

Methods: Ethics approval was obtained from the local REB. This was an OHSN-REB (20150342) approved sub-study of a multicenter prospective cohort study of people $>65$ years having elective, major noncardiac surgery. During preoperative assessment, consecutive patients (blinded to clinically determined frailty score) self-rated their own frailty score on the Clinical Frailty Scale (CFS). Trained research assistants (blinded) performed formal CFS assessments that were reviewed by the primary investigator. Agreement between self- and clinically-applied CFS scores were assessed using weighted kappa statistics. Difference plots were constructed according to the methods of Bland and Altman. Pearson correlation coefficients were calculated. Explained variance was measured using linear regression. We evaluated whether self- or clinically-applied CFS scores were more accurate in predicting the occurrence of death or new disability 90 -days after surgery.

Results: Five hundred and thirty-one participants had self- and clinically-applied CFS scores. Mean age was 73 (SD6) years, 261 (49.2\%) were female, and 276 (52.0\%) had orthopedic surgery. The median self-rated CFS score was 3 (IQR2-3), the median clinically-applied CFS score was 3 (IQR 2-4). Agreement between self- and clinically-applied CFS was moderate (weighted kappa $=0.57(95 \% \mathrm{Cl} 0.53-0.63)$. Bland-Altman plot is shown in Figure $1 ; 76 \%$ of measurements were within the limits of agreement. Correlation was high $(\rho=0.76, P<0.0001)$ and the majority of the variance in the clinically-applied CFS was explained by the self-CFS $(R 2=0.58)$. When predicting death or new disability, the clinically-applied CFS had increased discrimination and was more strongly associated with the outcome than the self-applied CFS (AUC 0.69 vs 0.67 , Type III Wald P-value 0.046 vs 0.121).

Conclusion: Patients can self-report their own frailty status using the CFS with good agreement and strong correlation to a clinically applied frailty assessment. Interestingly, this level of agreement is similar to that between different anesthesiologists assigning ASA scores to the same patient. However, in our data, clinically applied frailty scores were significantly asconnistan with postoperative outcomes while self-reported scores were not. 


\section{REFERENCES:}

1. Rockwood K. CMAJ 2005;173(5):489-95.

2. Lin H. BMC Geriatrics 2016;16(1):157.

3. Hall D. JAMA Surg 2017;152(2):175-182.

4. Chow B. JACS 2012;215(4), 453-466.

5. Neufeld J. CJA 2014.

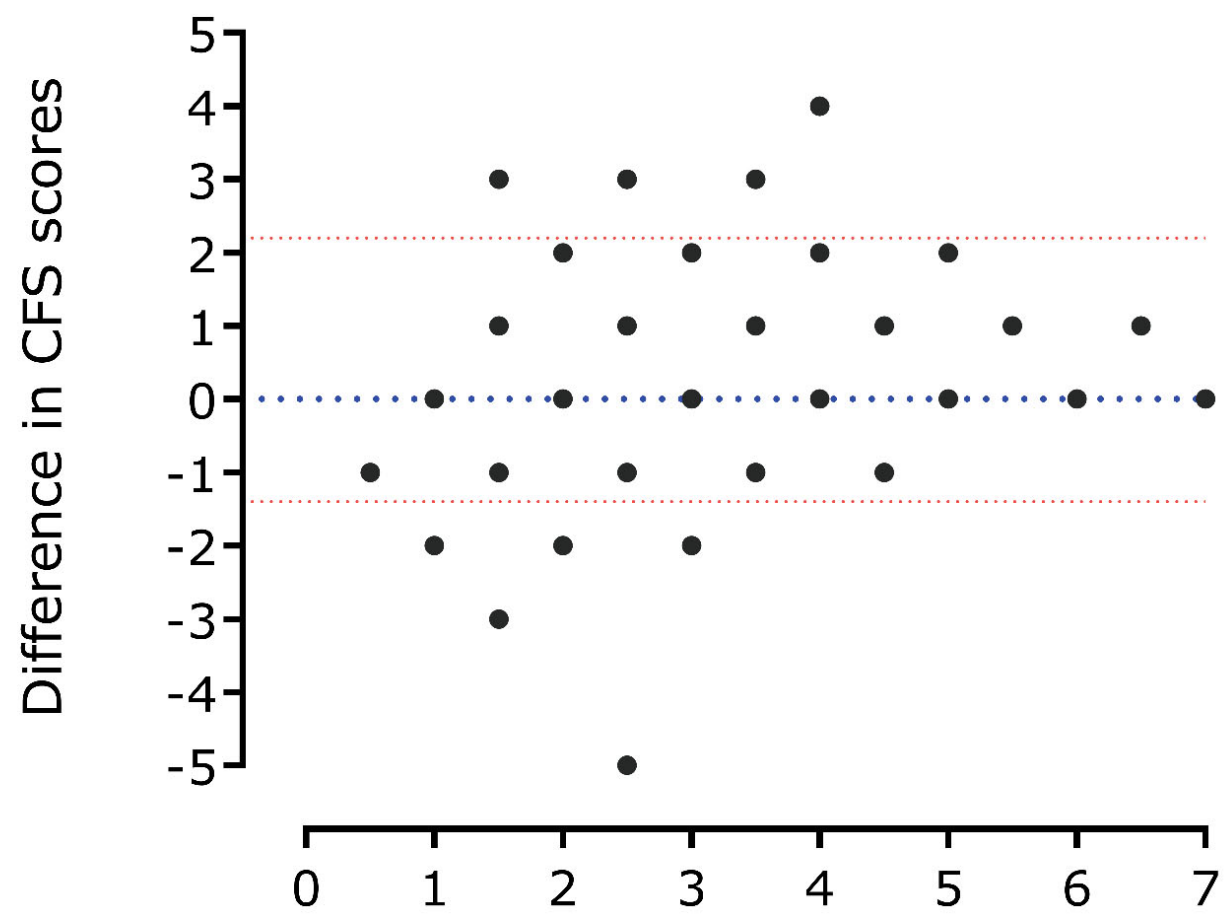

Average CFS score 


\section{PHARMACOLOGY}

\section{Long-Lasting Sensory Block Produced by Quaternary Lidocaine Derivatives: Efficacy and Safety of QX-572, QX-222, and QX-314 in Mice}

Desmond Fung ${ }^{1}$; Hye-Young $\mathrm{Kim}^{2}$; Ian Welch ${ }^{3,4}$; Catherine Schuppli ${ }^{3}$; Aaron Shapiro ${ }^{4,5}$; Sergei Likhodi $^{4,5}$; Bernard MacLeod ${ }^{1}$; Ernest Puil'; Stephan Schwarz ${ }^{1,6}$

1 Hugill Anesthesia Research Centre, Department of Anesthesiology, Pharmacology \& Therapeutics, The University of British Columbia, Vancouver, BC, Canada

2 Department of Anesthesiology and Pain Medicine, College of Medicine, Konkuk University, Chungju Hospital; 82, Chugwon-Daero; Chungju-Si; Chungcheongbuk-Do, 27376, South Korea 3 Centre for Comparative Medicine, The University of British Columbia, Vancouver, BC, Canada 4 BC Provincial Toxicology Centre, Provincial Health Services Authority, Vancouver, BC, Canada

5 Department of Pathology and Laboratory Medicine, The University of British Columbia, Vancouver, BC, Canada

6 Department of Anesthesia, St. Paul's Hospital/Providence Health Care, Vancouver, BC, Canada

Introduction: QX-314, one of several quaternary derivatives of lidocaine, produces long-lasting sensory blockade with a delayed onset in animals. ${ }^{1 ; 2}$ These findings indicate a potential of quaternary compounds as long-lasting local anesthetics. However, subsequent animal studies on intrathecal and systemic administration have raised concerns about toxicity due to QX-314. ${ }^{3 ;}$ ${ }^{4}$ Here, we sought to explore the comparative actions of two other quaternary lidocaine derivatives, QX-572 and QX-222, whose efficacy and toxicity profiles for long-lasting sensory blockade have not been studied. We hypothesized that QX-572 and QX-222, similar to QX-314, produce long-lasting sensory blockade and possess more favorable toxicity profiles.

Methods: Ethics approval was received from the local ACC. We conducted a randomized, double-controlled, and blinded in vivo study in female CD-1 mice. To assess sensory blockade, we used a modified tail flick assay ${ }^{5}$ to record tail flick latencies (TFLs) to thermal noxious stimuli ( $50{ }^{\circ} \mathrm{C}$ water). Mice were injected with $40 \mu \mathrm{L}$ of test solution at the base of the tail. Tail immersion was repeatedly performed to determine sensory blockade (defined as a TFL > 4 s) duration (each concentration, $n=8$ ). To assess local tissue toxicity, tails were collected $24 \mathrm{~h}$ post-injection, sectioned, hematoxylin and eosin stained, and prepared for histopathological analyses. Additionally, to quantify systemic absorption, we developed, validated, and utilized a novel liquid chromatography tandem mass spectrometry (LC-MS/MS) method. For analysis, we log-transformed time-to-event data and used ANOVA with Dunnett's post-hoc multiple comparisons test; we calculated potencies from concentration-response curves fitted using nonlinear regression.

Results: QX-572 (at $\geq 70 \mathrm{mM}$ ) and QX-222 (at $\geq 280 \mathrm{mM}$ ) concentration-dependently produced sensory blockade similar to QX-314 ( $\geq 70 \mathrm{mM}$ ), with a delay to onset compared to lidocaine (70 $\mathrm{mM}[\sim 2 \%])$. The order of potency was QX-572 > QX-314 > QX-222. All quaternary compounds concentration-dependently produced tissue discoloration, edema and scarring at the site of injection (QX-572, $\geq 70 \mathrm{mM}$; QX-314; $\geq 140 \mathrm{mM}$; and QX-222, $\geq 560 \mathrm{mM}$ ). Histopatholoaical analyses revealed myofiber degeneration and inflammation. QX-314 at $\geq 140 \mathrm{mM}$ and 
at $560 \mathrm{mM}$, but not QX-572 (max., $280 \mathrm{mM}$ ), produced systemic toxicity that manifested as death. LC-MS/MS indicated a higher magnitude of systemic absorption following peripheral administration of QX-314 and QX-222 compared to lidocaine.

Conclusion: Similar to QX-314, QX-572 and QX-222 produced long-lasting sensory blockade with a delay to onset compared to lidocaine. QX-572 produced no apparent systemic toxicity but its lowest effective concentration was associated with local tissue toxicity; higher concentrations produced irreversible blockade. QX-314 and QX-222 at high concentrations produced local and, counterintuitively, systemic toxicity. While these results suggest a potential for quaternary compounds as long-lasting LAs, toxicity associated with differences in chemical structures is of concern and warrants further study.

\section{REFERENCES:}

1. Lim TK, Macleod BA, Ries CR, Schwarz SK. The quaternary lidocaine derivative, qx-314, produces long-lasting local anesthesia in animal models in vivo. Anesthesiology 2007;107:305-11.

2. Ries CR, Pillai R, Chung CC, Wang JT, MacLeod BA, Schwarz SK. Qx-314 produces longlasting local anesthesia modulated by transient receptor potential vanilloid receptors in mice. Anesthesiology 2009;111:122-6.

3. Schwarz SK, Cheung HM, Ries CR, Lee SM, Wang JT, MacLeod BA. Lumbar intrathecal administration of the quaternary lidocaine derivative, qx-314, produces irritation and death in mice. Anesthesiology 2010;113:438-44.

4. Cheung HM, Lee SM, MacLeod BA, Ries CR, Schwarz SK. A comparison of the systemic toxicity of lidocaine versus its quaternary derivative qx-314 in mice. Can J Anaesth 2011;58:443-50.

5. Grant GJ, Zakowski MI, Vermeulen K, Langerman L, Ramanathan S, Turndorf H. Assessing local anesthetic effect using the mouse tail flick test. $J$ Pharmacol Toxicol Methods 1993;29:223-6. 
Stability of Reconstituted Remifentanil in Normal Saline Polyvinyl Chloride Bags Stored at Either Room Temperature or $4^{\circ} \mathrm{C}$

Taylor Bucyk ${ }^{1}$; Arianne Fielding ${ }^{1}$; Anna Taylor²; Rakesh Sondekoppam³

1 Department of Anesthesiology and Pain Medicine, University of Alberta, Edmonton, Alberta, Canada

2 Department of Pharmacology, University of Alberta, Edmonton, Alberta, Canada

3 Department of Anesthesia, University of lowa Carver College of Medicine, lowa City, lowa, USA

Introduction: Remifentanil, a potent synthetic opioid, is reconstituted and diluted to the desired concentration. The manufacturer recommends that reconstituted remifentanil should be disposed of after 24 hours, yet limited data exists regarding the drug's long-term stability. Bevans-Warren et al. recently showed that remifentanil mixed to $100 \mu \mathrm{g} / \mathrm{mL}$ in plastic syringes stored at room temperature (RT) is stable at 45 days ${ }^{1}$. Here, we investigate the stability of remifentanil diluted to $10 \mu \mathrm{g} / \mathrm{mL}$ in $0.9 \%$ sodium chloride PVC bags at both RT and $4^{\circ} \mathrm{C}$ over 30 days.

Methods: Ethics approval was not applicable because the study did not involve human or animal research. $1 \mathrm{mg}$ remifentanil vials were reconstituted with $1 \mathrm{~mL}$ of sodium chloride $0.9 \%$ and then diluted to $10 \mu \mathrm{g} / \mathrm{mL}$ in $100 \mathrm{~mL} 0.9 \%$ sodium chloride PVC bags. Bags were stored at either RT $\left(23-25^{\circ} \mathrm{C}\right.$ ) or $4^{\circ} \mathrm{C}$ (3 bags per group). $1 \mathrm{~mL}$ aliquots were collected for analysis at 0 hours, 24 hours, 48 hours, and 30 days and frozen at $-80^{\circ} \mathrm{C}$.

Samples were analyzed via liquid chromatography with tandem mass spectrometry (LC/MS/MS). Remifentanil ( $100 \mu \mathrm{g} / \mathrm{mL}$ in methanol) and fentanyl $(1 \mathrm{mg} / \mathrm{mL}$ in methanol) were used to obtain calibration standards over the range $0.02 \mathrm{ng} / \mu \mathrm{L}$ to $1 \mathrm{ng} / \mu \mathrm{L}$ remifentanil. $2 \mu \mathrm{L}$ of sample solution was injected onto an Agilent Poroshell $1202.7 \mu \mathrm{m} \mathrm{SB}-\mathrm{C} 18$ column at a flow rate of $0.5 \mathrm{~mL} / \mathrm{min}$ using a gradient of $90 \%$ water $(0.1 \%$ formic acid) $10 \%$ acetonitrile $(0.1 \%$ formic acid). Chromatogram peak areas were used to determine the concentration of remifentanil in each sample.

Data was analyzed using mixed effects modelling (restricted maximum likelihood approach) for within group and between group comparisons with time and temperature as factors. Percent remifentanil degradation was calculated with the starting concentrations as the baseline. The product was deemed stable if there was $<10 \%$ degradation ${ }^{2}$.

Results: The reconstituted remifentanil showed a significant difference in measured concentrations in both groups over time $(p=0.001)$. There was also a significant difference in measured concentrations at baseline [MD $(95 \% \mathrm{Cl}): 0.017(0.005,0.03)](p=0.007)$ but not at other time points between group comparison. The percentage decline in remifentanil concentration was significantly higher at 30 days in RT $(-22.47 \%)$ compared to $4^{\circ} \mathrm{C}(+5.6 \%)$, but not at 24 or 48 hour timepoints where they did not show any significant degradation (RT: $24 \mathrm{~h}$ $\left.+19.40 \%, 48 \mathrm{~h}+5.25 \% ; 4^{\circ} \mathrm{C}: 24 \mathrm{~h}+8.85 \%, 48 \mathrm{~h}+4.19 \%\right)$.

Conclusions: Mixing and dilution of remifentanil by the anesthesiologist may result in inaccurate baseline concentrations when employing routine reconstitution methods in the operating room. Reconstituted remifentanil stored at room temperature may show similar 
stability to that stored at $4^{\circ} \mathrm{C}$ but showed significant degradation when stored long term (30 days). This has important environmental and economic implications to practice ${ }^{3}$.

\section{REFERENCES:}

1. Bevans-Warren TS, Clegg DOJ, Sakata DJ, Reilly CA. Remifentanil Stability. Anesth Analg. 2018;127(3):e51. doi:10.1213/ANE.0000000000003600

2. Jansen JJ, Oldland AR, Kiser TH. Evaluation of phenylephrine stability in polyvinyl chloride bags. Hosp Pharm. 2014;49(5):455-457. doi:10.1310/hpj4905-455

3. Richmond EK, Rosi EJ, Walters DM, et al. A diverse suite of pharmaceuticals contaminates stream and riparian food webs. Nat Commun. 2018;9(1):1-9. doi:10.1038/s41467-01806822-w 
The Comparison Effect of Intravenous Injection of Dexmedetomidine, Ondansetron and Pethidine on Postoperative Shivering Among Patients Under Abdominal Surgery

Behzad Nazemroaya ${ }^{1}$, Mitra Jabalameli ${ }^{1}$, Mahsa Heydari ${ }^{2}$, Marzie Taghvaie ${ }^{3}$

1 Department of Anesthesiology and Critical Care, Isfahan University of Medical Sciences, Isfahan, Iran

2 School of Medicine, Isfahan University of Medical Sciences, Isfahan, Iran

3 Shahreza Amiralmomenin hospital, Isfahan University of Medical Sciences, Isfahan, Iran

Introduction: Postoperative shivering is a common complication of various surgical, so far no acceptable theory has been presented on the prevention of it. The aim of this study was to compare the effect of intravenous injection of, Dexmedetomidine, ondansetron and Pethidine on postoperative Shivering among patients under Abdominal Surgery.

Methods: Ethics approval was obtained from the ethics committee of Isfahan University of Medical Sciences. (IR.MUI.MED.REC. 1398.3.182)

In a double-blind clinical trial study, 128 patients undergoing abdominal surgery were selected and randomly divided into four groups of 32 . In the 4 groups $0.5 \mathrm{Mg} / \mathrm{kg}$ dexmedmotidine, $0.5 \mathrm{mg}$ / $\mathrm{kg}$ pethidine, $0.1 \mathrm{Mg} / \mathrm{kg}$ Ondansetron and the same volume of normal saline were injected intravenously when anesthetics were discontinued and the incidence and severity of postoperative shivering were determined and compared in four groups.

Results: The incidence of postoperative shivering was $12.5 \%$ in the dexmedmotidine group, $31.3 \%$ in the Ondansetron group, 31.3\% in the pethidine group and $50 \%$ in the control group ( $P$ $=0.015)$. The mean of shivering severity in the four groups was $1.33 \pm 0.5,0.17 \pm 0.8,1.09 \pm$ 0.4 and $1.13 \pm 0.39$, and the difference between the four groups was significant $(P=0.005)$.

Discussion: The use of all three drugs of Dexmedetomidine, Ondansetron and pethidine are effective in decreasing the incidence of postoperative shivering, but the use of dexmedetomidine is associated with less postoperative shivering, better hemodynamic stability, and fewer other postoperative complications.

\section{REFERENCES:}

1. Teymourian $\mathrm{H}$, et al. Effect of ondansetron on postoperative shivering after craniotomy. World neurosurgery, 2015, 84.6: 1923-1928.

2. Safavi M, et al. Prophylactic effects of intrathecal Meperidine and intravenous Ondansetron on shivering in patients undergoing lower extremity orthopedic surgery under spinal anesthesia. Journal of research in pharmacy practice, 2014, 3.3: 94.

3. Tahereh P, Dabir S, Badiolzaman R. Efficacy of pethidine and buprenorphine for prevention and treatment of postanesthetic shivering. Tanaffos, 2007, 6.3: 54-58.

4. Milne Richard J,Heel Rennie C. Ondansetron. Drugs, 1991, 41.4: 574-595.

5. Edwards David, et al. Clinical pharmacokinetics of pethidine: 1982. Clinical pharmacokinetics, 1982, 7.5: 421-433.

6. Mather Laurence E, Meffin Peter J. Clinical pharmacokinetics pethidine. Clinical pharmacokinetics, 1978, 3.5: 352-368. 
7. HE, K.; ZHAO, H.; ZHOU, H. C. Efficiency and safety of ondansetron in preventing postanaesthesia shivering. The Annals of the Royal College of Surgeons of England, 2016, 98.6: 358-366.

8. $\mathrm{KH}$ Isazadehfar, et al. The effect of ondansetron in prevention of postoperative shivering after general anesthesia in gynecological surgery. Iranian Red Crescent Medical Journal, 2012, 2012.5, May: 316-317.

9. Solhpour Ali, et al. A comparison of prophylactic use of meperidine, meperidine plus dexamethasone, and ketamine plus midazolam for preventing of shivering during spinal anesthesia: a randomized, double-blind, placebo-controlled study. Journal of clinical anesthesia, 2016, 34: 128-135.

10. Bae Hong-Beom. Dexmedetomidine: an attractive adjunct to anesthesia. Korean Journal of anesthesiology, 2017, 70.4: 375-376.

11. Kim Yong-Shin, et al. Optimal dose of prophylactic dexmedetomidine for preventing postoperative shivering. International journal of medical sciences, 2013, 10.10: 1327.

12. Abdel-Ghaffar, Hala Saad, et al. Safety and efficacy of dexmedetomidine in treating post spinal anesthesia shivering: a randomized clinically controlled dose-finding trial. Pain physician, 2016, 19.4: 243-253.

13. Thomas, Anil, et al. Comparison of different regimens of intravenous dexmedetomidine on duration of subarachnoid block. Journal of anaesthesiology, clinical pharmacology, 2016, 32.4: 497.

14. Doufas Anthony G., et al. Dexmedetomidine and meperidine additively reduce the shivering threshold in humans. Stroke, 2003, 34.5: 1218-1223.

15. NAsseri, Karim; Ghadami Negin, Nouri Bijan. Effects of intrathecal dexmedetomidine on shivering after spinal anesthesia for cesarean section: a double-blind randomized clinical trial. Drug design, development and therapy, 2017, 11: 1107.

16. Bicer, C., et al. Dexmedetomidine and meperidine prevent postanaesthetic shivering. European journal of anaesthesiology, 2006, 23.2: 149-153. 


\section{REGIONAL AND ACUTE PAIN}

\section{A Phase IV Real-World Study on The Use of Low-Dose Methoxyflurane (Penthrox ${ }^{\mathrm{TM}}$ ) for the Treatment of Moderate to Severe Trauma Pain in the Canadian Emergency Department (ADVANCE-ED): An Interim Report on Adverse Events}

David V. Flusk ${ }^{1}$; Samuel Campbell ${ }^{2}$; Loretta Belle Blagrove ${ }^{3}$; Paula S. Piraino ${ }^{3}$; Sonja Dhani ${ }^{3}$

1 Faculty of Medicine, Memorial University of Newfoundland, St. John's NL, Canada

2 Charles V. Keating Emergency and Trauma Centre, QEII Health Sciences Centre, Halifax, NS, Canada

3 Scientific Affairs, Purdue Pharma Canada, Pickering, ON, Canada

Introduction: Inhaled low-dose methoxyflurane (MEOF) is approved in Canada for short-term relief of moderate to severe acute pain associated with trauma or interventional medical procedures in conscious adult patients ${ }^{1}$. The most common adverse reactions are CNS-related and are generally mild and transient. The data from pivotal studies, however, were limited by restricted enrolment criteria, with exclusion of patients with severe $(\geq 7)$ pain, and those using OTC or stably dosed analgesics for other conditions, including chronic pain. ADVANCE-ED is an ongoing phase IV, prospective open-label study undertaken to generate real-world evidence through evaluation of the safety and effectiveness of low-dose MEOF in Canadian Emergency Departments (EDs). The study includes patients with severe pain and those on prior analgesics to evaluate the product efficacy and safety profile in routine clinical practice.

Methods: Ethics approval was obtained from the local REBs of the participating hospitals and all patients (or their legal representatives) provided written informed consent prior to enrolment. This multi-centre study is enrolling adult ( $\geq 18 \mathrm{yrs}$ ) patients with moderate to severe acute pain $\left(\mathrm{NRS}_{0-10} \geq 4\right)$ associated with minor trauma. Eligible patients receive a single treatment of up to $2 \times 3 \mathrm{~mL}$ MEOF (2nd $3 \mathrm{~mL}$ to be provided only upon request), selfadministered by the patient under medical supervision. Rescue medication, if required, is permitted at any time.

Results: Here we describe the safety profile of inhaled MEOF at $50 \%$ enrolment $(n=49)$ as compared with the expected profile based on the pivotal trauma study. Mean (SD) patient age was 48.0 (17.1) yrs, and $55.1 \%$ were female. Limb trauma injuries were most common (87.8\%) with mean $\mathrm{NRS}_{0-10}$ pain (SD) at enrolment of 8.3 (1.5). Routine concomitant medications included lipid modifying agents (20.4\%), antidepressants (18.4\%), GERD medications (12.2\%), beta blocking agents (10.2\%), anxiolytics (8.2\%) and calcium channel blockers $(8.2 \%)$. Medications administered related to the trauma in advance of consent (self-administered or by pre-hospital/hospital) included acetaminophen (40.8\%), NSAIDs (18.4\%) and opioids (16.3\%). Post-consent, NSAIDs (16.3\%), opioids (16.3\%), acetaminophen $(10.2 \%)$ and anesthetics (6.1\%) were used. Adverse events (AEs) were reported by $42.9 \%$ of patients, in line with nearly $50 \%$ in the pivotal trauma study. Dizziness was the most common treatmentrelated $\operatorname{AE}(n=14,28.6 \%)$, consistent with the pivotal study $(29.5 \%)$. Somnolence $(n=2,4.1 \%)$ was reported less frequently than the pivotal trial $(5.4 \%)$, and there were no reports of headache (vs. $21.5 \%$ in the pivotal trial). Euphoric mood $(n=4,8.2 \%)$ was reported more often here than in the pivotal trauma trial. No serious AEs have been reported. One patient $(2.0 \%)$ experienced an $A E$ (nausea) that led to discontinuation of inhaled MEOF. 
Conclusion: Based on $50 \%$ of the patients enrolled in this prospective, open-label study, responses to inhaled low-dose MEOF are within expectation for tolerability.

\section{REFERENCES:}

1. PENTHROX тм Product Monograph, Purdue Pharma (Canada), April 6, 2018. 


\section{Adjuncts to Local Anesthetic Wound Infiltration for Postoperative Analgesia: A} Systematic Review

Dong $\mathrm{An}^{1}$; Johnny-Wei Bai ${ }^{1}$; Anahi Perlas ${ }^{1}$; Vincent W.S. Chan ${ }^{1}$

1 Department of Anesthesiology and Pain Management, Toronto Western Hospital, University Health Network, University of Toronto, Toronto, Canada

Introduction: Local anesthetics are commonly infiltrated into surgical wounds for postoperative analgesia. While many adjuncts to local anesthetic agents have been studied, it is unclear which adjuncts are most effective for co-infiltration to improve and prolong analgesia.

Methods: Ethics approval was not applicable because the study did not involve human or animal research. We performed a systematic review on adjuncts (excluding epinephrine) to local infiltrative anesthesia to determine their analgesic efficacy and opioid-sparing properties. Multiple databases were searched up to December 2019 for randomized controlled trials (RCT) and two reviewers independently performed title/abstract screening and full-text review. Inclusion criteria were 1) adult surgical patients and; 2) adjunct and local anesthetic agents infiltration into the surgical wound or subcutaneous tissue for postoperative analgesia. To focus on wound infiltration, studies on intra-articular, peri-tonsillar, or fascial plane infiltration were excluded. The primary outcome was reduction in postoperative opioid requirement. Secondary outcomes were time-to-first analgesic use, postoperative pain score, and any reported adverse effects.

Results: We screened 6,670 citations, reviewed 126 full-text articles, and included 90 RCTs. Adjuncts included opioids, non-steroidal anti-inflammatory drugs (NSAIDs), steroids, alpha-2 agonists, ketamine, magnesium, neosaxitoxin, and methylene blue. Alpha-2 agonists have the most evidence to support their use as adjuncts to LA infiltration. Fentanyl, NSAIDs (especially ketorolac), dexamethasone, and magnesium show potential as adjuncts but require more evidence. Most studies support the safety of these agents.

Conclusion: Our findings suggest benefits of several adjuncts to local infiltrative anesthesia for postoperative analgesia. Further well-powered RCTs are needed to compare various infiltration regimens and agents. 
Analgesic Efficacy of the Femoral Articular Branch Block in Ambulatory Hip Arthroscopy. A Retrospective Chart Review

Vivesh Patel $^{1}$; Richard Brull ${ }^{1,2,3} ;$ Martino Gabra $^{4}$

1 Department of Anesthesia, Women's College Hospital, Toronto, Canada

2 Department of Anesthesia, University Health Network, Toronto Canada

3 Department of Anesthesia, University of Toronto, Toronto, Canada

4 Department of Pharmacology and Toxicology, University of Toronto, Toronto, Canada

Introduction: Hip arthroscopy is a routinely performed, minimally invasive outpatient procedure that is often associated with a significant amount of postoperative pain. As a result, patients often require high doses of opioids postoperatively. ${ }^{1-4}$ In an effort to reduce pain and opioid consumption, a femoral nerve block (FNB) is often provided in conjunction with multimodal analgesia. A prospective study conducted at Women's College Hospital (WCH) showed that the FNBs were not as effective as desired. As a result, the femoral articular branch (FAB) block replaced the FNB as the standard of care because it theoretically requires less opioids for adequate analgesia in the post-anesthesia care unit (PACU). ${ }^{5-7,12-16}$ This study aims to assess whether the FAB block leads to less opioid consumption and better analgesia among patients that underwent ambulatory hip arthroscopy when compared to patients that received only systemic analgesia or systemic analgesia with a FNB adjunct.

Methods: Ethics approval was obtained from the local REB. The primary sources of data for this retrospective chart review were the hospital medical records for patients that underwent elective hip arthroscopy at WCH during the period of January 2017 to March 2019. Only patients that received systemic analgesia with an adjunct FNB or FAB block or systemic analgesia exclusively were included in this study. An additional source of data for this study was the published retrospective cohort study by Dold ${ }^{6}$ et al and the prospective study by Xing et al. ${ }^{7}$

Results: Post-operative opioid consumption was compared between patients that received a FAB block, a FNB, or systemic analgesia exclusively using a one-way ANOVA test, adjusting for $B M I$, and ASA. Results of a one-way ANOVA were statistically significant, implying a difference between the groups $(P$ value $=0.0002)$. A post hoc Bonferroni's multiple comparisons test showed that there was a statistically significant difference in post-operative opioid consumption between patients that received no block when compared to patients that received the FNB $(P<$ $0.001)$ and when compared to patients that received the FAB block $(P<0.01)$. There was a $P$ value $<0.2$ when comparing postoperative opioid consumption between the FAB block and FNB. All analyses were conducted using SPSS, all P-value were two-sided, and were considered statistically significant if $\mathrm{P}<0.05$.

Discussion: The results showed that there was not a statistically significant difference in postoperative opioid consumption between patients that received the FNB and the FAB block. However, the administration of a block resulted in a statistically significant difference in opioid consumption when compared to receiving systemic analgesia exclusively. There were limitations to this study which may have been better suited for a multivariate analysis. Furthermore, secondary outcomes such as postoperative complications, VAS pain scores, and discharge time should be compared between the groups. 


\section{REFERENCES:}

1. Lee EM, Murphy KP, Ben-David B. Postoperative analgesia for hip arthroscopy: combined L1 and L2 paravertebral blocks. J Clin Anesth. 2008;20(6):462-5.

2. Childs S, Pyne S, Nandra K, Bakhsh W, Mustafa SA, Giordano BD. The Effect of Intraarticular Cocktail Versus Femoral Nerve Block for Patients Undergoing Hip Arthroscopy. Arthrosc - J Arthrosc Relat Surg [Internet]. 2017;33(12):2170-6. Available from: http://dx.doi.org/10.1016/j.arthro.2017.06.036

3. Yu HC, Al-Shehri M, Johnston KD, Endersby R, Baghirzada L. Anesthesia for hip arthroscopy: a narrative review. Can J Anesth. 2016;63(11):1277-90.

4. Bech NH, Haverkamp D, van Leuken LLA, Spuijbroek JA, Hulst AH. Perioperative pain management in hip arthroscopy; what options are there? J Hip Preserv Surg. 2016;3(3):181-9 5. Ward JP, Albert DB, Altman R, Goldstein RY, Cuff G, Youm T. Are femoral nerve blocks effective for early postoperative pain management after hip arthroscopy? Arthrosc - J Arthrosc Relat Surg. 2012;28(8):1064-9.

6. Dold AP, Murnaghan L, Xing J, Abdallah FW, Brull R, Whelan DB. Preoperative femoral nerve block in hip arthroscopic surgery: A retrospective review of 108 consecutive cases. Am J Sports Med. 2014;42:144-9.

7. Xing JG, Abdallah FW, Brull R, Oldfield S, Dold A, Murnaghan ML, et al. Preoperative femoral nerve block for hip arthroscopy: a randomized triple-masked controlled trial. Am J Sports Med. 2015;43(11):2680-7.

8. Birnbaum K, Prescher A, Heßler S, Heller KD. The sensory innervation of the hip joint - An anatomical study. Surg Radiol Anat. 1997;19(6):371-5.

9. Wertheimer L. The sensory nerves of hip joint. J Bone Jt Surg Am. 1952;34-A(2):477-87.

10. Gardner E. The innervation of the hip joint. Anat Rec. 1948;101:353-71.

11. Gerhardt M, Johnson K, Atkinson R, Snow B, Shaw C, Brown A, et al. Characterisation and classification of the neural anatomy in the human hip joint. HIP Int. 2012;22(1):75-81.

12. Girón-Arango L, Peng PWH, Chin KJ, Brull R, Perlas A. Pericapsular Nerve Group (PENG) Block for Hip Fracture. Reg Anesth Pain Med. 2018;43(8):859-63.

13. Ueshima $\mathrm{H}$, Otake $\mathrm{H}$. Clinical experiences of pericapsular nerve group (PENG) block for hip surgery. J Clin Anesth. 2018;51:60-1.

14. Ueshima $\mathrm{H}$, Otake $\mathrm{H}$. Pericapsular nerve group (PENG) block is effective for dislocation of the hip joint. J Clin Anesth. 2019;52:83.

15. Tanzer M, Taddei R, Arbeid E, Zaouter C, Hemmerling TM. Block of the articular branches of the femoral nerve improves early pain control following total hip arthroplasty. Int J Perioper Ultrasound Appl Technol. 2012;1(3):94-8.

16. Yavuz F, Yasar E, Ali Taskaynatan M, Goktepe AS, Tan AK. Nerve block of articular branches of the obturator and femoral nerves for the treatment of hip joint pain. J Back Musculoskelet Rehabil. 2013;26(1):79-83. 


\section{MIXED CATEGORY}

\section{Approach to Epidermolysis Bullosa: When It Could Potentially Be the Last Anesthetic!}

Keerthi Rao ${ }^{1}$; Richa Sharma ${ }^{2}$; Joel Fish ${ }^{3}$; Clyde Matava ${ }^{4}$

1 Clinical Fellow, Pediatric Anesthesia, Department of Anesthesia and Pain management, The Hospital for Sick Children, Toronto, Canada

2 Staff Anesthesiologist, Department of Anesthesia and Pain Management, The Hospital for

Sick Children, Toronto, Canada

3 Associate Professor, Plastic and Reconstructive Surgery, The Hospital for Sick Children, Toronto, Canada

4 Assistant Professor, Department of Anesthesia and Pain management, The Hospital for Sick Children, Toronto, Canada

Introduction: Epidermolysis bullosa (EB) is a rare genetic disorder where excessive skin and mucous membrane fragility lead to bullae formation and ulceration. These patients require multiple procedures in their lifetime and anesthetic challenges encountered include preserving skin integrity, preventing further damage, difficulty in intravenous and airway access owing to scarring and contractures. We describe the novel use of Self-Assembled Skin Substitute (SASS) autologous graft application for the 'curative' management of EB skin lesions. This report highlights a possible long term solution to the dermatologic issues in EB and an appropriately tailored anesthetic technique to aid graft uptake and survivability.

Case presentation: Patient consent was obtained for publication of this case. A 29-year-old male with recessive dystrophic EB was encountered thrice for debridement of the skin lesions on the back, application of the SASS graft and dressing change. Significant preoperative findings were ulceration on anterior and posterior chest, difficult intravenous access, limited neck extension, Mallampatti class IV and $1.5 \mathrm{~cm}$ mouth opening. Anesthesia planning included the surgeons, nursing team and designation of specific roles (IV, airway, sedation) to the members of anesthesia team including a strict "No touch" policy unless required amongst the team members.

During the first anesthetic intravenous access was secured under ultrasound guidance, sedation instituted with midazolam, remifentanil and dexmedetomidine infusions. Upon achieving topical airway anesthesia using lidocaine sprays and nebulization, a 6 cuffed armored endotracheal tube was placed through the right nostril using a $4.5 \mathrm{~F}$ fibreoptic bronchoscope and secured with sutures. Non-adhesive silicone based dressings (IV DermTM, MepitacTM) were used on intravenous access and taping the eyes shut. Head, face and pressure points were well padded prior to positioning prone, monitoring was modified using a clip on saturation probe on the ear, non-invasive blood pressure cuff on a well-padded arm with prolonged cycling interval, and electrocardiogram electrodes on defibrillation gel pad pieces placed on the back.

During the encounter for SASS graft application, the same technique described above was followed, but there were additional concerns to promote graft survivability. Hypotensive episodes were encountered and mean arterial pressure was maintained above $65 \mathrm{mmHg}$ using intravenous phenylephrine boluses to ensure adequate tissue perfusion. 
During the encounter for dressing change, sedation was instituted with sublingual lorazepam sedation and nitrous oxide by mask, thereby preserving hemodynamic stability. Good graft take was noted at this time.

Discussion: The SASS graft could be a potential long term dermal solution in EB and in addition to the routinely practiced modifications and caution, it is vital to promote graft-take by ensuring adequate perioperative tissue perfusion. This encounter also provided us with an opportunity to review the documented literature and attempt to formulate guidelines for the management of such a case for the best possible outcome.

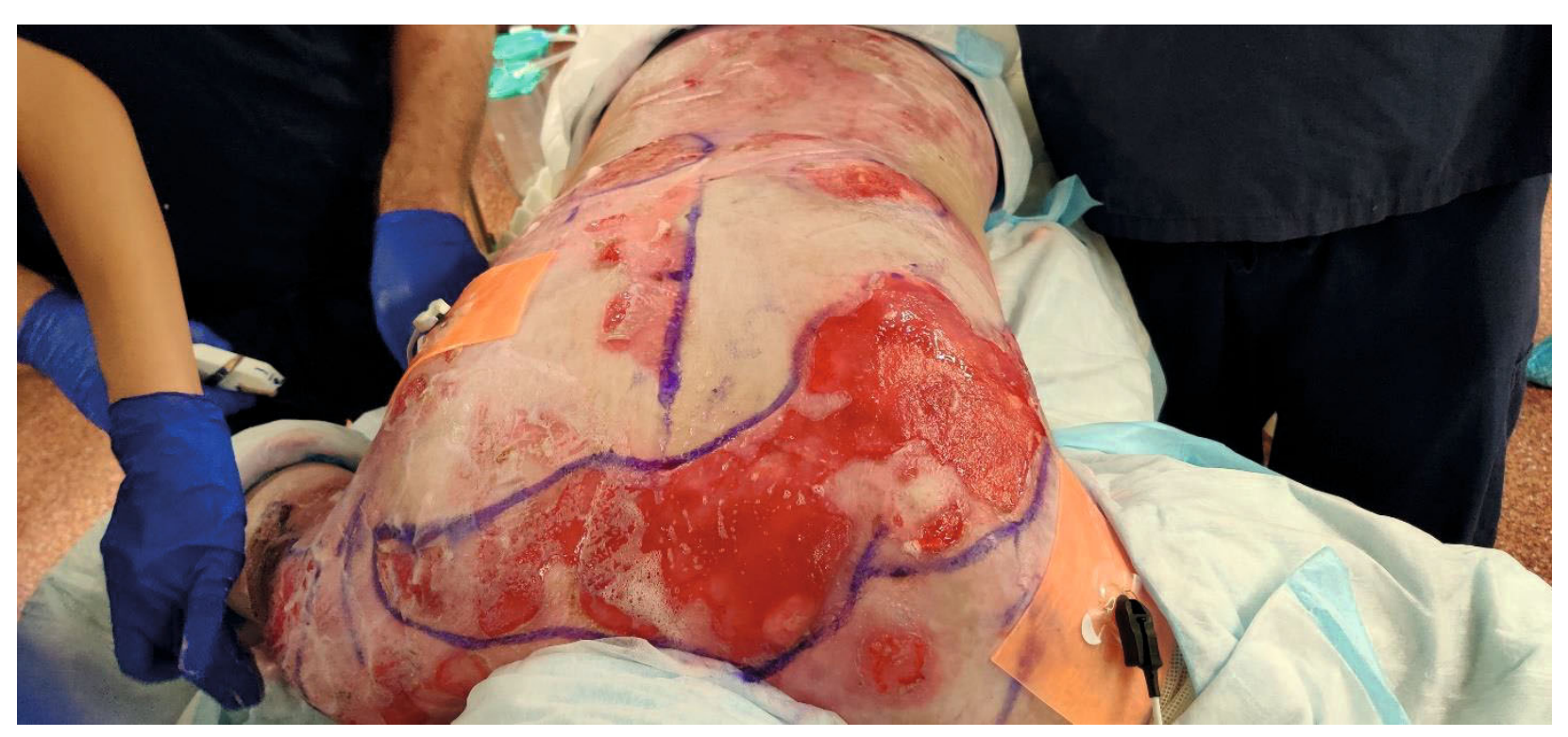




\section{Assessment of Burnout Syndrome Among Anesthesia Providers from a Low Income Country: A Quantitative Analytical Cross Sectional Study}

Eugene Tuyishime ${ }^{1,2,3}$; Paulin Ruhato Banguti ${ }^{1}$; Dylan Bould ${ }^{4}$

1 University of Rwanda, Kigali, Rwanda

2 University Teaching Hospital of Kigali, Kigali, Rwanda

3 OhioHealth Learning, Riverside Methodist Hospital, Columbus, Ohio, USA

4 Children's Hospital of Eastern Ontario, Ottawa, ON, Canada

Introduction: Despite having many studies done in high income countries, there is paucity of data on burnout for anesthesia providers in low-middle income (LMI) countries. Therefore this study aimed 1) to evaluate the prevalence of burnout amongst anesthesia providers in a singular LMI country and 2) to determine factors associated with burnout amongst anesthesia providers in this singular LMI country.

Method: Ethics approval (No 056/CMHS-IRB/2018) was obtained from the local University of Rwanda/College of Medicine and Health Sciences institutional review board.

Design: A quantitative analytical cross sectional study

Setting: Anesthesia providers from 12 hospitals including 4 teaching hospitals, 1 referral hospital, 1 provincial hospital, and 6 district hospitals.

Main outcomes:

1) Mean score response on 22 questions of the full Maslach Burnout Index Health System Survey (MBI-HSS): 9 for emotional exhaustion, 5 assessing depersonalization, and 8 for personal accomplishment.

2) Factors associated with burnout among anesthesia providers.

Results: The response rate was $72.3 \%$ (99/137) with the majority of respondents men (63.6\%), married $(64.3 \%)$, from teaching hospitals $(76.1 \%)$, from urban area $(85.7 \%)$, non physician $(66.7 \%)$, and with experience of below 5 years $(73.7 \%)$. The mean age was $34.6(\mathrm{SD}=6.4)$ and the average weekly working hours was 57.5 hours $(S D=12.1)$. The prevalence of burnout (defined as high emotional exhaustion or high depersonalization) amongst anesthesia providers in this singualr LMI hospitals was $47.5 \%$.

Measured level of burnout as assessed by high emotional exhaustion, low personal accomplishment, and high depersonalization was $46,15.2$ and $8.1 \%$, respectively. Using a multiple logistic regression, we detected that sleeping hours, rights about the schedule, right drugs, right team, and sufficient salary were independently associated with burnout.

Discussion: The prevalence of BOS among anesthesia providers in this singular LMI is high and of major concern. The study identifies key areas upon which interventions could be planned namely adequate sleep, appropriate schedule, right drugs, team support structures, and sufficient remuneration. 


\section{Ketamine Co-Induction for Major Depressive Disorder}

Jordon Steeg ${ }^{1}$; Jonathan Gamble ${ }^{1}$; Jagmeet Bajwa ${ }^{1}$

1 Department of Anesthesiology, Perioperative Medicine and Pain Management, University of Saskatchewan, Saskatoon, Canada

Background: Major depressive disorder (MDD) is common, with a life prevalence of up to $11.3 \%$ amongst Canadians. ${ }^{1,2} \mathrm{~A}$ majority of these patients will require an operation and are at risk of both worsening depressive symptoms and increased postoperative pain. ${ }^{3}$ Ketamine has been demonstrated to rapidly improve MDD symptoms. ${ }^{4,5,6}$ However, the effect of ketamine on MDD symptoms when used as an adjunctive induction agent for general anesthesia remains largely unstudied. ${ }^{7}$

Methods: Ethics approval was obtained from the local REB. This single-center, blinded, randomized clinical trial planned a convenience sample of 50 patients aged 18-65 years with self-reported diagnosis of MDD, currently taking a medication prescribed for MDD, and presenting for gynecologic surgery requiring general anesthesia. Patient and assessor were blinded to group allocation. Enrolled patients were computer-randomized to receive either a general anesthetic which included $0.5 \mathrm{mg} / \mathrm{kg}$ of ketamine on induction (intervention arm) or a general anesthetic absent of ketamine (control arm). MDD severity was scored using the validated Montgomery-Asberg Depression Rating Scale (MADRS) measured preoperatively, upon post-anesthetic care unit (PACU) discharge, and on postoperative days 1, 3, 7, and 30. Other measures included verbally reported pain score (VRS) observed on the same time intervals as well as opiate analgesia requirement in PACU in milligram morphine equivalents. Primary outcome was MADRS on postoperative day 1. Patients were surveyed during their hospital admission when possible, but were reached by telephone after hospital discharge. MADRS and VRS scores were analyzed using repeated measures mixed ANOVA.

Results: 50 women with a mean age of 41 years (SD 11.5) were recruited. The baseline characteristics between groups were similar. Mean and $95 \%$ confidence intervals $(\mathrm{CI})$ for MADRS score on postoperative day 1 were similar with 7.4 (6.1 to 8.8) and 5.6 (4.2 to 7.1) in the control and ketamine groups respectively; VRS scores at $24 \mathrm{~h}$ postoperatively were also similar at 3.7 (3.0 to 4.4) and 3.6 (2.9 to 4.4) in the control and ketamine groups respectively. Opiate analgesic requirements were 6.9 milligrams (SD 6.7) and 7.5 milligrams (SD 7.7) in the control and ketamine groups respectively.

Conclusion: This study suggests that ketamine as a co-induction agent as part of general anesthesia for patients with MDD requiring gynecologic surgery does not reduce the severity of MDD or pain scores on postoperative day 1 . This may be due to a low baseline MDD severity and surgery not normally associated with severe postoperative pain.

\section{REFERENCES:}

1. Bloom D, Cafiero E, Jané-Llopis E, Abrahams-Gessel S, Bloom L, Fathima S, et al. The global economic burden of noncommunicable diseases. Geneva: World Economic Forum; 2011. 
2. Pearson C, Janz T, Ali J. Mental and substance use disorders in Canada [Internet]. Ottawa, ON: Statistics Canada, Health Statistics Division; 2013 Sep 18 [cited 2017 Mar 24]. Available from: http://www.statcan.gc.ca/pub/82-624-x/2013001/article/11855-eng.htm .

3. Ghoneim M, O'Hara M. Depression and postoperative complications: an overview. BMC Surg. 2016;16(5):1-10.

4. Krystal J, Karper L, Seibyl J, Freeman G, Delaney R, Bremmer J, et al. Subanesthetic effects of the noncompetitive NMDA antagonist, ketamine, in humans: psychotomimetic, perceptual, cognitive, and neuroendocrine responses. Arch Gen Psychiatry. 1994;51(3):199-214.

5. Berman RM, Cappiello A, Anand A, Oren DA, Heninger GR, Charney DS, et al. Antidepressant effects of ketamine in depressed patients. Biol Psychiatry. 2000;47(4):3514.

6. Caddy C, Giaroli G, White TP, Shergill SS, Tracy DK. Ketamine as the prototype glutamatergic antidepressant: pharmacodynamic actions, and a systematic review and meta-analysis of efficacy. Ther Adv Psychopharmacol. 2014;4(2):75-99.

7. Kudoh A, Takahira Y, Katagai H, Takazawa T. Small-dose ketamine improves the postoperative state of depressed patients. Anesth Analg. 2002;95(1):114-8. 
Postoperative Pneumonia Prevention: A Retrospective and Cross-Sectional Survey Based Review of How We're Doing

Naveen Sivaranjan ${ }^{1}$; Hamsa Asif ${ }^{1}$; Jessica Pudwell ${ }^{1}$; Romy Nitsch ${ }^{1}$

1 Department of Obstetrics and Gynecology, Kingston General Hospital, Kingston, Canada

Background: Pneumonia is the third most common postoperative complication in surgical patients. The iCOUGH Pulmonary Program, developed by Boston University School of Medicine, employed 6 simple intervention strategies including incentive spirometry, coughing and deep breathing, oral care, patient education, early mobilization, and head of bed elevation, that dropped the incidence of postoperative pneumonia from $2.6 \%$ to $1.6 \%$ at their center. Our project utilizes a retrospective chart review and a cross-sectional survey of multidisciplinary care providers examining the current state of the frequency of these interventions to prevent postoperative pneumonia at Kingston General Hospital (KGH).

Methods: Patients were identified through medical records diagnosis codes and charts were reviewed to identify the incidence of postoperative pneumonia as well as the utilization of iCOUGH related interventions. A cross-sectional survey of care providers working on postoperative wards in General Surgery, Orthopedics, and Obstetrics/Gynecology was conducted. The survey focused on the care providers' understanding of the incidence of postoperative pneumonia in relation to other wards at $\mathrm{KGH}$ and to other hospitals in our area, their personal application of postoperative pneumonia prevention techniques, and the major barriers to intervention implementation.

Results: Of the 2420 orthopedic surgery patients, 40 were diagnosed with a postprocedural respiratory disorder during admission. Of these 40 , postoperative pneumonia was found in 27 $(1.12 \%)$ of cases. A total of 4 patients sat up in their chair and 1 walked on postoperative day (POD) 0. Patients were up in their chairs $3 x /$ day by POD $4.29 \pm 4.44$ and ambulating $3 x /$ day by POD $6.3 \pm 4.32$. A total of 55 care providers were surveyed. Most care providers did not know the incidence of pneumonia relative to other hospitals (33/55) and other floors (29/55) at KGH. All respondents could identify at least 1 intervention to prevent pneumonia (mean $2.87 \pm 1.05$ ), however, no one could list more than 5. "Lack of patient engagement" (29/55) and "inadequate staffing" (23/55) were noted as the major barriers to implementing interventions, with feedback suggesting that patient education should be a target for improvement.

Discussion: Poor patient awareness, as well as lack of patient engagement in interventions, are major barriers that prevent healthcare providers from performing the interventions to prevent postoperative pneumonia. From our cross-sectional survey, it's clear that the next focus should be improving staff and patient education regarding postoperative pneumonia.

\section{REFERENCES:}

Cassidy, Michael R., et al. I COUGH: reducing postoperative pulmonary complications with a multidisciplinary patient care program. JAMA surgery 2013;148(8): 740-745.

Smetana GW. Postoperative pulmonary complications: an update on risk assessment and 
reduction. Cleve Clin J Med. 2009;76(suppl4):S60-S65

\section{Sugammadex Use Audit in the Main Operating Room (OR) of a Large, Tertiary Care Hospital}

Christopher L Pysyk ${ }^{1}$

1 Department of Anesthesiology and Pain Medicine, The Ottawa Hospital, University of Ottawa, Ottawa, ON, Canada

Introduction: Sugammadex became available for clinical use in 2017 at The Ottawa Hospital $(\mathrm{TOH})$, a tertiary care, academic health sciences centre (AHSC). Indications for sugammadex use at $\mathrm{TOH}$ included reversal of rocuronium-induced neuromuscular blockade when reversal was not possible or incomplete with neostigmine. To better understand the number and context of sugammadex administrations, the Quality \& Patient Safety (QPS) Committee of the Department of Anesthesiology and Pain Medicine conducted an audit of Main OR sugammadex utilization.

Methods: Ethics approval was waived by the local REB. Using data available in the electronic Anesthesia Information Management System at TOH, a list of all patients administered sugammadex in the first 24 months of sugammadex availability was provided to the QPS Committee. From this list, a random number-generated, convenience sample of $10 \%$ of patients who had received sugammadex was reviewed. The audit assessed: type and urgency of surgery, age and sex of patient, presence of neuromuscular blockade monitoring, timing and dose of muscle relaxant and sugammadex administered, and presence of trainee. A monthly report of total sugammadex administrations in the Main OR was also generated.

Results: Sugammadex administration events increased for each successive 6-month interval over the first 24 months of sugammadex availability. 42 of $418(10 \%)$ cases with sugammadex administration were reviewed. Male patients represented 25 of 42 cases (59\%). Non-elective patients accounted for 19 of 42 cases (45\%). 18 of 42 cases (43\%) involved sugammadex administration after $1530 \mathrm{~h}$. Residents provided care in 20 of 42 cases (48\%) where sugammadex was administered. General surgery accounted for the largest surgical patient population (19 of 42 cases, $45 \%$ ) receiving sugammadex. No cases of sugammadex use for emergency airway rescue were noted. 29 of 42 cases (69\%) met institutional indications for sugammadex administration. Neuromuscular transmission (NMT) monitoring was documented in 37 of 42 cases (88\%). Attributable use of sugammadex was related to practice management $(19 / 42,45 \%)$ with 5 of $42(12 \%)$ cases involving 0.15 to $0.25 \mathrm{mg} / \mathrm{kg}$ of rocuronium administered within last 30 minutes of the procedure. NMT monitor equipment failure was noted in 8 of 37 $(19 \%)$ cases where NMT monitors were used. Sugammadex was given instead of neostigmine in 5 of 32 cases (16\%) where reversal with neostigmine was possible based on NMT Train of Four values.

Conclusion: The number of sugammadex administrations increased successively in a nonrandom fashion over the first 24 months that sugammadex was made available in the Main OR of a tertiary care AHSC. Though the majority of sugammadex administrations met institutional indications, opportunities for improved stewardship may involve targeted approaches to 
processes of care, such as clinical practice management of rocuronium-induced muscle paralysis, and structural factors, such as ensuring the availability of functional NMT monitors.

Number of Sugammadex administrations in Surgical Information Management System (SIMS) by Month at $\mathrm{TOH}$

80

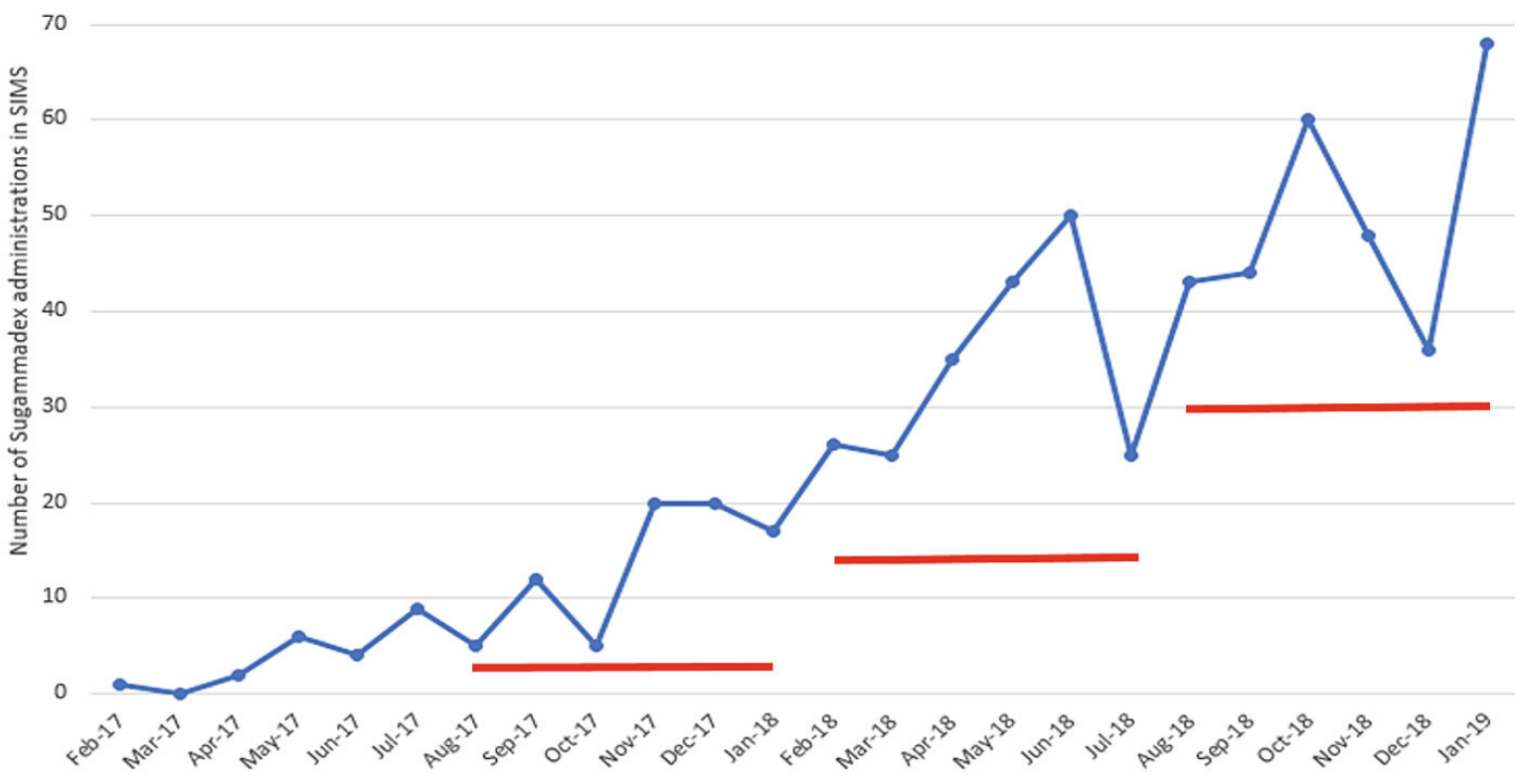

Figure 1 - Run chart displaying the number of sugammadex administrations in Main Operating Room over the first 2 years of sugammadex availability. Red bars correspond to the median number of sugammadex administrations in the preceding 6-month interval. For example, the red bar over August 2017 to January 2018 is the median number of sugammadex administrations from February 2017 to July 2017. 


\title{
RESIDENTS' COMPETITION
}

\author{
BNP as a Screening Tool for Myocardial Infarction and Myocardial Injury after Noncardiac \\ Surgery \\ Varun Suresh $^{1}$; Michael Taylor ${ }^{1} ;$ Albert Tsui $^{2}$; Derek Dillane ${ }^{1}$ \\ 1 Department of Anesthesia, University of Alberta, Edmonton, Canada \\ 2 Department of Laboratory Medicine, University of Alberta, Edmonton, Canada
}

Background: Major cardiac complications are responsible for at least a third of perioperative deaths and are associated with significant morbidity (1-3). Canadian Cardiovascular Society (CCS) Guidelines on Perioperative Cardiac Risk Assessment identify B-type natriuretic peptide $(B N P) \geq 92 \mathrm{ng} / \mathrm{L}$ as an independent predictor of myocardial infarction (MI) up to 30 days after surgery (4). CCS guidelines recommend screening with preoperative BNP and measuring daily high-sensitivity troponin up to 72 hours after surgery when BNP $\geq 92 \mathrm{ng} / \mathrm{L}$. Without cardiac biomarker screening, more than half of all perioperative myocardial infarctions go undetected (4) (5). This silent ischemia is termed myocardial injury after non-cardiac surgery (MINS) (5). It is defined as troponin $T \geq 0.03 \mathrm{ng} / \mathrm{ml}$ (4). MINS has been associated with significantly increased 30-day mortality (6).

\section{Objectives:}

1. Compare the rates of myocardial infarction in the first 30 days after surgery in BNP positive and negative patients

2. Determine the incidence of MINS in the first 72 hours after surgery in BNP positive patients

3. Explore the current management strategy of MINS

Methods: Ethics approval was obtained from the local REB and the study was registered at clinicaltrials.gov (NCT04077294). Patients undergoing elective, noncardiac surgery with an overnight stay were assessed at the Preadmission Clinic. BNP screening was performed in patients who qualified according to CCS guidelines (4). Patients with positive BNP underwent postoperative cardiac monitoring with daily high-sensitivity troponin I (hsTnl). All patients were contacted by telephone at 30 days after surgery to determine incidence of myocardial infarction. Medical records were reviewed if patients had an MI or MINS. Fischer's exact test was used to compare the postoperative incidence of MI between BNP positive and negative patients.

Results: 1348 elective surgical patients were screened in the preadmission clinic between May 21st and September 12th, 2019. 287 patients (21.3\%) qualified for BNP measurement. 70/287 (24.3\%) patients had positive BNP. The incidence of MI within 30 days for BNP positive patients was $2.2 \%(1 / 70)$ and in BNP negative patients was $0.5 \%(1 / 217)$. This was not statistically significant $(p=0.429) .25$ patients were excluded because low sensitivity troponin was measured postoperatively leaving 262 patients for analysis. 13.3\% (6/45) of BNP positive patients had MINS within 72 hours of surgery. None of the MINS patients had an MI or died within 30 days of surgery.

Conclusion: Preoperative BNP screening of at-risk patients undergoing noncardiac surgery was not found to be a valuable tool for predicting patients at risk of postoperative Ml. Even 
though preoperative BNP screening was useful for detecting patients at risk of MINS, we found no evidence of increased cardiac morbidity or mortality in this population.

\section{REFERENCES:}

1. Botto F, Alonso-Coello P, Chan MT, Villar JC, Xavier D, Srinathan S, et al. Myocardial injury after noncardiac surgery: a large, international, prospective cohort study establishing diagnostic criteria, characteristics, predictors, and 30-day outcomes. Anesthesiology. 2014;120(3):564-78

2. Udeh BL, Dalton JE, Hata JS, Udeh CL, Sessler DI. Economic trends from 2003 to 2010 for perioperative myocardial infraction: a retrospective, cohort study. Anesthesiology. 2014;121 (1):36-45

3. Van Waes JA, Nathoe HM, de Graaff JC, Kemperman H, de Borst GJ, Peelen LM, et al. Myocardial injury after noncardiac surgery and its association with short-term mortality. Circulation. 2013;127(23);2264-71

4. Duceppe E, Parlow J, MacDonald P, Lyons K, McMullen M, Srinathan S, et al. Canadian Cardiovascular Society Guidelines on Perioperative Cardiac Risk Assessment and Management for Patients Who Undergo Noncardiac Surgery. Can J Cardiol. 2017;33(1):1732.

5. Devereaux PJ, Xavier D, Pogue J, et al. Characteristics and short-term prognosis of perioperative myocardial infarction in patients undergoing noncardiac surgery: a cohort study. Ann Intern Med 2011; 154:523-8.

6. Bicard B et al. Myocardial Injury After Noncardiac Surgery (MINS) in Vascular Surgical Patients. Annals Surg. 268(2) 


\section{Derivation and External Validation of A 30-Day Mortality Risk Prediction Model for Older Patients Having Emergency General Surgery}

Simon Feng ${ }^{1}$; Carl Van Walraven ${ }^{2,3,4}$; Manoj M. Lalu ${ }^{1,3}$; Husein Moloo ${ }^{3,5}$; Reilly Musselman; Daniel I. Mclsaac ${ }^{1,2,3}$

1 University of Ottawa, Department of Anesthesiology and Pain Medicine, Ottawa, Canada 2 ICES, Toronto, Canada

3 Ottawa Hospital Research Institute, Ottawa, Canada

4 University of Ottawa, Department of Medicine, Ottawa, Canada

5 Department of Surgery, University of Ottawa and The Ottawa Hospital, Ottawa, Canada

Introduction: People $\geq 65$ years old are over-represented among patients who require emergency general surgery (EGS) $)^{1,2}$. These high-risk patients are often medically complex and near the end of life, ${ }^{2}$ creating prognostic and decisional uncertainty. Accurate risk prediction models can support informed consent and ensure clinical decisions align with goals of care. However, current preoperative risk prediction models for older EGS patients have major limitations, and do not address the specific risk profile of older patients ${ }^{3-6}$. Accurate and externally validated models specific to older patients are needed to inform care and decision making. The objective of this study is to derive, internally and externally validate a multivariable model to predict 30 -day mortality in EGS patients $\geq 65$ years old.

Methods: Ethics approval to use National Surgical Quality Improvement Program (NSQIP) data was obtained from the local REB. External validation will use routinely collected anonymized data that are legally exempt from research ethics review. This retrospective cohort study included 50,221 patients from the NSQIP database having 1 of 7 core EGS procedures (appendectomy, cholecystectomy, laparotomy, lysis of adhesions, large and small bowel resection, and peptic ulcer repairs). Predictor variables were pre-specified based on clinical and epidemiological knowledge. Outcome was 30 -day all-cause mortality from the index surgical procedure. The model was derived using logistic regression penalized with elastic net regularization, as well as a machine learning technique called ensemble modelling to aggregate results across 5000 bootstrap samples and reduce overfitting. The model was internally validated with $\mathrm{k}$-fold validation $(\mathrm{k}=10)$ and bootstrap internal validation. Secondary analysis was done including pre-specified lab variables with complete case elastic net regularization analysis. Multiple imputation analysis was done for missing variables. External validation is being conducted using a provincial health database.

Results: Of the 50,221 patients $\geq 65$ years old having EGS procedure between 2012-2016, $6,218(12.4 \%)$ died. Factors associated with mortality include older age, frailty and related characteristics, higher risk surgery, and comorbidities. After tuning of our elastic net logistic regression model, we achieved strong discrimination (area under the curve [AUC] 0.871) and calibration (agreement between observed and predicted risks across the spectrum using Loesssmoothed calibration plots, Figure 1). Internal validation achieved a range of AUC consistent with derivation (K-fold AUC 0.850-0.885, Bootstrap AUC 0.870) with similar Loess-smoothed calibration plots. Addition of lab-based predictors (AUC 0.871) did not improve model discrimination or calibration. 
Conclusion: Derivation and internal validation of a multivariable mortality risk prediction model specific to older people having EGS demonstrated strong discrimination and calibration. A planned external validation is currently underway. Following external validation, clinical testing will be required to evaluate whether this model can support improved decision making for high risk older patients having emergency general surgery.

\section{REFERENCES:}

1. JAMA Surg. 2016;151(6):e160480.

2. Anesth Analg. 2017;124(5):1653-1661.

3. PLoS One. 2016;11(2):e0148820.

4. BMC Med Res Methodol. 2012;12(1):82.

5. Br J Anaesth. 2015;115(6):849-860.

6. Trauma Surg Acute Care Open. 2018;3(1):e000160.

Figure 1 - Loess smoothed calibration plot of observed vs. expected risk of 30-day mortality

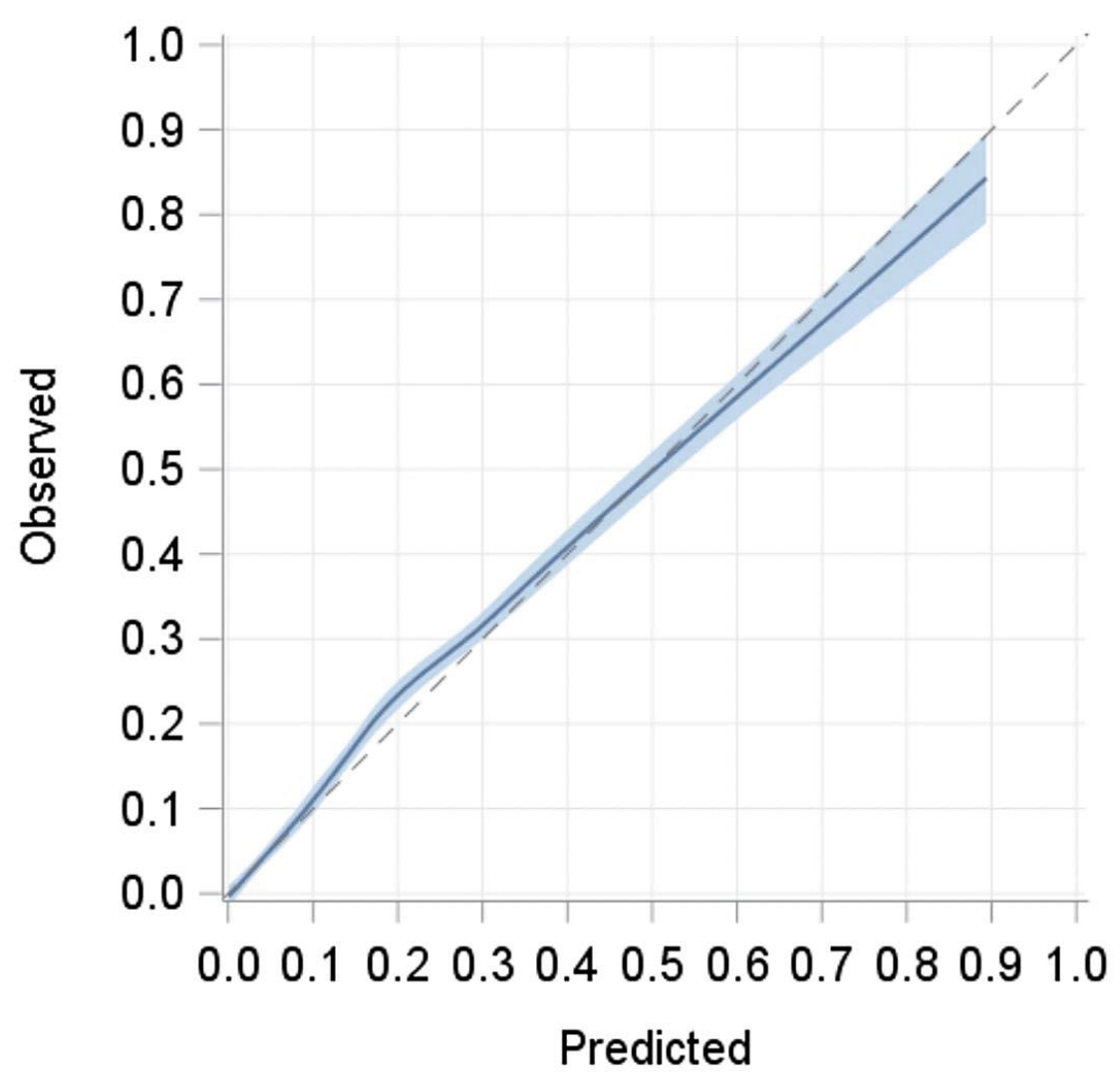

The Loess smoothed calibration plot for the logistic regression model with elastic net regularization shows strong calibration across the entire spectrum of observed versus predicted risk of 30-day mortality. 


\section{Dose-Dependent Effects of Protamine on Coagulation and Platelet Function in The} Context of In Vitro Heparin Reversal

$\underline{\text { Loretta TS Ho }}^{1}$; Gerhardus JAJM Kuiper ${ }^{2}$; Mark McVey ${ }^{1,3}$; Keyvan Karkouti ${ }^{1,2}$

1 Department of Anesthesiology and Pain Medicine, University of Toronto, Toronto, Canada

2 Department of Anesthesiology and Pain Medicine, Toronto General Hospital, Toronto, Canada

3 Department of Anesthesiology and Pain Medicine, SickKids, Toronto, Canada

Introduction: Protamine is the agent of choice for reversal of unfractionated heparin (UFH) in the context of cardiopulmonary bypass (CPB). The current practice consensus of protamine-toheparin-dosing-ratio $(\mathrm{P}: \mathrm{H})$ for adequate reversal is $1: 1$ [1]. Increasingly, studies highlight the paradoxical bleeding risk from excess protamine, favouring a lower $\mathrm{P}: \mathrm{H}$ [2]. At concentrations suitable for CPB, UFH (4 international units $(\mathrm{IU}) / \mathrm{mL}$ ) is known to inhibit coagulation and thrombin generation [3]. Contrarily, protamine's anti-platelet and anti-coagulation effects have not been fully characterized at clinically relevant concentrations.

We evaluated the in-vitro impact of clinically relevant concentrations of protamine with $4 \mathrm{IU} / \mathrm{mL}$ UFH in whole blood $(\mathrm{P}: \mathrm{H}=0.5: 1 ; 1: 1$ and $1.5: 1)$ on platelet function, intrinsic pathway coagulation, and thrombin generation.

Methods: Ethics approval was obtained from the local REB. Protamine $(0,20 \mathrm{ug} / \mathrm{ml}, 40 \mathrm{ug} / \mathrm{ml}$, $60 \mathrm{ug} / \mathrm{ml})$ and $\mathrm{UFH}(0,4 \mathrm{IU} / \mathrm{mL})$ in $\mathrm{P}: \mathrm{H}$ of $0: 1 ; 0.5: 1 ; 1: 1 ; 1.5: 1$ and 1.5:0 were added to venous blood collected from consented healthy volunteers $(n=10)$. Post-incubation (5 minutes, at room temperature) samples were assayed for global measures of intrinsic coagulation in clotting times (CTs) with Thromboelastometry (ROTEM-INTEM and HEPTEM), platelet function (Plateletworks) and thrombin generation (Calibrated Automated Thrombography, CAT). Paired t-tests were applied to detect statistical significance between variable groups from baseline (no heparin or protamine).

Results: Higher P:H of $1: 1$ and 1.5:1 showed significant prolongation of CTs compared to baseline CTs (250 secs \pm 19 and $275 \pm 49$ vs $189 \pm 20 ; p=0.016$ and 0.0001 respectively), while $\mathrm{P}: \mathrm{H}$ of $0.5: 1$ showed no significant change in CTs. Heparin neutralisation using P:H of 1:1 and 1.5:1 significantly impaired recovery of thrombin generation to baseline, as measured by endogenous thrombin potential $\left(E T P=1059 n M^{*} \min \pm 22.3\right.$ and $1214 \pm 38.1$ vs $1406 \pm 44.0 ; p=$ 0.013 and 0.001 ) and peak thrombin generation (Peak=118 $\mathrm{nM} \pm 3.3$ and $143 \pm 3.8$ vs $247 \pm$ $8.1 ; p=0.001$ and 0.004$)$ when compared with low $\mathrm{P}: \mathrm{H}$ of $0.5: 1$. No appreciable platelet dysfunction was detected in all experimental groups after collagen mediated platelet activation using Plateletworks.

Conclusion: Our study shows that in vitro doses of protamine considered to be within current standards of care may lead to coagulation impairment. The clinical relevance of these findings needs to be explored. 


\section{REFERENCES:}

1. Ferraris V, Shore-lesserson L, Baker RA et al. STS / SCA / AmSECT Clinical Practice Guidelines : Anticoagulation During Cardiopulmonary Bypass. 2018: 1-14.

2. Boer C, Meesters MI, Veerhoek D, Vonk ABA. Anticoagulant and side-effects of protamine in cardiac surgery. British Journal of Anaesthesia 2018: 1-14.

3. Griffin MJ, Rinder HM, Smith BR et al. The effects of heparin, protamine, and heparin/protamine reversal on platelet function under conditions of arterial shear stress. Anesthesia and analgesia 2001; 93: 20-7. 


\title{
Glycemic Control in Diabetic Patients Undergoing Elective Surgery: A Feasibility Study Evaluating Perioperative Subcutaneous Basal Bolus Insulin Therapy
}

\author{
Akua Gyambibi ${ }^{1} ;$ Tammy McNab²; Karen Buro; Derek Dillane ${ }^{1}$ \\ 1 Department of Anesthesia and Pain Medicine, University of Alberta \\ 2 Division of Endocrinology and Metabolism, Department of Medicine, University of Alberta \\ 3 Department of Mathematics and Statistics, MacEwan University
}

Introduction: Guidelines recommend blood glucose $(B G)$ values of $5.0-10.0 \mathrm{mmol} / \mathrm{L}$ for diabetics undergoing non-cardiac surgery without defining an optimal insulin strategy (1). Although variable-rate insulin infusions (VRI) are the perioperative standard, subcutaneous basal-bolus insulin therapy (BBIT) is associated with more cost-effective and efficacious glycemic control in non-ICU patients, and has been recommended for intraoperative use in a recently published review $(3,4)$. To date, no studies have examined the use of BBIT in the intra-operative and post-anesthetic care setting (PACU).

Objectives: To determine whether BBIT is a feasible alternative to insulin infusions in surgical patients by comparing 1) perioperative BG values and 2) frequency of hyperglycemic (BG >10 $\mathrm{mmol} / \mathrm{L})$ and hypoglycemic (BG $<5 \mathrm{mmol} / \mathrm{L})$ events.

Methods: Ethics approval was obtained from the local REB for this non-randomized, prospective study (Pro 00077714). Patients who met eligibility criteria (insulin-dependent diabetes (Type 1 and 2), elective surgery < 4 hours duration and $\mathrm{Hgb}$ A $1 \mathrm{C}<10 \%$ within 90 days before surgery) were consented, and patients with significant hepatic disease, renal failure with GFR $<30$, expected large fluid shifts during surgery, or post-operative ICU admission were excluded. Staged recruitment for the BBIT group was followed by that of the VRI group over 8 months. BBIT participants reduced their last dose of basal insulin before surgery and received rapid-acting subcutaneous insulin intra-operatively according to a previously published regimen (4). Capillary BG was measured pre-operatively and every 1-2 hours until discharge from the PACU per protocol. VRI participants received our institution's standard intravenous insulin protocol and monitoring. A two-sample t-test and Fisher exact test were used to analyze the collected data.

Results: Twenty BBIT and 21 VRI patients were recruited. Two outliers with significant hyperglycemia (one in each group) were excluded from further analysis. Pre-operative, intraoperative and PACU mean BG (mmol/L +/- 95\% Cl) in the BBIT group were $8.3+/-1.07$ (SD 2.38), $7.6+/-1.17$ (SD 2.60), and 7.9+/-1.04 (SD 2.12), respectively. Analogous BG values in the VRI group were $8.4+/-1.28$ (SD 2.91), $8.3+/-1.89$ (SD 3.62), and 9.4 +/- 1.35 (SD 3.09). Two-sample t-testing found no difference in mean BG between the BBIT and VRI groups pre-operatively $(p=0.954)$, intra-operatively $(p=0.507)$, and post-operatively $(p 0.102)$. Similarly, a Fisher exact test showed no difference in the number of patients in either group with hyperglycemia or hypoglycemia pre-operatively $(p=0.237)$, intra-operatively $(p=0.173)$, or post-operatively $(p=0.195)$. However, a trend was observed toward lower mean BG with less variation in the BBIT group (Figure 1).

Conclusion: No difference was found between BBIT and insulin infusion groups for the primary and secondary outcomes of BG values and frequency of hyper- or hypoglycemic events. However, trends toward lower glucose values in the BBIT group should be explored in a future study. 


\section{REFERENCES:}

1. Diabetes Canada Clinical Practice Guidelines; In-hospital Management of Diabetes. Can J Diabetes 42 (2018) S115-S123.

2. Moghissi ES, Korytkowski MT, DiNardo M, et al. American Association of Clinical Endocrinologists and American Diabetes Association consensus statement on inpatient glycemic control. Endocr Pract 2009;15:353e69.

3. Umpierrez GE, Smiley D, Zisman A, et al. Randomized study of basal-bolus insulin therapy in the inpatient management of patients with type 2 diabetes (RABBIT 2 trial). Diabetes Care 2007;30:2181e6.

4. Duggan EW, Carlson K, Umpierrez GE. Perioperative Hyperglycemia Management. Anesthesiology. 2017. Mar; 126(3):547-560.
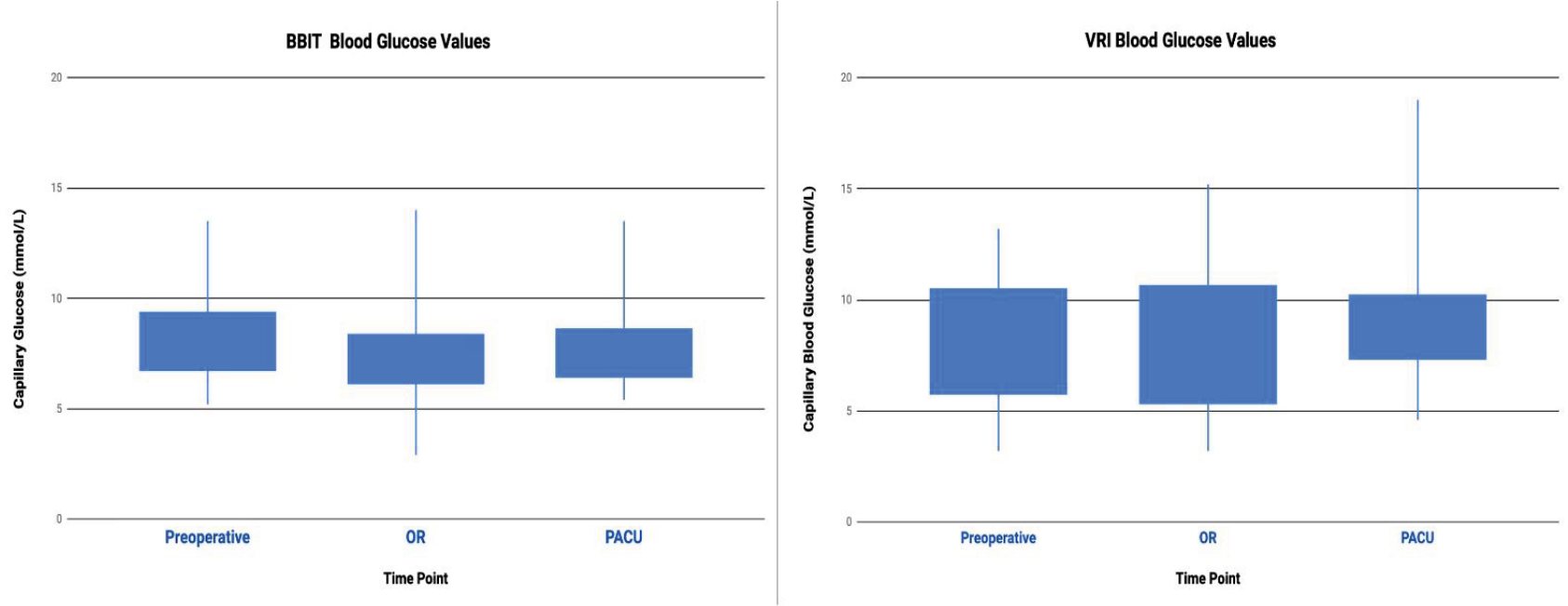

Figure 1. Box-plot comparing blood glucose values in the pre-operative, intra-operative (OR) and post-anesthetic care unit (PACU) period for BBIT (left) and VRI (right) groups. Lower and upper box boundaries represent the 25 th and 75 th percentiles, respectively, and lower and upper error lines represent the maximum and minimum glucose values. 


\section{Low-Dose Ketamine as an Adjunct to Electroconvulsive Therapy Does Not Improve} Psychiatric Outcomes: A Randomized Controlled Trial

Adrianna Woolsey ${ }^{1}$; Jalal Nanji ${ }^{1}$; Chantal Moreau²; Sudhakar Sivapalan²; Stephane Bourque; Alfonso Ceccherini-Nelli"\#; Ferrante Gragasin ${ }^{1 \#}$

1 Department of Anesthesiology and Pain Medicine, University of Alberta, Edmonton, Alberta, Canada

2 Department of Psychiatry, University of Alberta, Edmonton, Alberta, Canada \# denotes equal contribution

Introduction: Electroconvulsive therapy (ECT) is a well-established therapy for Major Depressive Disorder (MDD). Literature suggests that ketamine at low doses may be an alternative therapy for treatment-resistant MDD1. We hypothesized that the addition of low-dose ketamine to anesthesia for ECT would improve depression scores in patients diagnosed with MDD.

Methods: Ethics approval was obtained from the local REB. Patient consent was acquired and off-label use of ketamine was approved by Health Canada for this randomized, double-blinded, placebo-controlled study. Primary outcome was the number of treatments required to achieve a $50 \%$ reduction in the Montgomery-Asberg Depression Rating Scale (MADRS). Secondary outcomes included the number of treatments required to achieve a $25 \%$ reduction in MADRS, and differences in Clinical Global Impression Scale for Severity (CGI-S), mean arterial pressure (MAP), heart rate (HR), and seizure duration. Sample size calculation revealed 14 patients per group was required with $80 \%$ power and alpha $=0.05$. The ketamine dose was increased to 0.5 $\mathrm{mg} / \mathrm{kg}$ IV (from $0.2 \mathrm{mg} / \mathrm{kg}$ ) based on the results of a pre-planned interim analysis after the first 14 recruits. Patients received the study drug prior to propofol induction for each ECT treatment, up to a maximum of 12 treatments.

Results: A total of 45 patients completed the study. There was no difference in the number of ECT treatments required for a $50 \%$ reduction of MADRS between ketamine $(n=16)$ and placebo $(n=15)(8.25 \pm 2.72$ vs. $7.73 \pm 2.89 ; p=0.56)$. There was no difference in the number of ECT treatments required for a $25 \%$ reduction of MADRS ( $4.25 \pm 1.52$ vs. $5.47 \pm 2.94$; p=0.34); CGI-S ( $3.75 \pm 2.99$ vs. $4.73 \pm 2.29 ; p=0.26)$; seizure duration (35.74 \pm 11.82 vs. $35.09 \pm 6.97$ sec; $\mathrm{p}=0.85)$; and peak MAP or peak HR (\% above baseline) $(125.5 \pm 10.2$ vs. 122.7 vs. 9.7; $p=0.44$, and $115.9 \pm 14.6$ vs. $111.8 \pm 13.7 ; p=0.42$, respectively). There was a trend towards decreased propofol dose required in the experimental group (1.13 $\pm 0.31 \mathrm{vs} .1 .36 \pm 0.39 \mathrm{mg} / \mathrm{kg} ; \mathrm{p}=0.08)$. No adverse events were reported.

Discussion: Our results suggest the adjunctive use of ketamine does not improve psychiatric outcomes following ECT. Similar hemodynamic profiles and absence of adverse events suggest that low-dose ketamine may be safely used in this setting; however, even in the presence of potentially reduced propofol dosing in the treatment group, there was no increase in seizure duration. Therefore, indications for use of ketamine in ECT should be limited to those that are patient-specific and not for the goal of therapeutic benefit. While ketamine in isolation may be a useful therapy for MDD, the possibility of a therapeutic "ceiling effect" with ECT may explain why the addition of ketamine did not improve therapeutic outcomes in our study. 


\section{REFERENCES:}

1. Newport DJ, Carpenter LL, McDonald WM, et al. Ketamine and Other NMDA Antagonists:

Early Clinical Trials and Possible Mechanisms in Depression. Am J Psychiatry 2015;172(10):950-966. 
The Accuracy and Feasibility of Clinically Applied Frailty Instruments Before Surgery: A Systematic Review and Meta-Analysis

Sylvie Aucoin ${ }^{1,2,4}$; Mike Hao ${ }^{2}$; Raman Sohi²; Julia Shaw ${ }^{3}$, Itay Bentov ${ }^{5}$; David Walker ${ }^{1}$; Daniel Mclsaac $2,3,4,6$

1 Centre for Perioperative Medicine, University College London, London, United Kingdom

2 Department of Anesthesiology \& Pain Medicine, University of Ottawa, Ottawa, Canada

3 School of Epidemiology \& Public Health, University of Ottawa, Ottawa, Canada

4 Department of Anesthesiology \& Pain Medicine, The Ottawa Hospital, Ottawa, Canada

5 Department of Anesthesiology and Pain Medicine, Harborview Medical Center, Seattle, United

States

6 Clinical Epidemiology Program, The Ottawa Hospital Research Institute, Ottawa, Canada

Introduction: A barrier to routine preoperative frailty assessment is the large number of frailty instruments described. Previous systematic reviews estimate the association of frailty with outcomes, but none has evaluated outcomes at the individual instrument level or specific to clinical assessment of frailty, which must combine accuracy with feasibility to support clinical practice. Lack of clear data on which instrument to use is a recognized barrier to uptake of international guideline-recommended preoperative frailty assessment for all older patients. Therefore, our objective was to systematically review prospective preoperative clinical frailty assessment to determine the instrument-level feasibility and association with high priority outcomes.

Methods: Ethics approval was not applicable because the study did not involve human or animal research. We conducted a pre-registered systematic review (CRD42019107551) of studies prospectively applying a frailty instrument in a clinical setting prior to surgery. Medline, EMBASE, CINAHL and Cochrane databases were searched using a peer-reviewed strategy. All stages of the review were completed in duplicate. The primary outcome was mortality, secondary outcomes included complications, discharge disposition, delirium, length of stay and functional recovery. Effect estimates were pooled using random-effects models. Risk of bias was assessed. Feasibility measures were collected and qualitatively synthesized using directed content analysis.

Results: Seventy studies were included; 45 contributed to meta-analyses. Frailty was defined using 35 different instruments; five instruments had data from at least 3 studies, allowing metaanalysis. The Fried Phenotype was most often studied. Most strongly associated with: mortality and non-favourable discharge was the Clinical Frailty Scale (OR 4.89, 95\% Cl 1.83-13.05 and OR 6.31, 95\% Cl 4.00-9.94, respectively); complications the Edmonton Frail Scale (OR 2.93, $95 \% \mathrm{Cl} 1.52-5.65$ ); and delirium the Frailty Phenotype (OR 3.79, 95\% Cl 1.75-8.22). Thirty-two studies reported aspects of feasibility, and the Clinical Frailty Scale, Edmonton Frail Scale, Frailty Index, and Frailty Phenotype had the most data. The Clinical Frailty Scale had the highest reported measures of feasibility, specifically reported to be fast and easy to use with minimal logistical or environmental barriers. All available data positively supported the Clinical Frailty Scale, the Edmonton Frail Scale and Frailty Index had predominantly positive ratings, however majority of the data for the Frailty Phenotype did not support feasibility. 


\section{Conclusion:}

Preoperative frailty assessment is a guideline recommended aspect of optimal preoperative care for older people. When choosing a frailty instrument, clinicians should consider accuracy and feasibility. Based on our review of seventy studies, strong evidence in both domains supports the Clinical Frailty Scale, while the Fried Phenotype may require a trade-off of accuracy with lower feasibility.

See supporting data below.

\section{REFERENCES:}

1. Rockwood K, Song X, MacKnight C, et al. A global clinical measure of fitness and frailty in elderly people. CMAJ. 2005;173(5):489-495. doi:10.1503/cmaj.050051.

2. Fried LP, Tangen CM, Walston J, et al. Frailty in older adults: evidence for a phenotype. J Gerontol A Biol Sci Med Sci. 2001;56(3).

3. Lin H-S, Watts JN, Peel NM, Hubbard RE. Frailty and post-operative outcomes in older surgical patients: a systematic review. BMC Geriatr. 2016;16(1):157. doi:10.1186/s12877016-0329-8.

4. Kim DH, Kim CA, Placide S, Lipsitz LA, Marcantonio ER. Preoperative Frailty Assessment and Outcomes at 6 Months or Later in Older Adults Undergoing Cardiac Surgical Procedures. Ann Intern Med. 2016. doi:10.7326/M16-0652.

5. Watt J, Tricco AC, Talbot-Hamon C, et al. Identifying older adults at risk of harm following elective surgery: a systematic review and meta-analysis. BMC Med. 2018;16(1):2. doi:10.1186/s12916-017-0986-2.

6. Mclsaac DI, Taljaard M, Bryson GL, et al. Frailty as a Predictor of Death or New Disability After Surgery: A Prospective Cohort Study. Ann Surg. 2018;accepted. doi:10.1097/SLA.0000000000002967.

7. Chow WB, Rosenthal RA, Merkow RP, et al. Optimal Preoperative Assessment of the Geriatric Surgical Patient: A Best Practices Guideline from the American College of Surgeons National Surgical Quality Improvement Program and the American Geriatrics Society. J Am Coll Surg. 2012;215(4):453-466. doi:10.1016/j.jamcollsurg.2012.06.017.

8. Association of Anaesthetists of Great Britain and Ireland. Peri-operative care of the elderly. Anaes. 2014;69 s1(January):81-98. doi:10.1111/anae.12524/abstract.

9. Alvarez-Nebreda ML, Bentov N, Urman RD, et al. Recommendations for Preoperative Management of Frailty from the Society for Perioperative Assessment and Quality Improvement (SPAQI). J Clin Anesth. 2018;47(February):33-42. doi:10.1016/j.jclinane.2018.02.011.

10. Eamer G, Gibson JA, Gillis C, et al. Surgical frailty assessment: a missed opportunity. BMC Anesthesiol. 2017;17(1):99. doi:10.1186/s12871-017-0390-7.

11. Stroup DF, Berlin J a, Morton SC, et al. Meta-analysis of observational studies in epidemiology: a proposal for reporting. Meta-analysis Of Observational Studies in Epidemiology (MOOSE) group. JAMA. 2000;283(15):2008-2012. doi:10.1001/jama.283.15.2008.

12. Higgins J, Green S. Cochrane Handbook for Systematic Reviews of Interventions Version 5.1.0 [updated March 2011]. Cochrane Collab. 2011. 
13. Shen $Y$, Hao Q, Zhou J, Dong B. The impact of frailty and sarcopenia on postoperative outcomes in older patients undergoing gastrectomy surgery: a systematic review and metaanalysis. BMC Geriatr. 2017;17(1):188. doi:10.1186/s12877-017-0569-2.

14. Lin H-S, Watts JN, Peel NM, Hubbard RE, Griebling TL. Re: Frailty and Post-Operative Outcomes in Older Surgical Patients: A Systematic Review. BMC. 2016;16(157). doi:10.1016/j.juro.2017.05.052.

15. Wang J, Zou Y, Zhao J, et al. The Impact of Frailty on Outcomes of Elderly Patients After Major Vascular Surgery: A Systematic Review and Meta-analysis. Eur J Vasc Endovasc Surg. August 2018. doi:10.1016/j.ejvs.2018.07.012.

16. Moher D, Liberati A, Tetzlaff J, Altman DG. Preferred Reporting Items for Systematic Reviews and Meta-Analyses: The PRISMA Statement. 2009;151(4):264-269.

17. Oakland K, Nadler R, Cresswell L, Jackson D, Coughlin PA. Systematic review and metaanalysis of the association between frailty and outcome in surgical patients. Ann $R$ Coll Surg Engl. January 2016:1-6. doi:10.1308/rcsann.2016.0048.

18. Watt J, Tricco AC, Talbot-Hamon C, et al. Identifying Older Adults at Risk of Delirium Following Elective Surgery: A Systematic Review and Meta-Analysis. J Gen Intern Med. 2018;33(4):500-509. doi:10.1007/s11606-017-4204-x.

19. Sampson M, McGowan J, Lefebvre C, Moher D, Grimshaw JM. PRESS: Peer Review of Electronic Search Strategies.; 2008. doi:10.1111/1540-4560.00063.

20. Mclsaac DI, Huang A, Wong CA, Wijeysundera DN, Bryson GL, van Walraven C. Effect of Preoperative Geriatric Evaluation on Outcomes After Elective Surgery: A Population-Based Study. J Am Geriatr Soc. 2017;65(12). doi:10.1111/jgs.15100.

21. Fried LP, Ferrucci L, Darer J, Williamson JD, Anderson G. Untangling the concepts of disability, frailty, and comorbidity: implications for improved targeting and care. J Gerontol A Biol Sci Med Sci. 2004;59(3):255-263.

22. Rodríguez-Mañas L, Féart C, Mann G, et al. Searching for an operational definition of frailty: A delphi method based consensus statement. the frailty operative definitionconsensus conference project. Journals Gerontol - Ser A Biol Sci Med Sci. 2013;68(1):6267. doi:10.1093/gerona/gls119.

23. Akpan A, Roberts C, Bandeen-Roche K, et al. Standard set of health outcome measures for older persons. BMC Geriatr. 2018;18(1):36.

24. Bowen DJ, Kreuter M, Spring B, et al. How We Design Feasibility Studies. Am J Prev Med. 2009;36(5):452-457. doi:10.1016/j.amepre.2009.02.002.

25. DistillerSR | Systematic Review and Literature Review Software by Evidence Partners.

26. Comprehensive Meta-Analysis Software (CMA).

27. Harris EP, MacDonald DB, Boland L, Boet S, Lalu MM, Mclsaac DI. Personalized perioperative medicine: a scoping review of personalized assessment and communication of risk before surgery. Can J Anaesth. June 2019. doi:10.1007/s12630-019-01432-6.

28. Wan X, Wang W, Liu J, Tong T. Estimating the sample mean and standard deviation from the sample size, median, range and/or interquartile range. BMC Med Res Methodol. 2014;14(1):135. doi:10.1186/1471-2288-14-135.

29. Hayden JA, van der Windt DA, Cartwright JL, Côté P, Bombardier C. Assessing bias in studies of prognostic factors. Ann Intern Med. 2013;158(4):280-286. doi:10.7326/00034819-158-4-201302190-00009.

30. Cooper Z, Rogers SO, Ngo L, et al. Comparison of Frailty Measures as Predictors of Outcomes After Orthopedic Surgery. J Am Geriatr Soc. 2016;64(12):2464-2471. doi:http://dx.doi.org/10.1111/jgs.14387. 
31. Courtney-Brooks M, Tellawi AR, Scalici J, et al. Frailty: An outcome predictor for elderly gynecologic oncology patients. Gynecol Oncol. 2012;126(1):20-24. doi:http://dx.doi.org/10.1016/j.ygyno.2012.04.019.4

32. Garonzik-Wang JM, Govindan P, Grinnan JW, et al. Frailty and delayed graft function in kidney transplant recipients. Arch Surg. 2012;147(2):190-193. doi:https://dx.doi.org/10.1001/archsurg.2011.1229.

33. Gleason LJ, Benton EA, Alvarez-Nebreda ML, Weaver MJ, Harris MB, Javedan H. FRAIL Questionnaire Screening Tool and Short-Term Outcomes in Geriatric Fracture Patients. J Am Med Dir Assoc. 2017;18(12):1082-1086. doi:http://dx.doi.org/10.1016/j.jamda.2017.07.005.

34. Han B, Wang Y, Chen X. Predictive value of frailty on postoperative complications in elderly patients with major abdominal surgery. Biomed Res. 2018;29(7):1308-1315.

35. Huded CP, Huded JM, Friedman JL, et al. Frailty Status and Outcomes after Transcatheter Aortic Valve Implantation. Am J Cardiol. 2016;117(12):1966-1971. doi:http://dx.doi.org/10.1016/j.amjcard.2016.03.044.

36. Jha SR, Hannu MK, Newton PJ, et al. Reversibility of Frailty After Bridge-to-Transplant Ventricular Assist Device Implantation or Heart Transplantation. Transplant direct. 2017;3(7):e167. doi:https://dx.doi.org/10.1097/TXD.0000000000000690.

37. Kapoor A, Matheos T, Walz M, et al. Self-Reported Function More Informative than Frailty Phenotype in Predicting Adverse Postoperative Course in Older Adults. J Am Geriatr Soc. 2017;65(11):2522-2528. doi:http://dx.doi.org/10.1111/jgs.15108.

38. Katlic MR, Coleman J, Khan K, Wozniak SE, Abraham JH. Sinai Abbreviated Geriatric Evaluation: Development and Validation of a Practical Test. Ann Surg. 2017. doi:https://dx.doi.org/10.1097/SLA.0000000000002597.

39. Khan SA, Chua HW, Hirubalan P, Karthekeyan RB, Kothandan H. Association between frailty, cerebral oxygenation and adverse post-operative outcomes in elderly patients undergoing non-cardiac surgery: An observational pilot study. Indian J Anaesth. 2016;60(2):102-107. doi:https://dx.doi.org/10.4103/0019-5049.176278.

40. Kotajarvi BR, Schafer MJ, Atkinson EJ, et al. The Impact of Frailty on Patient-Centered Outcomes Following Aortic Valve Replacement. J Gerontol A Biol Sci Med Sci. 2017;72(7):917-921. doi:http://dx.doi.org/10.1093/gerona/glx038.

41. Kristjansson SR, Ronning B, Hurria A, et al. A comparison of two pre-operative frailty measures in older surgical cancer patients. J Geriatr Oncol. doi:http://dx.doi.org/10.1016/j.jgo.2011.09.002.

42. Leung JM, Tsai TL, Sands LP. Preoperative frailty in older surgical patients is associated with early postoperative delirium. Anesth Analg. 2011;112(5):1199-1201. doi:http://dx.doi.org/10.1213/ANE.0b013e31820c7c06.

43. Li JL, Henderson MA, Revenig LM, et al. Frailty and one-year mortality in major intraabdominal operations. J Surg Res. 2016;203(2):507-512.e1. doi:https://dx.doi.org/10.1016/j.jss.2016.03.007.

44. Makary MA, Segev DL, Pronovost PJ, et al. Frailty as a Predictor of Surgical Outcomes in Older Patients. ACS. 2010;210(6):901-908. doi:10.1016/j.jamcollsurg.2010.01.028.

45. Makhani SS, Kim FY, Liu Y, et al. Cognitive Impairment and Overall Survival in Frail Surgical Patients. J Am Coll Surg. 2017;225(5):590-600.e1. doi:10.1016/j.jamcollsurg.2017.07.1066.

46. Pelavski AD, Miguel M De, Garcia-Tejedor GA, et al. Mortality, Geriatric, and Nongeriatric Surgical Risk Factors among the Eldest Old: A Prospective Observational Study. Anesth Analg. 2017;125(4):1329-1336. doi:http://dx.doi.org/10.1213/ANE.0000000000002389. 
47. Revenig LM, Canter DJ, Kim S, et al. Report of a Simplified Frailty Score Predictive of Short-Term Postoperative Morbidity and Mortality. J Am Coll Surg. 2015;220(5):904-911.e1. doi:10.1016/j.jamcollsurg.2015.01.053.

48. Revenig LM, Canter DJ, Taylor MD, et al. Too Frail for Surgery ? Initial Results of a Large Multidisciplinary Prospective Study Examining Preoperative Variables Predictive of Poor Surgical Outcomes. J Am Coll Surg. 2013;217(4):665-670.e1. doi:10.1016/j.jamcollsurg.2013.06.012.

49. Revenig LM, Canter DJ, Master VA, et al. A prospective study examining the association between preoperative frailty and postoperative complications in patients undergoing minimally invasive surgery. J Endourol. 2014;28(4):476-480. doi:http://dx.doi.org/10.1089/end.2013.0496.

50. Singer JP, Diamond JM, Anderson MR, et al. Frailty phenotypes and mortality after lung transplantation: A prospective cohort study. Am J Transplant. 2018;18(8):1995-2004. doi:http://dx.doi.org/10.1111/ajt.14873.

51. Kua J, Ramason R, Rajamoney G, Chong M, Chong MS. Which frailty measure is a good predictor of early post-operative complications in elderly hip fracture patients? Arch Orthop Trauma Surg. 2016;136(5):639-647. doi:10.1007/s00402-016-2435-7.

52. Tan KY, Kawamura YJ, Tokomitsu A, Tang T. Assessment for frailty is useful for predicting morbidity in elderly patients undergoing colorectal cancer resection whose comorbidities are already optimized. Am J Surg. 2012;204(2):139-143. doi:http://dx.doi.org/10.1016/j.amjsurg.2011.08.012.

53. Wang HT, Fafard J, Ahern S, Vendittoli P-A, Hebert P. Frailty as a predictor of hospital length of stay after elective total joint replacements in elderly patients. BMC Musculoskelet Disord. 2018;19(1):14. doi:10.1186/s12891-018-1935-8.

54. Lytwyn J, Stammers AN, Kehler DS, et al. The impact of frailty on functional survival in patients 1 year after cardiac surgery. J Thorac Cardiovasc Surg. 2017;154(6):1990-1999. doi:http://dx.doi.org/10.1016/j.jtcvs.2017.06.040.

55. Sikder T, Sourial N, Maimon G, et al. Postoperative Recovery in Frail, Pre-frail, and Nonfrail Elderly Patients Following Abdominal Surgery. World J Surg. 2018. doi:https://dx.doi.org/10.1007/s00268-018-4801-9.

56. Ad N, Holmes SD, Halpin L, Shuman DJ, Miller CE, Lamont D. The Effects of Frailty in Patients Undergoing Elective Cardiac Surgery. J Card Surg. 2016;31(4):187-194. doi:10.1111/jocs.12699.

57. Afilalo J, Lauck S, Kim DH, et al. Frailty in Older Adults Undergoing Aortic Valve Replacement: The FRAILTY-AVR Study. J Am Coll Cardiol. 2017;70(6):689-700. doi:http://dx.doi.org/10.1016/j.jacc.2017.06.024.

58. Andreou A, Lasithiotakis K, Venianaki M, et al. A Comparison of Two Preoperative Frailty Models in Predicting Postoperative Outcomes in Geriatric General Surgical Patients. World J Surg. 2018. doi:https://dx.doi.org/10.1007/s00268-018-4734-3.

59. Brown $\mathrm{CH}$, Max L, Laflam A, et al. The Association between Preoperative Frailty and Postoperative Delirium after Cardiac Surgery. Anesth Analg. 2016;123(2):430-435. doi:http://dx.doi.org/10.1213/ANE.0000000000001271.

60. McGuckin DG, Mufti S, Turner DJ, Bond C, Moonesinghe SR. The association of perioperative scores, including frailty, with outcomes after unscheduled surgery. Anaesthesia. 2018;73(7):819-824. doi:10.1111/anae.14269.

61. Donald GW, Ghaffarian AA, Isaac F, et al. Preoperative frailty assessment predicts loss of independence after vascular surgery. J Vasc Surg. 2018;68(5):1382-1389.

doi:http://dx.doi.org/10.1016/j.jvs.2018.02.044. 
62. Li S, Nie Y, Zhan J, et al. The analysis of correlation between frailty index and postoperative complications of aged patients with nodular goiter. Aging Med. 2018;1(1):1822. doi:http://dx.doi.org/10.1002/agm2.12016.

63. Goeteyn J, Evans LA, Cleyn S De, et al. Frailty as a predictor of mortality in the elderly emergency general surgery patient. Acta Chir Belg. 2017;117(6):370-375. doi:http://dx.doi.org/10.1080/00015458.2017.1337339.

64. Rodrigues MK, Marques A, Lobo DML, Umeda IIK, Oliveira MF. Pre-frailty increases the risk of adverse events in older patients undergoing cardiovascular surgery. Arq Bras Cardiol. 2017;109(4):299-306. doi:http://dx.doi.org/10.5935/abc.20170131.

65. Kovacs J, Moraru L, Antal K, Cioc A, Voidazan S, Szabo A. Are frailty scales better than anesthesia or surgical scales to determine risk in cardiac surgery? Korean J Anesthesiol. 2017;70(2):157-162. doi:http://dx.doi.org/10.4097/kjae.2017.70.2.157.

66. Reichart D, Rosato S, Nammas W, et al. Clinical frailty scale and outcome after coronary artery bypass grafting. Eur J Cardio-Thoracic Surg. 2018;0(February):1-8. doi:10.1093/ejcts/ezy222.

67. Sato T, Hatakeyama S, Okamoto T, et al. Slow Gait Speed and Rapid Renal Function Decline Are Risk Factors for Postoperative Delirium after Urological Surgery. PLoS One. 2016;11(5):e0153961. doi:http://dx.doi.org/10.1371/journal.pone.0153961.

68. Afilalo J, Eisenberg MJ, Morin JF, et al. Gait speed as an incremental predictor of mortality and major morbidity in elderly patients undergoing cardiac surgery. J Am Coll Cardiol. 2010;56(20):1668-1676. doi:http://dx.doi.org/10.1016/j.jacc.2010.06.039.

69. Ugolini G, Pasini F, Ghignone F, et al. How to select elderly colorectal cancer patients for surgery: a pilot study in an Italian academic medical center. Cancer Biol Med. 2015;12(4):302-307. doi:http://dx.doi.org/10.7497/j.issn.2095-3941.2015.0084.

70. Afilalo J, Kim S, O'Brien S, et al. Gait speed and operative mortality in older adults following cardiac surgery. JAMA Cardiol. 2016;1(3):314-321. doi:http://dx.doi.org/10.1001/jamacardio.2016.0316.

71. Chauhan D, Haik N, Merlo A, et al. Quantitative increase in frailty is associated with diminished survival after transcatheter aortic valve replacement. Am Heart J. 2016;182:146154. doi:http://dx.doi.org/10.1016/j.ahj.2016.06.028.

72. Itoh S, Yoshizumi T, Sakata K, et al. Slow Gait Speed Is a Risk Factor for Complications After Hepatic Resection. J Gastrointest Surg. 2018. doi:https://dx.doi.org/10.1007/s11605018-3993-5.

73. Madbouly K, AlHajeri D, Habous M, Binsaleh S. Association of the modified frailty index with adverse outcomes after penile prosthesis implantation. Aging Male. 2017;20(2):119124. doi:10.1080/13685538.2017.1292499.

74. Joseph B, Zangbar B, Pandit V, et al. Emergency General Surgery in the Elderly: Too Old or Too Frail? J Am Coll Surg. 2016;222(5):805-813. doi:https://dx.doi.org/10.1016/j.jamcollsurg.2016.01.063.

75. Lin H, Peel NM, Scott IA, et al. Perioperative assessment of older surgical patients using a frailty index-feasibility and association with adverse post-operative outcomes. Anaesth Intensive Care. 2017;45(6):676-682. doi:10.1177/0310057X1704500605.

76. Saxton A, Velanovich V. Preoperative frailty and quality of life as predictors of postoperative complications. Ann Surg. 2011;253(6):1223-1229. doi:http://dx.doi.org/10.1097/SLA.0b013e318214bce7.

77. Saricaoglu F, Aksoy SM, Yilmazlar A, et al. Predicting mortality and morbidity of geriatric femoral fractures using a modified frailty index and perioperative features: A prospective, multicentre and observational study. Turk Geriatr Derg. 2018;21(2):118-127. doi:http://dx.doi.org/10.31086/tjgeri.2018240413. 
78. Vasu BK, Ramamurthi KP, Rajan S, George M. Geriatric Patients with Hip Fracture: Frailty and Other Risk Factors Affecting the Outcome. Anesth essays Res. 2018;12(2):546-551. doi:https://dx.doi.org/10.4103/aer.AER_61_18.

79. Dunlay SM, Park SJ, Joyce LD, et al. Frailty and outcomes after implantation of left ventricular assist device as destination therapy. J Hear Lung Transplant. 2014;33(4):359365. doi:http://dx.doi.org/10.1016/j.healun.2013.12.014.

80. Partridge JSL, Fuller M, Harari D, Taylor PR, Martin FC, Dhesi JK. Frailty and poor functional status are common in arterial vascular surgical patients and affect postoperative outcomes. Int J Surg. 2015;18:57-63. doi:http://dx.doi.org/10.1016/j.ijsu.2015.04.037.

81. Cheung SC, Ahmad LA, Hardy JE, Hilmer SN. A prospective cohort study of older surgical inpatients examining the prevalence and implications of frailty. J Clin Gerontol Geriatr. 2017;8(2):71-76. doi:http://dx.doi.org/10.24816/jcgg.2017.v8i2.06.

82. Dasgupta M, Rolfson DB, Stolee P, Borrie MJ, Speechley M. Frailty is associated with postoperative complications in older adults with medical problems. Arch Gerontol Geriatr. 2009;48(1):78-83. doi:http://dx.doi.org/10.1016/j.archger.2007.10.007.

83. Amabili P, Wozolek A, Noirot I, et al. The Edmonton Frail Scale Improves the Prediction of 30-Day Mortality in Elderly Patients Undergoing Cardiac Surgery: A Prospective Observational Study. J Cardiothorac Vasc Anesth. 2018. doi:http://dx.doi.org/10.1053/j.jvca.2018.05.038.

84. Moro FD, Morlacco A, Motterle G, Barbieri L, Zattoni F. Frailty and elderly in urology: Is there an impact on post-operative complications? Cent Eur J Urol. 2017;70(2):197-205. doi:http://dx.doi.org/10.5173/ceju.2017.1321.

85. Kim S, Marsh AP, Rustowicz L, et al. Self-reported mobility in older patients predicts early postoperative outcomes after elective noncardiac surgery. Anesthesiology. 2016;124(4):815-825. doi:http://dx.doi.org/10.1097/ALN.0000000000001011.

86. Jokar TO, Ibraheem $\mathrm{K}$, Rhee $\mathrm{P}$, et al. Emergency general surgery specific frailty index: A validation study. J Trauma Acute Care Surg. 2016;81(2):254-260. doi:10.1097/TA.0000000000001120.

87. Pol RA, Leeuwen BL Van, Visser L, et al. Standardised Frailty Indicator as Predictor for Postoperative Delirium after Vascular Surgery: A Prospective Cohort Study. Eur J Vasc Endovasc Surg. 2011;42(6):824-830. doi:10.1016/j.ejvs.2011.07.006.

88. Robinson TN, Wu DS, Pointer L, Dunn CL, Jr JCC, Moss M. Simple frailty score predicts postoperative complications across surgical specialties. Am J Surg. 2013;206(4):544-550. doi:http://dx.doi.org/10.1016/j.amjsurg.2013.03.012.

89. Sundermann SH, Dademasch A, Seifert B, et al. Frailty is a predictor of short- and mid-term mortality after elective cardiac surgery independently of age. Interact Cardiovasc Thorac Surg. 2014;18(5):580-585. doi:http://dx.doi.org/10.1093/icvts/ivu006.

90. Tanaka $\mathrm{S}$, Ueno $\mathrm{M}$, lida $\mathrm{H}$, et al. Preoperative assessment of frailty predicts age-related events after hepatic resection: a prospective multicenter study. $J$ Hepatobiliary Pancreat Sci. 2018;25(8):377-387. doi:http://dx.doi.org/10.1002/jhbp.568.

91. Tegels JJW, Maat MFG de, Hulsewe KWE, Hoofwijk AGM, Stoot JHMB. Value of Geriatric Frailty and Nutritional Status Assessment in Predicting Postoperative Mortality in Gastric Cancer Surgery. J Gastrointest Surg. 2014;18(3):439-446. doi:http://dx.doi.org/10.1007/s11605-013-2443-7.

92. Windt DJ van der, Bou-Samra P, Dadashzadeh ER, Chen X, Varley PR, Tsung A. Preoperative risk analysis index for frailty predicts short-term outcomes after hepatopancreatobiliary surgery. HPB. 2018. doi:http://dx.doi.org/10.1016/j.hpb.2018.05.016. 
93. Choi JY, Yoon SJ, Jung HW, et al. Prediction of Postoperative Complications Using Multidimensional Frailty Score in Older Female Cancer Patients with American Society of Anesthesiologists Physical Status Class 1 or 2. J Am Coll Surg. 2015;221(3):652-660. doi:http://dx.doi.org/10.1016/j.jamcollsurg.2015.06.011.

94. Hall DE, Arya S, Schmid KK, et al. Development and initial validation of the Risk Analysis Index for measuring frailty in surgical populations. JAMA Surg. 2017;152(2):175-182. doi:http://dx.doi.org/10.1001/jamasurg.2016.4202.

95. Kenig J, Mastalerz K, Lukasiewicz K, Mitus-Kenig M, Skorus U. The Surgical Apgar Score predicts outcomes of emergency abdominal surgeries both in fit and frail older patients. Arch Gerontol Geriatr. 2018;76:54-59. doi:http://dx.doi.org/10.1016/j.archger.2018.02.001.

96. Lu J, Zheng HL, Li P, et al. High preoperative modified frailty index has a negative impact on short- and long-term outcomes of octogenarians with gastric cancer after laparoscopic gastrectomy. Surg Endosc Other Interv Tech. 2018;32(5):2193-2200. doi:http://dx.doi.org/10.1007/s00464-018-6085-4.

97. Marshall L, Griffin R, Mundy J. Frailty assessment to predict short term outcomes after cardiac surgery. Asian Cardiovasc Thorac Ann. 2016;24(6):546-554. doi:http://dx.doi.org/10.1177/0218492316653557.

98. Botto F, Alonso-Coello P, Chan MT V., et al. Myocardial Injury after Noncardiac Surgery. Anesthesiology. 2014;120(3):564-578. doi:10.1097/ALN.0000000000000113.

99. Li Y, Pederson J, Churchill T, et al. Impact of frailty on outcomes after discharge in older surgical patients: a prospective cohort study. CMAJ. 2018;190(7):184-190. doi:10.1503/cmaj.161403.

100. Orouji Jokar $\mathrm{T}$, Ibraheem $\mathrm{K}$, Rhee $\mathrm{P}$, et al. Emergency general surgery specific frailty index: A validation study AAST Continuing Medical Education Article Accreditation Statement. doi:10.1097/TA.0000000000001120.

101. DE, Arya S, Schmid KK, et al. Association of a Frailty Screening Initiative With Postoperative Survival at 30, 180, and 365 Days. JAMA Surg. 2017;152(3):233. doi:10.1001/jamasurg.2016.4219.

102. Clavien PA, Strasberg SM. Severity grading of surgical complications. Ann Surg. 2009;250(2):197-198. doi:10.1097/SLA.0b013e3181b6dcab.

103. National Surgical Quality Improvement Program Database.; 2013.

104. Walker EMK, Bell M, Cook TM, et al. Patient reported outcome of adult perioperative anaesthesia in the United Kingdom: a cross-sectional observational study. Br J Anaesth. 2016;117(6):758-766. doi:10.1093/bja/aew381.

105. Hewitt J, Long S, Carter B, Bach S, McCarthy K, Clegg A. The prevalence of frailty and its association with clinical outcomes in general surgery: A systematic review and metaanalysis. Age Ageing. 2018;47(6):793-800. doi:10.1093/ageing/afy110.

106. Sepehri A, Beggs $T$, Hassan A, et al. The impact of frailty on outcomes after cardiac surgery: A systematic review. J Thorac Cardiovasc Surg. 2014;148(6):3110-3117. doi:10.1016/j.jtcvs.2014.07.087.

107. Association of Anaesthetists of Great Britain and Ireland. Peri-operative care of the elderly 2014. Anaesthesia. 2014;69(s1):81-98. doi:10.1111/anae.12524/abstract.

108. Watt J, Tricco AC, Talbot-Hamon C, et al. Identifying Older Adults at Risk of Harm Following Elective Surgery: A Systematic Review and Meta-Analysis. J Gen Intern Med. 2018;33(4):500-509. doi:10.1007/s11606-017-4204-X.

109. Hempel S, Miles JN V, Booth MJ, Wang Z, Morton SC, Shekelle PG. Risk of bias: a simulation study of power to detect study-level moderator effects in meta-analysis. Syst Rev. 2013;2:107. doi:10.1186/2046-4053-2-107. 
110. Fried TR, Bradley EH, Towle VR, Allore H. Understanding the treatment preferences of seriously ill patients. N Engl J Med. 2002;346(14):1061-1066.

doi:10.1056/NEJMsa012528.

111. Measuring Frailty in Critical Illness: Province-Wide Implementation of the Clinical Frailty Scale Score in a Population-Based Electronic Medical Record System in Alberta.

https://www.ualberta.ca/critical-care/research/current-research/cfs-implementation. Accessed June 28, 2019.

112. Steyerberg EW, Vickers AJ, Cook NR, et al. Assessing the performance of prediction models : A framework for some traditional and novel measures. Epidemiology. 2010;21(1):128-138. doi:10.1097/EDE.0b013e3181c30fb2.Assessing.

113. Robinson TN, Walston JD, Brummel NE, et al. Frailty for Surgeons: Review of a National Institute on Aging Conference on Frailty for Specialists. J Am Coll Surg.

2015;221(6):1083-1092. Doi 
a)

\section{Association of Frailty Phenotype with Mortality}

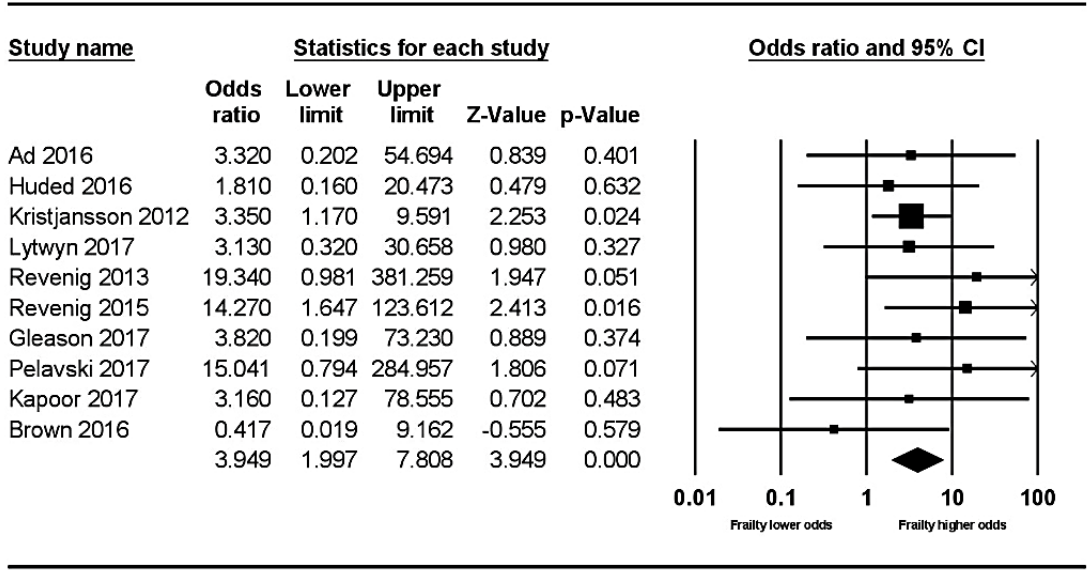

b)

\section{Association of Clinical Frailty Scale with Mortality}

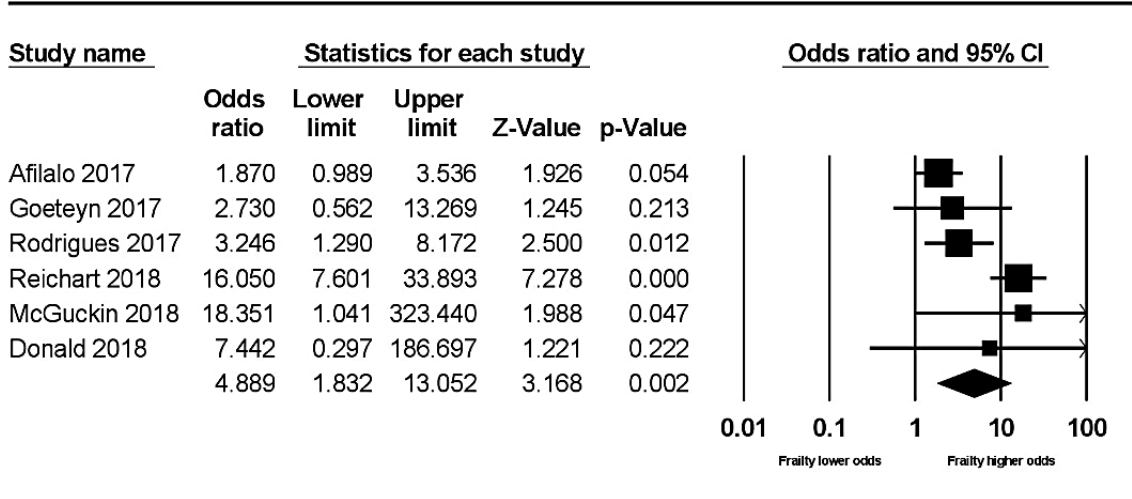

c)

\section{Association of Physical Frailty with Mortality}

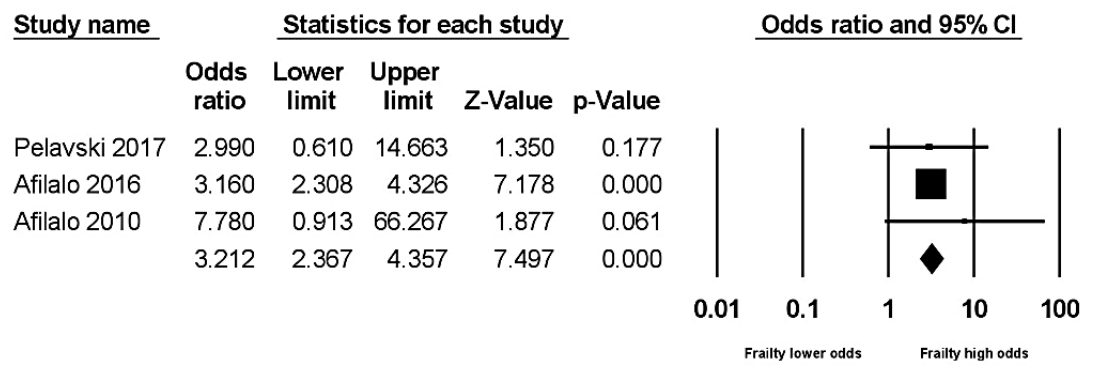




\title{
RICHARD KNILL COMPETITION
}

\author{
Cognitive and Behavioral Changes Following Exposure to Either Sevoflurane- or \\ Propofol-Based Anesthesia in Children Undergoing MRI
}

Jillian Miller ${ }^{1}$, Allison McPeak ${ }^{2}$; Adam Spencer ${ }^{1}$; Nivez Rasic ${ }^{1}$; Melanie Noel ${ }^{3}$; Kailyn Jones ${ }^{4}$, Tasmia Hai ${ }^{5}$; Kendra Epping; ${ }^{4}$ Andrew Walker ${ }^{1}$; Chad Bousman'; Naweed Syed ${ }^{7}$; Frank P. MacMaster $^{8,9}$; Tiffany Rice ${ }^{1}$

1 Anesthesiology, Perioperative and Pain Medicine, University of Calgary, Calgary, Canada

2 Medicine, University of Calgary, Calgary, Canada

3 Psychology, University of Calgary, Calgary, Canada

4 Werklund School of Education, University of Calgary, Calgary, Canada

5 School Psychology, University of Alberta, Edmonton, Canada

6 Medical Genetics, University of Calgary, Calgary, Canada

7 Cell Biology and Anatomy, University of Calgary, Calgary, Canada

8 Psychiatry and Pediatrics, University of Calgary, Calgary, Canada

9 Addictions and Mental Health Strategic Clinical Network

Introduction: Pediatric patients often require general anesthesia (GA) for magnetic resonance imaging (MRI). The effect of anesthetic agents on neurodevelopmental outcomes is unclear. Animal data supports detrimental effects, while human data has been inconsistent (1). Our study examined the effect of exposure to sevoflurane or propofol on cognitive and behavioral outcomes in children undergoing MRI.

Methods: Ethics approval was obtained from the local Research Ethics Board (REB16-0104). This study included children $(\mathrm{N}=50)$ between the ages of two and five years (American Society of Anesthesiologists Classification I-II), requiring GA for MRI. Prior to the MRI, parents completed reliable and validated questionnaires regarding their child's cognition (Behaviour Rating Inventory of Executive Function, Preschool Version [BRIEF-P]) (2) and behavior (Child Behavior Checklist 1.5-5 [CBCL]) (3). The BRIEF-P consists of 63 items rated on a Likert scale ranging from one (never) to three (often) that measures executive functioning across five domains: Inhibit, Shift, Emotional Control, Working Memory, and Planning/Organization, and forms one composite score (Global Executive Composite). The CBCL consists of 99 items rated on a Likert scale ranging from zero (not true) to two (very true or often true). The CBCL forms two broad domains, Internalizing (anxious, depressive, withdrawn and somatic symptoms) and Externalizing Problems (attention problems and aggressive behavior). Children were randomized via block design to receive either a sevoflurane- or propofol-based anesthetic. No long acting opioids or nitrous oxide were used. Airway management was at the discretion of the anesthesiologist. Questionnaires were repeated 90 days post-MRI. Linear mixed models were used to compare Global Executive Composite, Internalizing and Externalizing scores between children exposed to either sevoflurane- or propofol-based anesthesia. Paired t-tests were used to compare baseline to 90-day post-MRI Global Executive Composite, Internalizing and Externalizing scores in combined sevoflurane- and propofol-exposed groups.

Results: No differences in outcomes were observed between children exposed to sevofluraneor propofol-based anesthesia. However, exposure to anesthetic (either sevoflurane or propofol) was associated with a moderate change in Global Executive Composite ( $t=-1.99$ [37], $P=0.054$, Cohen's $d=0.32$ ). Changes in the Global Executive Composite score were driven by changes in Working Memory ( $t=-2.09$ [37], $P=0.043$, Cohen's $d=0.34)$, and Planning/Organization ( $t=-$ 2.25(37), $P=0.031$, Cohen's $d=0.37$ ). Moreover, Internalizing scores were moderately changed 
( $t=-2.01[38], P=0.051$, Cohen's $d=0.32$ ), and Externalizing scores were significantly changed ( $t=-2.37[37], P=0.023$, Cohen's $d=0.38$ ), following exposure to GA for MRI.

Conclusions: Exposure to GA for MRI, regardless of anesthetic type, was associated with worsening cognitive and behavioral outcomes in preschool-aged children. Future research utilizing a larger sample size is needed to determine whether these changes persist beyond 90 days post-MRI. Based on the cognitive and behavioral changes reported by parents, it may be advisable to reduce the frequency of MRIs and minimize anesthetic exposure in children less than 5 years of age.

\section{REFERENCES:}

1. Iqbal et al. (2019) JNP 122(2):760-87; 2) Gioia et al. (2003) Assessment Resources: Lutz, FL; 3) Achenbach \& Rescorla (2000) Research Center for Children, Youth, and Families: Burlington, VT; 4) Thieba et al. (2008) Front Pediatr 6:146. 


\section{Competence of Anesthesiology Residents Following a Longitudinal Point-of-Care} Ultrasound Curriculum: Early Results

$\underline{\text { Michelle Clunie }}^{1}$, Jennifer O’Brien ${ }^{1}$, Paul Olszynski $^{2}$, Jagmeet Bajwa ${ }^{1}$, Robert Perverseff ${ }^{1}$

1 Department of Anesthesiology, Perioperative Medicine and Pain Management, University of Saskatchewan, Saskatoon, Canada

2 Department of Emergency Medicine, University of Saskatchewan, Saskatoon, Canada

Introduction: Point-of-care ultrasound (POCUS) facilitates many diagnostic and procedural applications in anesthesiology. ${ }^{1-3}$ Structured POCUS curricula have been shown to improve learner satisfaction, test scores, and affect clinical management, ${ }^{4}$ but the learning curve towards competency and retention of competency over time remain unknown.

Methods: Ethics approval was waived by the local REB. We conducted a prospective observational study to determine whether anesthesiology trainees enrolled in a POCUS curriculum can achieve competency in POCUS skills and to plot the learning curve of trainees' competency using a POCUS-specific competency-based medical education assessment. The structured POCUS curriculum comprised of online lectures, ${ }^{5}$ journal articles, live model scanning sessions, video review of cases, and development of a portfolio of supervised scans was delivered to residents in the Foundations and Core stages of training (PGY2-4) between January 2019 and February 2020. POCUS applications included Rescue Echo (focused cardiac ultrasound + lung for pneumothorax), lung ultrasound, Focused Assessment with Sonography in Trauma (FAST), abdominal aorta (AA), airway, and gastric ultrasound. POCUS scanning sessions on standardized patients were conducted in the simulation lab for 2.5 hours weekly; each resident attended 8 sessions (20 hours) per academic year. Timed image acquisition scores were obtained at each session by an experienced sonographer. Faculty evaluators completed the POCUS Skills Entrustment Scale ${ }^{6}$ at each scanning session. Final entrustment scores reflected the lowest score obtained in the domains of insight, image acquisition, image interpretation, and clinical integration. Primary outcome was number of sessions and scans required to achieve average entrustment scores $\geq 4$ ("may use independently"). Secondary outcomes include retention of skills at 3 months. The curriculum is ongoing.

Results: Twenty-five anesthesiology residents participated in the curriculum to date. An average POCUS entrustment score $\geq 4$ was achieved in Rescue Echo after 9 sessions/36 supervised scans ( 14.5 dedicated curricular hours), and in lung ultrasound after 2 sessions/12 supervised scans ( 2 dedicated curricular hours). Residents have achieved average POCUS entrustment scores of 3 ("may use with indirect supervision") in FAST after 2 sessions/8 supervised scans (2 dedicated hours), and in AA ultrasound after 2 sessions/8 supervised scans ( 1 dedicated hour). Residents have achieved average entrustment scores of 2 ("may use only under direct supervision") in airway ultrasound after 2 sessions/8 supervised scans ( 2 dedicated hours) and in gastric ultrasound after 2 sessions/8 supervised scans ( 1 dedicated hour).

Conclusion: Our preliminary results suggest that anesthesiology residents participating in a structured longitudinal POCUS curriculum can achieve competence in Rescue Echo after 36 supervised curricular scans on standardized patients, and lung ultrasound after 12 supervised 
scans. These findings will be further informed by the final aspect of the curriculum which will include observations and entrustment scores from clinically integrated POCUS scans and comprehensive summative assessments.

\section{REFERENCES:}

1. McCormick TJ, Miller EC, Chen R, Naik VN. Acquiring and maintaining point-of-care ultrasound (POCUS) competence for anesthesiologists. Can J Anesth. 2018 Apr. 65(4)42736.

2. The Association of Anaesthetists of Great Britain \& Ireland, The Royal College of Anaesthetists, The Intensive Care Society. Ultrasound in Anaesthesia and Intensive Care: A Guide to Training. London, UK: 2011. Available from: http://dx.doi.org/10.xx36/g.UIAAIC.2011

3. Mok D, Schwarz SKW, Rondi K. Point-of-care ultrasonography in Canadian anesthesiology residency programs: a national survey of program directors. Can J Anesth. 2017;64:102336.

4. Ramsingh D, Rinehart J, Kain Z, Strom S, Canales C, Alexander B, et al. Impact assessment of perioperative point-of-care ultrasound training on anesthesiology residents. Anesthesiology. 2015; 123(3):670-82.

5. Zimmerman J. Perioperative Echocardiography Education [Internet]. Salt Lake City, UT: The University of Utah; 2018. Cited 27 Nov 2018. Available from: https://echo.anesthesia.med.utah.edu/pocus-content/

6. Schnobich DJ, Mathews BK, Trappey BE, Muthyala BK, Olson APJ. Entrusting internal medicine residents to use points of care ultrasound: towards improved assessment and supervision. Med Teach. 2018; DOI: 10.1080/0142159X.2018.1457210

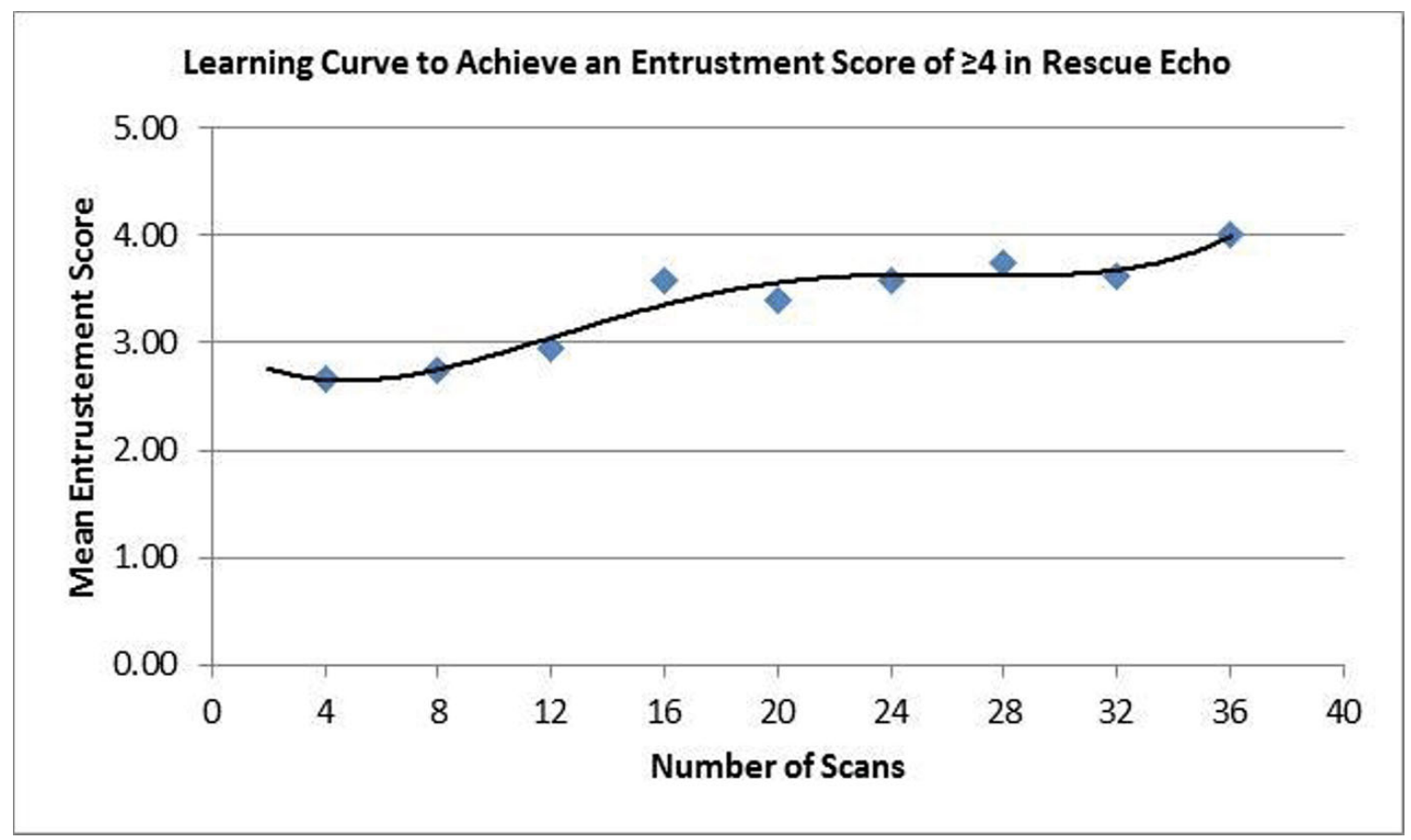




\section{Perioperative Outcomes of Nunavummiut Inuit in a Tertiary Care Hospital in Canada}

Justine M O'Shea ${ }^{1,2}$; Jason A McVicar ${ }^{1,2}$; Alana Poon ${ }^{1,2}$; Nadine R Caron ${ }^{5,6}$; M Dylan Bould ${ }^{1,3}$; Jason W Nickerson ${ }^{7,8}$; Jean Allen ${ }^{9}$; Michelle Doucet-Issaluk ${ }^{10}$; Donna May Kimmaliardjuk ${ }^{2,4}$; Chelsey Sheffield ${ }^{13}$; Caitlin Champion ${ }^{12,13}$; Daniel I Mclsaac ${ }^{1,2}$

1 Department of Anesthesiology and Pain Medicine, University of Ottawa, Ottawa, ON

2 The Ottawa Hospital, Ottawa, ON

3 Children's Hospital of Eastern Ontario, Ottawa, ON

4 Department of Surgery, University of Ottawa, Ottawa, ON

5 Department of Surgery and Northern Medical Program, University of British Columbia, Prince George, BC

6 Centre for Excellence in Indigenous Health, University of British Columbia, Vancouver, BC

7 Bruyère Research Institute, Ottawa, ON

8 Centre for Health Law, Policy and Ethics, University of Ottawa, Ottawa, ON

9 Nunavut Tunngavik Incorporated

10 Government of Nunavut

11 Qikiqtani General Hospital, Iqaluit, NU

12 West Parry Sound Health Centre, Parry Sound, ON

13 Department of Surgery, Northern Ontario School of Medicine, Sudbury, ON

Introduction \& Objectives: Structural aspect of the healthcare system, specifically limited access to surgical specialists and perioperative processes impact the outcomes and health resource use for Inuit surgical patients from Nunavut undergoing elective and emergency surgical procedures at a tertiary Canadian Hospital.

Methods: Ethics approval was received from the local Research Ethics Board. A retrospective cohort study was conducted of all inpatient surgery between 2011 and 2018 at a Canadian tertiary referral centre for eastern Nunavut. Primary outcome was a composite of in-hospital mortality or major complication. Secondary outcomes included postoperative hospital length of stay (LOS), readmissions within 30 days, institutional discharge and total hospitalization costs.

Results: There were 98,701 episodes of inpatient surgical care; $928(0.9 \%)$ involved patients with Nunavut Inuit status. 159 (17.2\%) of Nunavut Inuit patients died or suffered a postoperative complication, compared to 15,691 (16.1\%) of the control group. After adjustment, individuals with Inuit status were significantly more likely to experience the primary outcome (OR $1.25,95 \% \mathrm{Cl} 1.03$ to $1.51 ; \mathrm{P}=0.025$ ). In subgroup analyses, the largest increase in risk of death or major complication for Nunavut Inuit status was associated with cancer surgery (OR 1.63, 95\% Cl 1.03 to 2.58: $\mathrm{P}=0.039$ ) and elective surgery (OR 1.59, 95\% $\mathrm{Cl} 1.20$ to 2.10; $\mathrm{P}=$ 0.001 ). Readmission occurred in $8.4 \%$ of Inuit patients vs. $6.9 \%$ (OR $1.4,95 \% \mathrm{Cl} 1.09$ to 1.77 ; $\mathrm{P}=0.007$ ); adverse discharge occurred in $21.8 \%$ of Inuit patients vs. $12.9 \%$ (OR $2.2,95 \% \mathrm{Cl}$ 1.78 to 2.68; $p=<0.0001$ ); mean LOS for Inuit patients was 8.9 days vs. 6.6 days (difference 1.28; ratio of means 1.17 to 1.4 ); mean total cost for Inuit patients was $\$ 18,017$ vs. $\$ 14,704$ (difference 1.17; ratio of means 1.07 to 1.23 ).

Conclusions: Overall perioperative mortality and complications are $25 \%$ higher for Inuit patients from Nunavut at a major tertiary care hospital in Canada compared to the general population with increases in readmissions, adverse discharges, LOS and total cost. An overhaul of the perioperative systems involving multiple institutions and governments are required to address this inequity. 
Table 1: Unadjusted and adjusted outcomes

\begin{tabular}{|c|c|c|c|c|c|}
\hline & & $\begin{array}{c}\text { Inpatient surgical } \\
\text { episodes in } \\
\text { patients without } \\
\text { Inuit status } \\
\text { patients with Inuit } \\
\text { status } \\
\text { episodes in }\end{array}$ & $\begin{array}{c}\text { Inpatient surgical } \\
\text { effect* estimate }\end{array}$ & $\begin{array}{c}\text { Adjusted effect* } \\
\text { estimate }\end{array}$ \\
\hline $\begin{array}{c}\text { In-hospital } \\
\text { mortality or } \\
\text { major } \\
\text { complication }\end{array}$ & $\mathrm{n}(\%)$ & $15,691(16.1)$ & $159(17.2)$ & $1.06(0.89-1.27)$ & $1.25(1.03-1.51)$ \\
\hline Readmission & $\mathrm{n}(\%)$ & $6,582(6.9)$ & $76(8.4)$ & $1.27(0.99-1.62)$ & $1.4(1.09-1.77)$ \\
\hline $\begin{array}{c}\text { Adverse } \\
\text { Discharge }\end{array}$ & $\mathrm{n}(\%)$ & $12,065(12.9)$ & $202(21.8)$ & $1.84(1.56-2.17)$ & $2.18(1.78-2.68)$ \\
\hline LOS & Mean (SD) & $6.56(11.98)$ & $8.85(14.41)$ & & $1.28(1.17-1.4)$ \\
\hline Total Cost & Mean (SD) & $\$ 14,703(\$ 25,884)$ & $\$ 18,017(\$ 30,832)$ & & $1.17(1.07-1.23)$ \\
\hline
\end{tabular}

Note: $\mathrm{SD}$ = standard deviation; $\mathrm{Cl}$ = confidence interval; *Effect estimate for in-hospital mortality or major complication, readmission and adverse discharge is an odds ratio; for LOS and cost is ratio of means. 


\section{Prophylactic co-administration of oxytocin-ergonovine or oxytocin-carboprost versus oxytocin alone at cesarean delivery for labor arrest: A randomized controlled trial}

Mrinalini Balki $^{1,2,3}$; Kristi Downey¹; Andrew Walker; Gareth Seaward²; Jose Carvalho ${ }^{1,2}$

1 Department of Anesthesia and Pain Management, Mount Sinai Hospital, University of Toronto, Toronto, Canada

2 Department of Obstetrics and Gynaecology, Mount Sinai Hospital, University of Toronto, Toronto, Canada

3 The Lunenfeld-Tanenbaum Research Institute, Sinai Health System, Toronto, Canada 4 Department of Anesthesia, University of Calgary, Calgary, Alberta, Canada

Introduction: Women with labor arrest have a higher predisposition to postpartum hemorrhage during cesarean delivery (CD) compared to non-laboring women, due to desensitization of oxytocin receptors from prior exposure to oxytocin during labor. ${ }^{1}$ The objective of our study was to compare the efficacy of prophylactic administration of oxytocin-ergonovine (OE) vs. oxytocincarboprost $(\mathrm{OC})$ vs. oxytocin alone $(\mathrm{O})$ at $\mathrm{CD}$ for labor arrest.

Methods: Ethics approval was obtained from the local REB. This was a prospective, doubleblind, randomized controlled study on women undergoing CD for labor arrest under epidural analgesia. Women who received at least $4 \mathrm{~h}$ of oxytocin for labor augmentation were included. They were randomized into 3 groups and prophylactically administered the study drugs after fetal delivery: OE group received IV oxytocin 5IU + IV ergonovine $0.25 \mathrm{mg}$ and IM placebo $(1 \mathrm{~mL}$ NS); OC group received IV oxytocin 5IU and IM carboprost $0.25 \mathrm{mg}(1 \mathrm{~mL})$; and O group received IV oxytocin $5 \mathrm{IU}$ and IM placebo (1mL NS). Intravenous drugs, diluted in $10 \mathrm{~mL}$ saline, were administered over $1 \mathrm{~min}$. Immediately after the administration of study drugs, oxytocin $40 \mathrm{mU} / \mathrm{min}$ infusion was started in all groups. The obstetrician was asked to rate the uterine tone as satisfactory, equivocal or unsatisfactory at 3,5 and 10 min after delivery. Additional uterotonics, if needed, were given, as per a planned regimen. The primary outcome was intraoperative need for additional uterotonics. Secondary outcomes included uterine tone, calculated blood loss and side effects. Multivariate logistic regression model was used to predict the need for additional uterotonics. Linear regression was used to develop a predictive model of calculated blood loss, and uterine tone was assessed using generalized estimating equations.

Results: Total 100 women were recruited $(\mathrm{OE}=33, \mathrm{OC}=32, \mathrm{O}=35)$. They had similar baseline demographic and obstetrical characteristics. The mean (SD) duration of oxytocin infusion during labor was $13(7) \mathrm{h}$ and the maximum rate was $16(8) \mathrm{mU} / \mathrm{min}$. Additional uterotonics were required in $35 \%$ cases after a mean (SD) of 9 (5) min after delivery, and did not differ across groups $(p=0.94)$ (Table 1$)$. The blood loss was not different across groups. There was a trend towards higher incidence of hypotension in O group (40\%) compared to OE $(15 \%)$ or OC $(25 \%)$ $(p=0.07)$, with significantly higher need for phenylephrine in O compared to OE group (adjusted $p=0.004)$. Incidence of nausea and vomiting was not different across groups, however, there was a trend towards higher nausea in $\mathrm{OE}$ and $\mathrm{OC}$ groups than $\mathrm{O}$ group $(\mathrm{P}=0.06)$.

Discussion: We do not recommend the prophylactic use of combination of uterotonic drugs. Side effects were high in all groups, perhaps owing to high bolus doses of oxytocin. We suggest that oxytocin should be used as an infusion in appropriate doses and additional uterotonics used only as required. 


\section{REFERENCES:}

1. Balki, Tsen. Oxytocin protocols for cesarean delivery. Int Anesthesiol Clin 2014;52:48

Table 1. Primary and secondary outcomes across the study groups

\begin{tabular}{|c|c|c|c|c|}
\hline Outcomes & $\begin{array}{l}\text { Oxytocin+ } \\
\text { Ergonovine } \\
(\mathbf{n}=33)\end{array}$ & $\begin{array}{c}\text { Oxytocin+ } \\
\text { Carboprost } \\
(n=32)\end{array}$ & $\begin{array}{c}\text { Oxytocin } \\
(\mathbf{n}=35)\end{array}$ & P value \\
\hline Need for additional uterotonics $(\mathrm{n} \%)$ & $11(33.3 \%)$ & $11(34.4 \%)$ & $13(37.1 \%)$ & 0.943 \\
\hline More than one rescue uterotonic $(\mathrm{n} \%)$ & $6(18.2 \%)$ & $4(12.5 \%)$ & $2(5.7 \%)$ & 0.270 \\
\hline Rescue oxytocin $(\mathrm{n} \%)$ & $11(33.3 \%)$ & $11(34.4 \%)$ & $13(37.1 \%)$ & 0.943 \\
\hline Rescue ergonovine $(\mathrm{n} \%)$ & $4(12.1 \%)$ & $2(6.3 \%)$ & $0(0.0 \%)$ & 0.079 \\
\hline Rescue carboprost (n\%) & $4(12.1 \%)$ & $2(6.3 \%)$ & $2(5.7 \%)$ & 0.652 \\
\hline Rescue misoprostol (n\%) & $2(6.1 \%)$ & $2(6.3 \%)$ & $0(0.0 \%)$ & 0.387 \\
\hline $\begin{array}{l}\text { Time ( } \min \text { ) for first rescue (oxytocin) after delivery; } \\
\text { mean (SD) }\end{array}$ & $5.9(2.9)$ & $8.6(6.3)$ & $10.3(5.3)$ & 0.116 \\
\hline $\begin{array}{l}\text { Time (min) for second rescue after delivery } \\
\text { (ergonovine or carboprost); mean (SD) }\end{array}$ & $12.3(3.8)$ & $18.0(4.2)$ & $24.0(5.7)$ & 0.104 \\
\hline Satisfactory uterine tone at $3 \mathrm{~min}$ after delivery $(\mathrm{n} \%)$ & $19(57.6 \%)$ & $21(65.6 \%)$ & $22(62.9 \%)$ & 0.814 \\
\hline Satisfactory uterine tone at $5 \mathrm{~min}$ after delivery $(\mathrm{n} \%)$ & $23(69.7 \%)$ & $24(75.0 \%)$ & $23(65.7 \%)$ & 0.707 \\
\hline Satisfactory uterine tone at $10 \mathrm{~min}$ after delivery $(\mathrm{n} \%)$ & $24(72.7 \%)$ & $25(78.1 \%)$ & $29(82.9 \%)$ & 0.656 \\
\hline Uterine massage $(\mathrm{n} \%)$ & $12(36.4 \%)$ & $9(28.1 \%)$ & $9(25.7 \%)$ & 0.629 \\
\hline Calculated blood loss, mL; mean (SD) & $1145(596)$ & $1242(609)$ & $1180(504)$ & 0.789 \\
\hline \multicolumn{5}{|l|}{ Side effects } \\
\hline Hypotension $(<20 \%$ baseline; $\mathrm{n} \%)$ & $5(15.2 \%)$ & $8(25.0 \%)$ & $14(40.0 \%)$ & 0.068 \\
\hline Phenylephrine amount (mg/mL; median [IQR]) & $0.0 *[0.0$ to 0.2$]$ & $0.2[0.0$ to 0.4$]$ & $0.2[0.0$ to 0.6$]$ & 0.006 \\
\hline Hypertension ( $>20 \%$ baseline; $\mathrm{n} \%$ ) & $5(15.2 \%)$ & $7(21.9 \%)$ & $6(17.1 \%)$ & 0.805 \\
\hline Tachycardia (>30\% baseline; $\mathrm{n} \%$ ) & $11(32.3 \%)$ & $17(53.1 \%)$ & $17(48.6 \%)$ & 0.242 \\
\hline Chest pain $(\mathrm{n} \%)$ & $2(6.1 \%)$ & $1(3.1 \%)$ & $0(0 \%)$ & 0.311 \\
\hline $\mathrm{SpO}_{2}<90 \%(\mathrm{n} \%)$ & $0(0.0 \%)$ & $3(9.4 \%)$ & $2(5.7 \%)$ & 0.234 \\
\hline Shortness of breath $(\mathrm{n} \%)$ & $1(3.0 \%)$ & $1(3.1 \%)$ & $1(2.9 \%)$ & 1.000 \\
\hline Nausea $(\mathrm{n} \%)$ & $25(75.8 \%)$ & $21(65.6 \%)$ & $17(48.6 \%)$ & 0.059 \\
\hline Vomiting $(\mathrm{n} \%)$ & $8(24.2 \%)$ & $13(40.6 \%)$ & $7(20.0 \%)$ & 0.149 \\
\hline Need for antiemetics $(\mathrm{n} \%)$ & $19(57.6 \%)$ & $20(62.5 \%)$ & $17(48.6 \%)$ & 0.500 \\
\hline Headache $(\mathrm{n} \%)$ & $3(9.1 \%)$ & $3(9.4 \%)$ & $0(0.0 \%)$ & 0.161 \\
\hline Flushing (n\%) & $4(12.1 \%)$ & $4(12.5 \%)$ & $2(5.7 \%)$ & 0.598 \\
\hline
\end{tabular}

*Significant difference $(P=0.004)$ in phenylephrine administered post-delivery between oxytocin and oxytocin+ergonovine study groups. 
Recovery of Psychomotor Function in Patients after Ambulatory Surgery Under General Anaesthesia With and Without Anxiolytic Premedication - A Randomized Investigation

Taras Usichenko $^{1,2}$; Yacin Keller; Robert Lehmann4; Dragan Pavlovic ${ }^{5}$; Klaus Hahnenkamp; Andreas Nowak ${ }^{3}$

1 Dept. of Anesthesiology, University Medicine of Greifswald, Germany

2 Dept. of Anesthesia, McMaster University, Hamilton, Canada

3 Dept. of Anesthesiology and Intensive Care Medicine, Emergency Medicine and Pain

Management, Hospital Dresden-Friedrichstadt, Dresden, Germany

4 Hasso Plattner Institute, University of Potsdam, Potsdam, Germany

5 Dept. of Anesthesia, Dalhousie University, Halifax, Canada

Introduction: The recovery of patients after general anaesthesia (GA) is usually evaluated by the scoring of clinical signs $(1,2)$. These scoring systems do not cover the assessment of psychomotor function, which may be compromised after GA and could lead to serious complications (2). The measurement of 4-choice reaction time (4CRT) is considered an established method to evaluate the psychomotor changes due to sedative medication (3) and was recently validated as the most convenient test to assess the recovery of psychomotor function in patients after GA (4). The aim of this study was to investigate psychomotor recovery after GA in patients with and without anxiolytic premedication in a relationship with conventional discharge scores.

Methods: Local ethics committee approved this investigation. 102 patients scheduled to ambulatory gynaecological surgery under standardized GA (duration<40 minutes) signed the informed consent form and were randomly assigned to receive $0.1 \mathrm{mg} / \mathrm{kg}$ oral midazolam (M group) or no ( $\mathrm{N}$ group) for treatment of preoperative anxiety before surgery. Reaction times using the smartphone App "4CRT", designed for this investigation (5), the grade of sedation (Richmond Agitation-Sedation Scale), discharge ability using Postanaesthesia Discharge Scoring System (PADSS), and hemodynamic parameters were recorded at baseline and at 6 time-points during the investigation and compared between the study groups. In another 22 female patients, who were recruited according to eligibility criteria and all received midazolam, the plasma concentration of this benzodiazepine was measured in addition to other outcome measures and its relationship with 4CRT was analyzed.

Results: Complete data from 94 randomized patients were available for evaluation. The $\mathrm{M}$ group showed prolonged reaction times compared to the $\mathrm{N}$ group 15 minutes following midazolam administration before surgery as well as at time-points 60 and 90 minutes following surgery $(P<0.05$; Figure 1). The patients from both groups met the discharge criteria 60 minutes after surgery. The reaction times of the $\mathrm{M}$ group returned to baseline 90 minutes after surgery. Reaction times of the $\mathrm{N}$ group returned to baseline 45 minutes after surgery but only 23 of the 45 patients in the $\mathrm{N}$ group met the discharge criteria at that time. Plasma midazolam concentration of 22 patients was correlated with 4CRT only at time-point 15 minutes following benzodiazepine administration $(r=0.57 ; P=0.006)$.

Conclusion: After short-term general anaesthesia, the psychomotor function recovers faster than the ability to discharge according to PADSS. Premedication with midazolam prolongs the recovery of psychomotor function without delaying the clinical ability to discharge. The recovery of psychomotor function in patients after long (> 60 minutes) general anaesthesia should be controlled using 4CRT in the future. 


\section{REFERENCES:}

1. Chung et al. A post-anesthetic discharge scoring system for home readiness after ambulatory surgery. J Clin Anesth 1995;7:500-6.

2. Marshall \& Chung. Discharge criteria and complications after ambulatory surgery. Anesth Analg 1999; 88:508-17.

3. Hendry et al. Objective measurement of sedation III: the reaction timer. Brit J Anaesth 1963;35:716-8.

4. Usichenko et al. Computerized 4-choice reaction time test for the measurement of psychomotor recovery after general anesthesia. J Clin Monit Comput. 2019 doi: 10.1007/s10877-019-00355-

5. http://lehmannro.github.io/4crt

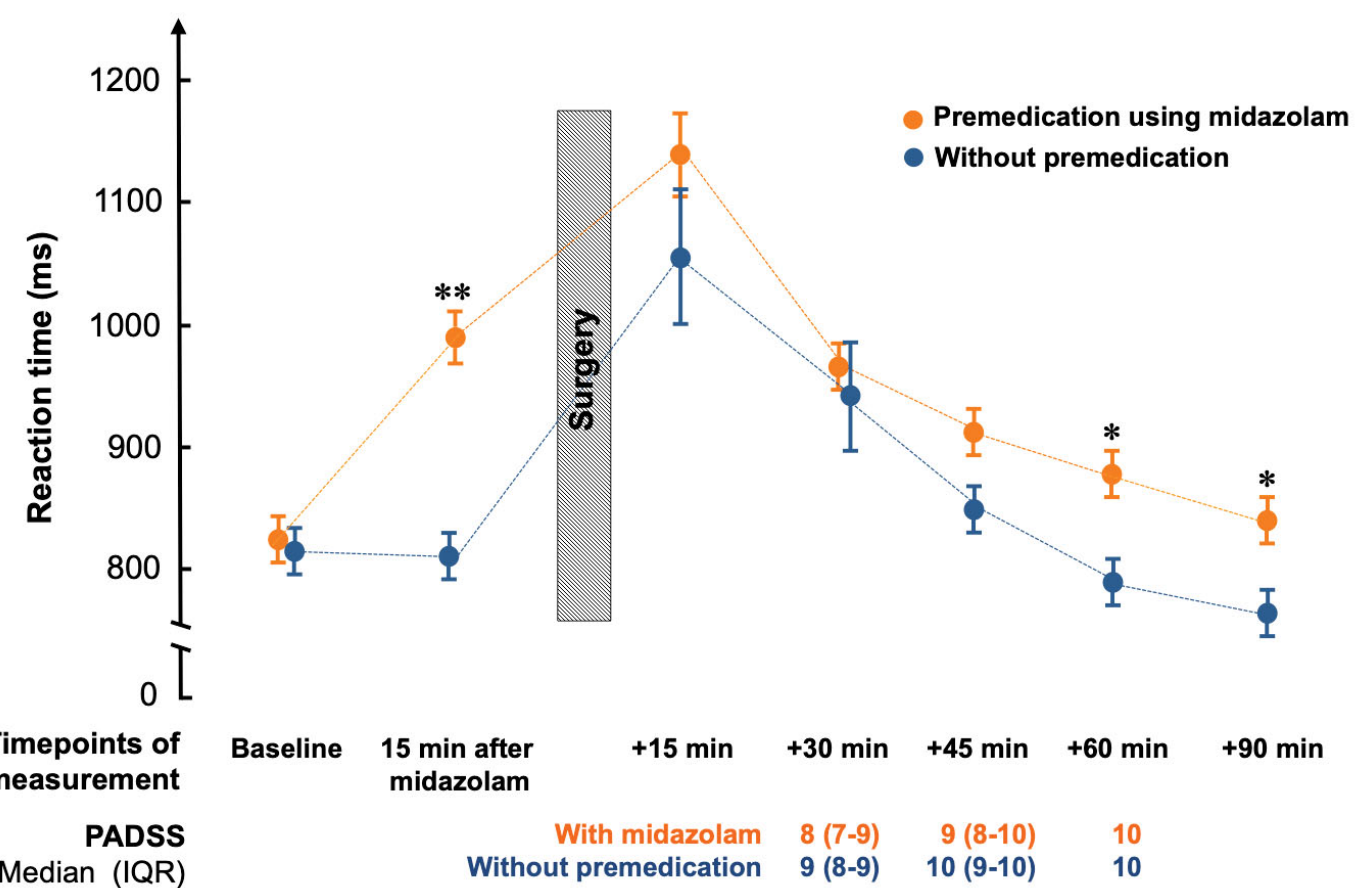

Figure 1: Reaction times measured using the 4-CRT App, given as the mean \pm standard error of mean at various times during the investigation. Comparison between the groups was performed using Student's $t$-test for paired observations with Bonferroni-adjustment for multiple comparisons. $* \mathrm{P}<0.05 ; * * \mathrm{P}<0.0001$. 
Renal Tissue PO2 Measurements Reflect the Degree of Acute Hemodilution and Demonstrate a Negative Impact of Starch Colloid on Renal Oxygenation

$\underline{K y l e ~ C h i n ~}^{1,2}$; Jessica Abrahamson ${ }^{1,2}$; Melina P. Cazorla-Bak ${ }^{1,2}$; Jason T. Maynes ${ }^{3}$; Richard E. Gilbert $^{2,4,5}$; Kim A. Connelly ${ }^{2,4,6}$; Andrew J. Baker ${ }^{1,4,7}$; C. David Mazer ${ }^{1,2,4}$; Gregory M.T. Hare ${ }^{1,2,4}$

1 Department of Anesthesia, St. Michael's Hospital, University of Toronto, Toronto, Ontario, Canada

2 Department of Physiology, University of Toronto, Toronto, Ontario, Canada

3 Department of Anesthesia and Pain Medicine, Hospital for Sick Children, University of Toronto, Toronto, Ontario, Canada

4 Keenan Research Centre for Biomedical Science in the Li Ka Shing Knowledge Institute, St. Michael's Hospital, Toronto, Ontario, Canada

5 Department of Medicine, Division of Endocrinology, St. Michael's Hospital, University of Toronto, Toronto, Ontario, Canada

6 Department of Medicine, Division of Cardiology, St. Michael's Hospital, University of Toronto, Toronto, Ontario, Canada

7 Institute of Medical Science, University of Toronto, Toronto, Ontario, Canada

Introduction: The role of starch colloid solutions in optimal fluid resuscitation remains controversial. Recent clinical trials have demonstrated the potential for starch mediated renal toxicity in critical care and surgical settings $(1,2)$. We assessed the impact of colloid (starch and albumin) and crystalloid (saline) hemodilution protocols in terms of their impact on cardiac output (CO) and renal tissue oxygen tension $\left(\mathrm{P}_{\mathrm{kt}} \mathrm{O}_{2}\right)$. We hypothesized that hemodilution with starch colloid would negatively impact $\mathrm{P}_{\mathrm{kt}} \mathrm{O}_{2}$ relative to other solutions following acute hemodilution.

Methods: Ethics approval was obtained from the local REB, male Sprague Dawley rats $(n=77$, $484 \pm 64 \mathrm{~g}$ ) were randomly assigned to undergo hemodilution with saline (40\% estimated blood volume, $3: 1$ vol:vol), $6 \%$ hydroxyethyl starch (30 or $40 \%$ EBV, $1: 1), 5 \%$ albumin $(40 \% \mathrm{EBV}, 1: 1)$ or time-based control. Heart rate, MAP and rectal temperature were monitored continuously. Arterial blood gases, cooximetry data and echocardiograms were performed at baseline, 30 and 60 minutes post-hemodilution. $\mathrm{P}_{\mathrm{kt}} \mathrm{O}_{2}$ was measured continuously on the left kidney by a phosphorescence quenching method using Oxyphor PdG4 as an oxygen probe. Kidneys were harvested after 60 minutes post-hemodilution determine RNA levels (qPCR) for erythropoietin (EPO), and other hypoxia-induced molecules. Data was tested for normality and analysis was performed by ANOVA or ANCOVA. ( $p<0.05$ was taken to be significant)

Results: All measurements were comparable between groups at baseline. After hemodilution, the hemoglobin concentration $(\mathrm{Hb})$ decreased in all treatment groups; reaching nadir values near $70 \mathrm{~g} / \mathrm{L}$ (40\% albumin and starch) and $90 \mathrm{~g} / \mathrm{L}$ (40\% saline or $30 \%$ starch) respectively ( $p<0.005$ for both). Mean arterial pressure (MAP) decreased slightly after hemodilution, but without significant difference between groups. Arterial $\mathrm{pO}_{2}$ was not significantly different between groups. Changes in cardiac output were transient and values were comparable between groups after hemodilution. The final $\mathrm{P}_{\mathrm{kt}} \mathrm{O}_{2}$ was lowest after $40 \%$ hemodilution with starch $(34.1 \pm 6.6 \mathrm{mmHg})$ and significantly different relative to $40 \%$ hemodilution with saline 
(46.9 $\pm 5.9 \mathrm{mmHg} ; \mathrm{p}<0.02)$. A significant linear relationship between changes in $\mathrm{P}_{\mathrm{kt}} \mathrm{O}_{2}$ relative to changes in $\mathrm{C}_{a} \mathrm{O}_{2}$ was observed. The slope of the relationship was significantly steeper for starch hemodilution relative to albumin and saline ( 0.333 versus 0.222 respectively, $p<0.008)$. Renal tissue mRNA levels for EPO were increased substantially after starch and saline hemodilution $(p<0.05)$.

Conclusion: Kidney tissue oxygen tension decreased in proportion with the drop in $\mathrm{C}_{\mathrm{a}} \mathrm{O}_{2}$ following hemodilution regardless of the diluent. Hemodilution with starch resulted in a more pronounced reduction in $\mathrm{P}_{\mathrm{kt}} \mathrm{O}_{2}$ and an increase in EPO mRNA levels, indicative of impaired microvascular oxygen delivery and accentuated tissue hypoxia. The more favorable hemodynamic and $\mathrm{P}_{\mathrm{kt}} \mathrm{O}_{2}$ responses following hemodilution with albumin warrant further investigation.

\section{REFERENCES:}

1. Futier E, Garot M, Godet T, Biais M, Verzilli D, Ouattara A, Huet O, Lescot T, Lebuffe G, Dewitte A, Cadic A, Restoux A, Asehnoune K, Paugam-Burtz C, Cuvillon P, Faucher M, Vaisse C, El Amine Y, Beloeil H, Leone M, Noll E, Piriou V, Lasocki S, Bazin J-E, Pereira B, Jaber S, and Group ftFT. Effect of Hydroxyethyl Starch vs Saline for Volume Replacement Therapy on Death or Postoperative Complications Among High-Risk Patients Undergoing Major Abdominal Surgery: The FLASH Randomized Clinical Trial. Jama 323: 225-236, 2020.

2. Zarychanski R, Abou-Setta AM, Turgeon AF, Houston BL, Mclntyre L, Marshall JC, and Fergusson DA. Association of hydroxyethyl starch administration with mortality and acute kidney injury in critically ill patients requiring volume resuscitation: a systematic review and meta-analysis. Jama 